Portland State University

PDXScholar

$6-4-2020$

\title{
The Role of Strategic Governance in Reducing Infant Mortality Under Crisis Conditions
}

Lynn Christine Finley

Portland State University

Follow this and additional works at: https://pdxscholar.library.pdx.edu/open_access_etds

Part of the Public Health Commons, and the Public Policy Commons Let us know how access to this document benefits you.

Recommended Citation

Finley, Lynn Christine, "The Role of Strategic Governance in Reducing Infant Mortality Under Crisis Conditions" (2020). Dissertations and Theses. Paper 5475.

https://doi.org/10.15760/etd.7347

This Dissertation is brought to you for free and open access. It has been accepted for inclusion in Dissertations and Theses by an authorized administrator of PDXScholar. Please contact us if we can make this document more accessible: pdxscholar@pdx.edu. 
The Role of Strategic Governance in Reducing Infant Mortality Under Crisis Conditions

by
Lynn Christine Finley

A dissertation submitted in partial fulfillment of the requirements for the degree of

\author{
Doctor of Philosophy \\ in \\ Public Affairs and Policy
}

Dissertation Committee:

Bruce Gilley, Chair

Lindsay Benstead

Birol Yesilada

John Gallup

Portland State University

2020 
(C) 2020 Lynn Christine Finley 


\begin{abstract}
The infant mortality rate (IMR) in some developing countries has decreased faster than the global average even though these countries lack strong economic growth, good governance, and democracy (often acknowledged precursors to improved health outcomes). What accounts for the improvement of the IMR in the absence of these traditional pathways to health gains? Some scholars suggest that the concept of "strategic governance" might help direct attention to intermediary factors that reduce neonatal deaths in countries that experience crisis conditions. The main objective of this dissertation is to investigate the set of governance practices that have reduced IMR in two such cases. In particular, what "strategic governance" practices, if any, can be identified as contributing to the IMR decline in such cases? Using case studies to explore the significant reduction in IMR in Republic of Congo (ROC) and Republic of Yemen (ROY), this dissertation finds that micro-strategic governance interventions such as decentralization, international partnerships, funding, performance-based funding and cultural change can mitigate the macrostructural crisis that threatens IMR in developing countries.
\end{abstract}




\section{Acknowledgments}

I cannot begin to express my deepest gratitude to Dr. Bruce Gilley for his unparalleled support and invaluable contribution to this dissertation. I am also indebted to my committee members: Dr. Birol Yesilada, Dr. Lindsay Benstead, Dr. John Gallup, and Dr. Shawn Smallman for their insightful suggestions and time. In addition, I had the great pleasure to work with the following: Dr. David Kinsella, Dr. Christopher Shortell, Dr. Meoldy Valdini, Dr. Craig Shinn, Dr. Jack Corbett, and Dr. Phillip J. Cooper during my enrollment at Portland State University. Thanks also go to Dr. Laurie Drapela, Dr. M. Jahi Chappell, Dr. Paul Thiers, Dr. Mark Stephan, and Dr. Dana Baker for their guidance during my studies at Washington State University, Vancouver. 


\section{Table of Contents}

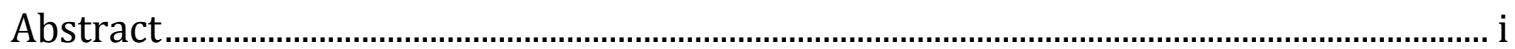

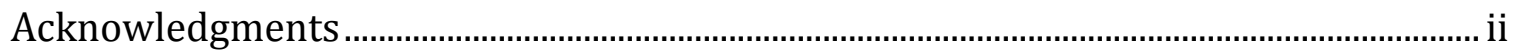

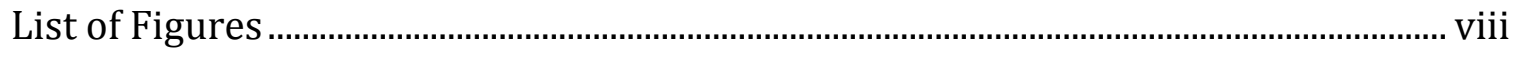

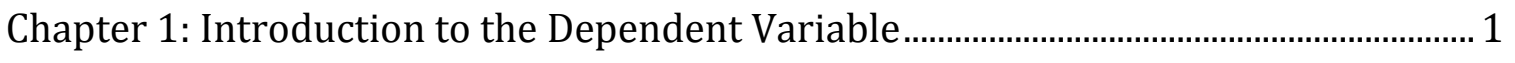

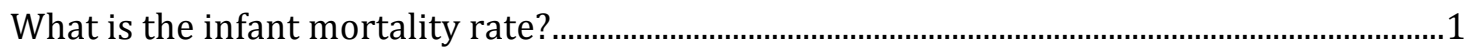

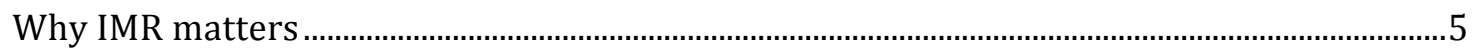

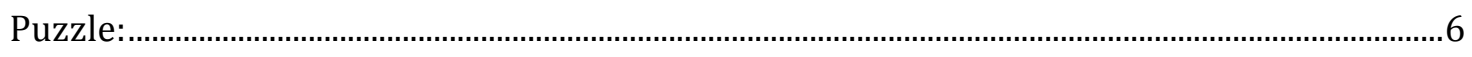

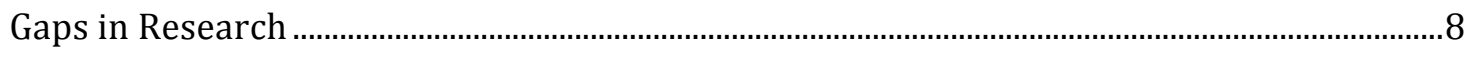

Chapter 2: Literature Review on Causes of IMR Improvement...................................... 10

The direct or proximate physiological mechanisms of infant mortality .................................13

The indirect health mechanisms that alleviate the physiological mechanisms of infant

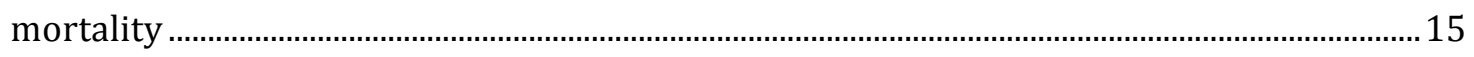

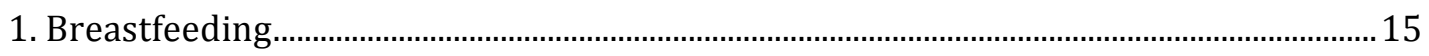

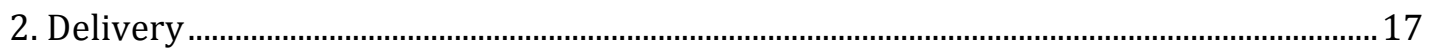

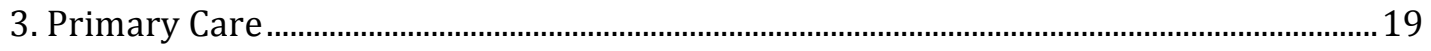

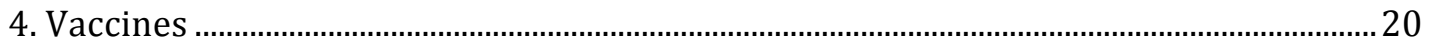

The indirect social and policy mechanisms that alleviate the physiological mechanisms of

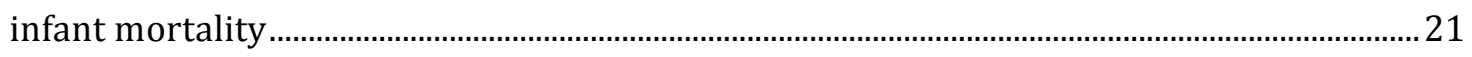

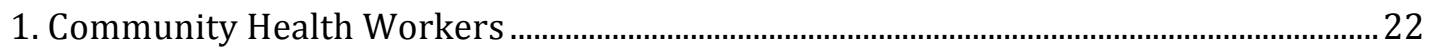

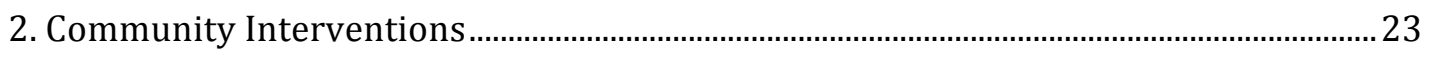

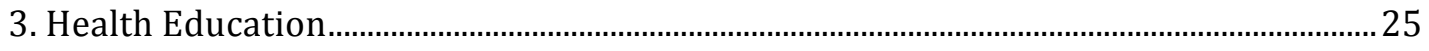




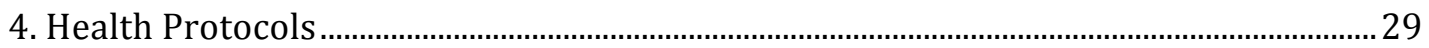

5. Preventative Actions Against Risk Factors .............................................................................. 31

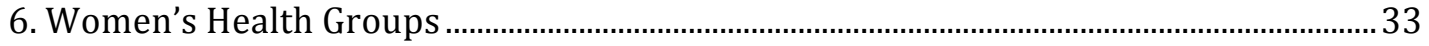

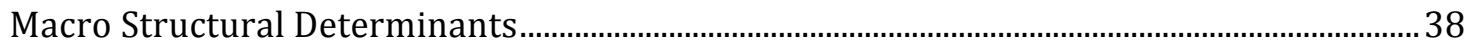

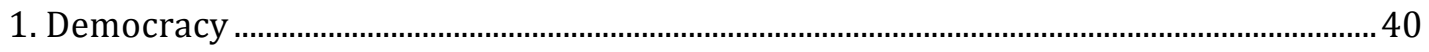

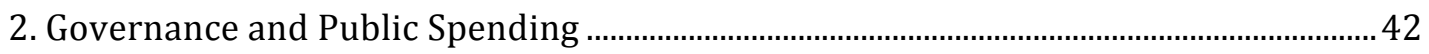

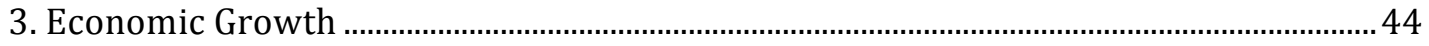

Macro-negative (structural pessimism) vs. Micro-positive (agency optimism) ....................47

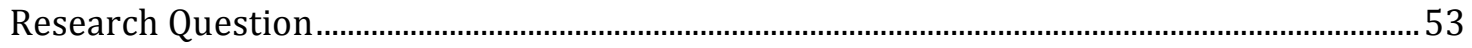

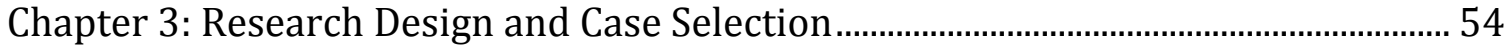

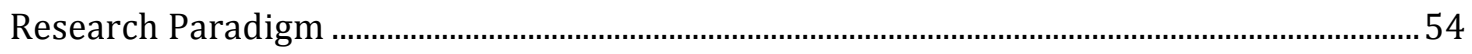

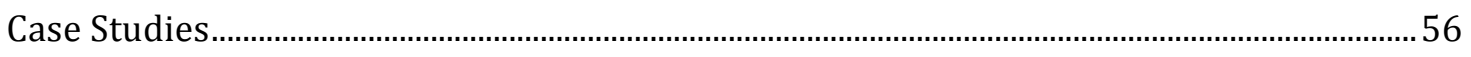

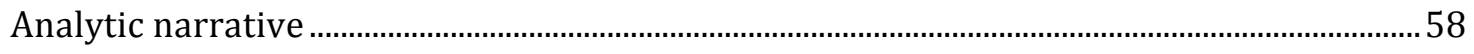

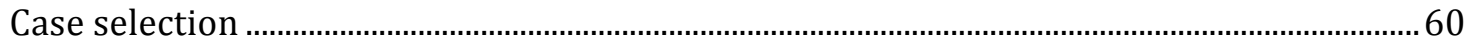

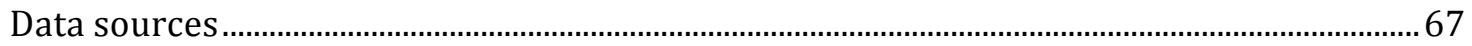

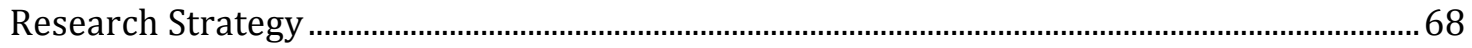

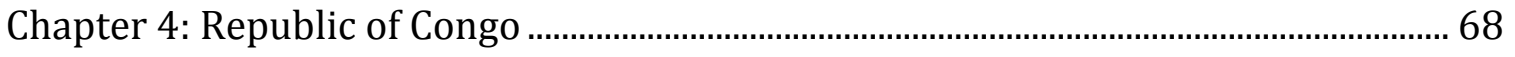

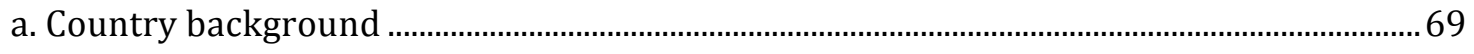

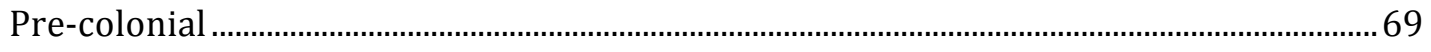

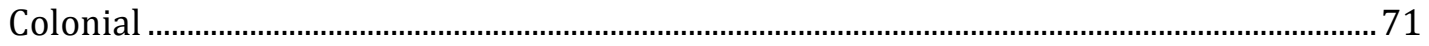

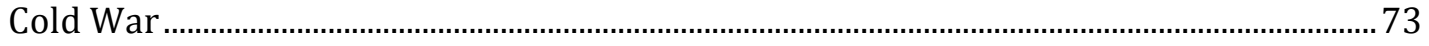

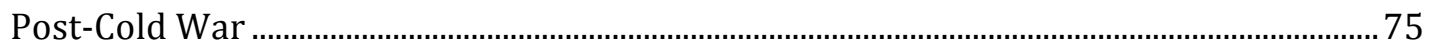

b. Description of macro-structural weakness ........................................................................... 78 


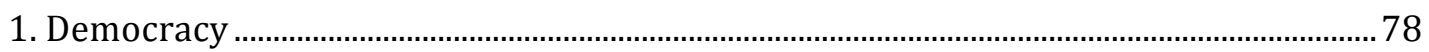

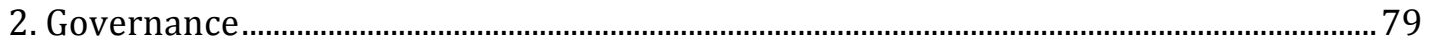

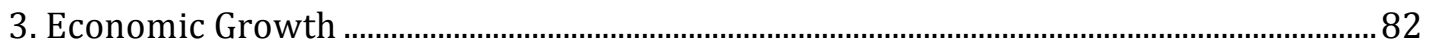

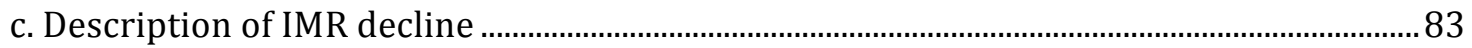

d. Description of indirect mechanisms that alleviate the proximate causes of infant

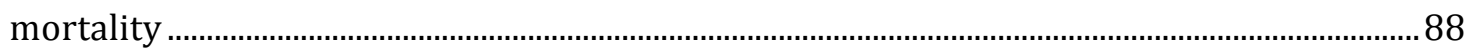

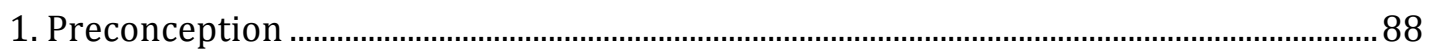

2. Antenatal Care/Prenatal Care ............................................................................................ 92

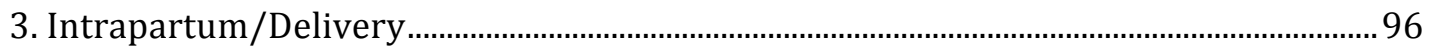

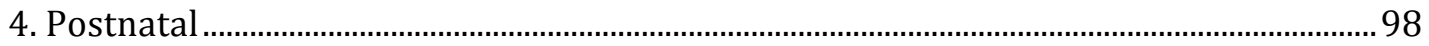

e. Case study process tracing and analytic narrative development........................................ 103

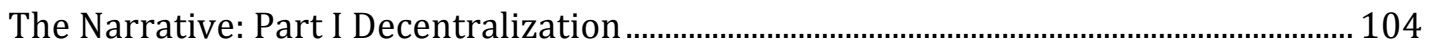

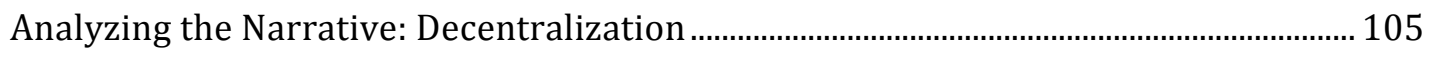

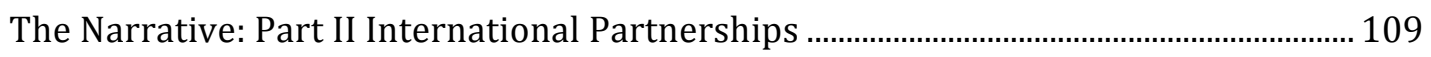

Analyzing the Narrative: International Partnerships ....................................................... 110

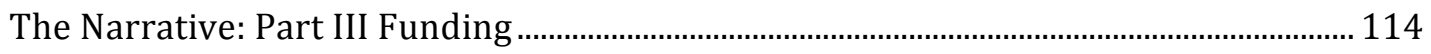

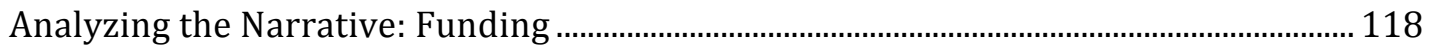

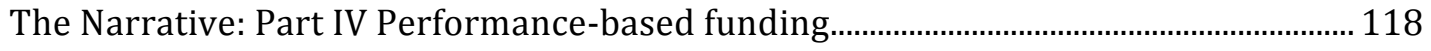

Analyzing the Narrative: Performance-based funding............................................................. 120

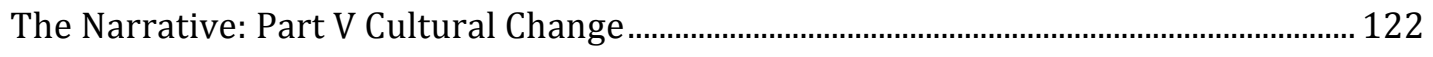

Analyzing the Narrative: Cultural change........................................................................... 124

f. Initial statement of "strategic governance" findings........................................................ 127

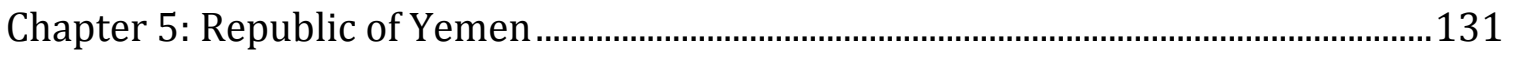




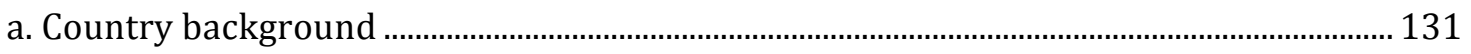

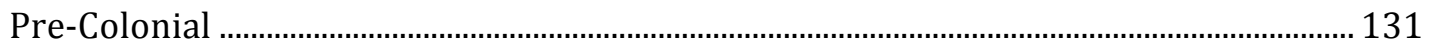

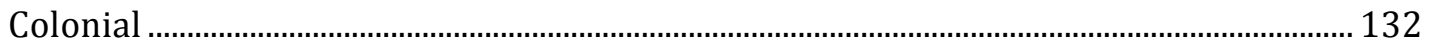

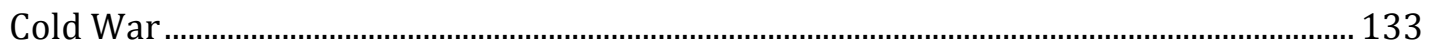

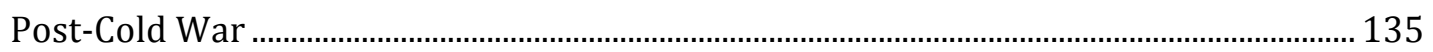

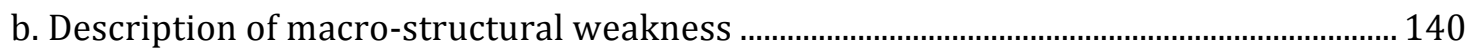

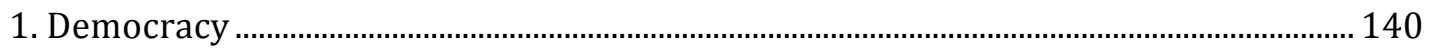

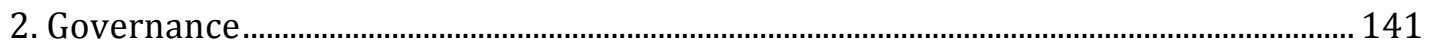

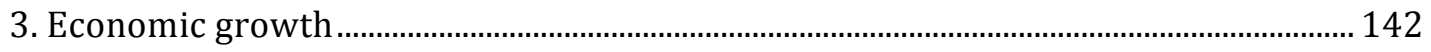

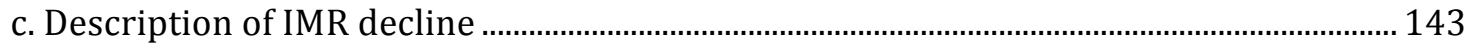

d. Description of indirect mechanisms that alleviate the proximate physiological causes of

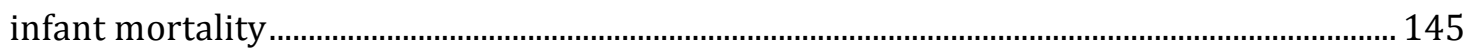

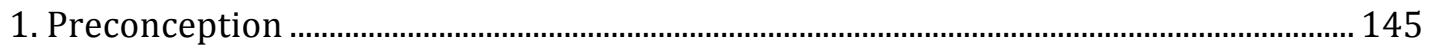

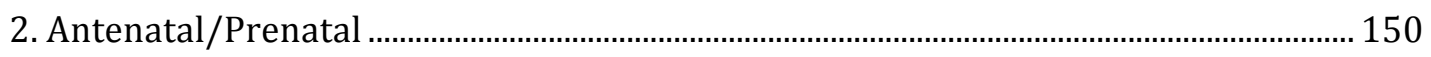

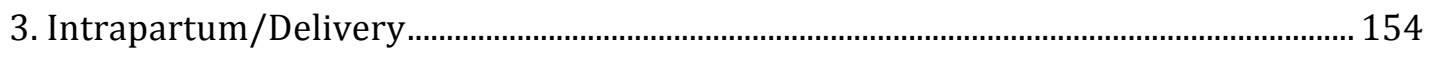

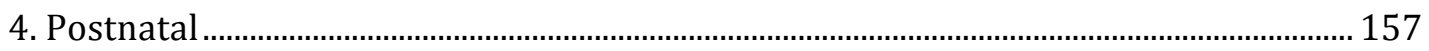

e. Case study analytic narrative and analyzing the narrative .................................................. 157

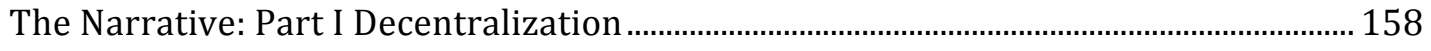

Analyzing the Narrative: Decentralization ……….................................................................. 162

The Narrative: Part II International Partnerships …………............................................... 165

Analyzing the Narrative: International Partnerships ......................................................... 168

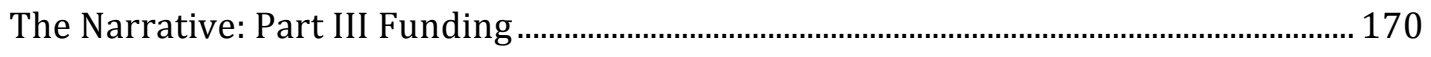

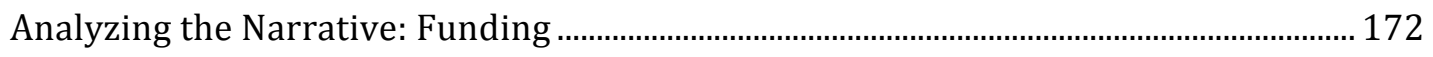

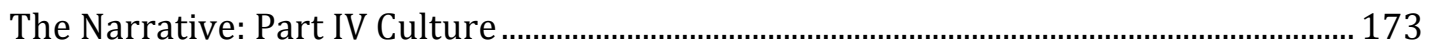


Analyzing the Narrative: Culture

f. Initial statement of "strategic governance" findings.......................................................... 178

Chapter 6: Research Findings on Strategic Governance...................................................181

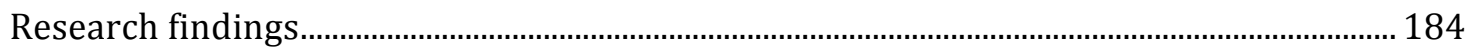

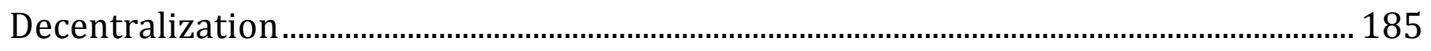

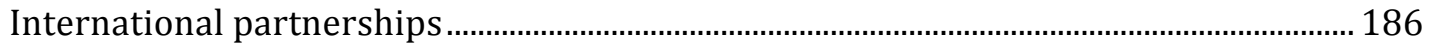

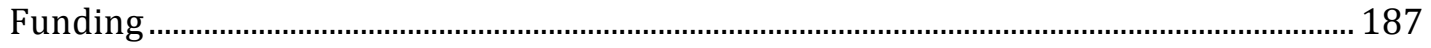

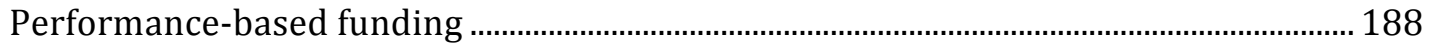

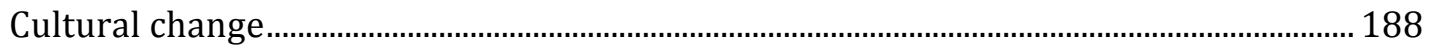

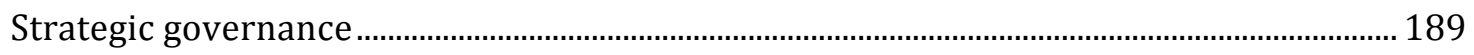

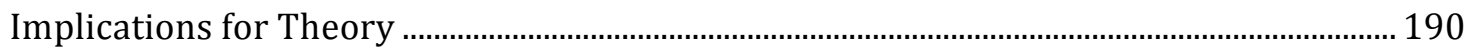

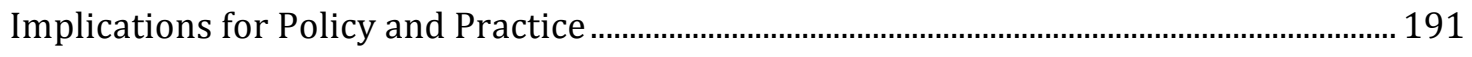

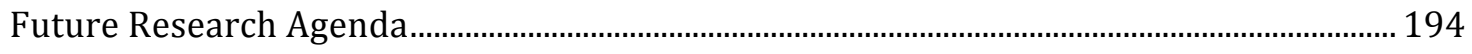

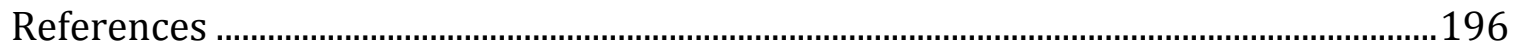

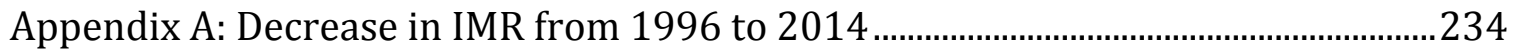

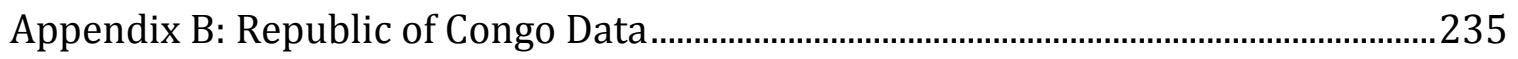

Appendix C: Select Survey Data for Republic of Congo .....................................................236

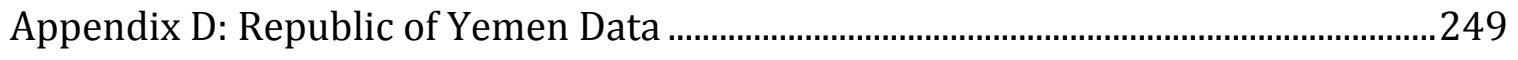

Appendix E: Select Survey Data for Republic of Yemen..................................................250 


\section{List of Figures}

Figure 1: Three indicator levels of IMR- one direct, two indirect.................................... 13

Figure 2: Chronology of potential care phases in a woman's life .................................... 36

Figure 3: Intersection of the two indirect mechanisms that alleviate infant mortality with the chronology of potential care phases during a woman's life..................... 38

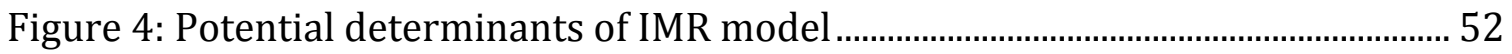

Figure 5: 106 developing countries as measured by average governance scores and

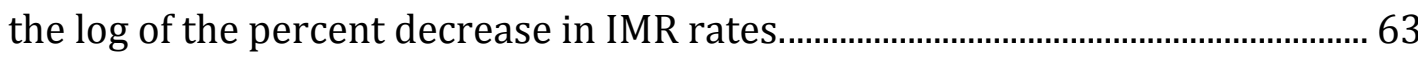

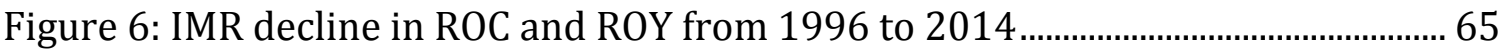

Figure 7: Decrease in family planning messages through different media over time

Figure 8: Strategic governance at preconception phase (ROC)....................................... 92

Figure 9: Received 2+ tetanus injections and overall gains in ANC provider between

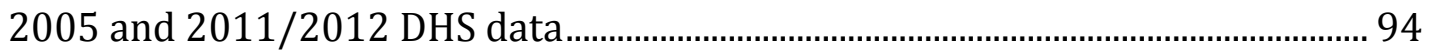

Figure 10: Strategic governance at antenatal/prenatal phase (ROC)........................... 96

Figure 11: Delivery location and type of assistance at delivery between 2005 and $2011 / 2012$ 97

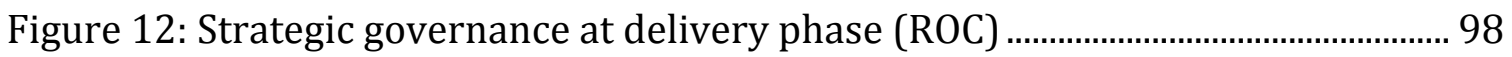

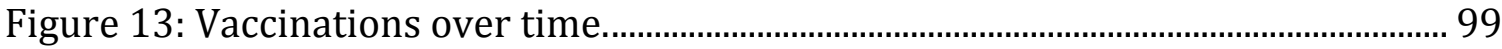

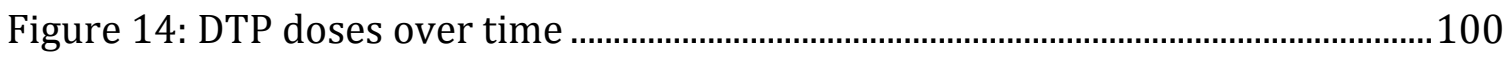

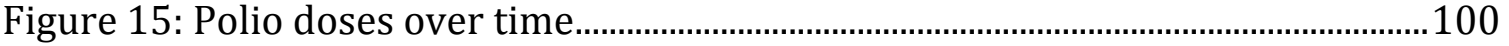


Figure 16: Knowledge of HIV transmission by breastfeeding and knowledge of special drugs by location between 2005 and 2011/2012 ….................................102

Figure 17: Strategic governance at postnatal phase (ROC)............................................103

Figure 18: Awareness of family planning ........................................................................147

Figure 19: Median age of first birth by location over time ..............................................148

Figure 20: Strategic governance at preconception phase (ROY) ..................................150

Figure 21: Number of antenatal care (ANC) visits over time ........................................151

Figure 22: Strategic governance at antenatal/prenatal phase (ROY)............................154

Figure 23: Location of delivery over time .....................................................................156

Figure 24: Level of assistance during delivery over time ……………………………....156

Figure 25: Strategic governance at delivery phase (ROY) .............................................157

Figure 26: The 12 components of health sector reform strategy ……………..............160

Figure 27: Priority district level health care diseases, Ministry of Public Health 2000

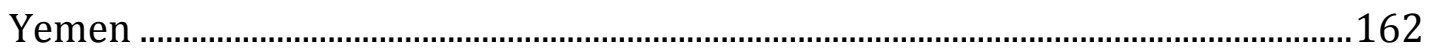


Chapter 1: Introduction to the Dependent Variable

What is the infant mortality rate?

Human development, Sen explains, is about having the freedom to make the most of life through opportunity ${ }^{1}$. Opportunity is frequently expressed or defined through economics but it is also defined by one's ability to partake in "the social, political, and economic life"2. The Human Development Index (HDI) defines levels of similar concepts by a composite measure of life expectancy at birth, expected years of schooling, mean years of schooling, and GNI per capita to demonstrate the global ranking of nations ${ }^{3}$. Another way to define human development is to look at the infant mortality rate (IMR) as it too is a good proxy for the overall development of a country and is useful as a "cross-national and historical measure" according to Gerring, Thacker, and Alfaro. ${ }^{4}$ The IMR is an indicator of "population health" and is considered "particularly sensitive to general structural factors, like socio-economic development and basic living conditions"5. This paper uses the IMR as a definition of development in countries based on Gerring et al.

${ }^{1}$ Amartya Sen, Development as Freedom, 1st ed. (New York: Knopf, 1999), 3.

2 Sen, 4.

3 "Human Development Index (HDI) | Human Development Reports," accessed September 27, 2019, http://hdr.undp.org/en/content/human-development-index-hdi.

4 John Gerring, Strom Thacker, and Rodrigo Alfaro, "Democracy and Human Development," The Journal of Politics 74, no. 1 (January 2012): 4-5.

5 Benn KD Sartorius and Kurt Sartorius, "Global Infant Mortality Trends and Attributable Determinants-an Ecological Study Using Data from 192 Countries for the Period 1990-2011," Population Health Metrics 12, no. 1 (2014): 1. 
The IMR of a country is defined as the number of infant deaths prior to the age of one per 1,000 live births $s^{6}$. The infant mortality rate is measured from birth to age one, but other common measurements include perinatal mortality (from 154 days of gestation to 7 complete days after birth ${ }^{7}$ ), the time between birth and the first month of life (neonatal mortality rate), and after the neonatal period to age 1 (post neonatal mortality rate) ${ }^{8}$ and children under- 5 years of age (under-5 mortality rate) ${ }^{9}$. Many child deaths occur in the first days of life. Neonatal death is so prevalent in some regions that it is not even considered a "problem" because it is a common occurrence ${ }^{10}$. More people die in the neonatal period than any other time in life of the same length ${ }^{11}$. Globally, in 2003, 3.9 million out of the 10.8 million under-5 child deaths occurred in the neonatal period (the first 28 days of life) ${ }^{12}$. By 2010, there were 7.7 million under-5 deaths of which 3.1 million were neonatal, 2.3

\footnotetext{
6 Gerring, Strom Thacker, and Alfaro, “Democracy and Human Development," 4.

${ }^{7}$ Hasan S Merali et al., "Audit-Identified Avoidable Factors in Maternal and Perinatal Deaths in Low Resource Settings: A Systematic Review," BMC Pregnancy and Childbirth 14, no. 1 (December 2014): 2, https://doi.org/10.1186/1471-2393-14-280.

8 Julie Knoll Rajaratnam et al., "Neonatal, Postneonatal, Childhood, and under-5 Mortality for 187 Countries, 1970-2010: A Systematic Analysis of Progress towards Millennium Development Goal 4," The Lancet 375, no. 9730 (2010): 1993.

${ }^{9}$ Robert E Black, Saul S Morris, and Jennifer Bryce, "Where and Why Are 10 Million Children Dying Every Year?," The Lancet 361, no. 9376 (June 28, 2003): 2226, https://doi.org/10.1016/S01406736(03)13779-8.

10 World Health Organization, "Neonatal and Perinatal Mortality: Country, Regional and Global Estimates," 2006, 2.

11 William Moss et al., "Research Priorities for the Reduction of Perinatal and Neonatal Morbidity and Mortality in Developing Country Communities," Journal of Perinatology 22, no. 6 (September 2002): 452, https://doi.org/10.1038/sj.jp.7210743.

12 Black, Morris, and Bryce, “Where and Why Are 10 Million Children Dying Every Year?," 2229.
} 
million were post neonatal, and 2.3 million were post neonatal to 4 years of age ${ }^{13}$. The greatest proportion of deaths occurred in the neonatal period. Out of all neonatal deaths globally, $98 \%$ of them occur in the developing world, "most often at home, outside the formal health care system, and largely due to infections ${ }^{14}$. SubSaharan Africa (SSA) (from 1990-2011) had the highest IMR globally (75 infant deaths per 1,000 live births), contrasted with Europe, during the same time frame, with an IMR of $11^{15}$. Neonatal mortality is largely the result of infections, intrapartum conditions, and preterm birth complications ${ }^{1617}$. IMR determinants can be ranked by "proximal (e.g., infectious), intermediate (e.g., water and sanitation), and distal (e.g., socio-economic status, education) factors"181920. Annually, improving the quality of care for mothers and newborns could prevent approximately 1.3

\footnotetext{
13 Rajaratnam et al., "Neonatal, Postneonatal, Childhood, and under-5 Mortality for 187 Countries, 1970-2010," 1988.

14 Moss et al., "Research Priorities for the Reduction of Perinatal and Neonatal Morbidity and Mortality in Developing Country Communities," 484.

15 Sartorius and Sartorius, "Global Infant Mortality Trends and Attributable Determinants-an Ecological Study Using Data from 192 Countries for the Period 1990-2011,” 1.

16 Joy E Lawn et al., "Every Newborn: Progress, Priorities, and Potential beyond Survival," The Lancet 384, no. 9938 (July 2014): 189, https://doi.org/10.1016/S0140-6736(14)60496-7.

17 Moss et al., "Research Priorities for the Reduction of Perinatal and Neonatal Morbidity and Mortality in Developing Country Communities," 484.

18 Sartorius and Sartorius, "Global Infant Mortality Trends and Attributable Determinants-an Ecological Study Using Data from 192 Countries for the Period 1990-2011,” 2.

19 W. Henry Mosley and Lincoln C. Chen, "An Analytical Framework for the Study of Child Survival in Developing Countries," Population and Development Review 10 (1984): 25, https://doi.org/10.2307/2807954.

20 Carl Otto Schell et al., "Socioeconomic Determinants of Infant Mortality: A Worldwide Study of 152 Low-, Middle-, and High-Income Countries," Scandinavian Journal of Public Health 35, no. 3 (May 2007): 290, https://doi.org/10.1080/14034940600979171.
} 
million neonatal deaths ${ }^{21}$. Neonatal deaths are "mainly caused by preventable and treatable conditions"22. A significant portion of perinatal and neonatal deaths could be prevented by low-cost and relatively straightforward improvements in the continuum of care for mother and baby ${ }^{23}$.

Over the past century, IMR declined except for a period at the turn of the century in sub-Saharan Africa due to HIV24 but the overall trend continued downward.

Reduction in child mortality, which is partly driven by decreasing infant mortality rates, was also one of the eight United Nation's Millennium Development Goals that brought the "limelight" 25 to focus on infant survival. The under-5 mortality rate decreased 49\% between 1990 and 201326. In 2013, there were 2.8 million neonatal deaths worldwide, which comprised about $44 \%$ of the under- 5 deaths ${ }^{27}$. Countries with low infant mortality rates generally have better development than countries with high infant mortality rates.

21 Zulfiqar A Bhutta et al., "Can Available Interventions End Preventable Deaths in Mothers, Newborn Babies, and Stillbirths, and at What Cost?," The Lancet 384, no. 9940 (July 2014): 347, https://doi.org/10.1016/S0140-6736(14)60792-3.

22 Organization, "Neonatal and Perinatal Mortality," 25.

23 Moss et al., "Research Priorities for the Reduction of Perinatal and Neonatal Morbidity and Mortality in Developing Country Communities," 485.

24 Sartorius and Sartorius, "Global Infant Mortality Trends and Attributable Determinants-an Ecological Study Using Data from 192 Countries for the Period 1990-2011," 1.

25 Lawn et al., "Every Newborn,” 189.

26 “New Data Show Child Mortality Rates Falling Faster than Ever," UNICEF, accessed June 5, 2019, https://www.unicef.org/media/media_75892.html.

27 “New Data Show Child Mortality Rates Falling Faster than Ever." 
Why IMR matters

The IMR of a country matters because it is inherently good to improve the human condition, and it is a close correlate of development within countries. As

development improves, human potential improves and the life choices for countries and its citizens (and their place in in the economy) are much improved ${ }^{28}$.

Development is, of course, more than just economic; human development is frequently the goal of economic development because of "what people can do and be"29. As infant mortality drops there is a fairly well established trend that suggests countries are more politically stable, just as "the countries with the highest NMRs tend to congregate in the lower portion of the political stability scale" ${ }^{30}$. For example, Wise and Darmstadt (2015) demonstrate Somalia was at the lowest end of the political stability scale based off of data from the Worldwide Governance Indicators for political stability with a score of 0 , while Lesotho received a score of 58. The authors reveal there is an "inverse relationship between political stability and neonatal mortality rate" but they also recognize that there can be some variation $^{31}$. Development is typically associated with stability, democratic regimes, prosperity, peace, and solid governance practices, i.e., practices that promote

\footnotetext{
28 Lawn et al., "Every Newborn,” 189.

29 Sudhir Anand and Martin Ravallion, "Human Development in Poor Countries: On the Role of Private Incomes and Public Services," Journal of Economic Perspectives 7, no. 1 (March 1993): 133, https://doi.org/10.1257/jep.7.1.133.

30 Paul H. Wise and Gary L. Darmstadt, "Strategic Governance: Addressing Neonatal Mortality in Situations of Political Instability and Weak Governance," Seminars in Perinatology, Global Perinatal Medicine, 39, no. 5 (August 2015): 388, https://doi.org/10.1053/j.semperi.2015.06.008.

31 Wise and Darmstadt, 388.
} 
wellbeing and opportunity for human agency. Still, some countries without these conditions have experienced rapid declines in IMR.

Puzzle:

For example, how did North Korea reduce IMR by 62\% between 1996 and 2014 despite an authoritarian government? Or how did Rwanda, with an IMR of 120.3 infant deaths per 1,000 in 1996, achieve 32.7 infant deaths per 1,000 in 2014 (a $73 \%$ decline in the IMR)?

This dissertation will investigate how some countries reduce IMR despite dysfunction and structural hurdles. The IMR will be measured in cross-national studies to demonstrate which countries are doing well, average, or poorly to mitigate preventable deaths ${ }^{32}$ in the context of low levels of economic growth, governance, and democracy. The "exceptional" countries will then be used as the basis of inductive theorizing to explore how the IMR was improved, and better understand the potential of "strategic governance" to improve health outcomes. The intention is to use these lessons to apply to development more broadly.

Globally the annual IMR decline between 1990 and 2010 was " $2.1 \%$ for neonatal mortality, $2.3 \%$ for post neonatal mortality, and $2.2 \%$ for childhood mortality"33. From 2000 to 2010 research reveals that IMR declines increased "by more than $1 \%$

\footnotetext{
32 Gerring, Strom Thacker, and Alfaro, "Democracy and Human Development.”

33 Rajaratnam et al., "Neonatal, Postneonatal, Childhood, and under-5 Mortality for 187 Countries, 1970-2010," 1988.
} 
in Angola, Botswana, Cameroon, Congo, Democratic Republic of the Congo, Kenya, Lesotho, Liberia, Rwanda, Senegal, Sierra Leone, Swaziland, and The Gambia"34. How then have Africa and other countries improved their IMR when national level structures are functioning poorly? Wise and Darmstadt (2015) propose "strategic governance" 35 to potentially reduce neonatal deaths in countries that experience political instability and poor governance. They describe strategic governance as the "minimal conditions of political stability and governance required for health service implementation"36 in contrast to more ambitious forms of governance, i.e. good governance. However, it is unknown what those "minimal conditions" are. Wise and Darmstadt suggest specific life-saving health implementation can be facilitated in countries to improve health outcomes. Strategic governance is offered as an alternative to the growing list of good governance ${ }^{37}$ requirements. For many countries, it is unrealistic (if not impossible) to achieve all the necessary conditions of good governance. In those cases, opportunity can be found in politically unstable, poorly governed, and low resource environments to achieve improvement in the infant mortality rate. There may exist a set of conditions, variables and interactions between them, such as proven technology, local capacity, resource mobilization, the presence of health experts and supportive "local political and governance

\footnotetext{
${ }^{34}$ Rajaratnam et al., 1988.

35 Wise and Darmstadt, "Strategic Governance," 387.

36 Wise and Darmstadt, 387.

37 Merilee S. Grindle, “Good Enough Governance Revisited," Development Policy Review 25, no. 5 (September 1, 2007): 533-74, https://doi.org/10.1111/j.1467-7679.2007.00385.x.
} 
contexts" 38 that decrease the infant mortality rate and improve human development. Perhaps these unknown interactions comprise the basis of strategic governance.

\section{Gaps in Research}

As the word suggests, "strategic" refers to planning and actions aligned with the achievement of long-term outcomes or goals. We might contrast this with "procedural governance" where actions are designed to conform to procedural rules or "policy governance" in which actions are designed to conform to intended policy or program outputs. Thus in the context of governance, Pouw and de Bruijne (2015) discuss strategic governance as: "the effective realignment of predefined strategies with performances and outcomes within a context of interactive governance." ${ }^{39}$ But, the concept of strategic governance as put forth by Wise and Darmstadt (2015) is open for research. Wise and Darmstadt (2015) call for "strategic governance" to improve health outcomes ${ }^{40}$ which refers specifically to health and to broader development goals but they fail to identify what strategic governance entails. Wise and Darmstadt (2015) suggest that in complex conditions strategic governance is a "substrate for the provision of certain public goods" 41 . For example, Nigeria, Haiti, Somalia, and Zimbabwe experienced reductions in particular illnesses because

\footnotetext{
38 Wise and Darmstadt, "Strategic Governance," 391.

${ }^{39}$ Nicky R. M. Pouw and Ad de Bruijne, "Strategic Governance for Inclusive Development," The European Journal of Development Research 27, no. 4 (September 1, 2015): 483, https://doi.org/10.1057/ejdr.2015.53. italics added 40 Wise and Darmstadt, "Strategic Governance."

41 Wise and Darmstadt, 391.
} 
of service provision even in the face of instability. Strategic governance looks at the "minimal conditions of political stability and governance required for health service implementation"42. Deaton (2006) proposes that health improvements in poor countries come from something other than income, health knowledge or technology. However, he too is vague about what this specifically entails but he does detail that social factors impact the delivery of health services ${ }^{43}$. In the following chapter, this dissertation will identify a plausible and specific hypothesis of strategic governance for IMR declines in the context of existing literature. What explains declines in IMR where economic growth, governance, and democratic accountability are weak? In particular, what "strategic governance" practices, if any, can be identified as contributing to the IMR decline in such cases?

\footnotetext{
42 Wise and Darmstadt, 387.

43 Angus Deaton, “Global Patterns of Income and Health: Facts, Interpretations, and Policies” (National Bureau of Economic Research, 2006), 22, http://www.nber.org.proxy.lib.pdx.edu/papers/w12735.
} 


\section{Chapter 2: Literature Review on Causes of IMR Improvement}

Infant mortality is caused by physiological factors ${ }^{44}$. These physiological factors of infant mortality can be indirectly alleviated by health, and social and policy mechanisms. This research found there are generally ten concepts that organize the literature regarding how the indirect mechanisms of infant mortality are improved. Four of the ten concepts focus on indirect health mechanisms (i.e., mechanisms that are nestled closer to the proximate causes of infant mortality), and the other six concepts focus on social and policy mechanisms (i.e., mechanisms that are slightly removed from the proximate causes of infant mortality but that also make a big difference). The indirect health mechanisms identified from the literature that improve IMR includes: 1) breastfeeding, 2) delivery, 3) primary care, and 4) vaccines. The indirect social and/or policy mechanisms identified from the literature that improve IMR include: 1) community health workers, 2) community intervention, 3) health education, 4) health protocols, 5) preventative actions, and 6) women's health groups.

We can see the impact of both the indirect health mechanisms, as well as the indirect social and policy mechanisms on IMR in the example of a young girl, Fati, in rural Ghana. For instance, Fati is fifteen years old, attends school, and participates in after school activities. Community health workers often present information in her after school program about sanitation and nutrition. Fati learns about the necessity

\footnotetext{
${ }^{44}$ James C. Cramer, "Social Factors and Infant Mortality: Identifying High-Risk Groups and Proximate Causes,” Demography 24, no. 3 (August 1987): 299-322.
} 
for certain health protocols like hand cleaning, and she learns about specific vegetables that provide vital nutrition (she shares this information with her parents who are farmers). Some days Fati learns about her rights as a child, how to avoid harm and how child marriage is not legal. The importance of immunizations is emphasized and Fati encourages her parents to take her and her siblings to receive necessary vaccination when health partners organize clinic days in the community. In this example, Fati is learning specific skills that will improve her health and the health of her future children through indirect mechanisms of health education, preventative actions, vaccines, and community health workers. These key indirect mechanisms can potentially alleviate the proximate causes of infant mortality for her future children.

Now, the figure below incorporates some of the key indirect mechanisms that Fati learned about that can ultimately improve IMR, as well as other indirect mechanisms. The figure has at its center a bulls-eye labeled IMR, as that is the central "target" of discussion. Surrounding IMR are three concentric rings. The first ring surrounding IMR represents the proximate physiological causes of infant mortality: i.e., poor nutrition, disease, and low infant weight. This physiological ring is not explored later in the case studies but it does serve to remind us of how infant mortality can be indirectly alleviated by the second and third concentric rings. The second surrounding ring represents indirect "health" interventions that alleviate the proximate causes of infant mortality. For example, breastfeeding and vaccines lessen instances of malnutrition and disease from the physiological ring. The third 
surrounding ring in the figure represents the indirect "social and policy" mechanisms that also alleviate the proximate physiological mechanisms of infant mortality, i.e., through health education. The indirect health mechanism level is represented closest to the physiological ring because it is the first level of indirect mechanisms to impact the proximate causes of death, many of which happen at the time of birth. As such, it is represented closer to the IMR bulls-eye than the second indirect indicator level of social and policy mechanisms. The indirect health indicators also reflect the "last mile" of care a woman receives around the time of birth, a critical time for infant health. Next, we see the indirect social or policy mechanisms that are represented as the second indirect indicator level because the boundaries extend beyond the health determinants of newborn health. The second level indirect mechanisms are represented in the figure as twice removed from the IMR bulls-eye. These broader indirect mechanisms of IMR are the temporal last mile (i.e., the boundaries expand before conception and after birth). 


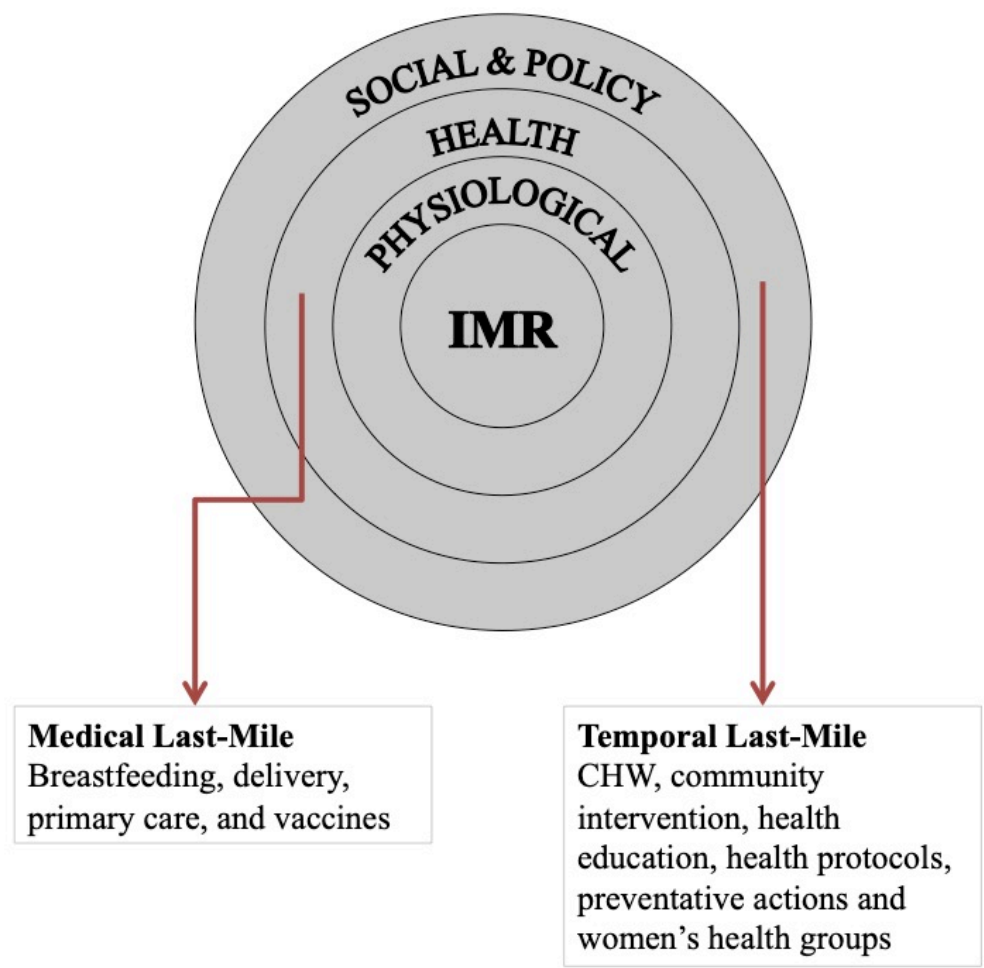

Figure 1: Three indicator levels of IMR- one direct, two indirect

The figure above shows the first direct indicator level of infant mortality along with the two indirect levels that alleviate the proximate causes of mortality. The above model is part of a broader model that will be discussed later in this chapter.

The direct or proximate physiological mechanisms of infant mortality The direct (or proximate) causes of infant mortality are caused by physiological (or medical) mechanisms ${ }^{45}$. These physiological mechanisms relate to the physical body, mind, and environment of mothers and their infants. The causes or factors of

\footnotetext{
45 James C. Cramer, "Social Factors and Infant Mortality: Identifying High-Risk Groups and Proximate Causes," Demography 24, no. 3 (August 1987): 299-322.
} 
infant mortality are complex; as such the physiological mechanisms can be roughly divided into both social and environmental factors, and biological factors ${ }^{46}$. Some examples of social and environmental factors that impact infant mortality include: education, ignorance, attitudes about medical care, availability of quality health care, legal barriers like discrimination and adolescent rights, access to welfare, marital status, race, family violence, and child care quality ${ }^{47}$. These social and environmental factors are less focused on the physical body as they are with where the physical body is and what it is doing. Some biological factors that impact infant mortality are maternal age, birth order, birth weight, prenatal care, maternal health, nutrition, maternal use of drugs, alcohol, or tobacco, and exposure to toxic substances $^{48}$. These biological factors shape the physical health of the body. All of the social and environmental, and biological factors by their absence, abundance, or misunderstanding of, are proximate causes of infant mortality, and they all ultimately relate to the physical health of mothers and their infants. Physiological mechanisms (or proximate causes) of infant mortality can be alleviated through indirect health, and social and policy mechanisms.

46 James C. Cramer, "Social Factors and Infant Mortality: Identifying High-Risk Groups and Proximate Causes," Demography 24, no. 3 (August 1987): 299-322.

${ }^{47}$ Cramer.

${ }^{48}$ Cramer. 
The indirect health mechanisms that alleviate the physiological mechanisms of infant mortality

Indirect health mechanisms can alleviate the proximate causes of infant mortality.

These indirect health mechanisms are actions and systems that impact the physiological mechanisms of infant mortality. The indirect health mechanisms identified for this paper are: breastfeeding, delivery, primary care, and vaccines. The following discussion from the literature highlights the importance of each in impacting the proximate mechanisms of IMR.

\section{Breastfeeding}

To reduce the infant mortality rate and ensure proper nutrition newborn babies should be exclusively breastfed ${ }^{49}$. From sub-Saharan Africa to Pakistan, exclusive breastfeeding has the potential to significantly improve the IMR ${ }^{505152}$. Exclusive breastfeeding means that "formula and/or solid, semi-solid or soft foods" are discouraged ${ }^{53}$. In preterm birth, particular attention to breastfeeding of the neonate can reduce infant mortality ${ }^{54}$ by preventing hypothermia and hypoglycaemia ${ }^{55}$.

\footnotetext{
49 Zulfa Abrahams, Zandile McHiza, and Nelia P Steyn, "Diet and Mortality Rates in Sub-Saharan Africa: Stages in the Nutrition Transition," BMC Public Health 11 (October 13, 2011): 4, https://doi.org/10.1186/1471-2458-11-801.

${ }^{50}$ Abrahams, McHiza, and Steyn, 4.

51 Saadia Riaz and Louise Condon, "The Experiences of Breastfeeding Mothers Returning to Work as Hospital Nurses in Pakistan: A Qualitative Study," Women and Birth, 2018, 2.

52 Abukari Issaka et al., "Factors Associated with Early Introduction of Formula and/or Solid, SemiSolid or Soft Foods in Seven Francophone West African Countries," Nutrients 7, no. 2 (2015): 949.

53 Issaka et al., 949.

54 JE Lawn et al., "Newborn Survival in Low Resource Settings-Are We Delivering?," BJOG An International Journal of Obstetrics and Gynaecology 116 (2009): 54.
} 
Babies in the later neonatal phase can also benefit enormously from exclusive breastfeeding because it helps protect against common infections that are the leading cause of infant deaths in developing countries ${ }^{56}$. Promoting exclusive breastfeeding is a low technology solution to a pressing issue. Breastfeeding can be promoted in a community setting ${ }^{57}$ as part of prenatal or postnatal care and exclusive breastfeeding can be promoted at facility births. Regardless of where the message is shared, the important aspect to communicate to families is that breastfeeding is necessary and educating women about the benefits of breastfeeding and then supporting women in the endeavor is key to lowering the IMR in developing countries. Educating men about the benefits of exclusive breastfeeding is important too because they can support and encourage women to breastfeed rather than other practices ${ }^{58}$. In order for children to have the "best start in life" health practitioners must be made aware how vitality important it is to "protect, promote, and support breastfeeding 5960 . Exclusive breastfeeding, along with other "evidence-

\footnotetext{
55 Sandra L. Huffman, Elizabeth R. Zehner, and Cesar Victora, "Can Improvements in Breast-Feeding Practices Reduce Neonatal Mortality in Developing Countries?," Midwifery 17, no. 2 (June 1, 2001): 80, https://doi.org/10.1054/midw.2001.0253.

56 Huffman, Zehner, and Victora, 80.

57 Karen M. Edmond et al., "Aetiology of Stillbirths and Neonatal Deaths in Rural Ghana: Implications for Health Programming in Developing Countries," Paediatric \& Perinatal Epidemiology 22, no. 5 (September 2008): 436, https://doi.org/10.1111/j.1365-3016.2008.00961.x.

58 Marie Guadagno, Michael Mackert, and Aaron Rochlen, "Improving Prenatal Health: Setting the Agenda for Increased Male Involvement," American Journal of Men's Health 7, no. 6 (2013): 524, https://doi.org/10.1177/1557988313490785.

${ }^{59}$ Randa Jarudi Saadeh, "The Baby-Friendly Hospital Initiative 20 Years On: Facts, Progress, and the Way Forward," Journal of Human Lactation 28, no. 3 (August 1, 2012): 272, https://doi.org/10.1177/0890334412446690.
} 
based available interventions" like tetanus toxoid immunization for mothers, quality care at delivery; newborn resuscitation, proper cord care, and neonatal infection management could prevent nearly $50 \%$ of newborn deaths ${ }^{61}$. Another study reveals that infants that are not breastfed have "seven-fold and five-fold increased risks of death from diarrhea and pneumonia, respectively, compared with infants who are exclusively breastfed"62.

\section{Delivery}

The birth of an infant occupies a short time in the continuum of care of mothers, and babies. Even so, this time frame accounts for $40 \%$ of maternal deaths and approximately 5.5 million stillbirths and neonatal deaths ${ }^{63}$. The necessity to get delivery right (i.e., quality health protocols, skilled attendance, hygienic practices, and access to emergency obstetric care ${ }^{64}$ ) is of vital importance if the infant mortality rate is to improve. Studies show that countries with the highest neonatal mortality rates are those where "skilled attendance and institutional delivery rates are lowest". ${ }^{65}$ Improved delivery reduces neonatal mortality, intrapartum stillbirths,

60 "Global Strategy: Breastfeeding Critical for Child Survival--UNICEF and WHO Call for Increased Commitment to Appropriate Feeding Practices for All Infants and Young Children," Indian Journal Of Medical Sciences 58, no. 3 (March 2004): 138.

61 Zohra S. Lassi and Zulfiqar A. Bhutta, “Community-Based Intervention Packages for Reducing Maternal and Neonatal Morbidity and Mortality and Improving Neonatal Outcomes," Cochrane Database of Systematic Reviews, no. 3 (2015): 1.

62 Black, Morris, and Bryce, “Where and Why Are 10 Million Children Dying Every Year?," 2227.

63 Lawn et al., "Every Newborn,” 189.

${ }^{64}$ Lawn et al., "Newborn Survival in Low Resource Settings-Are We Delivering?," 55.

65 Joy E Lawn, Simon Cousens, and Jelka Zupan, “4 Million Neonatal Deaths: When? Where? Why?, Lancet (London, England) 365, no. 9462 (March 5, 2005): 896. 
and half a million maternal deaths every year ${ }^{66}$. At delivery, three causes

(infections- sepsis, pneumonia, meningitis; "intrapartum related deaths or 'birth asphyxia'; and preterm birth complications) constitute $86 \%$ of worldwide newborn deaths ${ }^{67}$. Significant improvements in the IMR can be made through "existing programmes" through appropriate prenatal care, delivery and antenatal care ${ }^{68}$. Reducing the number of home births by providing access and transportation to an appropriate birth facility improves outcomes for newborns ${ }^{69}$. Additionally, facility birth doubles the chances of an infant being immunized, the outcome of which transcends beyond surviving delivery but provides lifelong health outcomes ${ }^{70}$. Evidence based interventions like maternal tetanus toxoid immunization, hygienic care, newborn resuscitation, exclusive breastfeeding, clean cord care, infection management in newborns in facilities and communities at time of delivery can prevent nearly half of neonatal deaths ${ }^{71}$.

\footnotetext{
${ }^{66}$ Joy Lawn, Kenji Shibuya, and Claudia Stein, "No Cry at Birth: Global Estimates of Intrapartum Stillbirths and Intrapartum-Related Neonatal Deaths," Bulletin of the World Health Organization 83 (June 2005): 413, https://doi.org/10.1590/S0042-96862005000600008.

${ }^{67}$ Lawn et al., "Newborn Survival in Low Resource Settings-Are We Delivering?," 52.

${ }^{68}$ Lawn et al., 52.

${ }^{69}$ Seni Kouanda et al., "Home Births in the Context of Free Health Care: The Case of Kaya Health District in Burkina Faso," International Journal of Gynecology \& Obstetrics, Maternal and Neonatal Health in Africa at MDG End: Availability of and Access to Maternal Health Services, and Outcomes of Intervention Strategies, 135 (November 1, 2016): S39, https://doi.org/10.1016/j.ijgo.2016.08.009. ${ }^{70}$ Cheryl A. Moyer, Lia Tadesse, and Senait Fisseha, "The Relationship between Facility Delivery and Infant Immunization in Ethiopia," International Journal of Gynecology \& Obstetrics 123, no. 3 (December 1, 2013): 217-20, https://doi.org/10.1016/j.ijgo.2013.06.030.

${ }^{71}$ Lassi and Bhutta, "Community-Based Intervention Packages for Reducing Maternal and Neonatal Morbidity and Mortality and Improving Neonatal Outcomes," 1.
} 


\section{Primary Care}

Primary care is a "health service delivery approach characterized by first-contact care, ease of access, care for a broad range of health needs, continuity, and the involvement of family and community"72. Advocated for since the Declaration of Alma Ata in 1978 to expand health services in developing countries ${ }^{73}$, primary care has had success in improving the IMR in Brazil74, is instrumental for the same in Bangladesh ${ }^{75}$, and has demonstrated a $41 \%$ IMR decline in a primary care intervention area in Bolivia in the $1990 \mathrm{~s}^{76}$. The primary care model often seeks to expand into previously unreached rural populations to deliver essential services with targeted health interventions ${ }^{77}$. Primary care expansion provides greater access for pregnant women before, during, and after delivery, as well as provides education for new mothers in developing countries. Community-based primary care

\footnotetext{
72 Margaret Elizabeth Kruk et al., "The Contribution of Primary Care to Health and Health Systems in Low- and Middle-Income Countries: A Critical Review of Major Primary Care Initiatives," Social Science \& Medicine 70, no. 6 (March 1, 2010): 904, https://doi.org/10.1016/j.socscimed.2009.11.025. 73 Kruk et al., 904.

74 James Macinko et al., "Going to Scale with Community-Based Primary Care: An Analysis of the Family Health Program and Infant Mortality in Brazil, 1999-2004," Social Science \& Medicine 65, no. 10 (November 2007): 17, https://doi.org/10.1016/j.socscimed.2007.06.028.

75 Subir Saha and M. Kabir, "Survival of Newborns: Implications for Achieving the Millennium Development Goal in Bangladesh," World Health \& Population 11, no. 1 (2009): 5-13, https://doi.org/10.12927/whp.2009.20990.

${ }^{76}$ Henry B. Perry, David S. Shanklin, and Dirk G. Schroeder, "Impact of a Community-Based Comprehensive Primary Healthcare Programme on Infant and Child Mortality in Bolivia," Journal of Health, Population and Nutrition 21, no. 4 (December 2003): 389.

77 Lars Åke Persson et al., “Child Survival Revolutions Revisited - Lessons Learned from Bangladesh, Nicaragua, Rwanda and Vietnam," Acta Paediatrica 106, no. 6 (June 2017): 873, https://doi.org/10.1111/apa.13830.
} 
service can improve health outcomes for children in poor countries by expanding or using the existing health venues and strengthening that quality of care ${ }^{78}$.

\section{Vaccines}

Maternal vaccination programs, coupled with other interventions have led to declines in under-5 child mortality ${ }^{79}$ as well as neonates ${ }^{80}$. Tetanus toxoid vaccination as part of antenatal interventions in mothers can improve infant survival81. Babies should also receive essential preventative vaccination like BCG and Hepatitis B, and vitamin $\mathrm{K}^{8283}$. Vaccination is a low technology solution that can be implemented in both community and facility settings to improve health outcomes for babies ${ }^{84}$. Tetanus in neonates can occur within the first 28 days and fatality rates of cases are nearly $100 \%$ in developing countries ${ }^{85}$ for the unvaccinated but two or

\footnotetext{
${ }^{78}$ Kruk et al., "The Contribution of Primary Care to Health and Health Systems in Low- and MiddleIncome Countries."

${ }^{79}$ Haidong Wang et al., "Global, Regional, National, and Selected Subnational Levels of Stillbirths, Neonatal, Infant, and under-5 Mortality, 1980-2015: A Systematic Analysis for the Global Burden of Disease Study 2015," The Lancet 388, no. 10053 (October 2016): 1760, https://doi.org/10.1016/S0140-6736(16)31575-6.

${ }^{80}$ Edmond et al., "Aetiology of Stillbirths and Neonatal Deaths in Rural Ghana," 436.

${ }^{81} \mathrm{~L}$ McKenzie and M Ellis, "Community-Based Interventions to Improve Neonatal Survival in LowResource Settings," Annals Of Tropical Paediatrics 31, no. 3 (2011): 192, https://doi.org/10.1179/1465328111Y.0000000002.

82 "Newborns: Reducing Mortality," accessed March 25, 2019, https://www.who.int/newsroom/fact-sheets/detail/newborns-reducing-mortality.

83 Gary L Darmstadt et al., "A Strategy for Reducing Maternal and Newborn Deaths by 2015 and Beyond," BMC Pregnancy and Childbirth 13, no. 1 (December 2013): 3, https://doi.org/10.1186/1471-2393-13-216.

84 Edmond et al., "Aetiology of Stillbirths and Neonatal Deaths in Rural Ghana," 436.

${ }^{85}$ Alison Delano Ridpath et al., "Progress towards Achieving and Maintaining Maternal and Neonatal Tetanus Elimination in the African Region," The Pan African Medical Journal 27, no. Suppl 3 (2017): 2, https://doi.org/10.11604/pamj.supp.2017.27.3.11783.
} 
more doses of the vaccination can prevent neonatal death ${ }^{86}$. Immunization in childhood of three doses of DTP (diphtheria, tetanus toxoid, and pertussis) is not considered enough to prevent maternal and neonatal tetanus during a woman's childbearing years; additional tetanus toxoid vaccination is required ${ }^{87}$.

The indirect social and policy mechanisms that alleviate the physiological mechanisms of infant mortality While the four indirect health mechanisms can alleviate the proximate causes of the infant mortality rate, the literature is also fairly insistent that it requires more than just health mechanisms, or having a doctor in attendance at delivery. The indirect social and policy mechanisms, although slightly removed from the delivery room, also impact the proximate physiological causes of infant mortality. From the literature, this paper has identified the following indirect social and policy mechanisms that alleviate the proximate causes of infant mortality, they are: community health workers, health education, community interventions, women's health groups, preventative actions against risk factors and health protocols. The following discussion highlights the importance of each in alleviating the proximate causes of infant mortality.

\footnotetext{
${ }^{86}$ Vittorio Demicheli, Antonella Barale, and Alessandro Rivetti, "Vaccines for Women for Preventing Neonatal Tetanus," Cochrane Database of Systematic Reviews, no. 7 (2015): 972, https://doi.org/10.1002/14651858.CD002959.pub4.

87 J Vandelaer, "Tetanus in Developing Countries: An Update on the Maternal and Neonatal Tetanus Elimination Initiative," Vaccine 21, no. 24 (July 28, 2003): 3443, https://doi.org/10.1016/S0264410X(03)00347-5.
} 


\section{Community Health Workers}

The World Health Organization recommends ${ }^{88}$ that all pregnant women have a skilled health worker in attendance during delivery ${ }^{89}$. In many instances in the developing world women do not have an attended birth, or they will have a female family member attend the birth in the home, or a traditional birth attendant (TBA) may be present to assist. The TBA is "controversial" but should not be ruled out if "partnerships with the health system" can be bolstered ${ }^{90}$. The community health worker (CHW), on the other hand, is an accessible and positive step towards quality, informed care that can treat women and intervene with immunizations and helpful information surrounding neonatal problems. Family planning ${ }^{91}$, clean delivery, cord care, thermal care, counseling, breastfeeding, and facility birth promotion are typically addressed by $\mathrm{CHWs}^{92}$. Incorporating a community health worker (CHW) into the childbirth dynamic is a crucial step in providing quality, skilled care to mother and baby. In low resource countries, community health workers can improve service delivery during pregnancy and delivery, and improve neonatal

\footnotetext{
88 Jody R. Lori and Joyceen S. Boyle, "Cultural Childbirth Practices, Beliefs, and Traditions in Postconflict Liberia," Health Care for Women International 32, no. 6 (2011): 456, https://doi.org/10.1080/07399332.2011.555831.

89 Gary L. Darmstadt et al., "60million Non-Facility Births: Who Can Deliver in Community Settings to Reduce Intrapartum-Related Deaths?," International Journal of Gynecology \& Obstetrics, IntrapartumRelated Deaths: Evidence for Action, 107 (October 1, 2009): S89, https://doi.org/10.1016/j.ijgo.2009.07.010.

90 Darmstadt et al., S89.

91 Jaime Haver et al., "Experiences Engaging Community Health Workers to Provide Maternal and Newborn Health Services: Implementation of Four Programs," International Journal of Gynecology \& Obstetrics, Advancing Implementation in Maternal and Newborn Health, 130 (June 1, 2015): S37-38, https://doi.org/10.1016/j.ijgo.2015.03.006.

92 McKenzie and Ellis, "Community-Based Interventions to Improve Neonatal Survival in LowResource Settings," 193.
} 
outcomes ${ }^{93}$, especially at the "household level"94. Community level participation in women's groups is very important because it can further integrate the CHW into accepted practice in the lives and homes of women individually. Together, community action and CHWs have a reinforcing impact that lowers the IMR, particularly for neonates ${ }^{9596}$. Women that have access to a CHW are encouraged to seek additional care for their newborns when necessary and health outcomes and IMR is improved ${ }^{97}$. The community health worker is rather different from the rest of the indirect social and policy mechanisms (outlined below) because the CHW conveys action and support of the other vital indirect social and policy mechanisms while also being an essential component of improving IMR decline.

\section{Community Interventions}

Community interventions, much like preventative actions, can occur throughout the care cycle of women in their child bearing years and before. Community interventions that occur earlier in the care cycle of pregnant women require

\footnotetext{
${ }^{93}$ Gary L. Darmstadt et al., "60 Million Non-Facility Births: Who Can Deliver in Community Settings to Reduce Intrapartum-Related Deaths?," International Journal of Gynecology \& Obstetrics 107, no. Supplement (October 2009): S89, https://doi.org/10.1016/j.ijgo.2009.07.010.

${ }_{94}$ Zulfiqar A. Bhutta et al., "Reducing Maternal, Newborn, and Infant Mortality Globally: An Integrated Action Agenda," International Journal of Gynecology \& Obstetrics 119, no. 1 (2012): S16, https://doi.org/10.1016/j.ijgo.2012.04.001.

${ }^{95}$ Rajiv Bahl et al., "Why Is Continuum of Care from Home to Health Facilities Essential to Improve Perinatal Survival?," Seminars in Perinatology 34, no. 6 (December 2010): 477, https://doi.org/10.1053/j.semperi.2010.09.001.

${ }^{96}$ McKenzie and Ellis, "Community-Based Interventions to Improve Neonatal Survival in LowResource Settings," 191.

${ }^{97}$ Christine Kayemba Nalwadda et al., "High Compliance with Newborn Community-to-Facility Referral in Eastern Uganda: An Opportunity to Improve Newborn Survival," PLOS ONE 8, no. 11 (November 29, 2013): e81610, https://doi.org/10.1371/journal.pone.0081610.
} 
different levels of health knowledge, and different levels of health response than interventions later in in the care cycle of pregnant women. Addressing potential challenges earlier, rather than later, has its advantages in that it is often less dramatic a response. Highly technical interventions were once considered the primary way to save infants; however, new evidence suggests that interventions at the community level at home are low cost and effective ${ }^{98}$. Community-based interventions can improve infant survival in low-resource countries by addressing common health concerns through culturally and logistically appropriate means ${ }^{99}$. Specific health low-technology community interventions can include: improving hygiene, vaccination, improving nutrition through appropriate supplementation like folic acid or vitamin K, promoting exclusive breastfeeding for infants ${ }^{100}$, family planning, and improved access to primary care ${ }^{101}$. Neonatal mortality could be almost halved based on proven community interventions as discussed. ${ }^{102}$

\footnotetext{
${ }^{98}$ McKenzie and Ellis, "Community-Based Interventions to Improve Neonatal Survival in LowResource Settings," 192.

${ }^{99}$ Nirmala Nair et al., "Mobilizing Women's Groups for Improved Maternal and Newborn Health: Evidence for Impact, and Challenges for Sustainability and Scale Up," International Journal of Gynecology \& Obstetrics, World Report on Women's Health 2012:, 119 (October 1, 2012): S22, https://doi.org/10.1016/j.ijgo.2012.03.014.

${ }^{100}$ Edmond et al., "Aetiology of Stillbirths and Neonatal Deaths in Rural Ghana," 436.

${ }^{101}$ Haver et al., "Experiences Engaging Community Health Workers to Provide Maternal and Newborn Health Services," S37-38.

102 Lassi and Bhutta, "Community-Based Intervention Packages for Reducing Maternal and Neonatal Morbidity and Mortality and Improving Neonatal Outcomes," 1.
} 
Community interventions are not always health related but often focus on

alleviating poverty and improving access to education ${ }^{103}$.

New technology such as mobile health services can facilitate life saving community intervention for infants by sharing medical information with caregivers at remote sites, or mitigating risks during travel to a health facility ${ }^{104}$, recognizing that the type of community intervention available is dependent on the "proximity to a woman's home" versus a quality health facility ${ }^{105}$.

\section{Health Education}

Health education is an essential component of improving the IMR in developing countries. Without health education, human capital is wasted, self-determination is curtailed and large percentages of populations are excluded from the labor market as well as advances in the well being of society. Research continually links maternal education with child mortality levels ${ }^{106107}$. Without education, men and women may rely solely on traditional or cultural norms regarding what constitutes a healthy

\footnotetext{
103 Zulfiqar A. Bhutta et al., "Community-Based Interventions for Improving Perinatal and Neonatal Health Outcomes in Developing Countries: A Review of the Evidence," Pediatrics 115, no. 2 Suppl (2005): 522, https://doi.org/10.1542/peds.2004-1441.

104 Peter von Dadelszen et al., "Moving beyond Silos: How Do We Provide Distributed Personalized Medicine to Pregnant Women Everywhere at Scale? Insights from PRE-EMPT," International Journal of Gynecology \& Obstetrics, World Report on Women's Health 2015: The unfinished agenda of women's reproductive health, 131 (October 1, 2015): S10, https://doi.org/10.1016/j.ijgo.2015.02.008.

105 Nirmala Nair et al., "Improving Newborn Survival in Low-Income Countries: Community-Based Approaches and Lessons from South Asia," PLoS Medicine 7, no. 4 (2010): 2.

${ }^{106}$ Emmanuela Gakidou et al., "Increased Educational Attainment and Its Effect on Child Mortality in 175 Countries between 1970 and 2009: A Systematic Analysis," The Lancet 376, no. 9745 (2010): 959-974.

107 David M. Cutler and Adriana Lleras-Muney, "Education and Health: Evaluating Theories and Evidence" (National bureau of economic research, 2006), 9.
} 
lifestyle and/or pregnancy, and care for infants. From preconception to care of the newborn infant, health education allows men and women to participate in life saving practices and curb harmful practices. Health education is not an exclusive prescription for pregnant women, but also for men, children, communities, and medical professionals ${ }^{108}$.

Maternal illiteracy increases the chances of infant mortality in both developing and developed countries. Maternal illiteracy is often coupled with low socioeconomic status, which works against improving the IMR in countries. While both are problematic, illiteracy is more closely linked to infant death than socioeconomic status $^{109}$. A study from India also notes a similar pattern of decreased IMR with additional education: every year of school a girl receives, a corresponding ten percent drop in infant mortality risk can be expected ${ }^{110}$. Beyond improving literacy and general education levels, specific health education that relates to a healthy pregnancy and newborn care is essential. Skin-to-skin-contact (STSC), or kangaroo care as it is also called, is an important practice of holding newborns (in particular low-birth weight babies) against the skin of the mother or father to keep the baby warm and thriving. In many low-resource settings this very simple, low technology practice supports new born life and is an easy way to improve health outcomes,

\footnotetext{
108 Guadagno, Mackert, and Rochlen, "Improving Prenatal Health," 525.

${ }^{109}$ S. Jahan and S. Jahan, "Evidence-Based Public Health Policy and Practice: Poverty and Infant Mortality in the Eastern Mediterranean Region: A Meta-Analysis," Journal of Epidemiology and Community Health (1979-) 62, no. 8 (2008): 749.

110 R. Amonoo-Lartson, District Health Care: Challenges for Planning, Organisation, and Evaluation in Developing Countries (London: Macmillan Press, 1984).
} 
especially if care takers are educated to understand the benefits associated with it ${ }^{111}$. Pre- and post-natal health education of a patient helps "influence" maternal postpartum behavior and encourages life saving behaviors for infants such as breastfeeding and infant care $\mathrm{c}^{112113114}$. Increasingly, men are seen as part of the solution to improve IMR. When men are receive health education and are involved they help their partners achieve better health outcomes by facilitating transportation, identifying health abnormalities, and encouraging positive behavior like reminding their partner to take antiretroviral medications if indicated to reduce HIV transmission ${ }^{115}$. If men receive health education about the advantages of care seeking for pregnant women then they encourage rather than discourage the practice, and the IMR improves ${ }^{116117}$. Communities are also foundational for learning, and health education often occurs in participatory settings within villages, not necessarily within schools. The health education venue might be a make shift literacy hut, chairs huddled in a circle in a

\footnotetext{
111 Alessandra Bazzano et al., "Introducing Home Based Skin-to-Skin Care for Low Birth Weight Newborns: A Pilot Approach to Education and Counseling in Ghana," Global Health Promotion 19, no. 3 (2012): 42.

112 Julie Maslowsky et al., "Effects of Postpartum Mobile Phone-Based Education on Maternal and Infant Health in Ecuador," International Journal of Gynecology \& Obstetrics 134, no. 1 (July 1, 2016): 93, https://doi.org/10.1016/j.ijgo.2015.12.008.

113 Shin Chao et al., "Integrated Approaches to Improve Birth Outcomes: Perinatal Periods of Risk, Infant Mortality Review, and the Los Angeles Mommy and Baby Project," Maternal and Child Health Journal 14, no. 6 (2010): 836, https://doi.org/10.1007/s10995-010-0627-2.

114 McKenzie and Ellis, "Community-Based Interventions to Improve Neonatal Survival in LowResource Settings," 192.

115 Guadagno, Mackert, and Rochlen, "Improving Prenatal Health," 524.

116 Guadagno et al.

117 Anthony K. Mbonye, "Prevalence of Childhood Illnesses and Care-Seeking Practices in Rural Uganda," Research article, The Scientific World Journal, 2003, 729, https://doi.org/10.1100/tsw.2003.52.
} 
shared space or at a woman's home. The community setting allows women, who might not have attended school, and others to learn essential health skills necessary for pregnancy that improve the mortality rate of infants. A mother can learn that having a series of tetanus toxoid vaccinations before birth can save her baby's life in the event of sub-optimal cord care or lack of clean delivery practices at delivery that can lead to death ${ }^{118119}$. The community environment can lend authority to the educational message, or the acceptance of the new information, and discourage potentially harmful traditional practices ${ }^{120121}$. Communities, instead of health clinics, tend to be the places where "antenatal, delivery, and postnatal experiences for women usually take place" in developing countries ${ }^{122}$. But, traditional educational settings are useful. For instance, the Government of Eritrea has used schools to reach remote areas and "implement health interventions by training teachers to be allies for health workers"123. Health education is also necessary to build a larger cadre of medical professionals. From community health workers to obstetric surgeons, more health workers are

\footnotetext{
118 McKenzie and Ellis, "Community-Based Interventions to Improve Neonatal Survival in LowResource Settings," 192.

${ }^{119}$ Demicheli, Barale, and Rivetti, "Vaccines for Women for Preventing Neonatal Tetanus."

120 Elizabeth A. Goodburn, Rukhsana Gazi, and Mushtaque Chowdhury, "Beliefs and Practices Regarding Delivery and Postpartum Maternal Morbidity in Rural Bangladesh," Studies in Family Planning 26, no. 1 (1995): 22-32, https://doi.org/10.2307/2138048.

121 Jasper Chiwuzie and Chike Okolocha, "Traditional Belief Systems and Maternal Mortality in a Semi-Urban Community in Southern Nigeria," African Journal of Reproductive Health / La Revue Africaine de La Santé Reproductive 5, no. 1 (2001): 75-82, https://doi.org/10.2307/3583200. 122 Mikey Rosato et al., "Community Participation: Lessons for Maternal, Newborn, and Child Health," The Lancet 372, no. 9642 (September 2008): 962, https://doi.org/10.1016/S0140-6736(08)614063.

123 United Nations, "Innovations Driving Health MDGS in Eritrea," Health Millennium Development Goals Report Abridged Version (United Nations, September 2014), 16.
} 
required to meet international guidelines of patient/doctor ratios for healthy infants $^{124125}$. Many developing countries have a dearth of medical professionals so substantially scaling up the medical community through health education is essential to meet the needs of communities ${ }^{126127}$. The increased number of doctors does not always translate to immediate improvements in the IMR because frequently the doctors are far removed from primary care where most women interact with health services but it does work towards the goal of improving some levels of health protocols.

\section{Health Protocols}

Improving health protocols of existing neonatal health care is necessary to save infant lives ${ }^{128}$. Following recommended health protocols, from health care worker knowledge to the hygienic conditions of health facilities, is poised to make a significant change in the IMR of countries because health infrastructure is already in place. Rather than creating new health systems, the existing ones that currently

\footnotetext{
124 Wim Van Lerberghe et al., "Country Experience with Strengthening of Health Systems and Deployment of Midwives in Countries with High Maternal Mortality," The Lancet 384, no. 9949 (2014): 1217.

125 Petra ten Hoope-Bender et al., "Improvement of Maternal and Newborn Health through Midwifery," The Lancet 384, no. 9949 (2014): 1229.

126 Van Lerberghe et al., "Country Experience with Strengthening of Health Systems and Deployment of Midwives in Countries with High Maternal Mortality."

127 Barbara Deller et al., "Task Shifting in Maternal and Newborn Health Care: Key Components from Policy to Implementation," International Journal of Gynecology \& Obstetrics, Advancing Implementation in Maternal and Newborn Health, 130 (June 1, 2015): S30, https://doi.org/10.1016/j.ijgo.2015.03.005.

128 Cesar Gomes Victora and Fernando Celso Barros, "Infant Mortality Due to Perinatal Causes in Brazil: Trends, Regional Patterns and Possible Interventions," São Paulo Medical Journal 119, no. 1 (2001): 33-42.
} 
interact with pregnant women and their infants can level up through globally recognized health protocols via training, technology, and improved hygiene standards. Facility births have almost reached $80 \%$ worldwide; there is an opportunity to provide better newborn care at this intervention point ${ }^{129}$. If certain health protocols are upgraded, more women could be inclined to utilize existing health facilities. ${ }^{130}$ Improvement can be achieved through "drills and audits"131. Or, for births already occurring in facilities, "36\% of intrapartum-deaths" could be avoided if extra attention was placed on following appropriate health protocols ${ }^{132}$. If health protocols are recognized and acknowledged by pregnant women perhaps they will be more willing to seek care at health facilities at the beginning of labor rather than holding off in hopes of avoiding delivery at a facility all together ${ }^{133}$. Arriving sooner, rather than later, and having access to "high-quality emergency obstetric care"134135 can save newborn lives. In 2016, approximately 1 million newborns died within the first 24 hours of life, with $75 \%$ of all newborn deaths

\footnotetext{
129 "Newborns."

130 Günther Fink, Rebecca Ross, and Kenneth Hill, "Institutional Deliveries Weakly Associated with Improved Neonatal Survival in Developing Countries: Evidence from 192 Demographic and Health Surveys," International Journal of Epidemiology 44, no. 6 (December 1, 2015): 1887, https://doi.org/10.1093/ije/dyv115.

131 Joy E. Lawn et al., "Reducing Intrapartum-Related Deaths and Disability: Can the Health System Deliver?," International Journal of Gynecology \& Obstetrics 107, no. Supplement (2009): S123.

132 Lawn et al., S123.

133 Karen Ballard et al., "The Effect of Prenatal and Intrapartum Care on the Stillbirth Rate among Women in Rural Ethiopia," International Journal of Gynecology \& Obstetrics 133, no. 2 (2016): 167. 134 Darmstadt et al., "60million Non-Facility Births," S89.

135 Saha and Kabir, "Survival of Newborns," 13.
} 
occurring within the first week ${ }^{136}$. Ensuring that appropriate health protocols are known and available can improve the IMR. About half of maternal deaths in a region of Ghana happen within 24 hours of being admitted at a hospital ${ }^{137}$. If the mother dies, the chances of survival of the newborn are diminished, particularly in developing countries. In order to realize improvements in the IMR, following appropriate health protocols throughout the continuum of care are required ${ }^{138}$. Changing health protocols necessitates facility level commitment to implementing known quality strategies. Saving the lives of babies is not "new science" rather it is the implementation of health protocol science that is already known ${ }^{139}$.

\section{Preventative Actions Against Risk Factors}

A focus on preventative actions against risk factors throughout a woman's childbearing years can significantly reduce infant mortality ${ }^{140}$. Preventative actions ideally begin preconception with appropriate family planning but frequently preventative action begins much later in the care cycle of a pregnant woman.

\footnotetext{
136 "Newborns."

137 Kofi Issah, Alexis Nang-Beifubah, and Chris F. Opoku, "Maternal and Neonatal Survival and Mortality in the Upper West Region of Ghana," International Journal of Gynecology \& Obstetrics 113, no. 3 (June 1, 2011): 208, https://doi.org/10.1016/j.ijgo.2011.01.007.

138 ten Hoope-Bender et al., "Improvement of Maternal and Newborn Health through Midwifery," 1266.

${ }^{139}$ Lawn et al., "Newborn Survival in Low Resource Settings-Are We Delivering?," 57.

140 Adewale Troutman, "Addressing U.S. Infant Mortality through Healthy Start Programs," Nation's Health 43, no. 2 (March 2013): 3-3.
} 
Preventing an "insult"141, or compromise of health, in the first place (primary prevention) reduces life-threatening complications for mother and child. "Primary prevention" can be achieved before, during, and after pregnancy by attending to maternal health and nutritional status, monitoring for pregnancies that are at risk (i.e., potentially underweight babies ${ }^{142}$ ) attendance at birth by skilled health workers, and early management of obstetric emergencies ${ }^{143}$. "Secondary prevention" occurs after an event, i.e. the delivery of the infant, with "resuscitation of the 'nonbreathing' neonate"144. "Tertiary prevention" includes managing significant complications that are a result, often, from the secondary insult. Tertiary prevention requires complex levels of care that even "well-resourced health systems" find challenging ${ }^{145}$. Primary prevention of challenges is likely to impact the IMR the most as second and tertiary prevention is harder to implement in low resource settings ${ }^{146}$. There is much to gain from preventing dire circumstances. Prepregnancy risk factors to manage are the age of first birth for a mother, the birth spacing between pregnancies, birth order, and nutritional status ${ }^{147}$. Access to clean water, maternal health, antenatal care, vaccination and education are other forms of

\footnotetext{
141 Joy E. Lawn et al., "Two Million Intrapartum-Related Stillbirths and Neonatal Deaths: Where, Why, and What Can Be Done?," International Journal of Gynecology \& Obstetrics, Intrapartum-Related Deaths: Evidence for Action, 107 (October 1, 2009): S13, https://doi.org/10.1016/j.ijgo.2009.07.016. 142 Joan Maxwell, "The Prevention of Prematurity: A Strategy to Reduce Infant Mortality in the District of Columbia.," 1982.

143 Lawn et al., "Two Million Intrapartum-Related Stillbirths and Neonatal Deaths."

144 Lawn et $\underline{\text { al. }} . \underline{\underline{S}} \underline{\mathrm{S}} \underline{\underline{3}}$.

145 Lawn et al., S13.

146 Lawn et al., S13.

147 Lawn et al., S13.
} 
primary prevention ${ }^{148}$. Prevention can reduce premature birth, vertical HIV transmission ${ }^{149150}$, birth asphyxia and early newborn death from infections (sepsis, pneumonia, meningitis) ${ }^{151}$. A study in India indicates that the "prevention and/or management of neonatal infections will reduce neonatal mortality by 40 to $50 \%$ "152. Preventative actions against risk factors get ahead of infant mortality by minimizing the opportunity for compromised circumstances that threaten mother and child in the first place.

\section{Women's Health Groups}

While community interventions are useful and necessary, participation by women in women's health groups is extremely important. The community intervention is something that is done for and/or to a woman, whereas women's health groups are something women can do for themselves to improve health outcomes for their infants. The act of participating in a group with other women that is led by a local, or culturally appropriate individual has a significant impact because it demonstrates a level of autonomy and interest in changing the status quo, i.e., poor health outcomes.

\footnotetext{
148 Lawn et al., "Newborn Survival in Low Resource Settings-Are We Delivering?"

149 Tanya Doherty et al., "Implications of the New WHO Guidelines on HIV and Infant Feeding for Child Survival in South Africa," Bulletin Of The World Health Organization 89, no. 1 (January 1, 2011): 62-67, https://doi.org/10.2471/BLT.10.079798.

150 Anisa Ghadrshenas et al., "Improved Access to Early Infant Diagnosis Is a Critical Part of a ChildCentric Prevention of Mother-to-Child Transmission Agenda," Aids 27 (2013): S197-S205.

151 Lawn et al., "Newborn Survival in Low Resource Settings-Are We Delivering?"

152 Abhay T Bang et al., "Why Do Neonates Die in Rural Gadchiroli, India? (Part II): Estimating Population Attributable Risks and Contribution of Multiple Morbidities for Identifying a Strategy to Prevent Deaths," Journal of Perinatology 25, no. S1 (March 2005): S35, https://doi.org/10.1038/sj.jp.7211270.
} 
One study in rural Nepal shows that women's groups “convened by a local woman facilitator, reduced neonatal mortality by $30 \%{ }^{\prime \prime 153}$. Through local female leaders, women's health groups address concerns about infant mortality and strategize how to improve outcomes within the community ${ }^{154}$. Participating in women's health groups prior to delivery has positive impacts on IMR 155 , not only after the fact when women learn about healthy nutrition and vaccinations for their infants. Analysis also demonstrates that energized participatory community groups can increase the number of facility births and prevent " 1 out of 3 early neonatal deaths" 156 . So while communities are typically the place where the entire cycle of maternal care and delivery occurs ${ }^{157}$ in rural and low resource countries, participation in women's health groups can improve the IMR by encouraging facility births if possible when indicated. A key to the improved IMR might be the combined impact of a community health worker and the acceptance of this worker due to participation in women's

\footnotetext{
153 Dharma S. Manandhar et al., "Effect of a Participatory Intervention with Women's Groups on Birth Outcomes in Nepal: Cluster-Randomised Controlled Trial," The Lancet 364, no. 9438 (2004): 976. 154 Prasanta Tripathy et al., "Effect of a Participatory Intervention with Women's Groups on Birth Outcomes and Maternal Depression in Jharkhand and Orissa, India: A Cluster-Randomised Controlled Trial," The Lancet 375, no. 9721 (2010): 1182.

155 Intisar Khanani et al., "The Impact of Prenatal WIC Participation on Infant Mortality and Racial Disparities," American Journal of Public Health 100, no. S1 (April 2, 2010): 204, https://doi.org/10.2105/AJPH.2009.168922.

${ }^{156}$ Anne CC Lee et al., "Linking Families and Facilities for Care at Birth: What Works to Avert Intrapartum-Related Deaths?," International Journal of Gynecology \& Obstetrics, Intrapartum-Related Deaths: Evidence for Action, 107 (October 1, 2009): S82, https://doi.org/10.1016/j.ijgo.2009.07.012. 157 Rosato et al., “Community Participation," 962.
} 
groups ${ }^{158}$ as well as following established health protocols. Participation in a woman's health group demonstrates a level of agency.

A review of literature also reveals IMR improvement in low resource settings is not necessarily the result of a single medical intervention ${ }^{159}$, but instead, appropriate care is delivered over time. The concepts, techniques and strategies known to improve the IMR are essentially low technology activities, and if implemented correctly over time, can improve maternal and infant health outcomes. However, just because something is known does not make it easy. Multiple, cross-cutting actions and interventions are required to facilitate the health of a newborn. Very often the intervention required takes place long before the infant is born through maternal health education, prevention (family planning and antenatal care for example), vaccination, community participation, specific interventions like improved sanitation, and access to primary care near where a woman lives. Where the infant is delivered (at home, or in a facility), the quality of care received, breastfeeding, and the presence of community health workers also facilitate survival and health for the first year of life. These phases are referred to in the literature as

\footnotetext{
158 McKenzie and Ellis, "Community-Based Interventions to Improve Neonatal Survival in LowResource Settings," 197.

159 Reem M Ghandour et al., "Applying Collaborative Learning and Quality Improvement to Public Health: Lessons from the Collaborative Improvement and Innovation Network (CoIIN) to Reduce Infant Mortality," Maternal And Child Health Journal 21, no. 6 (June 2017): 1319, https://doi.org/10.1007/s10995-016-2235-2.
} 
"the continuum of care" as described by Darmstadt (2005) ${ }^{160}$ and it highlights a horizontal axis of life saving intervention points for a mother and infant. Multiple actions can take place over time to improve health outcomes for an infant. They can occur before conception (preconception), while the mother is pregnant (antenatal/prenatal), when the infant is born (delivery), and after the baby is delivered (postnatal) ${ }^{161162163}$. Let us now consider the other component of the model mentioned earlier in the chapter. The figure below represents the chronology of care phases in a woman's life where indirect interventions can mitigate infant mortality risks. This figure, in conjunction with the earlier bull's-eye figure, will be used throughout the paper to highlight various interventions that can shape IMR.

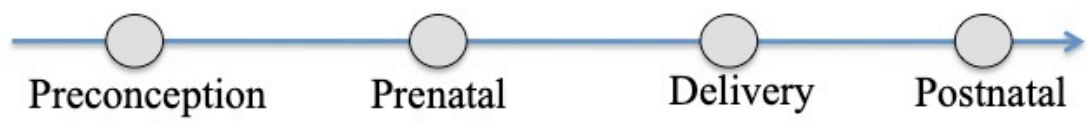

Figure 2: Chronology of potential care phases in a woman's life Ideally, there is a continuum of care and practical assistance to families and babies throughout the different phases of care for women in the childbearing years:

160 Gary L Darmstadt et al., “Evidence-Based, Cost-Effective Interventions: How Many Newborn Babies Can We Save?," The Lancet 365, no. 9463 (March 18, 2005): 977-88, https://doi.org/10.1016/S0140-6736(05)71088-6.

161 Darmstadt et al.

162 Bhutta et al., "Community-Based Interventions for Improving Perinatal and Neonatal Health Outcomes in Developing Countries."

163 Bhutta et al., "Reducing Maternal, Newborn, and Infant Mortality Globally." 
preconception, prenatal, delivery, and postnatal care. Developing countries, specifically those in crisis and low resource settings do not have the available finances, resources (quality care of doctors, nurses, health workers, facilities) or knowledge at specific intervention points to implement all aspects of the continuum of care as identified by health professionals. Sometimes, only a handful of interventions (like targeted tetanus vaccination programs for pregnant mothers, the importance of breastfeeding, or discussions about birth spacing from a health care provider before a mother leaves the maternity ward are implemented.

Below is an example, using the complete two-part figure, illustrating how both the indirect health mechanism as well as the indirect social and policy mechanisms intersect along the chronology of potential care phases during a woman's life. In the example below, the figure demonstrates a woman might have learned about the importance of breastfeeding after the delivery of her infant. In this case, breastfeeding and health education indirectly alleviated infant mortality in the postnatal phase.

For this example, we see that breastfeeding (an indirect health mechanism) alleviates infant mortality in the postnatal phase, and that health education (an indirect social and policy mechanism) also mitigates infant mortality during the postnatal period. 


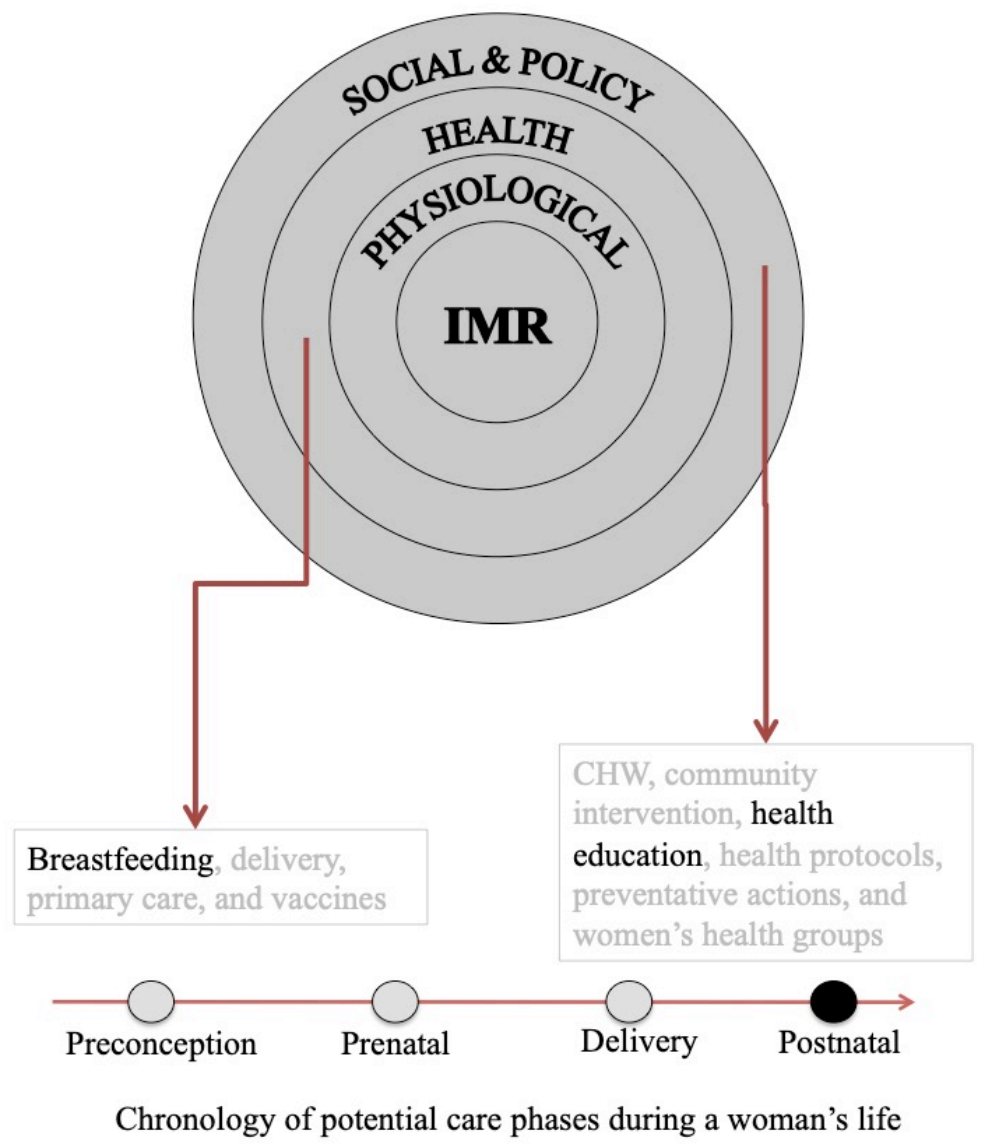

Figure 3: Intersection of the two indirect mechanisms that alleviate infant mortality with the chronology of potential care phases during a woman's life

Macro Structural Determinants

The primary determinant of a country's IMR level is its level of supportive structural conditions- the level of economic development, democracy, and governance. There is no mystery about why Belgium's IMR is lower than Botswana's. For the same reason, improvements in these levels generally predict improvements in the IMR. Rapid economic growth can generate new resources that reduce the IMR. The puzzle 
arises when the IMR declines rapidly in the absence of major improvements in levels of development, democracy, and governance.

So far, this paper has explored the literature that addresses the indirect mechanisms that can alleviate the proximate causes of IMR. We have essentially seen the micro positive steps that can be taken, at the individual level, to alleviate the proximate causes of infant mortality. The literature has been very specific and practical about the immediate impacts of certain interventions or practices that improve chances of infant survival. Now we turn our attention to considerations that explain the likelihood that both indirect mechanisms (health, and social and policy) are able to impact the proximate causes of infant mortality (i.e., that the concentric rings are influential). In the literature some scholars have focused generally on several macro structural factors as key determinants to development overall and mitigation of infant mortality in particular. On the whole, three macro structural factors emerge as important to IMR: democracy, governance and public spending, and economic growth. By in large, it is clear that IMR in developed western democracies is better than in countries with authoritarian leaders, poor governance and low public spending, and lagging economic growth. We know that countries with low IMR mainly demonstrate strong aspects of these three macro structural factors. However, there is not an overwhelming consensus in the literature about the relative and specific contributions of each to IMR reductions or in what order they must follow in developing countries. They are not rival hypotheses; instead they often hang together. 


\section{Democracy}

The macro structural role of democracy to specifically reduce IMR is inconclusive: on the one hand, it is thought to help and on the other, it appears to have no effect. Some researchers offer that democracy improves human development because it allows for citizen participation in government that in turn requires governments to be more responsive and accountable to citizens'164165166167168169170171. Navia and Zweifel (2000) show how in almost all cases IMR in democracies is lower than in dictatorships ${ }^{172}$. Other research denies the role of democracy in improving outcomes of the poor: "if democracy produces better outcomes for low-income families, then countries that transit from autocratic to democratic rule should see improvement in their infant and child mortality rates. In general, they do not"173. Ross (2006) concedes studies do demonstrate that democracies are more successful than other

\footnotetext{
164 Carles Boix, "Democracy, Development, and the Public Sector," American Journal of Political Science, 2001, 1-17.

165 David S. Brown and Wendy Hunter, "Democracy and Human Capital Formation: Education Spending in Latin America, 1980 to 1997," Comparative Political Studies 37, no. 7 (2004): 842-864. 166 David S. Brown and Ahmed Mushfiq Mobarak, "The Transforming Power of Democracy: Regime Type and the Distribution of Electricity," American Political Science Review 103, no. 2 (2009): 193213.

167 Hazem Adam Ghobarah, Paul Huth, and Bruce Russett, "Comparative Public Health: The Political Economy of Human Misery and Well-Being," International Studies Quarterly 48, no. 1 (2004): 73-94. 168 Masayuki Kudamatsu, "Has Democratization Reduced Infant Mortality in Sub-Saharan Africa? Evidence from Micro Data," Journal of the European Economic Association 10(6) (December 2012): 1294-1317.

169 David A. Lake and Matthew A. Baum, "The Invisible Hand of Democracy: Political Control and the Provision of Public Services," Comparative Political Studies 34, no. 6 (2001): 587-621.

170 Seymour Martin Lipset, "Some Social Requisites of Democracy: Economic Development and Political Legitimacy," American Political Science Review 53, no. 1 (1959): 69-105.

171 Edward N. Muller, "Democracy, Economic Development, and Income Inequality," American Sociological Review, 1988, 50-68.

172 Thomas D. Zweifel and Patricio Navia, “Democracy, Dictatorship, and Infant Mortality," Journal of Democracy 11, no. 2 (2000): 108.

173 Michael Ross, “Is Democracy Good for the Poor?," American Journal of Political Science 50, no. 4 (October 2006): 860.
} 
regime types at improving the lives of the poor but he argues many of these crossnational studies exclude successful nondemocratic countries due to missing or incomplete information ${ }^{174}$. If more of the nondemocratic countries were included in the cross-national studies (as well as correcting some "flaws"), then "democracy has little or no effect on infant and child mortality rates"175. The example of the Democratic People's Republic of Korea demonstrates that IMR declines are possible and significant (a 62\% decrease in IMR from 55.1 deaths per 1,000 to 20.7 per 1,000 in 2014) in undemocratic regimes, however liberal democratic South Korea, for the same time frame, had IMR at 4.5 and 3 respectively ${ }^{176}$. Ross (2006) does find that democracies can increase public funding for social programs but adds that the spending does not improve infant and child mortality rates ${ }^{177}$. Gerring et al. (2012) agree with Ross (2006) that there is not a strong relationship between "regime type" and IMR but there is a "robust causal relationship...if democracy is considered as a long run, historical phenomenon"178. Longevity of democracy appears to also impact other studies of developing countries. One study finds that democratization reduced infant mortality (almost immediately) in Sub-Saharan Africa as soon as multiparty elections took place that resulted in new leaders post- Cold War ${ }^{179}$. After democratization in the country, public health improvements appeared to be the

\footnotetext{
174 Ross, "Is Democracy Good for the Poor?"

175 Ross, 860.

176 Author's calculation

177 Ross, "Is Democracy Good for the Poor?," 862.

178 Gerring, Strom Thacker, and Alfaro, "Democracy and Human Development," 14.

179 Kudamatsu, "Has Democratization Reduced Infant Mortality in Sub-Saharan Africa? Evidence from Micro Data," 1315.
} 
important factor in reducing the infant mortality rate ${ }^{180}$. Another study of former

socialist countries in Central Asia and Eastern Europe reported an initial a drop in

life expectancy ${ }^{181}$ after the democratic shift but ultimately elements of freedom like

education and civil rights are consistent with income and longevity ${ }^{182}$. The literature

on democracy and IMR is mixed but on balance there is a demonstrated benefit to

population health.

\section{Governance and Public Spending}

Another macro structural consideration that scholars and practitioners

acknowledge as essential to IMR reduction is governance and public spending183 184

185186187188189190191192 193. Good governance, as opposed to poor governance, is

characterized by the following: "participation, rule of law, transparency,

\footnotetext{
180 Ibid., 1314.

181 Deaton, “Global Patterns of Income and Health," 3.

182 Deaton, 4.

183 Olusoji Adeyi et al., "Health Status during the Transition in Central and Eastern Europe: Development in Reverse?," Health Policy and Planning 12, no. 2 (January 1, 1997): 132-45, https://doi.org/10.1093/heapol/12.2.132.

184 Darmstadt et al., "Evidence-Based, Cost-Effective Interventions."

185 Deon Filmer and Lant Pritchett, "The Impact of Public Spending on Health: Does Money Matter?," Social Science \& Medicine 49, no. 10 (1999): 1309-1323.

186 Varun Gauri and Peyvand Khaleghian, "Immunization in Developing Countries: Its Political and Organizational Determinants," World Development 30, no. 12 (2002): 2109-2132.

187 Björn Halleröd et al., "Bad Governance and Poor Children: A Comparative Analysis of Government Efficiency and Severe Child Deprivation in 68 Low- and Middle-Income Countries," World

Development 48 (August 2013): 19-31, https://doi.org/10.1016/j.worlddev.2013.03.007.

188 Lawn et al., "Newborn Survival in Low Resource Settings-Are We Delivering?"

189 Andrew Sunil Rajkumar and Vinaya Swaroop, "Public Spending and Outcomes: Does Governance Matter?," Journal of Development Economics 86, no. 1 (2008): 96-111.

190 Ross, "Is Democracy Good for the Poor?"

191 Kunal Sen, “Governance and Development Outcomes in Asia,” Working Paper, ADB Economics Working Paper Series, No. 384 (Manila: Asian Development Bank (ADB), 2014).

192 Shalendra Sharma, "Democracy, Good Governance, and Economic Development," Taiwan Journal of Democracy 3, no. 1 (July 2007): 29-62.

193 Wise and Darmstadt, "Strategic Governance."
} 
responsiveness, consensus orientation, equity, effectiveness and efficiency, accountability and strategic vision"194. Good governance is easy to identify but hard to implement in some countries. It is especially important because it can determine the effectiveness of pubic spending. The effectiveness of health spending, for instance, on child mortality rates depends on adequate levels of governance: where governance is lacking, public health spending makes no impact ${ }^{195}$. One study demonstrates that public spending as a percent of GDP for public health lowers the under-5 mortality rate more significantly in countries with good governance, and it has no impact in countries with poor governance ${ }^{196}$. In some countries with poor governance, the positive impact of public spending on education is also reduced and public health expenditures can be lost entirely ${ }^{197}$. Whereas there are some examples of low income countries, in sub-Saharan Africa, that saw the highest impact of public spending and they were on target to achieve their Millennium Development Goals ${ }^{198}$. Hu and Mendoza (2013) find "the level of public social spending and the quality of governance matter for child health outcomes" but they are unsure how the two variables interact ${ }^{199}$. In this case, $\mathrm{Hu}$ and Mendoza measure governance as

\footnotetext{
194 “Governance for Sustainable Human Development," UNDP Report, 1997, 5.

195 Emanuele Baldacci et al., "Social Spending, Human Capital, and Growth in Developing Countries," World Development 36, no. 8 (2008): 1329.

196 Rajkumar and Swaroop, "Public Spending and Outcomes," 97.

197 Baldacci et al., "Social Spending, Human Capital, and Growth in Developing Countries,” 1336.

198 Baldacci et al., 1336.

199 Bingjie Hu and Ronald U. Mendoza, "Public Health Spending, Governance and Child Health Outcomes: Revisiting the Links," Journal of Human Development and Capabilities 14, no. 2 (May 1, 2013): 299, doi:10.1080/19452829.2013.765392.
} 
the "control of corruption and the quality of bureaucracy"200. An earlier study suggests that while medicine is effective, "the impact of public spending on health is much more complicated than the effectiveness of the services purchased"201. One reason for the complication has to do with the efficiency of public sector spending and health service delivery, also described as the "chain from public spending to health status"202. Delivery of effective care to mothers and their infants who need it the most is the real challenge ${ }^{203}$. The effectiveness of government varies "across time, regions, and countries" and it appears to "vary with the level of economic development of each country"204.

\section{Economic Growth}

The role of economic growth in development is almost axiomatic: "wealthier nations are healthier nations"205. Economic growth improves lives but not always the infant mortality rate according to some researchers. One study shows that there is a "positive cross-country correlation between health and income" but that there is "no cross-country correlation between reductions in infant and child mortality and rates of economic growth"206. Hanmer et al. (2003) show mortality in Africa decreased

\footnotetext{
$200 \mathrm{Hu}$ and Mendoza, 287.

201 Filmer and Pritchett, "The Impact of Public Spending on Health," 1320.

202 Filmer and Pritchett, 1320.

203 Lawn et al., "Newborn Survival in Low Resource Settings-Are We Delivering?," 57.

204 Soo-Young Lee and Andrew B. Whitford, "Government Effectiveness in Comparative Perspective," Journal of Comparative Policy Analysis: Research and Practice 11, no. 2 (June 1, 2009): 260, https://doi.org/10.1080/13876980902888111.

205 Lant Pritchett and Lawrence H. Summers, "Wealthier Is Healthier," The Journal of Human

Resources 31, no. 4 (1996): 841, https://doi.org/10.2307/146149.

206 Deaton, "Global Patterns of Income and Health," 22.
} 
"despite falling income, indicating that mortality decline can take place independent of income growth"207. Filmer et al. (1999) suggest that there is a large gap between public spending for improved health and the reality of health. They also find that "the differences across countries in infant and child mortality are well explained by economic and social factors" 208 instead of just public spending. Public spending on health within a country has shown a little impact 209 . For instance, one study finds that increases in health spending of " 1 percentage point of GDP is associated with an increase of 0.6 percentage point in the under- 5 child survival rate and a rise of 0.5 percentage point in annual per capita GDP growth" 210 . The level of economic development, or GDP is "an important factor underlying between-country differences in child health. This underscores its potential for improving levels of population health and agrees with ecological studies in developing countries" ${ }^{211}$. Another study agrees that as per capita income increases, infant mortality improves ${ }^{212}$. Other research supports the idea that public health spending reduces

\footnotetext{
${ }^{207}$ Lucia Hanmer, Robert Lensink, and Howard White, "Infant and Child Mortality in Developing Countries: Analysing the Data for Robust Determinants," The Journal of Development Studies 40, no. 1 (2003): 104.

208 Filmer and Pritchett, "The Impact of Public Spending on Health," 1310.

209 Filmer and Pritchett, 1309.

210 Baldacci et al., "Social Spending, Human Capital, and Growth in Developing Countries," 1336.

211 Michael H. Boyle et al., "The Influence of Economic Development Level, Household Wealth and Maternal Education on Child Health in the Developing World," Social Science \& Medicine 63, no. 8 (2006): 2250.

212 Pritchett and Summers, "Wealthier Is Healthier," 842.
} 
infant mortality through "a 1 percentage point increase in health care spending decreases infant and child mortality rates by about 3 per 1000 live births" ${ }^{\prime 213}$. So, studies vary widely in their conclusions regarding the role of democracy, governance and public spending, and economic growth in reducing IMR in developing countries. The literature does not say these three macro structural elements are impediments to improved IMR. No, on the contrary, these elements are frequently inseparable, and tangled in a dependent back and forth discussion of how development for most of the world's population can be achieved. The prominence of these macro structural elements for development and the improvement in IMR cannot be diminished even if it cannot be positively associated. One just has to look at conditions in the absence of democracy, governance and public spending, and economic growth and the results on IMR are objectively clear- more infants are at risk or die, and development is stunted. Would it be fair to say then that some developing countries experience a macro-negative scenario (i.e., the absence of three cornerstone elements of development) where structural pessimism permeates the government? This leaves us to consider what happens in the real world where IMR improvements are made in developing countries regardless of structural pessimism. Are there micro-positive mechanisms at work pushing back against the oppressive, downward thrust of structural dysfunction?

\footnotetext{
${ }^{213}$ Sanjeev Gupta, Marijn Verhoeven, and Erwin R. Tiongson, "The Effectiveness of Government Spending on Education and Health Care in Developing and Transition Economies," European Journal of Political Economy 18, no. 4 (2002): 732.
} 
Macro-negative (structural pessimism) vs. Micro-positive (agency optimism)

This study inquires how the IMR improves when democracy, good governance and public spending, and economic growth are unavailable in developing countries. For instance, democracy, good governance and public spending, and economic growth are often impossible to achieve with political instability and civil conflict that are often present in such developing country contexts. This begs the question of how strategic pockets of governance can be created when the "highest levels of neonatal mortality around the world are, for the most part, characterized by weak governance and chronic, intermittent political violence"214. For the most part, poor countries are characterized by substandard structural or national conditions that cause undesirable maternal and infant health outcomes. For example, if a country is involved in a civil war this condition might be considered macro-pessimistic or a "gloomy vision" 215 because the big picture for the health of the country is not positive, on the contrary it is negative. The structure of the government, the quality of the bureaucracy and the strength of economic growth are also conditions that, if negative, can be considered macro-negative. Macro-negative represents scenarios at the national level that are considered incompatible with positive outcomes. A civil war raging in a country is antithetical to longevity. This example makes a point that national (or structural level) conditions can obviously jeopardize population health. Other ways to identify macro-pessimism is to call it

214 Wise and Darmstadt, "Strategic Governance," 391.

215 Albert O. Hirschman, A Bias for Hope: Essays on Development and Latin America (New Haven: Yale University Press, 1971), 354. 
structural determinism, i.e., the structure determines potential or capacity. Also known as structural constraint, i.e., the structure constrains outcomes or options ${ }^{216}$. This situation can occur in developing as well as developed nations. When there is "macro-negative" information it frequently describes the "causes and consequences of policies, usually by employing aggregate data to show why policies do not work"217. At the national or structural level if the economy, regime, or bureaucracy are not optimal it is probably a result of poor policy. For example it might be that democracy is faltering (or non-existent) due to questions of legitimacy, governance is poor due to corruption, and growth is faltering due to limited industrial capacity. However, the structure does not always determine the outcome for the individual. Macro-negative conditions are not fate because there is individual human agency that can circumvent or pivot around negative structural roadblocks. When the micro level (as opposed to the macro level) functions well it can be an affirmation of adept agency. Micro-positive (also referred to as agency optimism) occurs when something at the local or individual level functions well. Micro-positive conditions can take place even when macro conditions are poor. When there is "micro-positive" information it is typically "positive" because it reveals at local levels "what policies and programs do work under specified conditions"218219.

\footnotetext{
${ }^{216}$ John Lonsdale, "Editorial: Agency in Tight Corners: Narrative and Initiative in African History," Journal of African Cultural Studies 13, no. 1 (2000): 7.

217 William N. Dunn, Public Policy Analysis, 5 edition (Boston: Routledge, 2011), 12.

218 Dunn, 12.

219 Walter Williams, Social Policy Research and Analysis: The Experience in the Federal Social Agencies (American Elsevier Publishing Company, 1971), 8.
} 
A term used to capture the contradiction of both macro-negative and micro-positive conditions at the same time is called the "macro-micro paradox". The term "macromicro paradox" was used initially to describe the benefit of international aid on developing countries at the micro level even without evidence necessarily of the same at the macro level220221. The paradox is that confidence is seen, felt, or measured at micro levels but macro measurement cannot capture the same optimism. For instance, the effectiveness of aid in Kenya was deceptive in one study (Mosley, 1986). The growth of the economy did not show any appreciable change due to donor funding but World Bank projects' returns were "respectable"222. Mosley concluded, "there is a sharp discrepancy between macro- and micro- level measures of the effectiveness of overseas aid...the pessimistic macro-results must be interpreted with extreme caution"223.

The macro-micro paradox was subsequently explored in a study of agriculture and land management in Sub-Saharan Africa ${ }^{224}$. Macro-level pessimism consists, in this instance, as regional food insecurity not expected to improve before 2030 and environmental degradation including water scarcity and land clearance ${ }^{225}$. The

\footnotetext{
220 Mark McGillivray et al., “Controversies over the Impact of Development Aid: It Works; It Doesn't; It Can, but That Depends...," Journal of International Development: The Journal of the Development Studies Association 18, no. 7 (2006): 1032.

221 Paul Mosley, Overseas Aid: Its Defence and Reform. (Wheatsheaf Books, 1987).

222 Paul Mosley, “Aid-Effectiveness: The Micro-Macro Paradox,” Ids Bulletin 17, no. 2 (1986): 25.

223 Mosley, 26.

${ }^{224}$ C. P. Reij and E. M. A. Smaling, "Analyzing Successes in Agriculture and Land Management in SubSaharan Africa: Is Macro-Level Gloom Obscuring Positive Micro-Level Change?," Land Use Policy 25, no. 3 (2008): 410-420, https://doi.org/10.1016/j.landusepol.2007.10.001.

225 Reij and Smaling, "Analyzing Successes in Agriculture and Land Management in Sub-Saharan Africa," 410.
} 
aggregate data was measured by regional FAO statistics for projected fertilizer use, trends in crop yield, and UNEP reports ${ }^{226}$. The aggregate data suggest gloom, pessimism, and/or constraint on the region. But, what can be said for the smaller case studies in SSA to the contrary? The smaller, positive cases demonstrating success "in crop, livestock and forest production, in environmental management, in empowerment and capacity building"227 are frequently lost in favor of "big" studies. An illustration comes from Niger where in 2005 international news reported widespread drought and hunger however what was not accentuated were farmers using sustainable techniques for over two decades and as a consequence there was significant "re-greening". Many have attributed the "natural generation on their cultivated fields" to saving lives because they had trees to sell as "firewood" and "leaves were harvested, consumed or sold" during hard times ${ }^{228229}$. So, the macropessimism at the country level belied instances of micro-optimism. The SSA study finds that there is success at the micro-level described by Haggblade (2004) ${ }^{230}$ as " $a$ significant, durable change in agriculture resulting in an increase in agriculturally derived aggregate income, together with reduced poverty and/or improved

\footnotetext{
${ }^{226}$ Reij and Smaling, 410-11.

227 Reij and Smaling, 410.

228 Reij and Smaling, 411.

${ }^{229}$ M. Larwanou, M. Abdoulaye, and C. Reij, "Etude de La Régénération Naturelle Assistée Dans La Région de Zinder (Niger)," Washington, DC: International Resources Group, 2006.

230 Steven Haggblade, "Successes in African Agriculture: Building for the Future," in Findings of an International Conference, Pretoria, South Africa.(December 1-3, 2003). InWent, IFPRI, NEPAD, CTA, 2004.
} 
environmental quality"231. The successes experienced in agriculture and land management in SSA (e.g., higher yielding crops, improved water tables, decreased rural to urban migration, rural poverty reduction etc.) present a micro positive scenario $^{232}$. Those same successes are often not observed at national and international levels for a myriad of reasons: 1) population growth outweighs the advances, 2) errors in data, 3) local surpluses are not captured by statistics, and 4) local produce is not in demand ${ }^{233}$. This example demonstrates a macro-negative (structural pessimism) condition in SSA with a micro-positive (agency optimism) outcome.

If there are cases of countries where macro-structural conditions are poor, yet IMR is improving (and others where it is improving but not measured) it suggests the possibility of micro-positive factors that facilitate the provision of indirect mechanisms that alleviate the proximate physiological mechanisms of infant mortality. The micro-positive outcomes could be the result of different policy tools creating conditions that enable indirect health, and social and policy mechanisms to alleviate the proximate causes of infant mortality that ultimately improve local conditions.

This possibility can be illustrated by considering the three-ring model introduced earlier. However now we layer on two additional concentric circles beyond the

\footnotetext{
${ }^{231}$ Reij and Smaling, "Analyzing Successes in Agriculture and Land Management in Sub-Saharan Africa," 412.

232 Reij and Smaling, 414.

${ }^{233}$ Reij and Smaling, 414-19.
} 
indirect social and policy ring: an outer, exterior macro structural layer and an intervening strategic governance layer. The intervening strategic governance layer might act as a buffer between macro-structural pessimism and the micro indirect mechanisms that alleviate the physiological mechanisms of infant mortality, consistent with studies cited above.

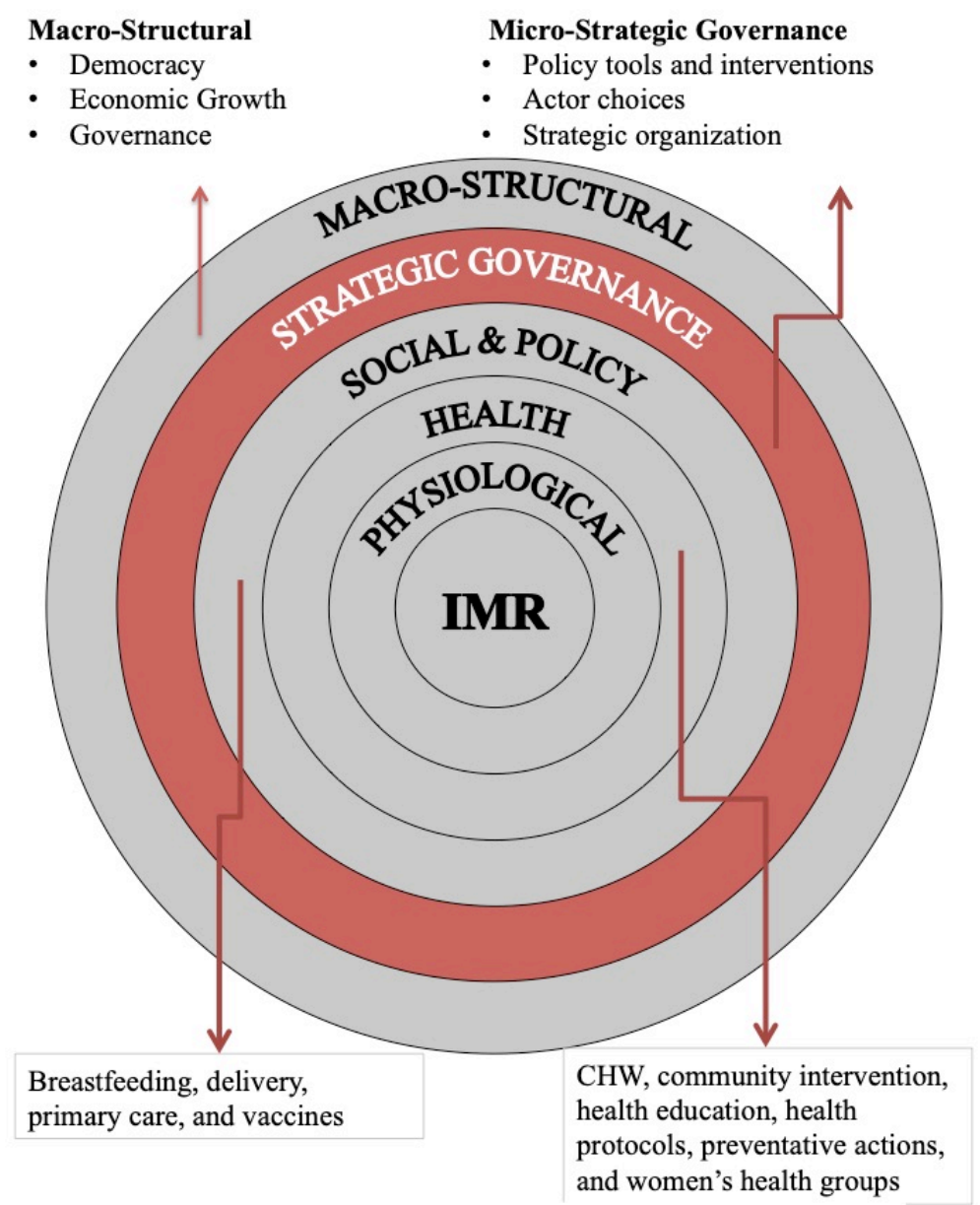

Chronology of potential care phases during a woman's life

Figure 4: Potential determinants of IMR model 


\section{Research Question}

What explains declines in IMR where economic growth, governance, and democratic accountability are weak? In particular, what "strategic governance" practices, if any, can be identified as contributing to the IMR decline in such cases? 


\section{Chapter 3: Research Design and Case Selection}

Research Paradigm

Now that we have thought about existing literature and theories relating to IMR improvement we face the question of how to develop a valid research design to study the IMR reduction in crisis conditions. Generally speaking, an inductive or deductive approach is taken in order to answer a research question. Inductive research begins with an observation to which additional observations are added, building from the bottom up. The collected observations can reveal patterns. Repetitive patterns within the observations can suggest a hypothesis. A hypothesis, routinely observed can suggest a theory or social regularity ${ }^{234}$. Deductive research (also referred to as a hypothesis-testing approach) starts in the opposite direction of inductive research. The deductive approach to theory building begins with an idea or theory (at the top) and then works downwards checking to see if observations support the theory 235 .

One way to study improvements in IMR inductively would be to look for patterns in large quantitative data sets. A traditional quantitative, or empirical analysis of IMR might lead one to conclude that macro level influencers such as stability or form of government would directly improve IMR. A recent article on poverty in Mozambique suggests two different inductive (quantitative and qualitative) styles

\footnotetext{
234 Masami Nishishiba, Research Methods and Statistics for Public and Nonprofit Administrators: A Practical Guide, SAGE Research Methods Books \& Reference (Los Angeles: SAGE, 2014). 235 Nishishiba, 20-21.
} 
as a way to approach research but they are best "leveraged" on their own merit rather than combined because each delivers a different answer ${ }^{236}$. The social ontology of reality (i.e., what there is), coupled with epistemology (how we come to know it) helps shape our understanding of the world. How ontology and epistemology are combined in a research plan shape reality at a particular nexus; "different disciplines do not simply see the same thing (i.e., poverty) from different angles; rather, they see quite different things, and that the forms of knowledge they generate are distinct"237. For instance, understanding can be based on an emic (within a social group) or etic (from outside) experience so the specific type of information included shapes the answer ${ }^{238}$. It would be wrong to throw one measure out in favor of the other because different disciplines (like political science, public affairs and the physical sciences) each reveal different information. The approach this paper takes is to explore how the micro level qualitative practices can and do counteract the presumed impacts of macro level policies through analytic narratives.

Research into the causes of infant mortality rate declines can likewise proceed from an inductive or deductive direction. Reflecting the practical orientation of the subject, much research has taken the inductive approach, either using large-scale quantitative datasets or carefully observed patterns at the individual or group level.

\footnotetext{
236 Sam Jones and Inge Tvedten, "What Does It Mean to Be Poor? Investigating the QualitativeQuantitative Divide in Mozambique,” World Development 117 (2019): 153-166.

237 Jones and Tvedten, 164.

238 Jones and Tvedten, 153.
} 
A deductive approach, by contrast, would dedicate greater efforts to the formulation of conceptual categories and to the stipulation of logical conditions along with their ceteris paribus requirements for the reduction in infant mortality rates. This would then be coupled with the testing of those propositions and the discovery of additional ceteris paribus conditions leading to either a rejection, acceptance, or restatement of the initial theory.

What is the essential ontology (nature of social reality) of infant mortality? It involves both a physical reality (often discovered through deductive approaches), as well as a social and cultural one (often discovered through inductive approaches). Therefore, the research paradigm adopted here will be one that borrows from both approaches. In the first instance, large-scale quantitative data will be used to sort a large number of country case studies. The purpose is to apply the law-like generalizations about macro-structural causes of IMR to identify cases that appear to violate, or be inconsistent, with those law-like generalizations. Having used deductive and quantitative approaches for sorting, the research then moves to a selection of those cases for inductive and qualitative analysis. The specific technique adopted for those case studies will be the analytic narrative.

\section{Case Studies}

To begin, a Large $\mathrm{N}$ is used as the sorting mechanism, and then within those cases we sort again for the small $\mathrm{N}$. The small $\mathrm{N}$ design uses an inductive analytic narrative with both temporal and functional theories. Therefore, this research design uses multiple methods. Small-n study is selected using the "Most Different 
Systems" Design ${ }^{239}$ (MDSD). The MDSD looks at different cases with similar outcomes, to eliminate "factors differentiating social systems by formulating statements that are valid regardless of the systems within which observations are made"240.

A structured comparative (multiple) case study is then used with embedded multiple units of analysis ${ }^{241}$ to investigate the case countries. A case study can help illuminate decisions as well as institutions and actors that are instrumental in creating better outcomes ${ }^{242}$ within case countries. Multiple case studies also offer opportunities for replication which have the ability to "predict similar results or to predict contrasting results but for predictable reasons"243; thereby strengthening "support for an "initial set of propositions" and making a "compelling...overall study"244. Using an inductive, analytic narratives ${ }^{245}$ approach, within a processtracing framework ${ }^{246}$ the objective is to "reconstruct the meaning or significance of social arrangements and practices" 247 to lend an interpretation of how certain

\footnotetext{
${ }^{239}$ Henry Teune and Adam Przeworski, The Logic of Comparative Social Inquiry (New York: WileyInterscience, 1970).

240 Teune and Przeworski, 39.

${ }^{241}$ Robert K. Yin, Case Study Research: Design and Methods, 3rd Edition, 3rd edition (Thousand Oaks, Calif: SAGE Publications, Inc, 2002), 40.

242 Robert K. Yin, Case Study Research: Design and Methods, $3^{\text {rd }}$ Edition (Thousand Oaks, CA: SAGE Publications, Inc, 2002), 12-13.

243 Yin, 47.

244 Yin, 46.

245 Robert H. Bates et al., Analytic Narratives, 1st US Edition 1st Printing edition (Princeton, N.J: Princeton University Press, 1998).

246 David Collier, “Understanding Process Tracing," PS: Political Science \& Politics 44, no. 04 (October 2011): 823-30, https://doi.org/10.1017/S1049096511001429.

247 Daniel Little, Varieties of Social Explanation: An Introduction to the Philosophy of Social Science (Westview Press, 1991), 68.
} 
conditions are achieved. The process tracing helps identify micro-foundations versus mechanisms that spur IMR improvement. A comparative case study looks at two or more cases where "specific phenomena"248 are examined to find similarities, differences and/or patterns throughout all of the cases, which in turn can help develop a theory ${ }^{249}$. The comparative (multiple) case study aims for "thick description" 250 with the goal of focusing on a few variables for deeper analysis that can contribute to theory building 251 but not so thick as to make comparisons useless due to cultural contexts ${ }^{252}$.

Analytic narrative

The analytic narrative approach to case study often looks at interactions between politics, economics and the role of institutions ${ }^{253}$. The case studies will be narrative (paying "close attention to stories, accounts, and context"), and analytic by "extract[ing] explicit and formal lines of reasoning"254, i.e. it is a case study with "an underlying model that motivates analysis and frames the empirical materials"255. Using documents, archives and secondary literature to identify “actors' preferences, their perceptions, their evaluation of alternatives, the information they possess, the

\footnotetext{
248 Shelagh Campbell, "Comparative Case Study," in Encyclopedia of Case Study Reserach (Thousand Oaks: SAGE Publications, Inc, 2012), 175.

249 Campbell, 175.

250 Clifford Geertz, "Thick Description: Toward an Interpretive Theory of Culture," Readings in the Philosophy of Social Science, 1994, 1.

251 Campbell, "Comparative Case Study," 176.

252 Geertz, "Thick Description."

253 Bates et al., Analytic Narratives, 10.

254 Bates et al., 10.

255 Kenneth A. Shepsle, "Rational Choice Institutionalism," The Oxford Handbook of Political Institutions, $2006,13$.
} 
expectations they form, the strategies they adopt, and the constraints that limit their actions" 256 in order to "piece together the story that accounts for the outcome of interest: 257 decreased infant mortality rates in countries with poor quality governance. A narrative is first formed for each of the select case countries, then "modeling the processes that produce the outcomes"258 begins using studies demonstrated to improve infant mortality in developing countries to describe or explain what has occurred in that particular instance.

Select surveys can provide consistency or an "iterative analysis" 259 cross-nationally while a "post hoc" 260 comparison of the survey questions responses can potentially reveal how some countries with poor governance are able to decrease the IMR beyond the average decrease. Looking "holistically at how a particular action fits into a vast network of beliefs and practices"261 in the comparative case studies can illuminate understanding of how such counter intuitive conditions resulted. While not conclusive, an interpretive, inductive approach is an attempt to explain the paradox of decreasing IMRs in countries with poor governance (which is contrary to much literature).

The comparative case study is also useful to study change over time that might lend additional description. A drawback of the comparative case study is that it is not "a

\footnotetext{
256 Bates et al., Analytic Narratives, 11.

257 Bates et al., 11.

258 Bates et al., 12.

259 Campbell, “Comparative Case Study,” 175.

260 Campbell, 175.

261 Todd Jones, "Interpretive Social Science and the" Native's Point of View": A Closer Look,"

Philosophy of the Social Sciences 28, no. 1 (1998): 37.
} 
truly experiment research design, with a randomized treatment"262 rather it is selective with a potentially arbitrary set of criteria, i.e., the voice and accountability indicator is a proxy for democracy and the government effectiveness indicator is a proxy for governance. While not empirical, or universal, the comparative case study does provide an opportunity to interpret a unique set of conditions to potentially help understand how the IMR decreased at a greater rate in some developing countries than others.

\section{Case selection}

This study looks for purposive cases where there is both a crisis of democracy and governance (as measured by a Worldwide Governance Indicators from the World Bank) as well as slow economic growth, and a better than average decline in IMR. The case selection uses an XY scatterplot to identify countries that over perform the average decrease in the IMR for 106 developing countries and underperform in democracy and governance based on the average of two governance indicators. By selecting cases with poor governance and improved infant mortality rates, the countries can be examined through comparative case study to identify conditions that might describe the provision for improvement. Therefore, the case selection process is looking for countries that "over performed" (i.e., above average decrease in IMR) in reducing their IMR despite poor governance as well as low economic

\footnotetext{
262 John Gerring et al., "Democracy, History, and Economic Performance: A Case-Study Approach," World Development 39, no. 10 (October 2011): 1736, https://doi.org/10.1016/j.worlddev.2011.04.029.
} 
growth. This intentional "selection on the dependent and independent variables" can then be used to generate hypotheses.

Democracy and governance are measured using the voice and accountability, and the government effectiveness indicators from the Worldwide Governance Indicators of the World Bank to rank 106 developing countries. The voice and accountability indicator measures how democratic a country is while the government effectiveness indicators measure level of governance. The simple average of these two scores is plotted on the X-axis for each country from data points (ten from each of the two indicators) from 1996 to 2014, in two year intervals: 1996, 1998, 2000, 2002, 2004, $2006,2008,2010,2012$, and 2014 . The data is taken in two year intervals because the data was sporadic in initial reporting by the World Bank, i.e., reporting initially in 1996,1998 , and 2000, then every subsequent year there after. The indicators have a score range from -2.50 to 2.50 . For this study, the scoring range is broken up into three categories: crisis, moderate, and stable. Countries in the crisis category have an average score from -2.5 to -0.85 . Countries in the moderate category have an average score from -0.84 to .83 , and countries in the stable category have an average score from .84 to 2.5 .

Next, the IMR is plotted on the Y-axis. It is calculated with data from 1996 to 2014, by taking the log of the percent difference between 2014 and 1996. The average logarithmic transformation of the percent change in the IMR in the developing 
country dataset from 1996 to 2014 is $1.66^{263}$. For case selection, a country must have a score over the average 1.66 IMR and a score below -0.84 for the governance indicator. See Appendix A.

Fifteen countries from the dataset of developing countries (see appendix for all countries in the dataset) fit into this category of poor governance measures and above average decrease in the IMR: Azerbaijan, Belarus, Cambodia, Republic of the Congo, Eritrea, Ethiopia, Guinea, Iran, Korea (Democratic People's Republic of), Liberia, Libya, Rwanda, Syrian Arab Republic, Tajikistan, and Yemen. These fifteen countries are possible cases to investigate.

Variable 1 (x axis): The average of two Worldwide Governance Indicators: Voice and Accountability, and Government Effectiveness indicators from 1996 to 2014 in two-year increments.

Variable 2 (y axis): Decrease in the IMR from 1996 to 2014.

See Appendix A

263 Author's calculation from World Bank data. 


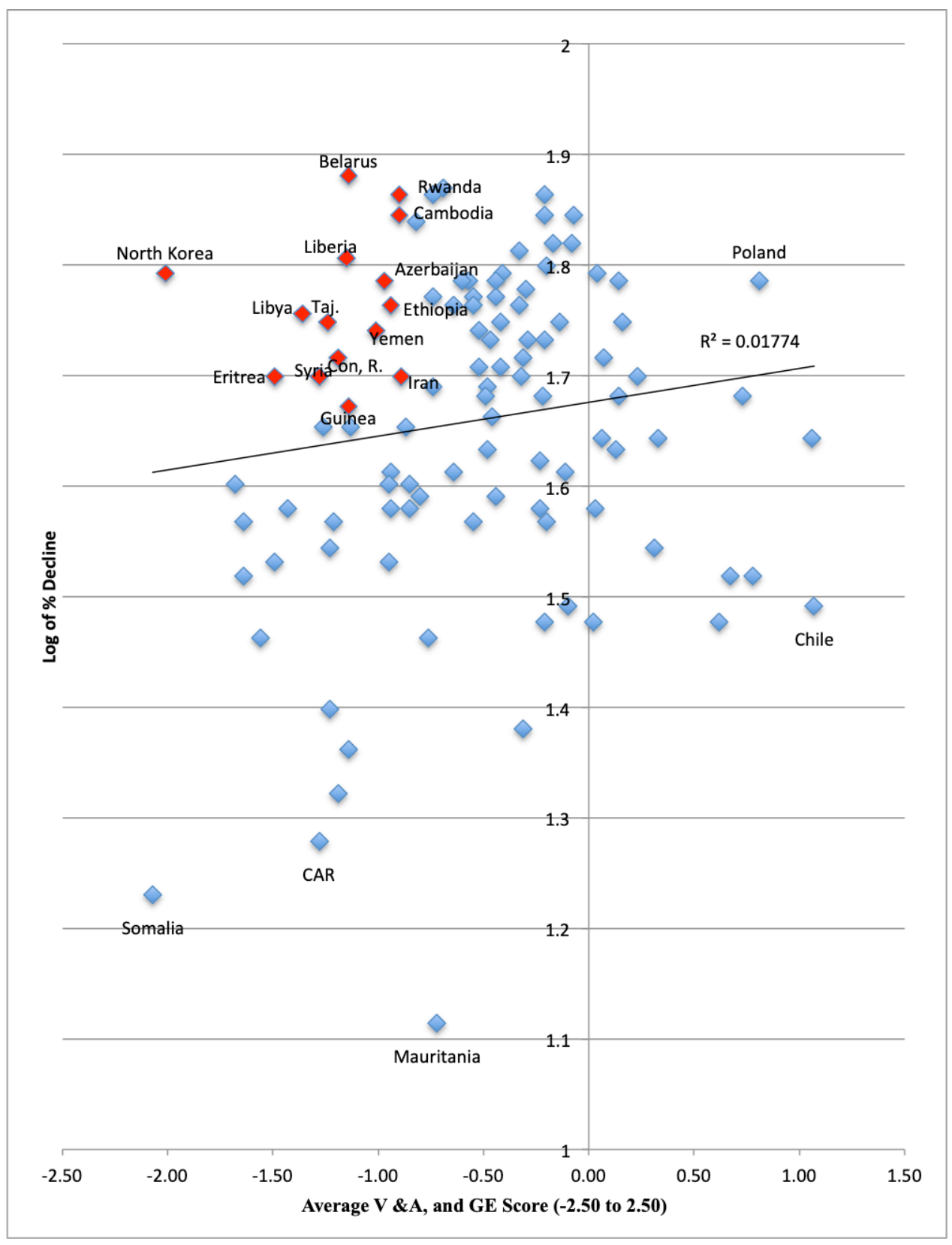

Figure 5: 106 developing countries as measured by average governance scores and the log of the percent decrease in IMR rates.

The fifteen countries highlighted in the upper left quadrant in figure 5 (i.e., Azerbaijan, Belarus, Cambodia, Republic of the Congo, Eritrea, Ethiopia, Guinea, Iran, Korea Democratic People’s Republic, Liberia, Libya, Rwanda, Syria, Tajikistan, and 
Yemen) represent countries that have above average improvement in the infant mortality rate and have CRISIS category governance scores. These potential case study countries have IMR with more than average decreases of $48 \%$.

From the fifteen countries an additional measure is considered: average GDP growth (annual percentage). The average GDP growth from 1996 to 2014 is $4.77 \%$ for 106 countries in the study dataset ${ }^{264}$. Azerbaijan, Belarus, Cambodia, Ethiopia, Liberia, Rwanda, Syria, Tajikistan all had economic growth greater than 4.77\% from 1996 to 2014 so they will be excluded from the case country selection dataset ${ }^{265}$. They are excluded because the better than average economic growth might impact the IMR. The countries have economies that grew too fast during the study time frame and the stronger than average growth is not consistent with the crisis countries this study is selecting for. Korea, Democratic People's Republic, and Ethiopia are excluded as well due to lack of information for the specified time frame. The revised dataset includes: Republic of the Congo, Eritrea, Guinea, Iran, Libya, Syria and Yemen.

This research focuses on two countries Republic of the Congo (ROC) and Republic of Yemen (ROY) selected by employing the "Most Different Systems" Design"266 from a revised dataset. ROC and ROY have different geographies, religions, histories (one colonial, the other partly so), and culture. The task is to identify if there are

\footnotetext{
${ }^{264}$ Author's calculation from The World Bank, “GDP Growth (Annual \%),” World Bank national accounts data, and OECD National Accounts data files, 2017, https://data.worldbank.org/indicator/NY.GDP.MKTP.KD.ZG?view=chart. 265 The World Bank.

266 Teune and Przeworski, The Logic of Comparative Social Inquiry.
} 
intentional mechanisms within the two case study countries that facilitate a greater than average decrease in IMR while governance is still poor and economic growth is underwhelming.

Looking at the two case study countries using longitudinal data, intentional mechanisms of strategic governance might be revealed. They will most likely be context specific, but there may be overlap between successful strategies used to improve the IMR.

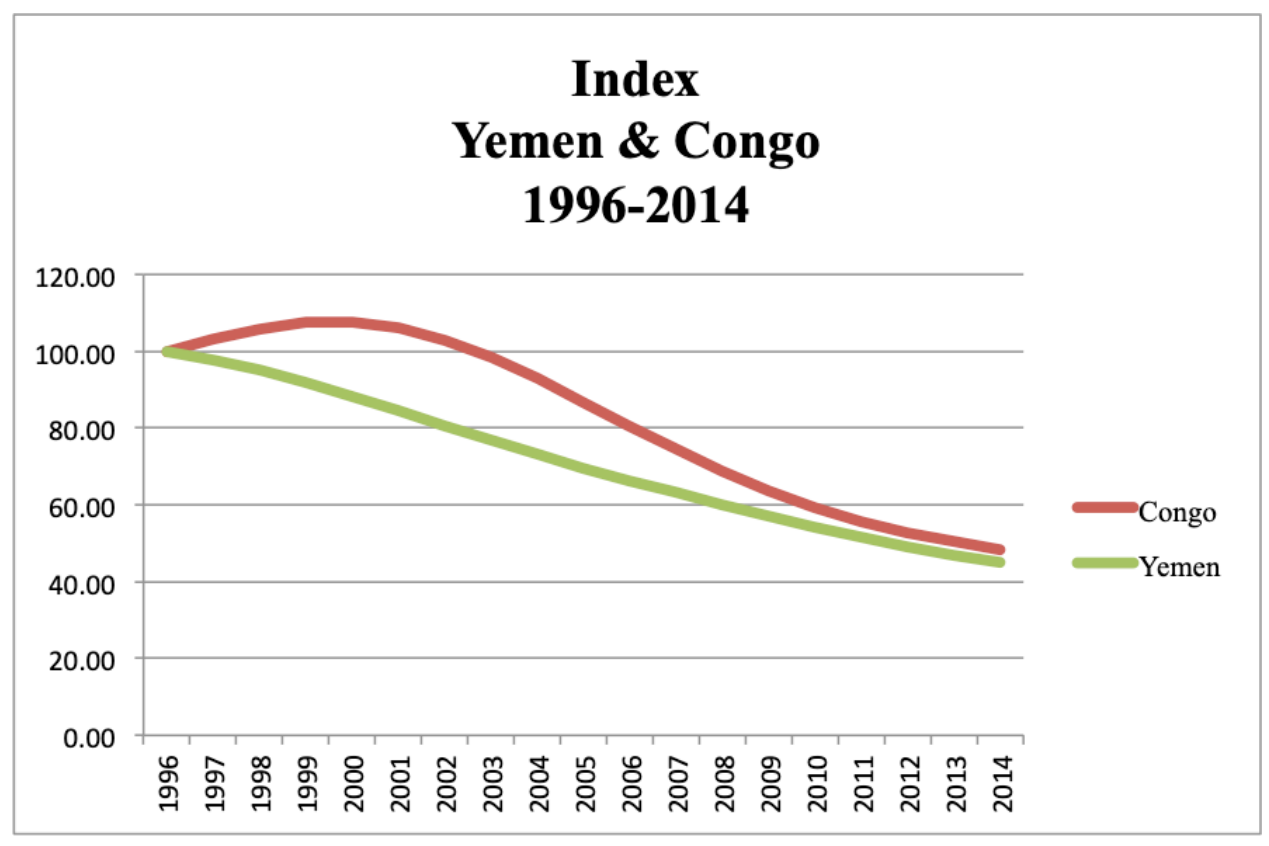

Figure 6: IMR decline in ROC and ROY from 1996 to 2014

To begin, data collected from existing data sets for the Republic of the Congo and Yemen is compared to discover what indirect mechanisms that alleviate the proximate physiological factors of infant mortality changed over time as a starting point to explore how the IMR in each country changed. Initial longitudinal data 
comes from the Demographic and Health Surveys (DHS) from USAID, the Multiple Indicator Cluster Surveys (MICS) from UNICEF, and UNESCO Institute for Statistics (UIS) for each country that is compared longitudinally. The available country datasets do not necessarily align in time for the two selected countries but they are constrained within 1996-2014 frame of the overall study. In Congo, Rep. data from the first available DHS for that country in 2005 and it is compared with data from the 2011-2012 DHS; whereas Yemen DHS data comes from both 1997 and 2013. Some indirect mechanisms that alleviate the proximate mechanisms of infant mortality improvement are revealed through data presented in the Demographic Health Surveys and they might reveal governance practices that impact the greater than average change in the IMR: age at first birth, age of mother at birth for infant mortality, birth intervals, IMR based on mother's age at birth, source of antenatal care (ANC), number of ANC visits, mother's level of education and resulting IMR, knowledge of HIV prevention, knowledge of oral rehydration salts (ORS), vaccinated before age 12 months, postnatal exams for women who gave birth by days, postnatal exams for women who did not give birth in a health facility by days, household characteristics, household possessions, women exposed to media, percent of women who have heard a family planning message in radio, $\mathrm{TV}$, journal /magazine, or poster/flyer. 


\section{Data sources}

The DHS Program began in 1984 and currently works in 93 of countries. DHS has conducted over 70 nationally representative surveys in more than 50 countries ${ }^{267}$. The DHS surveys are "nationally-representative household surveys [both a household schedule and an individual questionnaire are used] that provide data for a wide range of monitoring and impact evaluation indicators in the areas of population, health and nutrition"268. The DHS typically includes large samples between 5,000 and 30,000 households and they are conducted over time, often in 3 to 5-year increments to demonstrate change within countries ${ }^{269}$. The household schedule provides basic demographic information about family members and is used to identify woman who are of reproductive age. The women identified in their reproductive years are then given an individual survey to ask questions about the health of their children, reproductive history, knowledge of contraception and its use, and preferences for fertility. Standardization of surveys is assisted through the use of "model questionnaires, manuals, field procedures, and through the technical support provided by DHS staff"270. The DHS sample should, typically, cover 100

\footnotetext{
267 Macro International Incorporated, “Sampling Manual” (Calverton, Maryland, 1996), https://www.google.com/search?safe=off\&client=safari\&rls=en\&ei=4j30X07wJuq00gKg6LLAAw\&q =sampling+manual+macro+international+incorpoarted+1996\&oq=sampling+manual+macro+intern ational+incorpoarted+1996\&gs_l=psy-ab.3..0i71l2 $.0 .0 . .4745 \ldots 0.0 . .0 .0 .0 \ldots \ldots . .0 \ldots . . . g w s-$ wiz.7A5bE0jby2U.

268 "The DHS Program - Demographic and Health Survey (DHS)," accessed June 2, 2019, https://dhsprogram.com/What-We-Do/Survey-Types/DHS.cfm.

269 "The DHS Program - Demographic and Health Survey (DHS)."

270 Macro International Incorporated, "Sampling Manual."
} 
percent of a country's population except in extreme circumstances of war, or in cases where the population is too widely distributed 271 .

\section{Research Strategy}

The research will thus proceed in the following fashion: first, the country background of each case country will be highlighted to include pre-colonial, colonial, Cold War, and post-Cold War histories to provide an overall context for the case study of IMR improvement. Next, the macro-structural weaknesses (democracy, governance, and economic growth) will be described to reveal the broadly speaking, negative conditions in during which infant morality improved. The following section details the decline in IMR, after which the indirect mechanisms that alleviate the proximate causes of infant mortality are explored in relation to the chronology of care for women. Comparable questions from longitudinal Demographic Health Surveys (DHS) data from each country will be utilized to identify chronology of care IMR improvements. Discussed next is the case study process tracing and analytic narrative development that considers institutional drivers that impact the indirect mechanisms that alleviate the proximate causes of infant mortality. Finally, an initial statement of strategic governance findings for is made for each case country.

\section{Chapter 4: Republic of Congo}

This research will thus proceed in the following fashion: a) country background of pre-colonial, colonial, Cold War and post-Cold War history, especially as relevant to

\footnotetext{
271 Macro International Incorporated, 1.
} 
IMR; b) description of macro-structural weaknesses of democracy, governance, and economic growth; c) a description of IMR decline is described using surveys from two different time periods; d) description of indirect mechanisms that alleviate the proximate causes of infant mortality; e) case study analytic narrative development and analyzing the narrative; f) initial statement of "strategic governance".

a. Country background

Pre-colonial

Long before the slave trade, the colonists, and the dictators, the region of current day Congo was characterized by "equality, individual autonomy, and social commitment to the welfare of others"272. Pygmies who were then gradually replaced by the Bantus originally populated this western equatorial Africa region. The Bantu tribes were organized into roughly three states when explorers arrived in the fifteenth century: north of the Congo River was the Kingdom of Loango (part of current day Gabon and ROC); Congo or Makongo, stretching south to the Benguela River (in the middle of current day Angola); and Anzil, inland around the Niari River (whose headwaters begin around Brazzaville) ${ }^{273}$. From ancient times until the emergence of the kingdoms, the social organization of people was primarily established around a "big man" who headed a household constituted by ten to forty

\footnotetext{
272 John Frank Clark, The Failure of Democracy in the Republic of Congo (Boulder, Colo.: Lynne Rienner Publishers, 2008), 47.

273 Great Britain. Naval Intelligence Division, French Equatorial Africa \& Cameroons, Great Britain. Naval Intelligence Division. Geographical Handbook Series (London, 1942), 228.
} 
people that over time, organized into villages, chiefdoms, principalities, and finally kingdoms ${ }^{274275}$. The rulers were the ultimate authority and society was marked by different classes, with elites responsible for the emergence of "indigenous slavery" in the area ${ }^{276}$. Women and indigenous groups were considered inferior and marginalization continues to this day. 277 Portuguese explorers were the first Europeans of record to discover the West African coast in the late fifteenth century. Lopez Gonsalvez and Fernando Vaz, under the rule of King Alfonso V, found and named both an island and a cape after themselves (Cape Lopez, and Fernan Vaz) on the coast of Gabon ${ }^{278}$. Another Portuguese explorer, Diego Cam, went further south and "discovered" the mouth of the Congo River in $1484^{279}$. Cam named the river Zaire and generally claimed the Portuguese had arrived by "inscribing the royal arms of Portugal on the cliffs" 280 . Not long after, Portuguese missions worked in West Africa ${ }^{281}$, and by the mid sixteenth century the Dutch, British and French ${ }^{282}$ started trading powder, weapons, tobacco, spirits, beads, silk and woven items for gold-dust, ivory, palm-products and

\footnotetext{
274 Jan Vansina, Paths in the Rainforests: Toward a History of Political Tradition in Equatorial Africa (Madison, Wis.: University of Wisconsin Press, 1990), 74, 147, 152-58.

275 Clark, The Failure of Democracy in the Republic of Congo, 48-49.

276 Clark, 53.

277 Clark, 54.

${ }^{278}$ E. D. Edmund Dene Morel, The British Case in French Congo; the Story of a Great Injustice, Its Causes and Its Lessons. (New York: Negro Universities Press, 1969), 12-13.

${ }^{279}$ Morel, 12-13.

280 Great Britain. Naval Intelligence Division, French Equatorial Africa \& Cameroons, 228.

281 Great Britain. Naval Intelligence Division, 228.

282 Morel, The British Case in French Congo; the Story of a Great Injustice, Its Causes and Its Lessons., 14.
} 
ground-nuts in the region as well ${ }^{283}$. Slaves were the major export by 1517 but of a different sort than previously known and owned by people within the kingdoms ${ }^{284}$. The slave trade was abolished in England in 1807, in France in 1819, and in Portugal in 1836 but by 1840 approximately 150,000 slaves were still exported annually. By the middle of the nineteenth century the export of slaves from the west coast of Africa subsided ${ }^{285}$. In the seventeenth century France and England awarded large companies exclusive trade monopolies in the region but by 1769 the entire coast in the region was available to all ${ }^{286}$. French exploration of the coast and inland waterways continued under various private and official agreements for another century 287 .

\section{Colonial}

The colonial period is characterized by two timeframes: a concessionary region between 1880 and 1930, and an administrative and resistance headquarters from 1930 to 1960288 . In 1880, Pierre Savorgnan de Brazza of France negotiated a treaty with the king of the Makongo (one of the three kingdoms) for "protectorate rights in the local territory"289. Thus began the French concessionary companies, and their domination of trade in the "French Congo" until 1930. This area of French control

\footnotetext{
283 Great Britain. Naval Intelligence Division, French Equatorial Africa \& Cameroons, 229.

284 Great Britain. Naval Intelligence Division, 229.

285 Great Britain. Naval Intelligence Division, 229.

286 Great Britain. Naval Intelligence Division, 228.

287 Great Britain. Naval Intelligence Division, 231.

288 Clark, The Failure of Democracy in the Republic of Congo, 54.

289 Clark, 54.
} 
was subsequently carved out into four distinct colonies, administratively know as French Equatorial Africa: Moyen-Congo (later Republic of Congo), Oubangui-Chari (later the Central African Republic), Gabon, and Chad ${ }^{290}$. The concessions extracted wood, rubber, ivory and other resources at the expense of laborers who suffered in the French quest for more and more export material. The Congo Free State (later to become the Democratic Republic of Congo), a separate area south of the Congo River, was recognized in 1884. Prince Bismark presided over the Berlin Act of 1885 to minimize tensions between the French and Belgians as well as to outline conditions for trade and commerce in the Congo Free State 291 . The French and Belgians committed many atrocities alike in the pursuit of resource extraction and infrastructure projects like railways ${ }^{292}$. Revolts against colonial rule were frequent but subdued until the end of the concessionary system in 1930. The Congo then "became a 'normal' colony" and the Congolese had rights to communicate with authorities ${ }^{293}$. The second phase of the colonial period began in earnest around 1940 with the Nazi occupation of France. Brazzaville was the administrative hub of French Equatorial Africa at the time and it became the resistance headquarters of Free France.

\footnotetext{
290 Clark, 55.

${ }^{291}$ Morel, The British Case in French Congo; the Story of a Great Injustice, Its Causes and Its Lessons., 35.

292 Clark, The Failure of Democracy in the Republic of Congo, 57.

${ }^{293}$ Clark, 57.
} 


\section{Cold War}

After World War II, France slowly increased rights to its colonies and by 1960 Congo was independent ${ }^{294}$. During this second wave of colonial domain, Congolese resistance became more activist and political. An important figure during the resistance was Andre' Matsoua as he argued for increased rights until his death in a labor camp. He rekindled the "big man" persona in Congo ${ }^{295}$. Abbe' Fulbert Youlou, became the Congo's first president in 1956 and as such became a successor of sorts to Matsoua's legacy of spirited resistance ${ }^{296}$. Regional and "personalistic" politics, as well as regional alliances marked the late colonial period ${ }^{297}$. In 1958 the Congolese voted to become an autonomous state in the French Community, and shortly thereafter, Youlou became the prime minister. The new prime minister did not go undisputed however, and fighting occurred between different factions as Youlou decided to move the government seat to Brazzaville from Point Noire. In 1959, Youlou was elected president of the new republic and in the following years he established practices like arresting his rivals, then rehabilitating them by offering them government jobs, then removing them due to corruption that would persist throughout the Cold War era ${ }^{298}$. Congo continues in the tradition of Youlou's example with an emphasis on the patron-client relationship, ethno regional politics, the militarization of politics, and

\footnotetext{
294 Clark, 55.

295 Clark, 58-60.

296 Clark, 60.

297 Clark, 61-63.

298 Clark, 64-66.
} 
an enduring "psychological" dependence on its former colonizer ${ }^{299}$. Youlou was forced to resign after three days of massive protests against the failing economy. From 1963-1990, additional political and cultural attributes surfaced: monopartyism, and a young entitled class committed to securing a different future $^{300}$. In the post-Youlou government, Alphonse Massamba- Dèbat was the young new prime minister, soon to be president (ruling for five years) and Lissouba would become his prime minister. Massamba's popularity eroded over his time in office due to his unwillingness to satisfy the more radical groups, i.e., the Marxists and conservatives as he forged a socialist strategy and he resigned in 1968. He was replaced by August Poignet, then in short order by the twenty-nine year old Captain Marien Ngouabi. Ngouabi established a party based loosely on the Soviet Union's communist party and changed the country's name to the People's Republic of the Congo. The Marxist influence over time was not significant even though the state was committed to controlling the economy; ultimately it was "a perpetuation of existing patterns of authoritarianism in a new guise"301. Following in Ngouabi's steps after his assassination were Joachim Yhombi-Opango (1977-1979) and Denis Sassou-Nguesso (1979-1991) who also ran a halfhearted Soviet fashioned government but it was all a ruse to obscure the military dictatorship. SassouNguesso "was (and remains) a warlord politician" 302. He ruled until 1991 when

\footnotetext{
299 Clark, 68.

300 Clark, 68-70.

301 Clark, 70-72.

302 Clark, 72.
} 
there was conference that forced him to acknowledge a changing political landscape (mounting economic challenges in Congo, the end of the Cold War, the collapse of communism in the Eastern Bloc countries and a workers strike) that allowed democratic aspects ${ }^{303}$. The conference (presided over by Monsignor Ernest Kombo) allowed for the expression of democratic ideals during which time the single-party era, its institutions, and old political norms were weakened such that the constitutional reform and new elections could be organized. The conference was a start but not necessarily a break from the past. ${ }^{304}$.

\section{Post-Cold War}

Sassou's reign ended in 1992 when a temporary government was established to manage the transition to a new constitution at which time Pascal Lissouba was elected president. This transition was not without criticism, cheating was alleged, coups were thwarted, the army was curbed and that was just the beginning of the democratic experiment ${ }^{305}$. Former President Sassou and another competitor Bernard Kolèlas challenged the delicate and fledgling democracy. Kolèlas came in second in the second round of elections in 1992 and as such should have been name the prime minister but Lissouba decided to dissolve the Assembly instead and hold another election in the following year, thwarting Kolèlas. The presidential guard

\footnotetext{
303 Clark, 73.

304 Clark, 74.

305 Bruce A. Magnusson and John F. Clark, "Understanding Democratic Survival and Democratic Failure in Africa: Insights from Divergent Democratic Experiments in Benin and Congo (Brazzaville)," Comparative Studies in Society and History 47, no. 3 (2005): 561.
} 
fired upon demonstrators opposed to Lissouba's actions, three were killed ${ }^{306}$. In 1993, during another legislative election, Lissouba's coalition won but his opponents cried foul and shortly thereafter a militia group (the "Ninjas") loyal to Kolèlas purged some neighborhoods of Lissouba supporters, just as Lissouba's militia purged areas with Kolèlas supporters. Mediation by international political figures intervened and came to conclusion in the Libreville Accords on August 4, 1993.307 Additional elections ensued and Lissouba's party gained the majority of seats in the Assembly. Renewed violence in opposition spread between November 1993 and January 1994 and ethnic cleansing occurred "aimed at the representatives of rival ethno-regional constituencies"308. All the while, former President Sassou and his militia "Cobra" waited out most of the violence. By the end of January 1994 a relative peace was negotiated between Lissouba and Kolèlas, and again in January 1995 whereby Kolèlas and his party garnered some cabinet positions. From this time until early 1997 there was less violence until the next election when the “democratic experiment" was shattered ${ }^{309}$.

During the re-election campaign in 1997 violence broke out in the northern area of Cuvette between Sassou and Joachim Yhombi-Opango supporters where several people were killed before mediators were able to negotiate peace. The peace accord was broken less than a month later when Lissouba's forces went to Sassou's

\footnotetext{
306 Magnusson and Clark, 562.

307 Magnusson and Clark, 562-63.

308 Magnusson and Clark, 563.

309 Magnusson and Clark, 563.
} 
residence. Sassou and his Cobra fighters anticipated this move and were armed for the encounter; four months of civil war ensued. Kolèlas was neutral for a while but eventually he sided with Lissouba. Angola interrupted the standoff by sending thousands of troops in support of Sassou who was then able to take over essential areas by October, ensuring his success and control of the government ${ }^{310}$. The multiparty democratic experiment ended in 1997 with about ten thousand civilian casualties, hundreds of thousands people displaced from their homes, tens of thousands of homes destroyed, and about thirty thousand people seeking refuge in the Democratic Republic of Congo ${ }^{311}$. Sassou decided to consolidate his power rather than engage in legitimizing multiparty elections suggesting he might not have confidence he could win an election. He chose to assert himself and dominate over the population through fear but not first without another (and more deadly) civil war ${ }^{312}$. There have since been other civil wars: between December 1998 and November 1999, and again for about a year in 2002 over contested elections ${ }^{313}$. Presidential elections were also held in $2007^{314}, 2009,2016$ - Sassou won each election.

\footnotetext{
310 Magnusson and Clark, 564.

311 Clark, The Failure of Democracy in the Republic of Congo, 249.

312 Clark, 250.

313 Clark, 5.

314 Marie-Soleil Frère, "Covering Post-Conflict Elections: Challenges for the Media in Central Africa," Africa Spectrum 46, no. 1 (2011): 3.
} 
b. Description of macro-structural weakness

\section{Democracy}

One of the macro-structural weaknesses of ROC is that democracy has not been consolidated even though it had been a hope. The Republic of Congo is not considered a democracy because there has never been turnover of duly elected parties to another party ${ }^{315}$ even though there is a semi-presidential institutional form of government ${ }^{316}$. Congo sustained a decade of armed conflict from 1993 to $2003^{317}$ between militia fighting and outright civil war in 1997 in a battle to maintain the "multiparty experiment" started in the early 90 s $^{318}$. In 2002, a new constitution was created which fixed a seven-year presidential term limit and a bicameral national parliament. Irregularities in the 2002 and 2007 elections caused many to believe they were not legitimate ${ }^{319}$. In 2009 Sassaou was elected for a seven-year term. By 2015 another constitutional amendment by referendum established a five-year presidential term limit (down from seven years), and the ability to serve three terms instead of two was fixed. In 2016 Sassou was elected for his third term- this time for five years. The fact that the third term was created through "constitutional channels" demonstrates an awareness of global shifts in

\footnotetext{
315 Clark, The Failure of Democracy in the Republic of Congo, 6.

316 Magnusson and Clark, "Understanding Democratic Survival and Democratic Failure in Africa," 569. 317 UNICEF, "UNICEF Humanitarian Action Report 2008 Republic of the Congo Brazzaville," accessed April 11, 2019, https://www.google.com/search?client=safari\&rls=en\&q=unicef+humanitarian+action+report+200 $8+$ republic+of + the+congo+brazzaville\&ie=UTF-8\&oe=UTF-8. 318 Clark, The Failure of Democracy in the Republic of Congo, 2.

${ }^{319}$ Freedom House, "Freedom in the World: Congo, Brazzaville 2009," Freedom House, n.d., https://freedomhouse.org/report/freedom-world/2009/congo-republic-brazzaville.
} 
acceptance of power consolidation ${ }^{320}$. He is currently the president of Congo ${ }^{321}$.

Elections are "democratic" as there are multiparty candidates but the candidates do not have a real chance of winning. It might be considered "choice without choice"322 in this authoritarian regime. USAID gives ROC a liberal democracy score of .11 on a 0 to 1 scale $^{323}$. As of 2019, Freedom House concludes Congo is politically "not free" as its aggregate freedom score is 21 out of $100(0=$ least free $)$ with political rights 7 out of 7 , and civil liberties 5 out of 7 ( $7=$ least free $)^{324}$.

\section{Governance}

The macro-structural constraint of poor governance in ROC undermines many natural opportunities for the country to succeed. With a combined governance score of -1.19 on a scale of -2.5 to 2.5 from 1996 to 2014 , (from the average of the voice and accountability score and the government effectiveness score from the worldwide governance indicators), ROC ranks near the bottom of countries worldwide. A comparative discussion (Magnusson and Clark, 2005) of Benin and Congo's divergent paths after inaugurating fledgling democracies in the early 1990s

\footnotetext{
320 Daniel N. Posner and Daniel J. Young, "Term Limits: Leadership, Political Competition and the Transfer of Power," Institutions and Democracy in Africa: How the Rules of the Game Shape Political Developments, 2018, 274.

321 "Embassy of the Republic of Congo in Washington DC > About Congo > Government," accessed October 14, 2019, http://www.ambacongo-us.org/en-us/aboutcongo/government.aspx.

322 Irina Busygina, Mikhail Filippov, and Elmira Taukebaeva, "To Decentralize or to Continue on the Centralization Track: The Cases of Authoritarian Regimes in Russia and Kazakhstan," Journal of Eurasian Studies 9, no. 1 (2018): 64, https://doi.org/10.1016/j.euras.2017.12.008.

323 USAID, “Congo (Brazzaville), Journey to Self-Reliance: FY 2020 Country Roadmap," n.d., https://selfreliance.usaid.gov/country/congo-brazzaville.

324 Freedom House, "Congo, Republic of (Brazzaville)," Freedom in the World 2019, January 28, 2019, https://freedomhouse.org/report/freedom-world/2019/congo-republic-brazzaville.
} 
reveals that self serving leadership undermined institutions and exacerbated negative economic conditions and reckless IMR policies ${ }^{325}$. Corruption enriched the governing class particularly given the availability of natural resources. Although only a "modest producer" of oil it might be fair to say that the transition from autocracy to democracy was hindered by the corrupting power of easily accessible oil production ${ }^{326}$. Allegations of graft by the President's family from state oil contributed to the $159^{\text {th }}$ rank out of 180 countries in Transparency International's 2008 Corruption Perception Index ${ }^{327}$. Congo is one of the major oil producers in sub-Saharan Africa but unfortunately that does not translate to improved governance, rather it seems to detract from good governance. In fact a comparison study of data in Congo from 2004 and 2008 in Congo indicates that corruption worsened over the intervening years in this petro-state ${ }^{328}$. Oil revenue is observed to be a "personal cash cow" for the president and select friends, diverting over a billion dollars from Congo's budget ${ }^{329}$. USAID gives ROC a government effectiveness score of .27 on a scale of 0 to $1^{330}$. Unfortunately, even though ROC is an oil rich country the wealth of the nation does not normally translate to services for its citizens. It appears precisely because ROC

\footnotetext{
325 Magnusson and Clark, "Understanding Democratic Survival and Democratic Failure in Africa," 568.

326 Magnusson and Clark, 573.

327 Freedom House, "Freedom in the World: Congo, Brazzaville 2009."

328 Hazel M. McFerson, "Governance and Hyper-Corruption in Resource-Rich African Countries," Third World Quarterly 30, no. 8 (2009): 1530.

329 McFerson, 1534.

330 USAID, “Congo (Brazzaville), Journey to Self-Reliance: FY 2020 Country Roadmap."
} 
has natural oil resources it experiences short falls in social expenditure and public investment, due to corruption. Capital flight often caused by corruption is one enduring reason for expenditure shortages in Congo: first through colonial extraction of natural resources via forced labor, and then again more recently through oil revenue theft. Oil makes up 58 percent of the GDP and 78 percent of its exports. As a result the economy is very susceptible to oil shocks ${ }^{331}$ as well capital diversion. Capital flight reduces funds available for public spending and it undermines the country's tax base because there is less investment available for development. The availability of natural resources notoriously impairs judgment- it is sometimes called the "resource curse". It can motivate corrupt behavior and influence investment options that do not serve the public good, as well as further entrenching and slowing debt repayment programs due to loss of resources. Money spent servicing debt means that less money is available for public spending which results in higher IMR ${ }^{332}$. Moulemvo (2014) found capital flight in ROC occurs through foreign trade "misinvoicing", tax fraud, corruption, and debt. "Misinvoicing", (typically of oil exports) represented approximately US\$3,848 million in 2012 alone, and is facilitated by the difficultly in forecasting production and revenue by the Ministry of Finance and the Budget, and the Oil Ministry. Sophisticated tax fraud occurs through multinational companies, producers in the oil sector, and the Congo

\footnotetext{
331 "Republic of Congo : Selected Issues," IMF, 14, accessed October 29, 2019, https://www.imf.org/en/Publications/CR/Issues/2016/12/31/Republic-of-Congo-Selected-Issues41889.

332 André Moulemvo, "Impact of Capital Flight on Public Social Expenditure in Congo-Brazzaville," African Development Review 28, no. S1 (2016): 114.
} 
National Oil Company. Capital also leaves Congo through unlawful gains and transfers. Congo has also received debt assistance from international lenders but that money was "siphoned out of the country by the ruling elite" particularly in the 1980s which also exacerbated capital flight ${ }^{333}$.

With low transparency and accountability, development is not necessarily made a priority. Poor governance has ultimately led to low social spending, particularly compared to other countries in the region. For instance, in 2012, Congo spent 2.3 percent of GDP on public health spending, compared to Senegal at 5 percent, and Lesotho at 9.1 percent ${ }^{334}$. The condition of governance undermines investment in education and health as the nation's resources are siphoned off for illegal gain ${ }^{335}$.

\section{Economic Growth}

Finally, the economy is another component of macro-structural weakness in ROC. Underperforming at 4\% economic growth from 1996 to 2014 compared to $4.77 \%$ economic growth for 106 other countries during the same time, ROC has not kept pace. In the mid 1990s, the economy contracted annually and the civil war in 1997 also disrupted growth ${ }^{336}$. Growth performance was hindered by low years in oil production, weak fiscal planning, labor shortages due to expulsion of over 200,000 undocumented workers (which lead to a reduction in domestic demand), inability of

\footnotetext{
333 Moulemvo, 116.

334 Moulemvo, 116.

335 Moulemvo, 122.

336 Magnusson and Clark, 576.
} 
the treasury to pay invoices in timely manner, cash flow problems due to reduced revenues, and deteriorating quality of government financial data that prevents informed decision making ${ }^{337}$. Stifling economic growth since the 1980's was the "hangover of massive state investments, the creation of dozens of state enterprises, and a civil service payroll in excess of state liquidity" in addition to Congo's completely corrupt and bankrupt financial structure ${ }^{338}$. Oil wealth from natural resources provided employment and built up the middle class in the 1970s and 1980s; however it was the state, rather than the private sector, which was responsible for paying the wages of the middle class solidifying allegiance to the status quo 339 . An underachieving economy, curbed by poor governance, contributes to an underwhelming business environment score of .27 on a scale of 0 to 1 from USAID $^{340}$.

\section{c. Description of IMR decline}

Moving now from the complex history, and substantial macro-structural weaknesses of ROC, this research considers the reduction in IMR against the backdrop of national constraints. We turn our focus to the remarkable IMR improvement in ROC and how it potentially came to be: from 71.2 in 1996 and 34.4

\footnotetext{
337 Fulbert Tchana Tchana and Etaki Wa Dzon, "Republic of the Congo - Report Monitoring the Economic and Financial Situation" (The World Bank, December 4, 2015), 15-18, http://documents.worldbank.org/curated/en/211851467992000410/Republic-of-the-CongoReport-monitoring-the-economic-and-financial-situation.

338 Magnusson and Clark, "Understanding Democratic Survival and Democratic Failure in Africa," 559.

339 Magnusson and Clark, 574.

340 USAID, “Congo (Brazzaville), Journey to Self-Reliance: FY 2020 Country Roadmap.”
} 
in 2014341. For comparison, neighboring Central African Republic had an IMR of 115.4 in 1996 and 93.5 in 2014.

Given the IMR improvement in the context of ROC dysfunction, perhaps IMR is not the measure or proxy of development that we thought it was. Maybe IMR is a better measure of enclave development, say in health care, than for overall development indicator of a country. Enclave development is a process of a specific sector or region of a country improving, perhaps beyond other sectors or geographic areas, due to specific interventions (frequently from external partners who control quality and processes). Endogenisation, on the other hand, is an incremental institutional process in country whereby established standards are met through continued improvement, often through engagement with external partners ${ }^{342}$. Enclave development encouraged by international elite partners is the flip side of development endogenisation encouraged through improved vertical and horizontal governance channels from within a country. Both enclave and endogenious development are useful and they need not occur at the same time. Enclave development has detractors because of its perceived exclusivity and elements of control. Enclave development can both help and hinder development in countries. A few examples illustrate the positive and negative aspects of enclave development. In

341 UNICEF, “Unicef Humanitarian Action Report 2008 Republic of the Congo Brazzaville,” accessed April 11, 2019,

https://www.google.com/search?client=safari\&rls=en\&q=unicef+humanitarian+action+report+200 8+republic+of+the+congo+brazzaville\&ie=UTF-8\&oe=UTF-8.

342 Ayelech Tiruwha Melese and A. H. J. (Bert) Helmsing, "Endogenisation or Enclave Formation? The Development of the Ethiopian Cut Flower Industry*," The Journal of Modern African Studies 48, no. 1 (March 2010): 61, https://doi.org/10.1017/S0022278X09990218. 
Ethiopia for example, sector development came from an enclave of a cut flower industry, encouraged through Dutch foreign investors and development partnership, that flourished due to a value chain of "production, trading, and institutional sector development" 343 which allows the Netherlands to essentially control the entire silo of the cut flower industry. The development of an enclave has likewise had an effect, albeit slow, on the endogenisation of improved governance at the local level in Ethiopia through linkages with elite partnerships that help to institutionalize international norms ${ }^{344}$. The positive is that the Dutch provided structure and created a market for Ethiopian cut flowers, and national institutional processes improved. A disadvantage is that the Dutch control that specific sector silo in Ethiopia. Another example, this time from Zimbabwe demonstrates improvement in IMR came from endogenous health sector focus as seen over a period from 1982 to 1988. After independence, the Ministry of Health in Zimbabwe made the strategic decision to focus on primary care to deliver improved public health services, particularly to those previously underserved. Funding was decentralized to local sources to ensure more robust community based care. As a result, IMR dropped from 90 per 1000 in 1980 to 53 per 1000 in $1988^{345}$. The public health sector was a particular focus of the government for a time and with it came

\footnotetext{
343 Melese and Helmsing, 61.

344 Melese and Helmsing, 63.

345 Godfrey Kanyenze et al., Beyond the Enclave towards a Pro-Poor and Inclusive Development Strategy for Zimbabwe (Harare: Weaver Press in association with Alternatives to Neo-liberalism in Southern Africa, Labour \& Economic Development Research Institute, Zimbabwe and the Zimbabwe Congress of Trade Unions, 2011), 367.
} 
exceptional health outcomes in IMR. Public health spending increased from 2.2 percent to 3.0 percent over the 1980s, there was an increased emphasis on preventative and rural care, more infrastructure and more trained health workers ${ }^{346}$. An unintended consequence of the health sector focus was that there was not enough "institutional and political capacity" or money on the horizon to move the agenda forward to ensure all populations continued to receive comprehensive care ${ }^{347}$. That is when, in the early 1990 s, gains in health care started to tumble, as well as health indicators: in 1991 the early neonatal mortality rate shot up from 14.4 percent to 19.6 percent in $1996^{348}$. Interestingly, the endogenisation of the health care sector early on in the independence of Zimbabwe did not exclude allowing mission hospitals (the largest provider of private not-for-profit health care facilities in country). It was these same mission hospitals, primarily in rural areas, that covered for public health when it declined significantly by 1996. Funding from private donors was redirected from the failing public health system to the mission health care facilities that continued to thrive even though the public health system was in decline ${ }^{349}$. The enclave development of the health care sector provided a lifeline to many, a definite success but also it served as a diversion of funds that had previously supported an ambitious public health program that crumbled under its

\footnotetext{
346 Kanyenze et al., 368.

347 Godfrey Kanyenze et al., Beyond the Enclave towards a Pro-Poor and Inclusive Development Strategy for Zimbabwe (Harare: Weaver Press in association with Alternatives to Neo-liberalism in Southern Africa, Labour \& Economic Development Research Institute, Zimbabwe and the Zimbabwe Congress of Trade Unions, 2011), 368.

348 Kanyenze et al., 370.

349 Kanyenze et al., 370.
} 
own weight. Much like the examples in Ethiopia and Zimbabwe, with funding, international partnerships, and decentralization, it is not unlikely that the health sector and thus health outcomes improved, even in crisis conditions in ROC. In less than two decades the Republic of Congo more than halved the IMR under crisis conditions. This case of the Republic of Congo explores longitudinal data primarily from the Demographic Health Surveys (DHS) from $2005^{350}$ and 20112012351, with some data points from the Multiple Indicator Cluster Survey (MICS) from 2014-2015 ${ }^{352}$. See Appendix B The longitudinal data collected from the Demographic Health Surveys is organized here into four stages representing the chronology of care for women of childbearing age: preconception, antenatal care, delivery and postnatal care. The Demographic Health Surveys do not organize their data as such but it is useful categorize the information into four categories to try to identify where significant improvements were made. Using DHS and MICS data, research articles and reports help build a story of a country in transition to improved health outcomes for infants. This case study explores how the IMR in the Republic of Congo improved at the micro level and what conditions favored or buffered the chaos from the macro level. Essentially,

\footnotetext{
${ }^{350}$ Centre National de la Statistique et des Études Économiques- CNSEE/Congo and O. R. C. Macro, “Congo Enquête Démographique et de Santé 2005," July 1, 2006, https://dhsprogram.com/publications/publication-FR182-DHS-Final-Reports.cfm.

351 Centre National de la Statistique et des Études Économiques- CNSEE/Congo and I. C. F. International, “Congo Enquête Démographique et de Santé 2011-2012," December 1, 2012, https://dhsprogram.com/publications/publication-FR267-DHS-Final-Reports.cfm.

352 Institut National de la Statistique et UNICEF, "Enquête Par Grappes à Indicateurs Multiples (MICS5 2014-2015), Rapport Final." (Brazzaville, Congo: Institut National de la Statistique et UNICEF, 2015).
} 
conditions existed at the community level that facilitated the implementation of indirect mechanisms that alleviated the proximate factors of infant mortality to save lives while keeping overarching national conditions at arms length. See Appendix C.

d. Description of indirect mechanisms that alleviate the proximate causes of infant mortality

Between the first Demographic Health Survey in Congo (DHS) in 2005 and the second DHS in 2011-2012 a number of indirect mechanisms (both health, and social and policy mechanisms) alleviated the proximate causes of infant mortality were improved as demonstrated through the chronology of care for women of childbearing age, i.e., preconception, antenatal care, delivery, and postnatal care. The comparable data reveal changes over time and provides clues as to how the IMR improved substantially even in crisis conditions. Many of the improvements in health care appear modest and they occur unevenly, while other changes appear more robust in the chronology of care to impact IMR.

\section{Preconception}

Against the backdrop of crisis in ROC, this research finds that there was little improvement in care in this preconception phase between the two surveys based on compiled responses. In the preconception phase, the change over time between the two surveys show that family planning messages heard through media were down significantly, even though broadly speaking readership and TV viewing was up. Birth intervals, an important aspect of healthy pregnancy remained relatively 
unchanged, as did the age of first birth. Contraception knowledge was slightly improved although it was already fairly universal.

Family planning messages shared through media like television and radio (especially in societies with emphasis on oral tradition) can be an important way to communicate health information however media used as a venue for sharing family planning information was under utilized. As literacy rates held steady in ROC at $79.3 \%$ between 2006 and $2016^{353}$, the low literacy rate suggests that family planning messaging via television and radio could be useful to reach target populations. Family planning messages heard through media (radio, TV, journals, and magazines) all decreased although women exposed to media who read

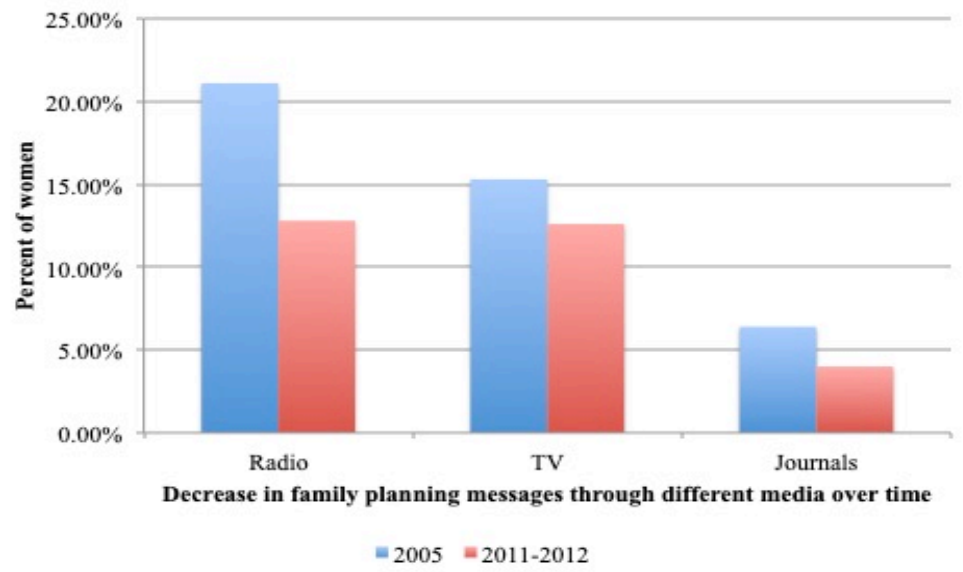

newspapers and watched TV increased.

Figure 7: Decrease in family planning messages through different media over time

353 UNDP, "Human Development Indices and Indicators: 2018 Statistical Update | Human Development Reports," 2018, http://hdr.undp.org/en/content/human-development-indicesindicators-2018-statistical-update. 
Another surprising find is that birth intervals were relatively unchanged over the span of the two DHS reports. The surveys cover only six years but other areas of the surveys demonstrate remarkable improvement (as seen in later phases of the chronology of care) during the same period. The percentage of women who had birth intervals between 7 months and 17 months (a very compromising interval as the medical community recommends at least a 24 months) was largely unchanged at $5 \%$.

Overall, most women in the Republic of Congo had knowledge of some type of contraception, and it improved slightly over time. In $2005,97.5 \%$ of women had knowledge of any type of contraception compared to 2011-2012 when slightly more women did at 98.6\%. Knowledge of modern and traditional contraceptive methods increased. However, the almost universal knowledge of contraception is contrasted by the actual contraceptive prevalence rate of $30.1 \%$ as of $2014^{354}$.

The surveys reveal that women in ROC did not wait longer for their first pregnancy. The age of first birth remained essentially unchanged between the two DHS reports: at around 19 years of age. The data suggests that education to shape attitudes and preferences about birth intervals and the age of first pregnancy were unaffected by efforts to change. Education about family planning might not be received well or trusted. Given that there is almost universal knowledge of contraception (which implies that there was education of some sort), the fact that the age of first birth

354 CIA, “The World Factbook," CIA, accessed November 20, 2019, https://www.cia.gov/library/publications/the-world-factbook/geos/cf.html. 
persists below the recommended age suggests that the message or manner of message did not resonate with the intended audience. Women may have preferred to wait longer for their first pregnancy but family or cultural considerations might take priority. Education to shape health outcomes and behavior might have a longer horizon than the survey span allows, not mentioning cultural attitudes towards increased maternal age for first pregnancy. Perhaps the improvement made in later care phases indicates that improving health outcomes are easier to facilitate through improving the quality of medical service delivery than through education for behavioral change in the preconception phase.

The preconception phase does not demonstrate aggressive change in ROC between data available in the two Demographic Health Surveys. Preventative action probably impacted birth intervals and contraceptive knowledge but not the age of first birth. When young women are pregnant they frequently access more health care through primary care, and community health workers at which time they learn about the importance of family planning and birth intervals but it seems to come after the fact. As media messages of family planning were down, preventative actions might have come from primary care providers, community health workers or perhaps through participation in women's health groups in the community but they were not particularly effective for these issues as measured in the DHS. 


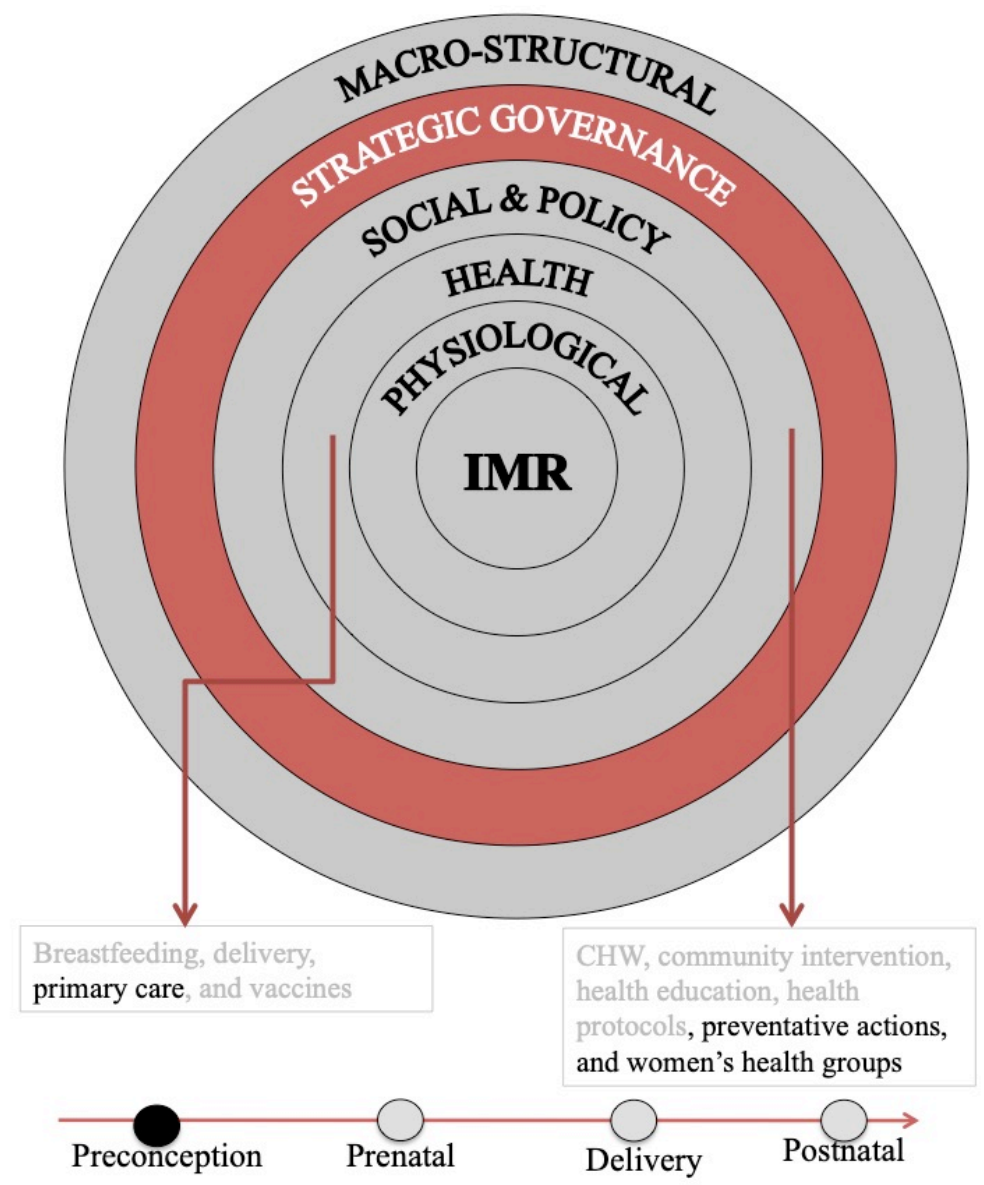

Chronology of potential care phases during a woman's life

Figure 8: Strategic governance at preconception phase (ROC)

\section{Antenatal Care/Prenatal Care}

The antenatal care/prenatal care phase, on the other hand, showed some significant improvements between 2005 and 2011-2012. Women received more quality care as represented by improved health protocols during their pregnancy through additional antenatal care visits and increased tetanus vaccinations. Moreover, health protocols improved as a consequence of more antenatal care visits spent with a trained medical professional. This increase in quality antenatal care (ANC) 
engagement could explain the substantial improvement in knowledge of pregnancy complications.

Importantly, the number of women who had no ANC visits decreased. For this measurement to decrease by more than a third suggests that the most disenfranchised women were able to engage more with the health community, probably through interactions with trusted community health workers in rural settings. If women were unable to participate in at least one ANC visit that suggests that their circumstances were particularly dire. However, more women were receiving care, and from increasingly more qualified medical professionals. Those who received ANC care from doctors and mid-wives increased, while care from a nurse decreased slightly. Women received more ANC care in the space of six years. The number of women who, during their last pregnancy, received two or more tetanus toxoid injections increased. While later data from the 2014-2015 Multiple Indicator Cluster Survey (MICS) shows that the number of women who, during their last pregnancy received two or more tetanus toxoid injections increased further which is an encouraging development for IMR. Receiving two tetanus injections protects a pregnant mother and baby from tetanus, which is a very real threat in childbirth in ROC. 


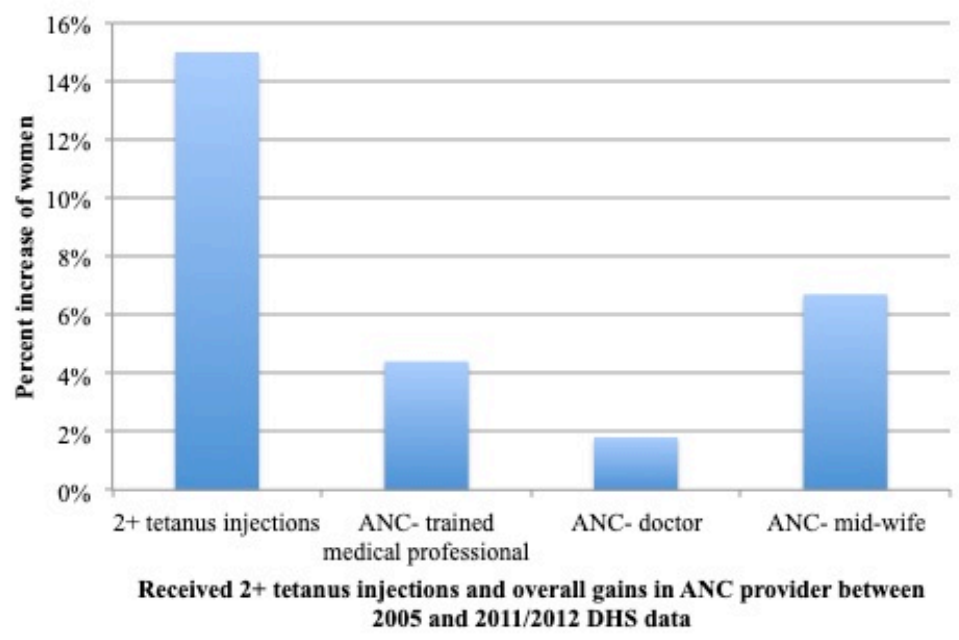

Figure 9: Received 2+ tetanus injections and overall gains in ANC provider between 2005 and 2011/2012 DHS data

There was more knowledge about complications of pregnancy. Understanding complications allows pregnant women to make informed choices about pursuing skilled care sooner rather than later. Unusually high blood pressure, or unexpected bleeding (for instance) could signal health emergencies in pregnant women. If women understand that these symptoms are problematic they can seek care, or start to arrange to seek care rather than waiting until symptoms are especially extreme. In rural areas, skilled medical care may be far away and require the coordination of different types of transportation from various family members or neighbors. The coordination effort takes time and in emergency situations time is of the essence. Knowing complications of pregnancy can help women anticipate their needs sooner and problem solve for resolution. In some countries women have started emergency bicycle ambulance groups to support pregnant women as a way 
to address shortfalls in emergency transportation. In 2005 , only $40.2 \%$ knew about different complications versus 62\% in 2011-2012.

Routine antenatal care at appointments became more standardized through attention to detail. Performance improved at the point of care which points to improved health protocols. Improvement of straightforward routines suggests specific attention was given to achieve across the board implementation. Health workers were able to boost measureable results by simply following recommended health visit protocol without requiring additional knowledge of obstetrics. This goes to the point that improving the IMR is more about implementation science rather than new medical technology. Some indirect mechanisms that seem to alleviate the proximate causes of infant mortality between the two surveys point to improved health protocols (from primary care to the community health worker), increased health education of medical personnel and pregnant women, preventative actions, and ultimately increased participation with the health system. 


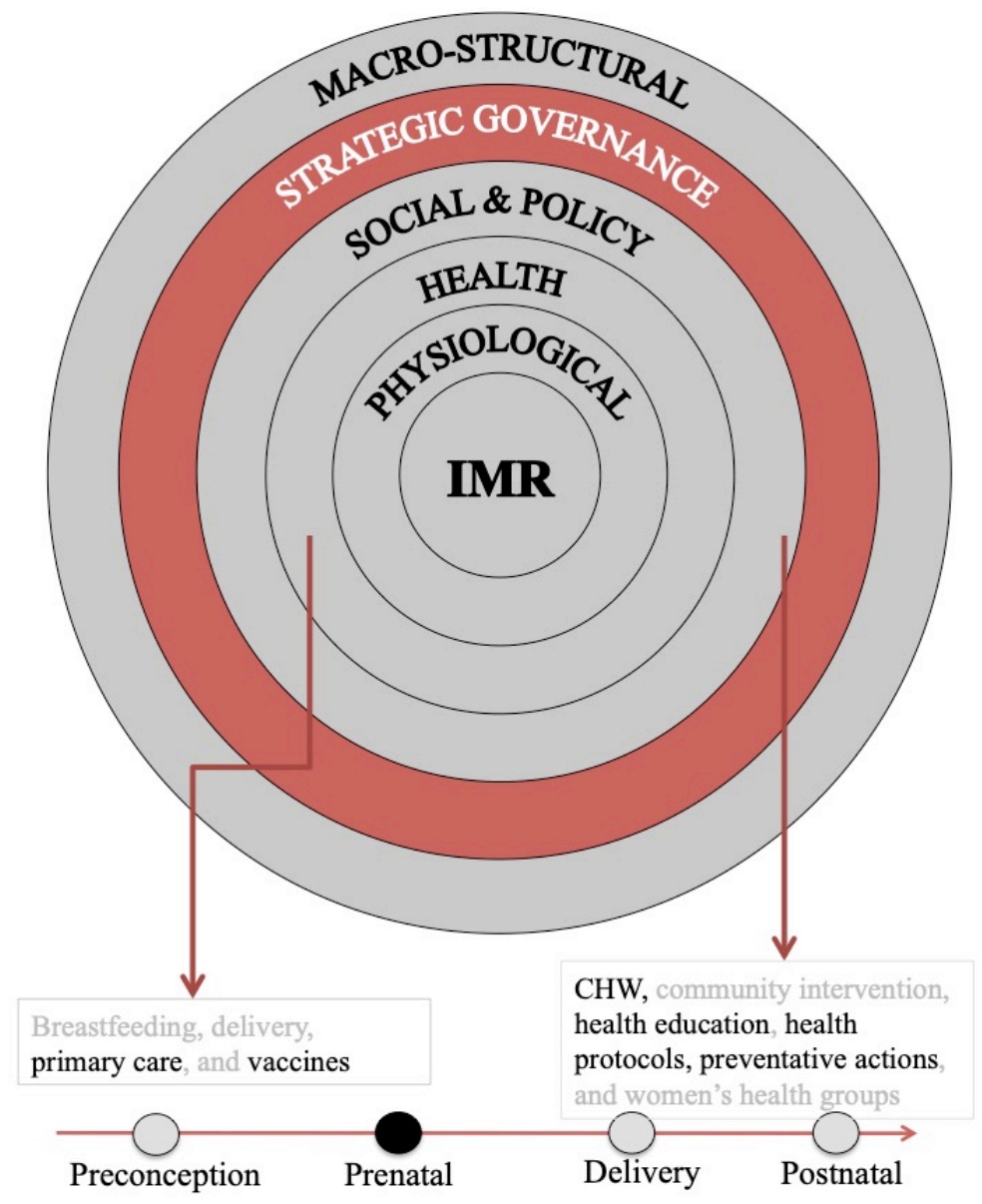

Chronology of potential care phases during a woman's life

Figure 10: Strategic governance at antenatal/prenatal phase (ROC)

\section{Intrapartum/Delivery}

In the critical and time sensitive delivery phase in the chronology of care, notable improvements were made as demonstrated by DHS data. Maternal mortality decreased by 55\%, health facility births increased while home births declined, and assisted deliveries increased. Everything improved in the right direction. Improved health protocols at delivery and primary care made a substantial difference in 
health outcomes. This phase in the chronology of care unequivocally changed health outcomes for the better.

Maternal mortality decreased from 781 per 100,000 to 426 per 100,000 . The sharp decrease in maternal mortality immediately impacts infant mortality. Newborns without mothers are very much at risk of death due to lack of care and feeding. Improved maternal mortality represents much improved health protocols at delivery.

Health facility deliveries increased in both the public and private sector. Women had more access to health facilities and were educated about the importance of facility births providing necessary interventions.

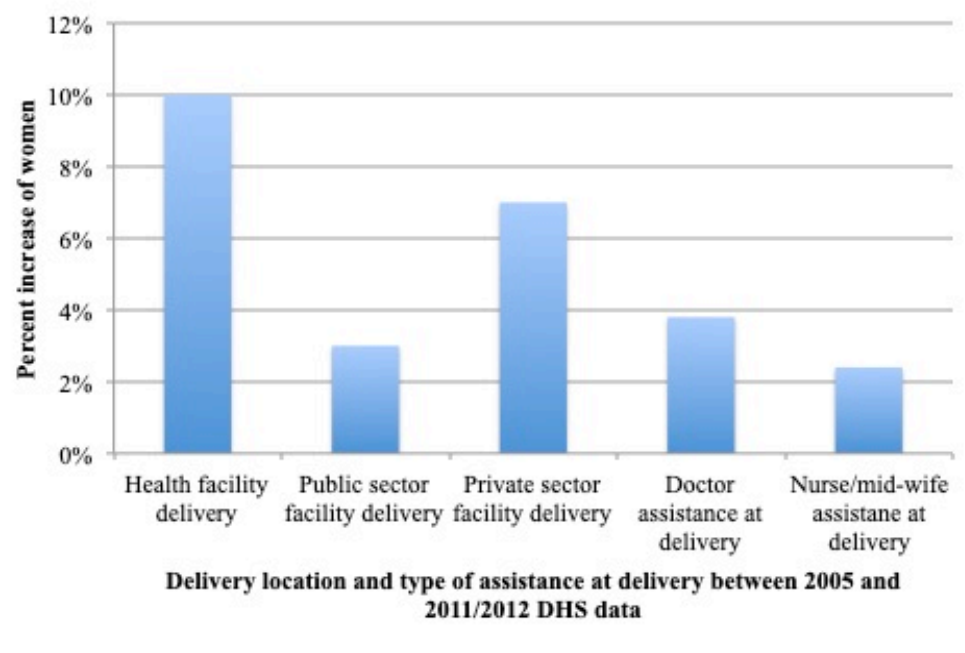

Figure 11: Delivery location and type of assistance at delivery between 2005 and 2011/2012 The trend towards universal facility birth leads one to believe that women in Congo had increasing faith and trust in their health providers, and as women experienced safer deliveries more women were inclined to have facility births. Indirect 
mechanisms appear to alleviate the proximate causes of infant mortality through improved health protocols, health education, primary care and delivery.

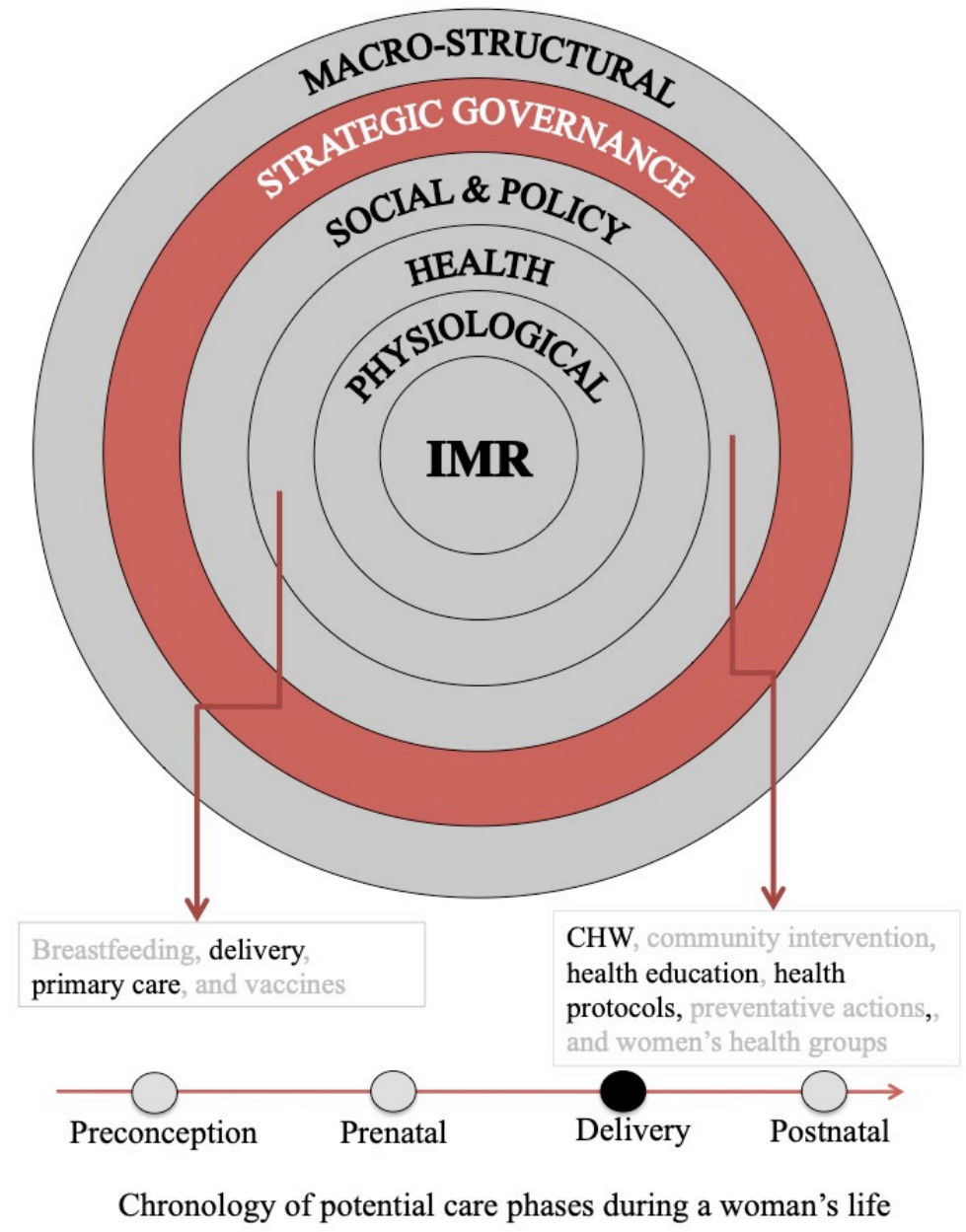

Figure 12: Strategic governance at delivery phase (ROC)

\section{Postnatal}

Finally, in the postnatal phase in the chronology of care, there was demonstrated improvement in care for infants even though infant vaccination was uneven. In this phase, as in all phases of care, an ounce of prevention is like a pound of cure in ROC. In ROC it can be a challenge for some infants to have access to quality medical 
treatment, let alone the indicated treatment to cure deadly health conditions. It is more prudent to prevent disease and illness in the first place than to expose an unprotected infant to compromised circumstances because recovery is an uphill battle under any circumstance. The two surveys demonstrate that vaccination, and the knowledge of oral rehydration salts, malaria prevention, and HIV transmission through breastfeeding improved considerably.

For instance, infant vaccination, for the most part, increased between 2005 and 2011-2012. Vaccines administered for tuberculosis, for diphtheria, tetanus, and pertussis, for measles, and yellow fever all increased. Contrary to the encouraging improvements mentioned above, infants receiving no vaccination increased and polio vaccination declined. The uneven vaccination rate hints at different trends of various immunizing partners, and the difficulty emphasizing routine health and follow up care of infants and children.

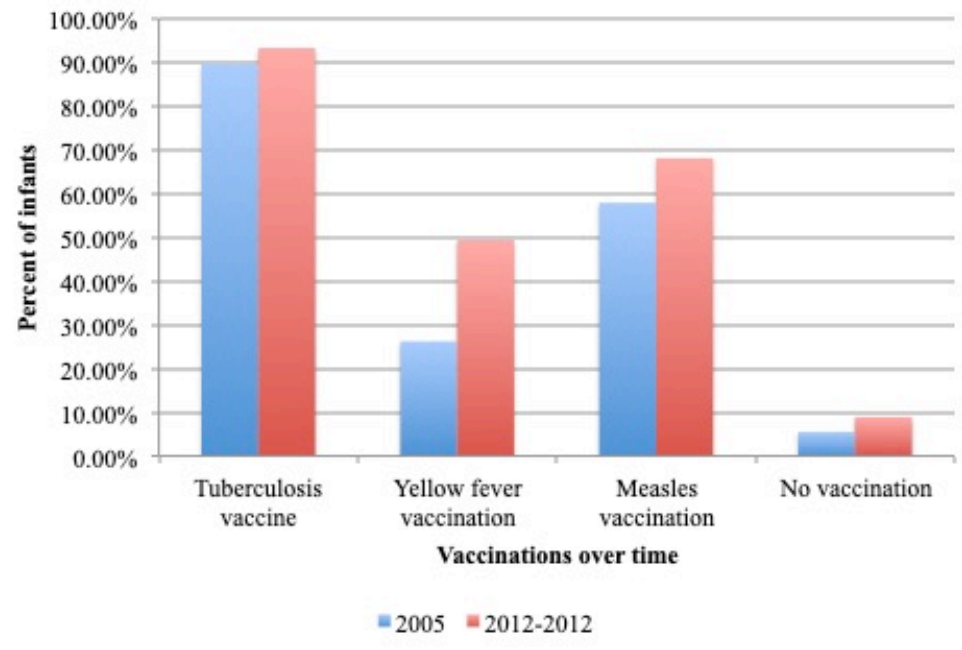

Figure 13: Vaccinations over time. 


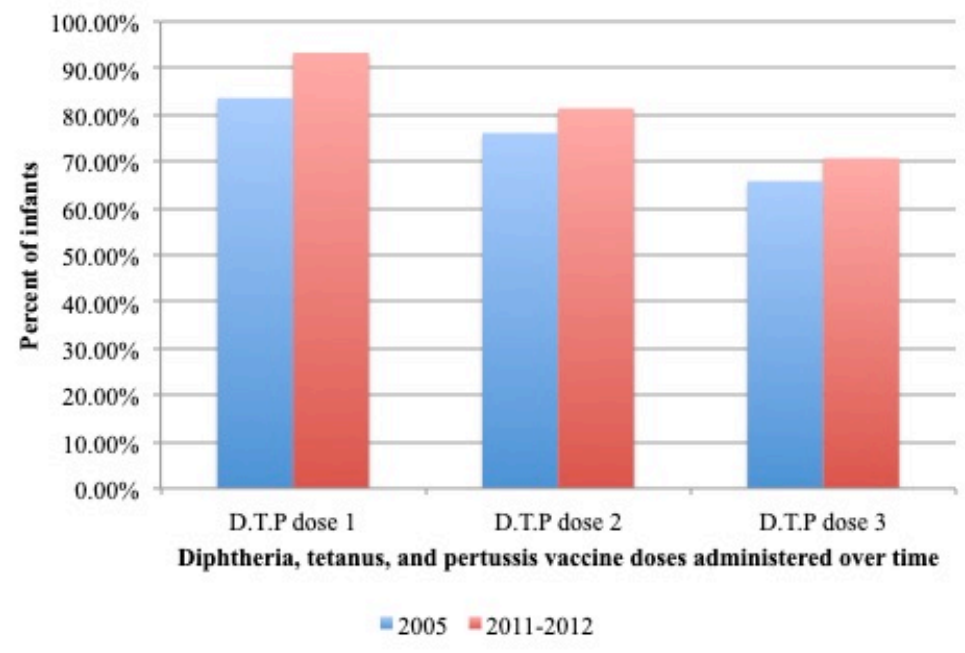

Figure 14: DTP doses over time

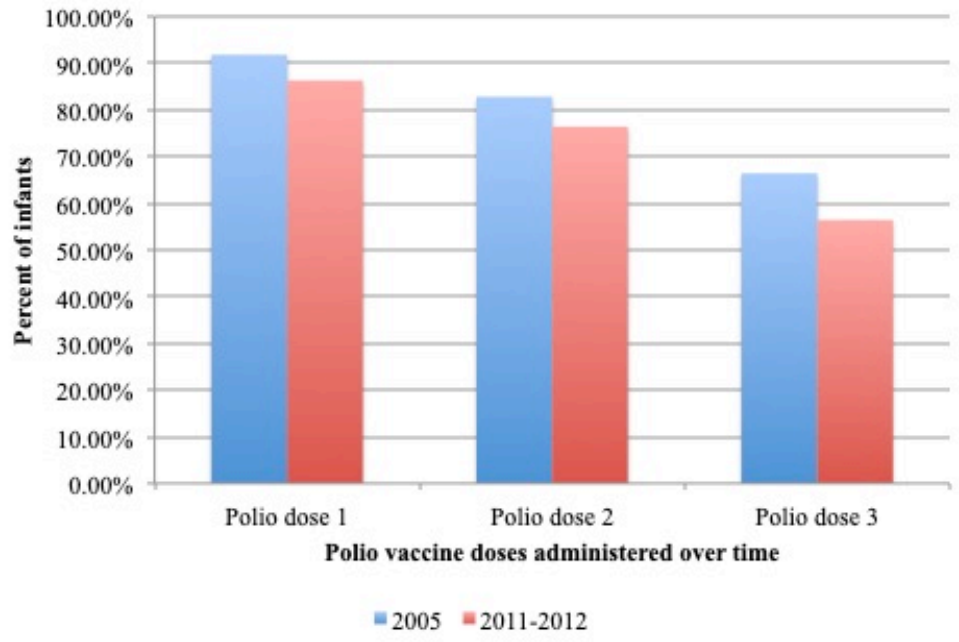

Figure 15: Polio doses over time

Disease (frequently incurred when vaccination is not available) and infection can cause diarrhea having a devastating impact on infants. Oral rehydration salts developed in the 1970 s were designed to treat acute dehydration from diarrhea ${ }^{355}$

355 Henry J. Binder et al., "Oral Rehydration Therapy in the Second Decade of the Twenty-First Century," Current Gastroenterology Reports 16, no. 3 (February 22, 2014): 375, https://doi.org/10.1007/s11894-014-0376-2. 
and this therapy has been hailed as one of the greatest therapeutic achievements of the twentieth century ${ }^{356}$. Therefore, increased knowledge of ORS is a path to improved health for infants with acute diarrhea. In ROC, knowledge of oral rehydration salts (ORS) improved substantially from $56.4 \%$ to $69.3 \%$. ORS use undoubtedly helped ease suffering and saved infant lives once parents knew about the dangers of dehydration from diarrhea - shockingly, the cause of death for $7.5 \%$ of children in ROC and the third leading cause of healthcare visits for children after malaria and respiratory infections ${ }^{357}$. Diarrhea in ROC is a symptom of contaminated water, unsanitary conditions, viruses, disease, and compromised health in general. The burden of acute gastroenteritis, and subsequent diarrhea, is particularly high for children under 5 in sub-Saharan Africa ${ }^{358}$. Therefore, the increase in knowledge of ORS is especially vital for preventing the harshest consequences of diarrhea in infants.

In addition to environmental contaminants the ubiquitous mosquito afflicts countless people in sub-Saharan Africa by transmitting deadly disease. For the lucky few, sleeping under nets can minimize exposure to mosquitos but cost and quality (e.g., holes in the net) can render them of marginal effectiveness. Prevention in this

\footnotetext{
356 Lancet, "Water with Sugar and Salt," The Lancet 312, no. 8.084 (1978).

357 Vivaldie Mikounou Louya et al., "Molecular Characterization of Norovirus Infection Responsible for Acute Diarrhea in Congolese Hospitalized Children under Five Years Old in Brazzaville, Republic of Congo," International Journal of Infectious Diseases 88 (November 1, 2019): 42, https://doi.org/10.1016/j.ijid.2019.07.034.

358 Mikounou Louya et al., 41.
} 
case is the best medicine. In ROC there was an increase in the number of people who had at least one mosquito net, from $75.5 \%$ to $83.9 \%$.

Education in the area of HIV transmission can improve IMR particularly in the areas hardest hit in sub-Saharan Africa. Approximately 3.7\% of women aged 15 to 49 in Congo have HIV, which contributes to the 66,000 AIDS orphans aged 0 to 17359 . Women between 15 and 49 years of age who know that HIV can be transmitted by breastfeeding increased as did their knowledge of special drugs to reduce vertical transmission of HIV in both urban and rural areas.

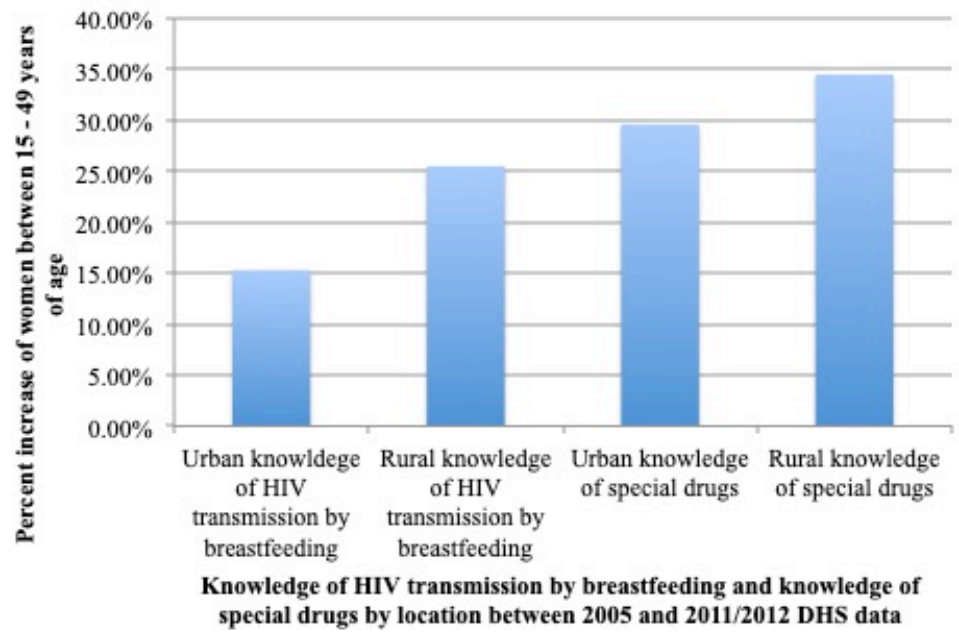

Figure 16: Knowledge of HIV transmission by breastfeeding and knowledge of special drugs by location between 2005 and 2011/2012

The indirect mechanisms contributing to the alleviation of the proximate physiological mechanisms of infant mortality in the postnatal phase include breastfeeding, primary care, health education and preventative actions in areas of

359 "Congo | UNAIDS," accessed November 25, 2019, https://www.unaids.org/en/regionscountries/countries/congo. 
oral rehydration salts and vertical HIV transmission, and malaria prevention, as well as improved health protocols and improved vaccination in many cases.

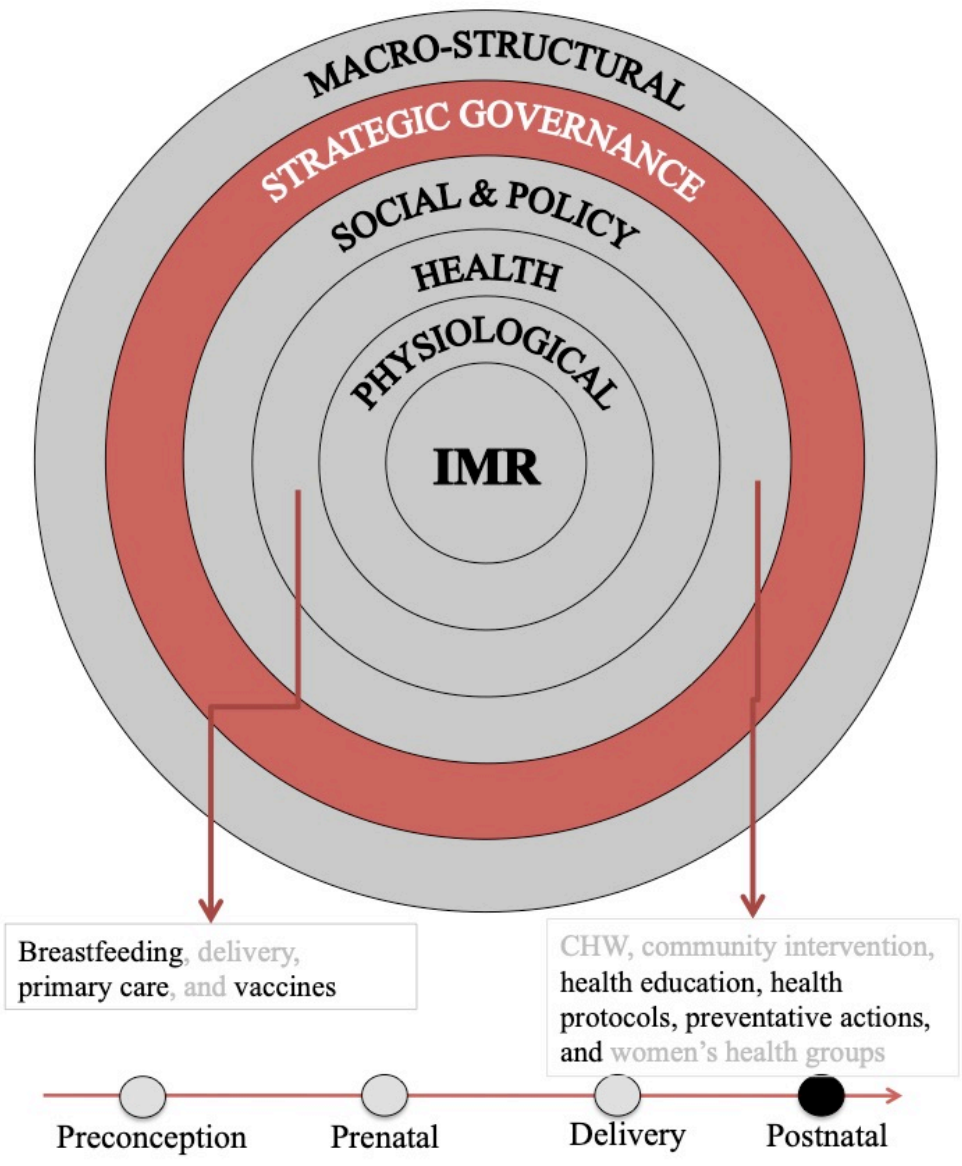

Chronology of potential care phases during a woman's life

Figure 17: Strategic governance at postnatal phase (ROC)

e. Case study process tracing and analytic narrative development

Now through the procedure of analytic narratives, we look for institutional drivers in ROC that have a plausible causal relationship to the outcomes of note, in this case the indirect mechanisms that alleviate the proximate mechanisms of infant mortality. In the process of uncovering the indirect mechanisms that alleviate the 
proximate mechanisms of infant mortality in ROC several clusters emerge that might explain institutional drivers as particularly useful when macro-structural conditions were bad. By observing micro governance patterns that relate directly to IMR we are able to identify these causal drivers. Through a review of health-related governance literature in Congo between 1996 and 2019, five key institutional drivers were identified. Each showed a repeated pattern of causal influence over the indirect mechanisms that alleviate infant mortality. They are: decentralization, international partnerships, funding, performance-based funding (PBF), and cultural change. These five institutional drivers have a mutually reinforcing effect; one enables the other although there is probably not a specific sequence.

\section{The Narrative: Part I Decentralization}

One probable driver of change that impacted the indirect mechanisms that alleviate the proximate indicators of infant mortality in ROC was decentralization. Devolving administrative responsibility for health care provision from the national government to the local level seems a likely antidote to dysfunction. Given that democracy was in crisis, governance was defunct, and economic growth was below average, the idea and/or practice of gradually distributing some authority away from the hub of said dysfunction might prove beneficial. Moving the power of decision-making away from central control was part of a democratic movement 
worldwide with the fall of communism ${ }^{360}$. This devolution included political, economic and administrative powers that were pushed down to local governments

\section{Analyzing the Narrative: Decentralization}

Decentralization influences the indirect social and policy mechanisms that alleviate infant mortality by impacting health education, intervention opportunities, preventative actions, and improved health protocols. Indirect health mechanisms that alleviate the proximate mechanisms of infant mortality in ROC are impacted by decentralization are vaccines and primary care. Decentralization is valuable and encourages sustaining partnerships at different levels of government. Dysfunction at the national level, which could dissuade partners from collaborating, can be circumvented to an extent and facilitates increased partnerships and funding because of perceived accountability. Through decentralization, the entire chronology of potential care phases during a woman's life is impacted. One might wonder why Sassou, an authoritarian, would permit decentralization in the first place after a hard won takeover in 1997? We will never know his specific motivation but what might be assumed is that he wants to maintain power and that decentralizing some power and authority to the localities must facilitate his interests in one way or another. Let's consider the "contradicting incentives" 361 that might motivate an authoritarian government to either decentralize or centralize:

\footnotetext{
360 Busygina, Filippov, and Taukebaeva, “To Decentralize or to Continue on the Centralization Track," 61.

361 Busygina, Filippov, and Taukebaeva, "To Decentralize or to Continue on the Centralization Track," 62.
} 
decentralization can erode authority and strengthen political rivals, but at the same time it can prevent dispersed coalitions consolidating power against central leadership ${ }^{362}$. Understanding why or how authoritarian regimes are incentivized to decentralize or not can issue broadly from three motives. First, the global demand for democratization and the need to modernize often necessitate decentralization as governments look for efficiencies and cost savings at local levels. Second, decentralization by authoritarian governments can be a political response to undermine or placate threats to central leadership. Third, external coercion via donors, international organizations, norms and markets also explain why authoritarians might choose or be forced to decentralize ${ }^{363}$. Sassou decentralized his government officially, over time through constitutional referendum, even as he consolidated his position of leadership.

One argument for his decision to permit decentralization might have been to realize the success other countries had improving international indicators of health like maternal and infant mortality ${ }^{364}$. Maybe more likely, it was not the specific health outcomes he was looking to achieve but rather the legitimacy of his overextended tenure as leader of ROC. More constitutional rights extended to localities expresses a level of magnanimity and modernity on par with more democratic countries that are frequent donors to the region.

\footnotetext{
362 Busygina, Filippov, and Taukebaeva, 63.

363 Busygina, Filippov, and Taukebaeva, 63.

364 Cordaid, "Healthcare Approach Successful in Congo Brazzaville," Cordaid, 2013, https://www.google.com/search?client=safari\&rls=en\&q=healthcare+approach+successful+in+cong o+brazzaville\&ie $=$ UTF-8\&oe $=U$ TF -8 .
} 
Decentralization, or at least the appearance of decentralization, was likely part of "playing the game" for an international audience that values norms of democracy ${ }^{365366}$. Decentralization brought two different outcomes. First, Sassou further consolidated his position through a dispersed coalition of supporters as part of the locally elected leadership/elite. This has a threefold purpose of aiding in the specter of democracy, it legitimizes and places responsibility on local leaders through elections, and at the same time it can minimize the culpability of the central government when things go awry. The other outcome of decentralization is that it can unintentionally reinforce international norms that push back against authoritarian regimes, and it makes local level government partnership with international alliances attractive and/or necessary. Local governments can comply with international agendas through partnerships that can erode confidence in the central government; therefore, authoritarian leaders carefully weigh how much they are willing to decentralize to the local level, doing so only when it is politically or economically expedient ${ }^{367}$.

Whatever the motivation, the progression of detailed responsibilities to the local level represents increased opportunity and obligation to attend to local concerns; but in order for that to work local governance needs to function better than national

\footnotetext{
365 Clark, The Failure of Democracy in the Republic of Congo, 250.

366 Larry Diamond, "The Rule of Law versus the Big Man 1," in In Search of Democracy (Routledge, 2015), 254.

367 Busygina, Filippov, and Taukebaeva, "To Decentralize or to Continue on the Centralization Track," 63.
} 
governance and in many cases it does ${ }^{368}$. Diamond (2015) notes that

decentralization can improve poor governance when control and assets are shifted or shared to lower levels of government ${ }^{369}$, which can improve efficiency, effectiveness, transparency and accountability. As demonstrated later in the case, performance-based funding of health care programs can thrive in environments of increased transparency and accountability at local levels.

Some examples illustrate the impact of decentralization of IMR, as well as the chronology of care for women. A study (2007) on infant immunization demonstrates that regional governments in ROC have made strides in vaccination coverage but continued donor support from partner organizations must continue for additional substantial progress ${ }^{370}$. Unsurprisingly, donor support might very well be contingent on vital governance capabilities, many of which are enhanced at local levels and perhaps driven continually towards improvement in order to negotiate ongoing partnerships. Also in 2007, UNICEF collaborated with partners at the local, national and international levels to intervene in areas of health, nutrition and water, sanitation and hygiene (WASH). A key area of focused intervention was an "expanded programme on immunization (EPI)" in a handful of districts to increase coverage of the D.T.P. and measles, and polio vaccine, as well as vitamin A

\footnotetext{
368 Omar Azfar, Jeffrey Livingston, and Patrick Meagher, “Decentralization in Uganda," Decentralization and Local Governance in Developing Countries: A Comparative Perspective, $2006,223$. 369 Diamond, "The Rule of Law versus the Big Man 1," 261.

370 L. Arevshatian et al., "An Evaluation of Infant Immunization in Africa: Is a Transformation in Progress?," Bulletin of the World Health Organization 85 (2007): 455.
} 
supplementation in children and post-partum women ${ }^{371}$. The intervention campaign by UNICEF included plans to expand vaccination programs and care was taken to incorporate the effort within the "relevant ministries" as their capacity allows ${ }^{372}$. The readiness of international partners to collaborate at the local and regional levels in ROC indicates confidence in local and regional governance capacity and autonomy to deliver on predetermined targets. International organizations that are allowed to collaborate directly with decentralized localities might have more control over a program, its implementation, buy in or trust from communities and overall success. Decentralization seems a likely first step that allows other institutional drivers (like international partnerships, funding, performance-based funding, and cultural change) to get a foothold that also ultimately impact the indirect mechanisms that alleviate the proximate mechanisms of infant mortality.

The Narrative: Part II International Partnerships

A case study in Tanzania, Sirili et al. (2019) illustrates how the government implemented a series of reforms that paved the way for health sector PPP collaboration over time to address the medical professional deficit: in 1991 an amendment to the private hospitals regulation act allowed for the private ownership of health facilities; in 1994 proposals for health sector reforms were explored; by 1999 a National Education policy expanded science programs in secondary schools, in 2005 a Higher Education Students' Loans Board and the

\footnotetext{
371 UNICEF, “UNICEF Humanitarian Action Report 2008 Republic of the Congo Brazzaville," 3.
}

372 UNICEF, 4. 
Tanzanian commission for Universities was established; in 2009 the government reaffirmed commitment to PPPs with the endorsement of Public Private Partnership Policy that led to the 2010 enactment of the Public Private partnership act ${ }^{373}$. The case study of Tanzania is used as an example of a parallel initial experience of a LMICs African country establishing reforms and a private health care sector with PPPs as a response to national constraints. Similarly, Congo's private health sector was established roughly the same time as Tanzania's and it incorporates international partnerships as part of response to inadequate health care.

Analyzing the Narrative: International Partnerships

International partnerships seem to have influenced the indirect mechanisms that alleviate the proximate physiological mechanisms of infant mortality like delivery, community interventions, preventative actions, primary care, vaccines, and improved health protocols. From preconception through postnatal care, all phases of the chronology of care are marked by partnerships that impact health outcomes for infants in ROC. The partnerships suggest a light hand so to speak on behalf of the government to permit collaboration (international and domestic) through partnership, even a willingness to redistribute power through decentralization. International partners use their influence and encourage identified health outcomes through "increasingly dense interactions" in addition to funds and technical

373 Sirili et al., 6. 
expertise to realize stated objectives ${ }^{374}$. These intensified relationships provide opportunities to share international norms, standards, and expectations; sometimes implemented through performance based funding of projects and activities ${ }^{375}$. International partnerships result in more connectivity, access to funds, and improvement in health care systems that improve the IMR. Examples from ROC demonstrate the various means by which public private partnerships intersect to mitigate infant mortality.

International partnerships do not need to be at odds with broader government health goals, they can overlap as force multipliers and they can also promote their own specific agendas. Broadly though, international partnerships help the government achieve stated health outcomes, sometimes at the national level and frequently at regional and local levels. Between 2005 and 2012, maternal mortality decreased by over $45 \%$ due to improved healthcare and better family planning programs ${ }^{376}$. A UNICEF specialist in ROC says that the international partnership "in accordance with its commitments, the government, with the participation of stakeholders, will continue its efforts to implement additional measures to combat preventable deaths of children and mothers" 377.

\footnotetext{
374 Gunnar Folke Schuppert, “Partnerships," The SAGE Handbook of Governance. University of California, Berkeley: SAGE, 2011, 287-88.

375 Diamond, 255.

376 Charles Akena, "Maternal Mortality down Sharply in Congo," The New Humanitarian, January 21, 2013, http://www.thenewhumanitarian.org/report/97300/maternal-mortality-down-sharplycongo.

377 Akena.
} 
Since 2009, maternal mortality was made a health priority when the president committed to the Africa Union in an effort to ensure that women do not die in childbirth, and he hoped to do so through partnerships with UNICEF, the WHO, the World Bank, UNFPA (who helped clarify the "national roadmap" for achieving CARMMA- an international mission in Africa) ${ }^{378}$. CARMMA (Campaign for the Accelerated Reduction of Maternal Mortality) was formulated "around a coalition that includes government ministries, parliamentarians, the First Lady, civil society and the media, as well as UN agencies" 379 . The partnerships strengthen the Congo's leadership capacity to support improved health services and outcomes.

Not coincidentally, the Observatory on Maternal and Newborn Mortality created in 2010 in ROC improved maternal mortality according to the Ministry of Health ${ }^{380}$. The Observatory functions to emphasize family planning, Caesarean sections, and prevention of obstetric fistula. Government policies, multiple partners and NGOs were instrumental in the decline in IMR ${ }^{381}$. International partnerships' working in conjunction with Congo build strategies to increase education regarding the importance of maternal and child health care. Education and guidance by sector specialists facilitate enhanced family planning. Family planning encourages the prevention of actions and attitudes that limit the success of healthy pregnancy, delivery and postnatal care. The partnerships allow best practices to be integrated

\footnotetext{
378 Akena.

379 UNFPA, “Momentum Building for Maternal Health in Republic of Congo," UNFPA News, 2010.

380 Akena, "Maternal Mortality down Sharply in Congo."

381 Akena.
} 
into lagging health care systems. Family planning messages are increasingly coming through quality engagement with primary care based on enhanced training of the importance of family planning for maternal and child health outcomes.

By 2013, 93\% of women received antenatal care in Congo ${ }^{382}$. There was greater access to primary care in Congo due to scales of efficiency: as more people experience greater quality of care, more people feel encouraged to seek primary care. As more people seek primary care for their needs, the cost of service goes down ${ }^{383}$. Similarly, as more people sought primary care more vaccinations were available and they cost less ${ }^{384}$. Vaccinations are part of a healthy pregnancy, and in turn healthy babies.

Pregnant women in Congo who have received at least two tetanus toxoid containing vaccine (TTCV) doses during their last birth are considered to have "protection at birth" (PAB) ${ }^{385}$. A WHO-UNICEF mathematical model demonstrated in $2000,67 \%$ of pregnant women were considered to have $\mathrm{PAB}$, while in 2013 83\% of pregnant women were estimated to have $\mathrm{PAB}^{386}$. Globally, due to specific work "towards maternal and neonatal tetanus elimination (MNTE), neonatal mortality from tetanus has declined an estimated 94\% between 1988 and 2013"387. A focus of The World

\footnotetext{
382 Akena.

383 Cordaid, "Healthcare Approach Successful in Congo Brazzaville."

384 Cordaid.

385 Ridpath et al., "Progress towards Achieving and Maintaining Maternal and Neonatal Tetanus Elimination in the African Region."

386 Ridpath et al., Table 3.

387 Ridpath et al., "Progress towards Achieving and Maintaining Maternal and Neonatal Tetanus Elimination in the African Region."
} 
Health Assembly in 1989 was to eliminate neonatal tetanus globally by 1995 through partnerships with the WHO, UNICEF, and UNFPA to issue recommendations to achieve certain standards of routine care in pregnant women, particularly in Africa $^{388}$. In addition, the public private partnerships from the Polio Eradication Initiative, the Measles Partnership, and GAVI Alliance and other organizations are also revolutionizing vaccination in Africa ${ }^{389}$. International partnerships that offer technical advice to countries in crisis conditions can facilitate best practices when there might be little or no leadership from national governments. Country factors that limit health services like "civil unrest, lack of human resources within health ministries, limited funding for routine immunization services, and competition for staff time among individuals involved in polio and measles supplementary immunization activities"390 can be overcome through public private partnerships. For example, international partnership and funding from UNICEF between 2001 and 2005 was highlighted as "unprecedented" in targeting maternal and neonatal tetanus in Africa but it was considered necessary as some countries had limited capacity to address all vaccination needs ${ }^{391}$.

\section{The Narrative: Part III Funding}

Funding, frequently a companion of international partnerships, seems a likely institutional driver of consequence shaping indirect mechanisms that alleviate

\footnotetext{
388 Ridpath et al.

389 Arevshatian et al., “An Evaluation of Infant Immunization in Africa," 455.

390 Arevshatian et al., 450.

391 Arevshatian et al., 453.
} 
mechanisms of infant mortality in ROC. More obvious perhaps than decentralization, international partnerships, and/or performance-based funding, funding is what every underfunded health department wants. The list of competing needs is longer than available funds in most countries and ROC is no different.

Over ten years, from 2003 to 2013 , the maternal mortality rate dropped by $50 \% 392$ even though in 2005 there were still too few necessities (like forceps and beds) in health facilities according to one gynecologist ${ }^{393}$. But, by 2013 the difference was remarkable and credit is given to the government and presidential decree to dedicate financial resources towards health and free Caesareans that heretofore were prohibitively expensive ${ }^{394}$. This helped pregnant women who were previously left to die "on the table" if they were unable to pay ${ }^{395}$. The presidential decree created a path for the poorest of the poor to have access to life saving care in childbirth. This decree, nevertheless, comes about seven years after the Maputo Declaration in 2006, which emphasized a framework for improved access to sexual and reproductive health (SRH) for women in Africa, of which ROC participated ${ }^{396}$. Also in 2006 The United Nations Population Fund (UNFPA) contributed to neglected regions to ensure gains in health service delivery. Health care in peri-urban and

\footnotetext{
392 Jina Moore, "In Republic of Congo, a Revolution in Maternal Health," Al Jazeera America, 2013, https://www.google.com/search?client=safari\&rls=en\&q=in+republic+of+congo,+a+revolution+in+ maternal+health\&ie=UTF-8\&oe=UTF-8.

393 Moore.

394 Moore.

395 Moore.

396 UNFPA, "New Push to Promote Women's Health in Africa," 2006, /press/new-push-promotewomens-health-africa.
} 
rural areas was demonstrably poorer than in urban areas where the majority of the population lived. UNFPA worked with the government to build capacity in health centers that were underserved ${ }^{397}$. Regions as well as health sectors were overlooked and neglected. In 2008 a Congo Health Sector Services Development Project of The World Bank committed to release $\$ 40$ million of international development assistance to help mitigate communicable diseases, improve child health, and support population and reproductive health to focus on preventable causes of death ${ }^{398}$. At the time, it was estimated that between sixty and ninety percent of morbidity in ROC was due to preventable "infectious diseases, and maternal and child illnesses" 399 so The World Bank concentrated efforts in areas that would address the critical sectors. Funding (from the Maternal Health Trust Fund and partnership established by the G8 in Canada) was also an essential component to support "rapid improvement in addressing maternal and child health" in ROC by assisting the under served population at health facilities during delivery ${ }^{400}$. In 2013, The World Bank approved an additional $\$ 20$ million in funding for maternal and child health services to reach 86 percent of the population in addition to the $\$ 100$ million government commitment towards universal

\footnotetext{
397 UNFPA, “Momentum Building for Maternal Health in Republic of Congo.”

398 The World The World Bank, “Congo - Health Sector Services Development Project" (The World Bank, March 12, 2008),

http://documents.worldbank.org/curated/en/400941468247240552/Congo-Health-SectorServices-Development-Project.

399 The World Bank, 1.

400 UNFPA, "Momentum Building for Maternal Health in Republic of Congo."
} 
health ${ }^{401402}$. As part of a health-strengthening project, The World Bank through its International Development Association (IDA) wanted to increase services to women and children in support of the health related Millennium Development Goals (MDGs) 1, 4, 5 and 6, i.e. "reduce malnutrition, child mortality, communicable diseases (HIV/AIDS, TB) and improve overall maternal health" ${ }^{\prime 403}$. To achieve the established goals, the IDA project was intent on "building local capacity and promoting partnerships with local civil society" as well as strengthening the capacity of management ${ }^{404}$. The World Bank acknowledged that there were few donors for the health sector in ROC and that was why the project with IDA was essential to prevent further "catastrophic health expenses" for the impoverished ${ }^{405}$. Some of the funding for the IDA project was intended, at the request of the Ministry of Health and Population (MOHP) to support performance-based financing in the health sector ${ }^{406}$. The government of ROC identified that in order to improve health outcomes and meet the MDGs they would require a significant "capital investment twinned with technical assistance from the World Bank" 407.

\footnotetext{
401 World Bank, "WB to Help Republic of the Congo Provide Better Healthcare for Nearly 1.5 Million Mothers, Children," Text/HTML, World Bank, December 20, 2013, https://www.worldbank.org/en/news/press-release/2013/12/20/wb-congo-healthcare-motherschildren.

402 World Bank, "Republic of Congo: Health System Strengthening Project II (PDSS II)," November 25, 2013, 8, http://documents.worldbank.org/curated/en/236521468025764060/pdf/810090PAD0P143010Bo x379877B000U0090.pdf.

403 World Bank, 7.

${ }^{404}$ World Bank, 7.

405 World Bank, 7.

406 World Bank, 7.

407 World Bank, 8.
} 
Analyzing the Narrative: Funding

Funding, often through partnerships, strengthens the indirect mechanisms (for improved delivery, primary care, CHWs, improved health protocols, and preventative actions) that alleviate the proximate physiological mechanisms of infant mortality. In the absence of adequate social expenditure, funding from multiple streams can make a substantial impact on health outcomes in ROC, particularly if allocated well at the local level, especially through public private partnerships and/or performance-based funding. Whether from the central government, foreign aid, or partnerships, increased spending can provide for the barest of necessities in maternity wards as well as for sophisticated vaccination programs.

The Narrative: Part IV Performance-based funding Performance-based funding is the fourth institutional driver of change that shape the indirect mechanisms that alleviate the proximate mechanisms of infant mortality. It certainly falls under the broader previous category of funding as an institutional driver of change but it merits its own discussion because of the specificity associated with it. The focus is less on the availability of funds but rather how the funds are used. Performance-based funding (PBF) incentivizes desired behavior and health outcomes by giving money to organizations that achieve specified targets as identified. Performance-based funding can initiate within an organization, to drive specific behavior, or it can be initiated outside of an 
organization in order to shape said organization's outcomes. If, for instance, the desired goal is universal vaccination coverage for all infants born in a facility then funding for a health facility will be based on an organization's ability to achieve those specific results. Obviously, driving particular patterns of care can come at the expense of other equally important tasks. Like in the old adage, what gets measured gets improved. In crisis countries, where health systems are underperforming and shortages are the norm, following established health protocols and mindfulness in the quotidian care can improve health outcomes markedly. PBF encourages focused behavior in order to deliver specific results in dire conditions. PBF works to make health centers and health providers more accountable for specific health outcomes by remunerating organizations and individuals based on established criteria. PBF is often a partnership between the national government, international organizations, local health facilities, local government and patients that allow health professionals to be compensated based on quality care. Evidence points out that PBF in lowincome countries demonstrated "increased service coverage and improved service quality for maternal and child health services" 408 .

In the 1990s there was a movement in western countries to improve efficiency and accountability in public organizations. This movement was called New Public Management (NPM) and it drove individual and organization behavior by financial

\footnotetext{
408 World Bank, "Performance-Based Financing for Health in the Republic of Congo: Impact Evaluation Concept Note," 2014, 3, http://microdata.worldbank.org/index.php/catalog/2844/download/40107.
} 
incentives called performance-based funding ${ }^{409}$. In the case of performance-based funding for health, contract programs are typically between local level administration and health care providers; with the administration establishing contracts based on measurable health outcomes ${ }^{410}$. This differs from traditional financing because "agencies pay subsidies for health service achievements, such as a child fully immunized or childbirth conducted in a health facility"411. Performancebased funding has succeeded, even in countries considered to be "failed states" like the Democratic Republic of Congo, in reducing infant and maternal mortality ${ }^{412}$ because quality and efficiency improved in regions where it was implemented.

Analyzing the Narrative: Performance-based funding

Performance-based funding shapes the indirect health mechanisms like vaccinations and delivery, and indirect social and policy mechanisms like health protocols, community health workers, preventative actions, and community interventions in ROC. A "PBF pre-pilot" in the Republic of Congo in 2012, in coordination with the World Bank, demonstrated "substantial increases in service delivery during the first 12 months of curative consultations, assisted deliveries and tetanus toxoid (all are well over 10\% increase between 2011-2012. In addition,

\footnotetext{
${ }^{409}$ Amelia Compagni and Fabrizio Tediosi, "Implementing Performance-Based Funding for Health Research: When Governance and Procedural Fairness Matter," Public Administration 90, no. 2 (2012): 313, https://doi.org/10.1111/j.1467-9299.2011.01965.x.

410 Robert Soeters et al., "Performance-Based Financing Experiment Improved Health Care in the Democratic Republic of Congo," Health Affairs (Project Hope) 30, no. 8 (2011): 1518, https://doi.org/10.1377/hlthaff.2009.0019.

411 Soeters et al., 1518.

412 Soeters et al., 1519.
} 
quality of care has increased substantially in health facilities participating in the pre-pilot"413414.

Some examples of successful PBF in Congo focus on leveling up in health protocols. This emphasis is seen in an international partnership between Cordaid and ROC. Cordaid had successfully introduced PBF in several other African countries too like Rwanda and Zimbabwe. Based on the success of PBF in improving health services in other developing countries, Cordaid and Memisa Belgium partnered with Congo in Brazzaville to improve health services through performance based measurements like the number of assisted births, vaccinations, and consultations ${ }^{415}$. There was a focus on attention to detail, ensuring that women were encouraged to have assisted births, vaccinations were more consistently tracked, and that recommended number of consultations was conducted. Tracking consultations is especially important because pregnant women might not seek care due to financial reasons. Of the approximately $95 \%$ of pregnant women that do pay for a single pre-natal appointment in Congo, 15\% of those women do not return for additional recommended appointments ${ }^{416}$. Finding ways to incentivize, support and treat

\footnotetext{
413 World Bank, "Performance-Based Financing for Health in the Republic of Congo: Impact Evaluation Concept Note," 7.

${ }^{414}$ World Bank, "WB to Help Republic of the Congo Provide Better Healthcare for Nearly 1.5 Million Mothers, Children."

415 Cordaid, "Healthcare Approach Successful in Congo Brazzaville," 2.

416 Moore, "In Republic of Congo, a Revolution in Maternal Health."
} 
these under served pregnant women in Congo through PBF is an important driver of IMR change.

A pre-post study between 2012 and 2014 of PBF in ROC revealed statistically significant results on "curative visits, patient referral, children receiving vitamin A, HIV testing of pregnant women and assisted deliveries" 417 . The survey that was conducted for the study also found that there were "significant improvements in quality of services regarding the availability of medicines, perceived quality of care, hygiene of health facilities and being respected at the reception desk" 418 . Financed by the World Bank and supervised by ROC's Ministry of Health and Population, PBF incentivized selected Mother and Child Health (MCH) services ${ }^{419}$.

\section{The Narrative: Part V Cultural Change}

Cultural change is the last institutional driver identified as potentially having an impact on the indirect mechanisms that alleviate the proximate physiological mechanisms of infant mortality. Culture plays a huge part of health care for mothers and infants in ROC. Some women still give birth at home and are cared for by a traditional birth attendant, or "matron". This practice is essentially considered an "unattended" birth because conditions are not optimal for healthy outcomes for mother or the infant. Changing the culture around what type of care is acceptable

\footnotetext{
417 Wu Zeng et al., "Evaluation of Results-Based Financing in the Republic of the Congo: A Comparison Group Pre-Post Study," Health Policy and Planning 33, no. 3 (April 1, 2018): 392, https://doi.org/10.1093/heapol/czx195.

418 Zeng et al., 392 .

419 Zeng et al., 393.
} 
and desirable for a pregnant mother in ROC requires time, education and experience. Many women were mistrustful of health care because in the past they heard from others that health facility care was poor, expensive and/or it resulted in the death of the mother or the infant. Rightly so, women were concerned about the quality of care available in health systems in ROC- it has been substandard and the message was shared among pregnant women.

One instance of cultural change started in 1996 when the ROC government started a review of health-sector reforms initiated in the late 1980s that aimed to improve the nutritional status of children under the age of five ${ }^{420}$. Over a ten-year period, the government review found that stunting and wasting in children increased from $12.4 \%$ to $16.7 \%$ and from $3.2 \%$ to $5.1 \%$ respectively ${ }^{421}$. As a result, the Ministry of Health, the Ministry of Scientific Research (along with donors, NGOs and local representatives), and the Faculty of Sciences initiated a program to improve the child growth and development sector of primary care to focus on "perceptions, beliefs, and behaviors of both mothers and health professionals" regarding child development ${ }^{422}$. The program involved research to facilitate health workers in their interactions with new mothers. New mothers and health care workers had different ways to communicate if infants were thriving or not. Through the program, health

\footnotetext{
420 Félicité Tchibindat et al., "Bringing Together Viewpoints of Mothers and Health Workers to Enhance Monitoring and Promotion of Growth and Development of Children: A Case Study from the Republic of Congo," Journal of Health, Population and Nutrition 22, no. 1 (2004): 60.

421 Tchibindat et al., 60.

422 Tchibindat et al., 60 .
} 
care workers collaborated with new mothers to identify indicators that suggested health and wellness. For instance, health workers used "anthropometric indicators, such as weight and height" to demonstrate infant thriving while new mothers used indicators like "movement, thinking, physical growth, and interaction with people and objects" to demonstrate infant vitality ${ }^{423}$. Improving cultural communication between new mothers and health care workers was viewed as essential in order to strengthen trust, encourage mothers to engage more with health workers, and improve health outcomes for infants.

\section{Analyzing the Narrative: Cultural change}

Cultural change can impact the indirect medical mechanisms (like breastfeeding, delivery, primary care and vaccines) as well as the indirect social and policy mechanisms (like community health workers, health education, community interventions, women's health groups, preventative actions, and improved health protocols) are influenced by culture. Improved cultural understandings between health care workers and new mothers builds trust and more engagement. In the instance of improving health-monitoring indicators in ROC, health workers focused on cultural adaptation in their clinical practice to address short falls in service delivery. The transformation of cultural attitudes both in society and in health care an opportunities to shape the indirect medical, and social and policy mechanisms that alleviate the proximate physiological mechanisms of infant mortality.

423 Tchibindat et al., 61-62. 
The type of assistance at delivery, particularly for the disenfranchised in rural regions is critical. Typically, a home birth with a "matron, an untrained traditional birth attendant" means care is not in line with recommended health protocols for delivery: "in some parts of the bush, it's archaic. When they need to weigh the baby, they put it in a wrap and rest it on the same scale they use for meat" 424 . As more mothers' deliver in health facilities they have an opportunity to encounter improved, quality health services. Improving the knowledge and quality of care that community health workers are able to provide in rural communities improves health outcomes for the women who do deliver at home, or have no other choice than to deliver at home. The intervention on behalf of a trained community health worker or midwife at delivery has the opportunity to save lives through quality care, and low technology practices supported by the medical community, instead of relying on traditional practices like using herbal medicines and unhygienic practices $^{425}$. Changing the culture from relying solely on traditional matrons often comes from more education and exposure to improved health practices that deliver better health outcomes.

There has also been a slow cultural change regarding HIV in Congo. HIV has been a taboo subject and people live in denial and do not want to discuss it. The Republic of Congo experienced HIV early on as one of the first countries in Africa to be impacted

\footnotetext{
424 Moore, "In Republic of Congo, a Revolution in Maternal Health." 425 Moore.
} 
(in $20014.4 \%$ of adults in ROC were HIV positive) ${ }^{426}$. The IMR of babies born to HIV-infected mothers was more than 260 per 1,000 or, " 6.3 times higher than that among children born to HIV-uninfected mothers ${ }^{427}$. Mitigating the prevalence of HIV in infants is one way infant mortality improved. HIV/AIDS "remains highly stigmatized" in Congo as the culture is one of avoidance and denial in discussing the epidemic ${ }^{428}$. Diagnosis was often under reported due to the stigma. Infant death in a study from 2001 shows that $51.1 \%$ of deaths were from neonatal deaths, $6.7 \%$ from malaria, 4.5\% from measles, 7.5\% from AIDS, 11.2\% from respiratory diseases, 9\% from digestive issues, $3.4 \%$ from infectious diseases, $3.4 \%$ from anemia, $.7 \%$ from accidents, .7\% from other noninfectious disease, .7\% indeterminate, and 1.1\% malnutrition ${ }^{429}$. This study from 2001 shows that improvement in neonatal deaths would substantially improve infant mortality and that treating malaria, measles and respiratory diseases could improve infant mortality even if drugs were not available to prevent the transmission of HIV from mother to infant in pregnancy or through breastfeeding. Highly active antiretroviral therapy (HAART) has demonstrated effectiveness in treating HIV infants but it is important for the infants and mothers to be tested for HIV systematically so they can be given the "antiretroviral

\footnotetext{
${ }^{426}$ Camille Lallemant et al., "Impact of HIV/Aids on Child Mortality before the Highly Active Antiretroviral Therapy Era: A Study in Pointe-Noire, Republic of Congo," Journal of Tropical Medicine 2010 (2010): 1, https://doi.org/10.1155/2010/897176.

427 Lallemant et al., 1-3.

428 Lallemant et al., 3.

${ }^{429}$ Lallemant et al., 4.
} 
regime" 430 . The provision of HAART in infants could reduce infant mortality by $76 \%$ "within the first year of life independently of their clinical or immunological condition" ${ }^{\prime 31432}$. The cultural component associated with HIV diagnosis has prevented mothers and infants from being tested. Neglecting the possible life saving antiretroviral treatment contributes to infant mortality but with coordinated antenatal and postnatal quality care, the administration of these drugs save lives. Changing the culture around HIV testing as part of routine care allows for, not only discussion of prevention strategies, but intervention when indicated. Cultural change is slow but it has allowed more pregnant women to embrace and seek out modern health care.

\section{f. Initial statement of "strategic governance" findings}

This chapter has identified five likely drivers of the IMR decline in ROC. They are decentralization, international partnerships, funding, performance-based funding, and cultural change. As discussed, they can reasonably be linked to the indirect medical, and indirect social and policy mechanisms that alleviate the proximate physiological mechanisms of infant mortality in spite of macro structural weakness. These five factors are both consistently present as well as demonstrably relevant to the circumstances under which the indirect mechanisms were shaped. They reflect a

\footnotetext{
430 Lallemant et al., 5 .

${ }^{431}$ Camille Lallemant et al., "Impact of HIV/Aids on Child Mortality before the Highly Active Antiretroviral Therapy Era: A Study in Pointe-Noire, Republic of Congo," Journal of Tropical Medicine 2010 (2010): 5, https://doi.org/10.1155/2010/897176.

432 Avy Violari et al., "Early Antiretroviral Therapy and Mortality among HIV-Infected Infants," New England Journal of Medicine 359, no. 21 (2008): 2233-2244.
} 
set of untheorized factors which form the basis for further thesis building under the concept that has been defined here as "strategic governance". These five drivers of institutional change lead us to consider three points regarding how they might relate to strategic governance. First, each is individually and mutually enforcing the other. They involve institutions, policy and norms. Second, as some of the institutional drivers are implemented from within ROC, it suggests that there are endogenous drivers of change, even in crisis conditions, as seen through decentralization and cultural change. Third, strategic governance might also involve a dependent phenomenon that encourages and supports exogenous partnerships, funding, and performance-based funding.

The institutional drivers of decentralization, partnerships, funding, performancebased funding, and culture each (and collectively) mitigate against underperforming macro-structural forces, i.e., stunted democracy, poor governance and sluggish economic growth. For instance, decentralization can (intentionally or not) circumvent some of the dysfunction prevalent in undemocratic (or underperforming) regimes by collaborating with regional or local governments. Decentralization can minimize interaction with poor governance at the national level and can force specific accountability at lower levels of government through monitoring. Decentralization also reduces risk of capital flight because there is potentially more oversight from funding partners invested for specific health outcomes. By redirecting capital to specific health projects, the negative effects of an underperforming economy on health can be diminished. 
In the case of international partnerships, the engagement with multiple stakeholders brings specific expertise to maternal and infant care that benefits specific ROC health goals. International partnerships, in particular, bring subject matter expertise, funding, and international norms to bear that might get overlooked in crisis conditions. The interaction between international partners and local governments can introduce accountability, transparency (where lacking) and efficiencies of scale through training and sharing best practices. Where undemocratic practices are commonplace, international partnerships can introduce expectations of equity and accountability. Additionally, international partnerships introduce and/or reinforce good governance practices that can inform and instruct poor governance practices. International partnerships also provide necessary funding so regardless of economic feasibility in ROC, resources are made available to achieve specific health targets that reduce IMR.

As we have seen, funding can take the place of public funding (to a degree) that has been misappropriated through channels at the national level. The funding does not lessen the pillage but it can bypass some of the more porous channels at the national level to target specific local programs, trainings, services, and/or equipment. Funding for vaccination programs, and health education for instance, can happen regardless of the economic outlook of the country. Even if the economy is underperforming, funding from international partners for targeted health outcomes can bolster the capacity of health workers by providing needed materials, i.e., vaccinations, hospital beds, forceps, updated training. 
Performance-based funding works directly against poor governance by incentivizing specific behavior to achieve certain health outcomes and better governance within health care systems. The focused and agreed upon actions of health workers and health systems, when properly structured through performance-based funding, can supersede poor governance in some health care environments.

Cultural change seems less connected or linked with mitigating macro pessimistic structural factors but it is still very powerful in its own right as a force to improve the IMR in Congo. Decentralization and cultural change are primarily internally (or domestically) driven while international partnerships, performance-based funding, and funding are roughly external institutional drivers that impact the indirect mechanisms that alleviate the proximate physiological mechanisms of infant mortality. 


\section{Chapter 5: Republic of Yemen}

This research will thus proceed in the following fashion: a) country background of pre-colonial, colonial, Cold War and post-Cold War history, especially as relevant to IMR; b) description of macro-structural weaknesses of democracy, governance, and economic growth; c) a description of IMR decline is described using surveys from two different time periods; d) description of indirect mechanisms that alleviate the proximate causes of infant mortality; e) case study analytic narrative development and analyzing the narrative; f) initial statement of "strategic governance".

\section{a. Country background}

\section{Pre-Colonial}

The pre-colonial history of the current day Republic of Yemen foretells its future: a region at odds with itself and the site of proxy wars for international greed and creed. Situated on the southwest corner of the Arabian Peninsula, Yemen has long been in the path of global routes connecting east and west. Yemen also has 112 islands situated in the Indian Ocean and the Red Sea. As such, Yemen has firmly been in the way of ancient competing agendas, rulers, and civilizations. Inhabited since the earliest times, this area has experienced intermittent prosperity but more often than not global trends shift the balance out of favor. Once a trade route between Asia, Africa and Europe and greater Mediterranean area for frankincense, the market was dismantled essentially by the first century when the Romans 
learned how to sail to India and the Far East ${ }^{433}$. The new sea going trade routes left Yemen behind until the seventh century when people in the region started to convert to Islam. The connection to Islam provided connections to other parts of the world through the arts and sciences. Local dynasties and foreign empires ruled Yemen variously and by the late ninth century, one local dynasty, the Zaydi Imamate, was founded and continued to influence the northern region of Yemen for almost one thousand years ${ }^{434}$.

\section{Colonial}

In the sixteenth century, Yemen emerged again at the crossroads of global events when foreign powers (the Portuguese, Dutch, British, Italian, French, American, Turkish and Omani empires) wanted control of Yemen and neighboring regions for strategic reasons: the Portuguese in 1507 took over the island of Soqotra, in 1938 the British subjugated the port of Aden, and the Ottoman Turks twice conquered the country between 1538 and 1636, and again in 1849 and $1918^{435}$. Coffee plants were important indigenous crops and drinking coffee was popularized during the Ottoman's occupation in the sixteenth century; however, by the end of the seventeenth century the Portuguese and Dutch undermined the coffee monopoly in Yemen by reproducing crops in their colonial territories of Java and Brazil ${ }^{436}$. While

\footnotetext{
433 Marta Colburn, The Dynamic of Development and Democratization in Yemen (Bonn: FriedrichEbert-Stiftung, 2002), 11.

434 Colburn, 12.

435 Colburn, 12.

436 Colburn, The Dynamic of Development and Democratization in Yemen, 13.
} 
foreigners battled over the centuries for sovereignty, local tribes and leaders opposed reigning authority.

\section{Cold War}

With the end of World War II and the breakdown of the Ottoman Empire the Zaydi Imam exerted more authority. From 1901 to 1948 Imam Yahya a proponent of isolationist, conservative culture in northern Yemen ruled and it continued with his successor (son) Ahmed from 1948 until 1962437. The revolution in 1962 created the Yemen Arab Republic (YAR) in the north but civil war ensued until 1970 between republicans and the pro-Imam royalists ${ }^{438}$. Meanwhile throughout the early part of the twentieth century, further south in Aden, the British incrementally exerted control through treaties negotiated with locals as they expanded control into South Yemen. The occupied Aden Colony was formalized in 1937 in addition to both Eastern and Western Protectorates until a revolution in 1967 (although the fighting had already begun in 1963 when the National Liberation Front (NFL) was founded). The British then left the Federation of South Arabia and handed power to the National Liberation Front (NLF) of the south who then formed the People's Republic of South Yemen ${ }^{49}$. By 1970, the People's Republic of South Yemen became the People's Democratic Republic of Yemen and was formalized as a socialist regime ${ }^{440}$.

\footnotetext{
437 Colburn, 13.

438 Helen Lackner, Yemen in Crisis: Road to War (Verso Books, 2019).

439 Lackner.

440 Colburn, The Dynamic of Development and Democratization in Yemen, 13.
} 
Unification of the two Yemens did not happen for a couple of decades but still the period was not without conflict (most notably in 1971, 1972, 1978, 1979, and 1988). While the Yemen Arab Republic (YAR) in the north and People's Democratic Republic of Yemen (PDRY) in the south were newly established in close proximity to each other, the traditions they followed were vastly different. YAR came out of an Islamic tradition and accordingly continued with "Islamic, tribal, civil and military" type rule ${ }^{441}$. At this time, patronage emerged as a dominant trait of the regime and funds from oil exports ensured that leader Saleh had loyalty from disparate groups that wanted the public funds he controlled ${ }^{442}$. International donors funded public projects, and global aid flowed to YAR signaling support for the free market economy contrasted with the socialist PDRY that was encouraged primarily by aid from the East ${ }^{443}$. The lack of public investment and the nationalization of property represented a continued and significant divergence from YAR in the north ${ }^{444}$. Hastening the unification of the two Yemens was the collapse of the Soviet Union as well as internal political, social and economic disputes ${ }^{445}$. One such dispute was over the discovery of oil on the borders of YAR and PDRY and how to prevent a Saudi Arabian takeover. Unification seemed to be one way to keep the two rival republics

\footnotetext{
441 Sheila Carapico, Civil Society in Yemen the Political Economy of Activism in Modern Arabia, Cambridge Middle East Studies 9 (Cambridge, U.K. ; New York: Cambridge University Press, 1998), 20.

442 Lackner, Yemen in Crisis, 101-2.

443 Carapico, Civil Society in Yemen the Political Economy of Activism in Modern Arabia, 20.

444 Carapico, 20.

445 Colburn, The Dynamic of Development and Democratization in Yemen, 13-14.
} 
from losing out to a stronger rivaling neighbor country ${ }^{446}$. The intervention of international interests in a unified Yemen that could create a booming oil export economy also helped clinch deals for access to southern Yemen ${ }^{447}$.

\section{Post-Cold War}

The Republic of Yemen was created on May 22, 1990 with the unification of the Peoples Democratic Republic of Yemen in the south and the Yemen Arab Republic to the north ${ }^{448}$. Ali Abdullah Saleh (formerly the president of the Yemen Arab Republic) became the president, and Ali Salem al-Beedh (formerly the Secretary General of the Yemeni Socialist Party) was the vice-president. ROY was born out of two opposing governing styles; each with their own complicated history. After unification, there were challenges merging courts, public sectors, and militaries ${ }^{449}$. Parliamentary elections considered 'free and fair' were held in 1993 and 1997450, even so, the civil war ensued in 1994 with the Yemeni Socialist Party (YSP) failed secession attempt ${ }^{451}$. After the civil war, the constitution was changed to give the president more power through extended terms limits and the number of allowable terms extended ${ }^{452}$. In 1995 the government accepted IMF input and Structural Adjustment policies (SAP) to improve the economy, and by 1996 ROY introduced its

\footnotetext{
446 Lackner, Yemen in Crisis, 117.

447 Isa Blumi, Destroying Yemen: What Chaos in Arabia Tells Us about the World (Oakland, California: University of California Press, 2018), 149-52.

448 Lackner, Yemen in Crisis.

${ }^{449}$ Carapico, Civil Society in Yemen the Political Economy of Activism in Modern Arabia, 19.

450 Marta Colburn, The Republic of Yemen: Development Challenges in the 21st Century (London: Stacey International, 2002), 19.

451 Colburn, The Dynamic of Development and Democratization in Yemen, 39.

452 Lackner, Yemen in Crisis, xix.
} 
First Five-Year Economic Development Plan ${ }^{453}$. The SAP policies included increasing prices on oil products, increasing electricity tariffs, decreasing the budget deficit, lowering the exchange rate, increasing the interest rate, and the issuance of treasury bills $^{444}$. A second Five-Year Economic Development plan was put forth in 2001 to address ongoing shortfalls in the country.

Throughout the 1990s Yemen dealt with political pluralism and efforts at democratization but tribalism, economic stress and violence-marred progress ${ }^{455}$. In 1999 Ali Abdullah Saleh won the first presidential election and soon after contended with growing terrorist action in ROY with the attack on the USS Cole in the port of Aden and the French tanker Limburg by al-Qa'ida ${ }^{456}$. The US invasion of Iraq in 2003 also was an international complicating factor for ROY as they opposed sanctions against Saddam Hussein's regime and suffered a decrease in aid as a consequence ${ }^{457}$. The nearby fragile state of Somalia, and subsequent migrants crossing the Red sea into ROY was another global pressure for the government ${ }^{458}$. The World Bank recognized the dire situation in Yemen in 2002 and worked with the government to introduce a Poverty Reduction Strategy Paper (PRSP) for 2003 to 2005 to address escalating human and economic crisis; however growing discontent

\footnotetext{
453 Lackner, xix.

454 Nora Ann Colton, "Yemen: A Collapsed Economy," The Middle East Journal; Washington 64, no. 3 (Summer 2010): 418, http://dx.doi.org.proxy.lib.pdx.edu/10.3751/64.3.14.

455 Colburn, The Dynamic of Development and Democratization in Yemen, 14.

456 Lackner, Yemen in Crisis.

457 Lackner, 83.

458 Lackner, 83-84.
} 
led to further degradation of conditions. The first decade of the new millennium was no different. The Huthi wars (which initiated in the northern region of former YAR in the Sa'ada provice) from 2004 until a cease fire in 2010 disrupted the full integration of the former YAR and PDYR. The Huthis are considered fundamentalists and they are distinct, separate and not aligned with Sunni jihadi movements ${ }^{459}$. The Huthi movement initially came out of the marginalization of the Zaydi population by Saleh's government and influenced by the popular Zaydi cleric leader Husayn alHuthi ${ }^{460}$. Husayn refused to align his movement with Saleh's regime and in 2004 war erupted between government forces and the Huthis in the northern territory. This initial campaign led to Husayn's death and martyrdom and launched a series of wars that won the Huthis more territory. By 2011, the Huthi movement controlled parts of Amran province, western al-Jawf province, and by 2014 most of northern Yemen and all of Sana'a ${ }^{461}$.

By spring 2011 popular uprisings led to encampments ${ }^{462}$ in cities across ROY. This movement was called the "Change Revolution" 463 and it marked a culmination of anger at Saleh's regime. During this time, Saleh's forces fired upon protestors killing 45 people and injuring over 200- this violent attack caused some of his party

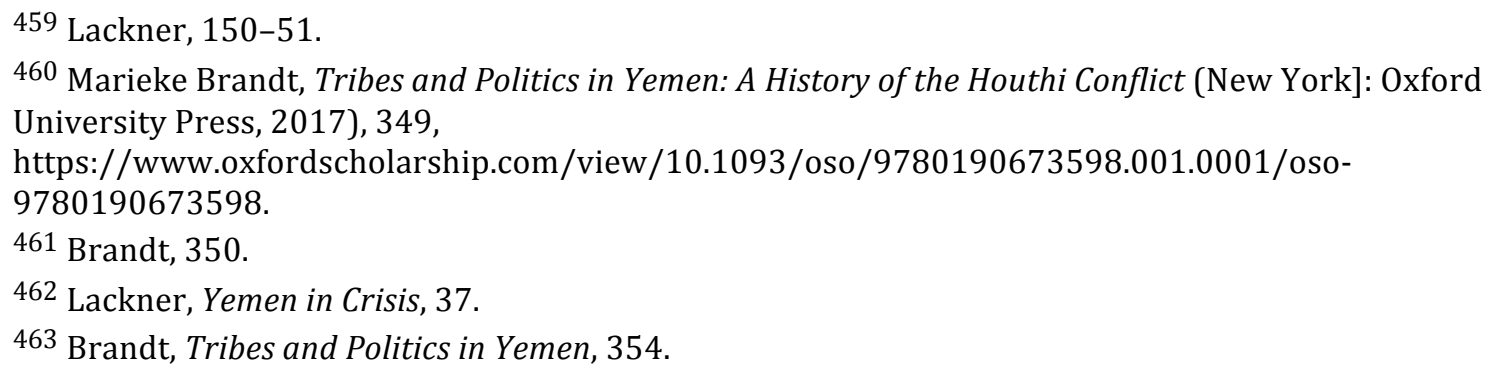


members to leave the party and it also caused a split in his military where one brigade vowed to defend the protestors ${ }^{464}$. While the government was opposing the Huthis in some regions, in an improbable union, other Huthis were "cooperating with Saleh elsewhere in the country" 465 . Seriously wounded in a mosque attack, Saleh was evacuated to Saudi Arabia for medical attention and that initiated a formal takeover by Abdu Rabbu Mansur Hadi (who was subsequently elected president for a two year transitional period) ${ }^{466}$. When Saleh returned to Yemen after his convalescence he extended his "secret" involvement with the Huthis in a push against the Hadi regime ${ }^{467}$. By 2014 the Huthi-Saleh alliance took over Amran and Sana'a further unsettling ROY, while at the same time Hadi was also trying to harness Huthi fighters to undermine forces against his transitional government ${ }^{468}$. Betrayal, deception and changing loyalties throughout the country strengthened the Huthis by the time they took over the capital city as Hadi's transitional regime became increasingly unpopular until the Peace and National Partnership Agreement (PNPA) was signed with UN involvement ${ }^{469}$. While the case study time frame that looks at improvement in IMR in ROY extends only to 2014, the complicated history of ROY continued with Machiavellian twists. Breakdowns in 2015 in efforts to draft a new constitution because of kidnappings, military maneuvers, and attacks against

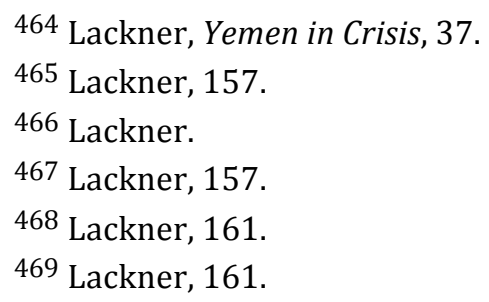


Hadi's personal security force led Hadi's and his government to resign and escape to Saudi Arabia ${ }^{470}$. Shortly thereafter, Hadi tried to reestablish a government in Aden with the help of Saudi military support but the Huthi-Saleh alliance initiated an attack to establish military prominence. The Saudi involvement in support of Hadi's failed transitional government turned a "political and humanitarian crisis into a catastrophe" 471 . Then, in February 2015 the Huthi campaign made a "Constitutional Declaration" that stated that nothing in the constitution could challenge their authority. The Huthi Supreme Revolutionary Committee (SRC) usurped the government and this new arrangement suited Saleh well ${ }^{472}$. It is thought that the former regional Huthi movement that came to ultimately takeover the entire country is proof of Saleh's maneuvering ${ }^{473}$. The Huthi leadership and military was essentially loyal to Saleh himself rather than the Huthi movement (which by this time was an amalgamation of political, tribal, and military fighters and little ideological coalescence) but Saleh had no allegiance to the Huthis except for that they were potentially fighting against any force that threatened a common interest in controlling the country ${ }^{474}$. Four months later, the Supreme Political Council (SPC) of Saleh's making, which replaced the Supreme Revolutionary Committee, established the Government of National Salvation ${ }^{475}$. Top institutional and

\footnotetext{
470 Lackner, 161.

471 Lackner, 287.

472 Lackner, 162.

473 Lackner, Yemen in Crisis, 162.

${ }^{474}$ Lackner, 162.

475 Lackner, 163.
} 
ministerial level positions of the SPC were held by Huthi and Saleh followers alike but always in an uneasy alliance that made effective rule impossible ${ }^{476}$. Still in early 2017 a Saudi-led coalition in support of "internationally recognized government forces" and the Huthi-Saleh leaders battle for supremacy ${ }^{477}$. Later in December of that year, the Huthis assassinated Saleh when they discovered he was leaving the coalition only to side with Saudi-led support of Hadi's "recognized" government. The initial Saleh and Hadi split created enormous tension and ignited already tense relations in Yemen between regions of the former YAR and PDYR.

b. Description of macro-structural weakness

\section{Democracy}

One of the macro-structural weaknesses in ROY is the failed state of the fledgling democracy. Democracy, understandably, struggles in ROY due to recurrent civil war and different historical traditions that challenge power consolidation. From unification, there was consensus for a democratization of the disparate practices coming out of different traditions. Democratization initially facilitated unification by combining two different "economic and political systems and actualized ancient concepts of 'the Yemen' and 'the Yemeni people'"478 but it was short lived. Despite efforts to forge a democracy at unification ROY it was plagued by intermittent civil wars, ulterior motives for power, access to resources and financial gain even to the

\footnotetext{
476 Lackner, 166.

477 Lackner, 289.

478 Colburn, The Dynamic of Development and Democratization in Yemen, 39.
} 
extent of harboring terrorists for political gain ${ }^{479}$. Even though some established ROY with democratic intentions, the actual institutions of the democracy were never really democratic or functioning ${ }^{480}$ as they were largely an extension of Saleh's elite gang of cronies, military, and convenient supporters. So the situation in ROY was flawed from the start, and it never recovered even though there were some notions of "democracy" it never materialized. USAID gives Yemen a liberal democracy score of .04 on a scale of 0 to $1^{481}$.

\section{Governance}

The macro-structural constraint of poor governance in ROY, as in ROC, undermines many natural opportunities for the country to succeed. Governance was not a priority during Saleh's rule as he wielded the government as a personal convenience for his elite dealings ${ }^{482}$. Standard operating procedure was much like it had been in YAR: "personal patronage", access to spoils from the oil sector, favors for favored family, military, tribes, and access to board positions for his chosen friends and implemented under "the veneer of democracy"483. Before Saleh died in 2017 his net worth was estimated between $\$ 32$ billion and $\$ 64$ billion dollars ${ }^{484}$.

\footnotetext{
${ }^{479}$ Sophia Dingli, "Is the Failed State Thesis Analytically Useful? The Case of Yemen," Politics 33, no. 2 (2013): 96, https://doi.org/10.1111/j.1467-9256.2012.01453.x. 
A combined governance score, comprised of the average of the voice and accountability score and the government effectiveness score from the worldwide governance indicators, is -1.01 on a scale of -2.5 to 2.5 from 1996 to 2014. USAID gives Yemen a score of .12 for government effectiveness on a scale of 0 to $1^{485}$.

\section{Economic growth}

The third macro-structural weakness is that Yemen has experienced a growing economic crisis for decades even though there was an average economic growth of $3.311 \%$ during this study's timeframe from 1996 to 2014 (compared to an average economic growth of $4.77 \%$ for 106 developing countries over the same time). Structural adjustment policies in the 1990s tried to create a stable environment for economic growth but ultimately it did not have the desired impact. A migration crisis, coupled with an under educated working class, wars, slowing of remittances, and a drop in foreign aid all contributed to Yemen's economic crisis. Prior to the 1990s, people from both the northern and southern regions of current Yemen went abroad to earn higher wages in Saudi Arabia. Workers would send remittances back home to help their families. In 1982, remittances were over $\$ 1$ billion to YAR, and remittances to PDRY were estimated to be more than half of GNP ${ }^{486}$. However, when Yemen sided with Iraq in the Gulf War in 1990, Yemenese workers no longer had the possibility to remain or work in Saudi Arabia. There was a flood of expatriated workers that returned to a unified Yemen with hardly any prospects for work, there

\footnotetext{
485 USAID, "Yemen, Journey to Self-Reliance: FY 2020 Country Roadmap."

486 Colburn, The Republic of Yemen, 17.
} 
ensued a corresponding drop in remittances. Foreign aid was cut back significantly as Yemen sided with Iraq, and oil prices dropped. In 1991, foreign aid dropped to approximately $\$ 191 \mathrm{~K}$ but increased again to $\$ 25$ million per year for the next three years ${ }^{487}$. The established economic conditions were poor from the time of unification and did not improve much over the next two decades even with different phases of structural adjustment. By 2010 , nearly $40 \%$ of the population was estimated to be unemployed and over $50 \%$ of the population living on less than $\$ 2$ a day ${ }^{488}$. The economy continued in shambles over the timeframe for this study and that is the backdrop from which the IMR reportedly improved greater than the average of other developing countries over the same time.

c. Description of IMR decline Considering the complicated history and considerable macro-structural weaknesses of ROY, this research considers the reduction in IMR against the backdrop of crisis and chaos. In 1996, the IMR in Yemen was 78.2 and by 2014 it was 35.1 according to globally aggregated data from the World Bank. In less than two decades Yemen more than halved the IMR under crisis conditions. Neighboring Saudi Arabia had an IMR of 23.4 in 1996 and 12.9 in 2014, Oman was 19.2 and 10 respectively. This Yemen case study explores longitudinal data from the Demographic Health Surveys

\footnotetext{
487 Colton, "Yemen," 416.

488 Colton, 418.
} 
(DHS) from $1997^{489}$ and $2013^{490}$ and other sources to find clues of how the IMR in Yemen dropped so significantly. Longitudinal data demonstrates where some change occurred and reveals improvement at a micro level (individual and community) even as the macro level (national) remains challenged. While there are improvements in Yemen overall during this time, it is important to note that significant differences persist between rural and urban communities in both data. See Appendix D. See Appendix E. However, there might be reason to be skeptical of the reported estimates from the World Bank and other sources. The estimates of IMR in Yemen range from 79.5 in 2010 from the CIA Factbook, Yemen as cited in Colton (2010) ${ }^{491}$. An earlier estimate of 82 deaths per 1,000 infant live births in 2003 as cited in a strategy paper on Yemen from the European Community ${ }^{492}$ with information gathered from the World Development Indicators database that conflicts with an IMR of 60.1 for the same year in the dataset used for this study. This mixed evidence suggests we need to treat cautiously the claim that ROY experiences a dramatic decline in its IMR in this period.

\footnotetext{
${ }^{489}$ Central Statistical Organizaton (CSO) and Macro International, "Yemen Demographic and Maternal and Child Health Survey 1997," November 1, 1998, https://dhsprogram.com/publications/publication-FR94-DHS-Final-Reports.cfm. 490 MOPHP/Yemen et al., "Yemen National Health and Demographic Survey 2013" (Rockville, Maryland, USA, 2015). 491 Colton, "Yemen," 418.

492 European Commission, "Yemen- European Community Strategy Paper for the Period 2007-2013," n.d., 32 .
} 
d. Description of indirect mechanisms that alleviate the proximate physiological causes of infant mortality

Between the first Demographic Health Survey in Republic of Yemen (DHS) in 1996 and the second DHS in 2013 a number of indirect mechanisms (both medical, and social and policy) that alleviate the proximate physiological mechanisms of infant mortality were improved as demonstrated through the chronology of care for women of childbearing age. The comparable data reveal changes over time and provides clues as to how the IMR improved substantially even in crisis conditions. Many of the improvements in health care are slight and are not universal, while other changes are considerable in the chronology of care that shape IMR.

\section{Preconception}

In defiance of the odds, data organized under the category of "preconception" phase of women's health care demonstrates improvement. Unlike available survey data from ROC, ROY survey data demonstrates that women gained knowledge that impacted choices and behavior impacting their health and the health of their eventual family. More women heard family planning messages on TV, contraceptive use increased, knowledge of family planning improved, the median age of first marriage grew, the age of mother at first birth was higher, birth intervals lengthened, the median age of first birth increased, the IMR based on birth order improved, and the ideal number of children desired decreased. This preconception phase made remarkable strides in preventing compromised health outcomes for 
women. By combating early and frequent pregnancy, the IMR improved, as there were fewer compromised pregnancies in the first place, and women were more physically capable and ready for pregnancy. This preconception phase reveals that significant change in IMR is in the control (agency) of women and families through preventative actions, health education, and not necessarily just at the primary care provider level.

For instance, a general trend in the preconception phase was access to more health information and knowledge. Educational messages from media reached more women. The number of women who were ever married to see a family planning message on TV grew substantially. Interestingly, there was a corresponding increase in contraceptive use over the same period of time.

The corresponding contraceptive use could have been informed by media campaigns on television, but also at primary care health facilities. Perhaps the increase in exposure to family planning messages on TV emboldened women to speak about the issue more forthright with their primary care provider. Not only was there more public information available about family planning, and an increase in contraceptive use, but women's knowledge of contraception also grew in practical ways to recognize modern methods. 


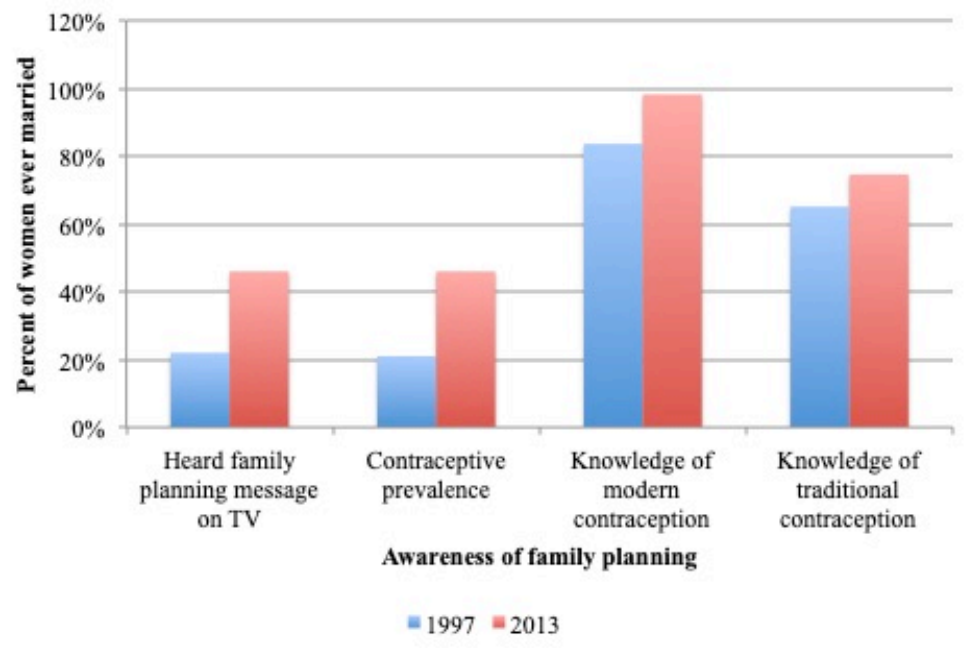

Figure 11: Awareness of family planning

An increase in the age of first marriage in both urban and rural areas demonstrates a significant cultural change and undoubted improvement of quality of life and prospects for women. By pushing back the age of first marriage, the age of first birth is a likely consequence and one that improves health outcomes for infants and their mothers. The median age of first marriage increased in both urban and rural communities. Another encouraging trend in ROY was that the median age for first birth increased slightly in both urban and rural locations. The increase in age at first birth might reflect the earlier reported result of the rising age of first marriage. 


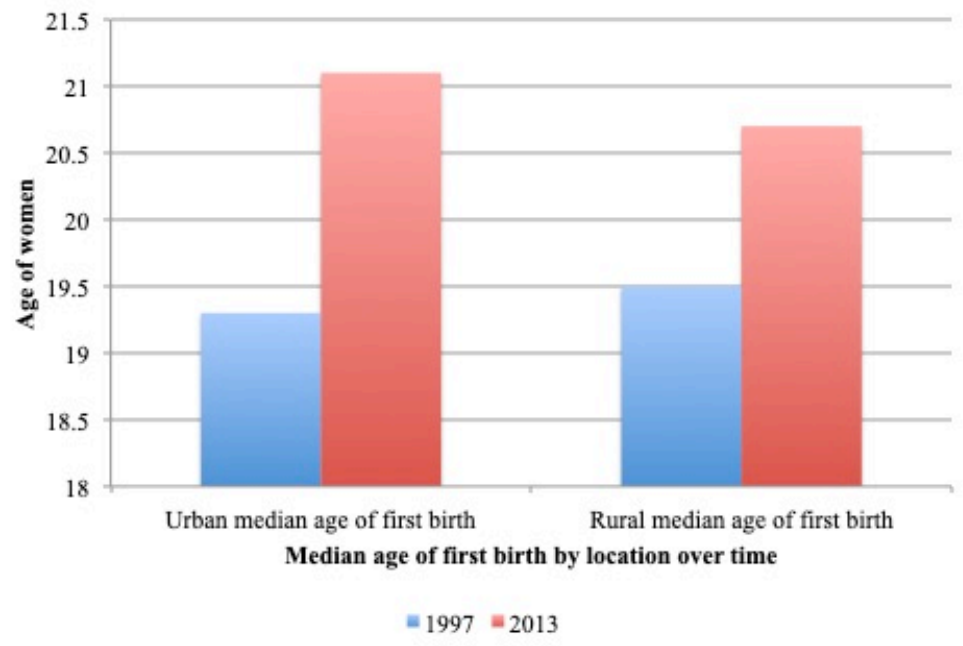

Figure 19: Median age of first birth by location over time

Birth intervals also reported improvements over time. Increased birth interval (spacing) gives women time to recover physically from childbirth. It also provides time and resources to devote to existing children in the family without having to redirect or redistribute already scarce essential resources like food or nourishment needed for breastfeeding away from a needy newborn.

In ROY the IMR by birth order was hardest on the first born with an IMR of 110.2 in 1997, then 59 IMR in 2013. This notable drop in IMR for the first-born argues that young women had more access over time to primary care and improved delivery standards. That they knew it was available in the first place, had access to it, and knew that it was preferable to have a facility birth is a tremendous achievement. If women experience a successful birth in facility with their first child and experience corresponding care for the new infant after birth they are more likely to seek that level of care with their subsequent children. As seen in the survey, all subsequent birth order babies had dramatic decreases in mortality. 
Results from the DHS (listed for both urban and rural communities) show that of women ever married the mean ideal number of children changed very little. Culturally the desire for four children remained largely unchanged. In urban areas the mean ideal number of children in 1997 was 4.2, and in 2013 it was 4. Rural areas had similar results, in 1997, 4.7 was considered the mean ideal number of children and by 2013 it was 4.5 children.

Health education, preventative action and primary care were seemingly indirect mechanisms that alleviated the proximate physiological mechanisms of infant mortality in ROY in the important phase of preconception, and the decision to delay child bearing. The choice to defer initial pregnancy, and subsequent pregnancies made strides in reducing the number of compromised deliveries and infant deaths. Reduction in IMR based on preconception choices and education and improvements in access to primary care that followed appropriate health protocols certainly were factors but intermittent war and general chaos in ROY might have also altered or forestalled decisions about marriage and family planning. 


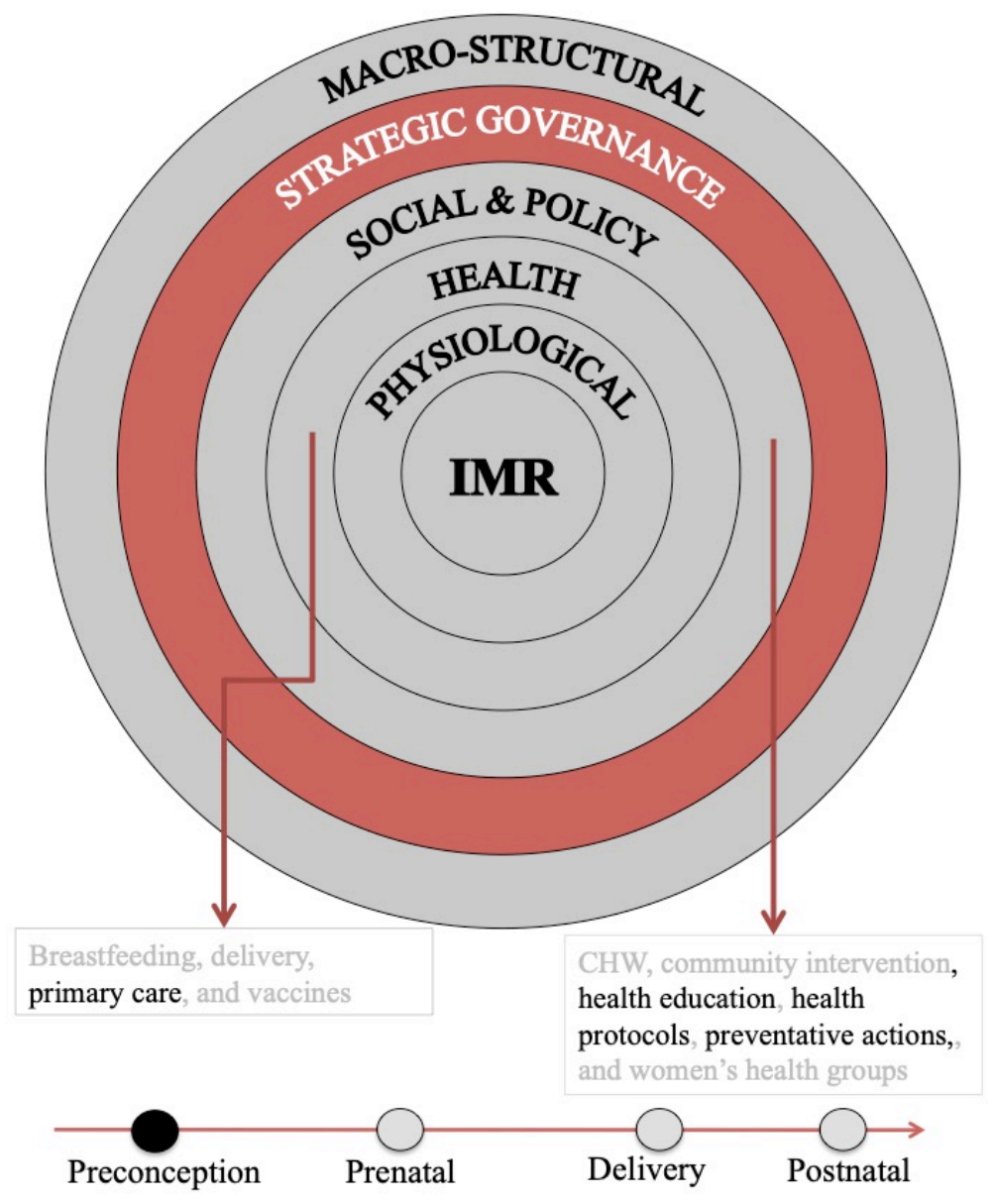

Chronology of potential care phases during a woman's life

Figure 20: Strategic governance at preconception phase (ROY)

\section{Antenatal/Prenatal}

The antenatal/prenatal phase revealed some powerful developments, especially in the increasing number of doctors attending to pregnant women and the increase in antenatal visits. However, this phase also unveils a disappointment in the level of tetanus vaccination over time (which remained stagnant) that seriously undermines the care quality provided by CHWs, primary care facilities, and doctors. This antenatal/prenatal phase reveals an opportunity for service provision quality to 
improve without the need for additional technology but more implementation science.

An encouraging trend was the source of antenatal care for pregnant women improved between the two surveys. Women received more antenatal care from doctors between the two surveys. Presumably the increase in doctor attendance to pregnant women enhanced care and quality to a degree but tetanus vaccination remained problematic. The midwife/nurse remained on the sideline in antenatal care, increasing marginally however; nearly $40 \%$ of antenatal care provision is not accounted for between the two DHS reports. ANC care from trained medical personnel almost doubled between 1997 to 2013: 34\% to 60\%. Without enough doctors to go around, it seems that CHWs and primary care facilities must have improved access and quality for women, and/or that more CHWs were able to meet with women in their homes, and that those CHWs had more skill and knowledge than previously.

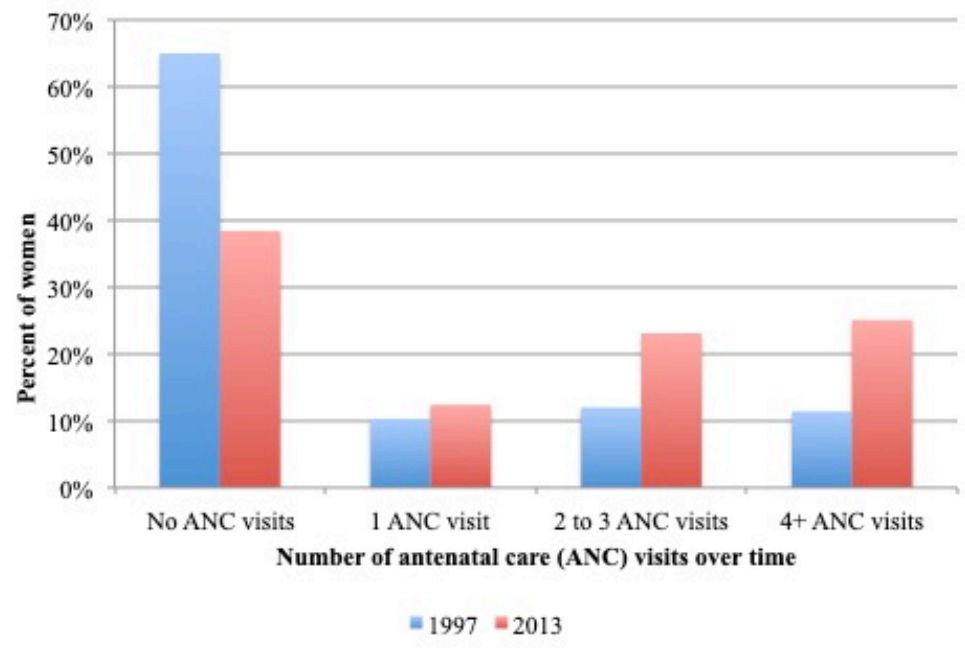

Figure 21: Number of antenatal care (ANC) visits over time 
Unfortunately, the percent of women who had tetanus toxoid vaccination during their last pregnancy of a live birth remained the same at 9\% from 1997 to 2013. This discouraging result represents uneven development in maternal and infant health care. If accurate, the tetanus vaccination rate provides a strait forward roadmap for swift change in IMR if international partnerships and funding can be harnessed. This surprisingly low tetanus vaccination rate might be the result of a input error, or indication that it was not monitored appropriately but given that it was part of both DHS reports it seems unlikely that it was not tracked. Another study provides additional information regarding vaccination in ROY. A midterm report in 2011 from the Global Immunization Vision and Strategy (GIVS) 2006-2015 of the WHO reported on vaccination progress goals of $90 \%$ coverage for each of 194 countries in regards to diphtheria, tetanus toxoid, and pertussis vaccine, and the measles vaccine ${ }^{493}$. Of particular attention were 68 countries where approximately $95 \%$ of all maternal and child deaths occur ${ }^{494}$. ROY was "on track" to achieve $90 \%$ coverage toward the third does of diphtheria, tetanus toxoid, and pertussis vaccine (DPT3) in infants under twelve months of age. Unfortunately, ROY was not on track for administering the first dose of measles vaccine, and the country was noted as having made "no progress" in that measure (Republic of Congo was

493 David W. Brown et al., "A Mid-Term Assessment of Progress towards the Immunization Coverage Goal of the Global Immunization Vision and Strategy (GIVS)," BMC Public Health 11, no. 1 (2011): 1, https://doi.org/10.1186/1471-2458-11-806.

${ }^{494}$ Brown et al., 1. 
“on track" for both DPT3 and measles ) ${ }^{495}$. The unimproved vaccination rate of measles in ROY demonstrates that the reported unchanged rate of tetanus vaccination after latest pregnancy might also be correct.

The antenatal/prenatal phase of care for women saw marked improvement (as IMRs dropped) but this phase also hinted at uneven quality health care provision as tetanus vaccination, an important indicator of infant health outcomes and an expected health protocol as part of quality maternal and infant care, was unchanged over sixteen years. Women clearly participated more in their health care through increased numbers of antenatal/prenatal visits with increasingly more trained or educated medical providers. The indirect mechanisms that alleviated the proximate physiological mechanisms of infant mortality in the antenatal/prenatal phase in chronology of care for women were improved primary care in many instances, greater engagement with CHWs, and more robust health protocols (as indicated by more antenatal visits but undermined by uneven vaccination follow through and/or reporting).

\footnotetext{
495 Brown et al., 4.
} 


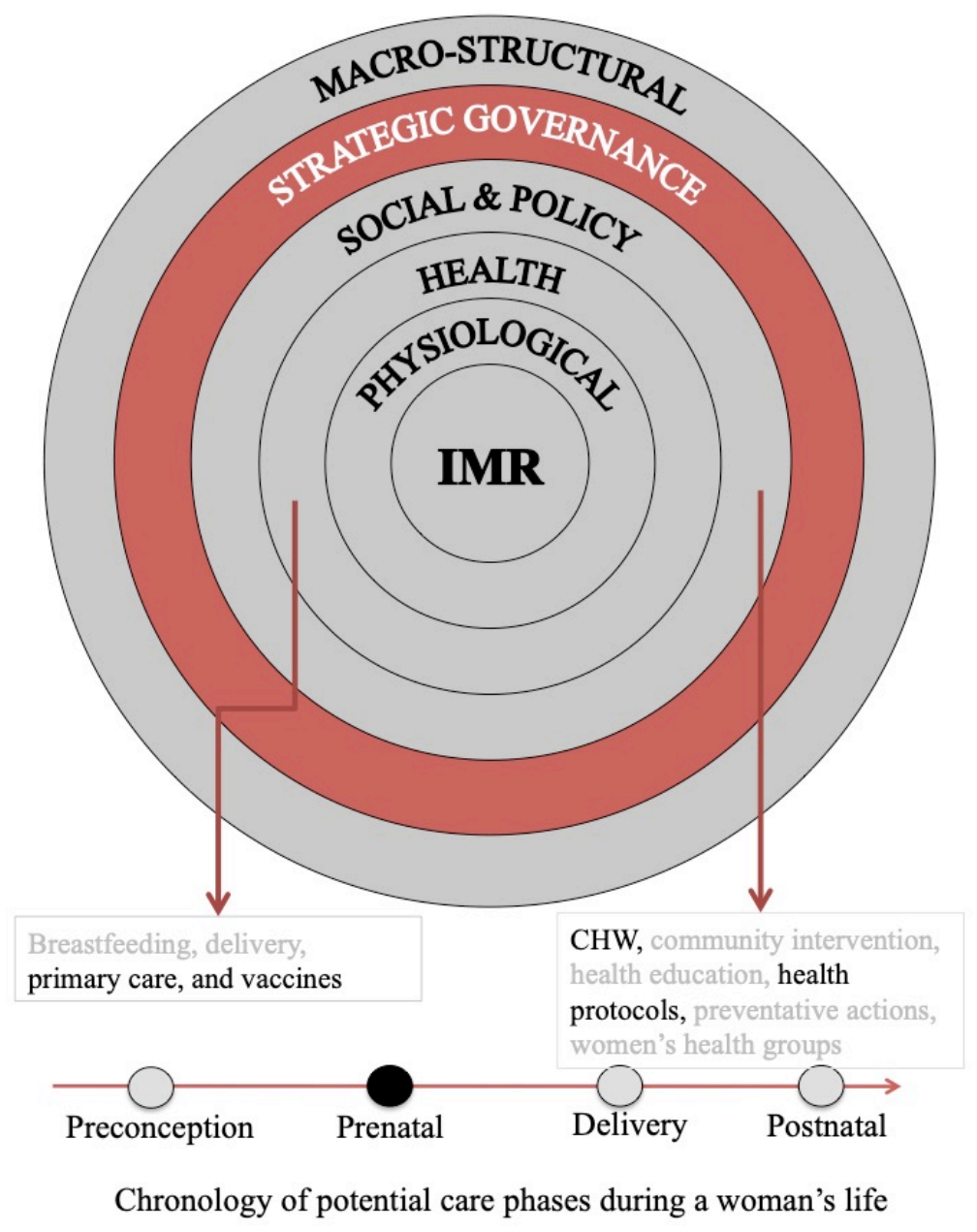

Figure 22: Strategic governance at antenatal/prenatal phase (ROY)

\section{Intrapartum/Delivery}

The limited comparable information for the intrapartum/delivery phase of the chronology of care provides a glimpse at vital improvements in ROY for IMR. The health facility as a place of delivery almost doubled but home births were still more common. Assistance at delivery by a doctor increased and nurse/midwife assisting at delivery grew. The traditional midwife assisting at delivery decreased but having no one assist at delivery also decreased. By and large having a relative assist at 
delivery was quite common and it remained the most common assistance at delivery. The indirect mechanisms alleviating the proximate physiological mechanisms of infant mortality in the delivery phase were improvements in delivery care and potentially health education. 


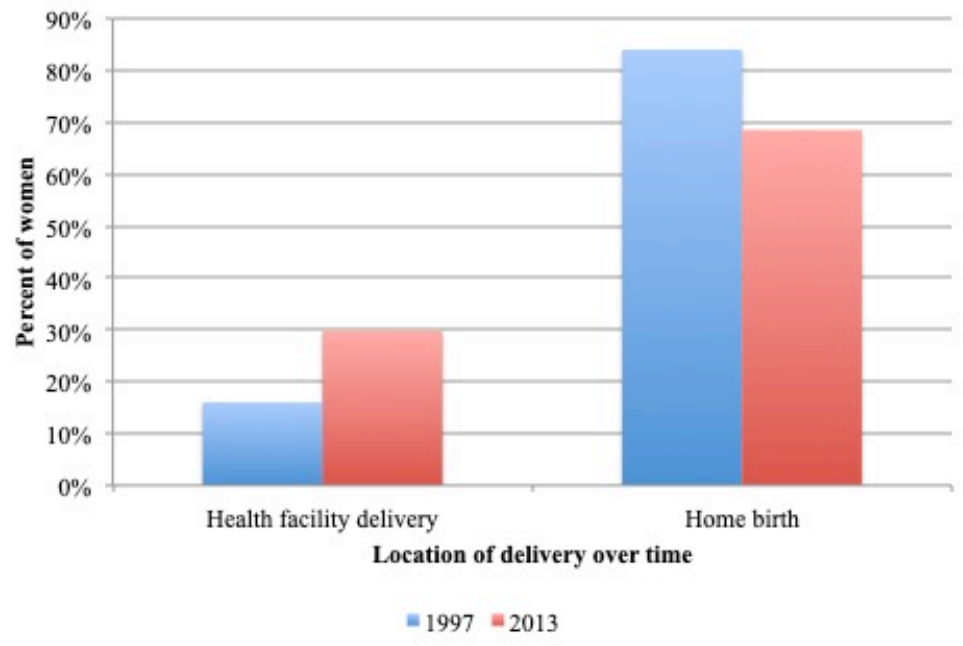

Figure 23: Location of delivery over time

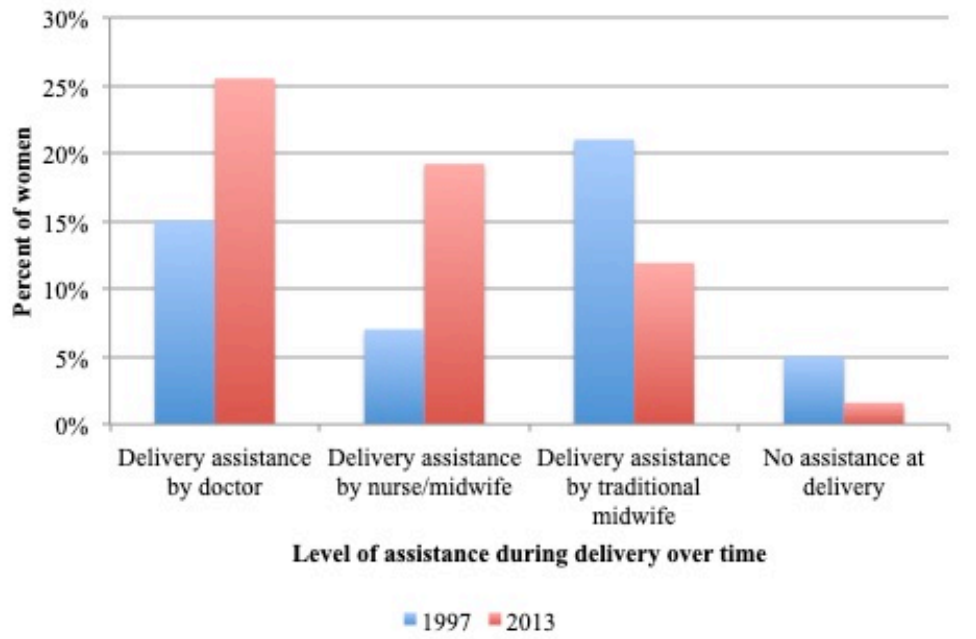

Figure 24: Level of assistance during delivery over time 


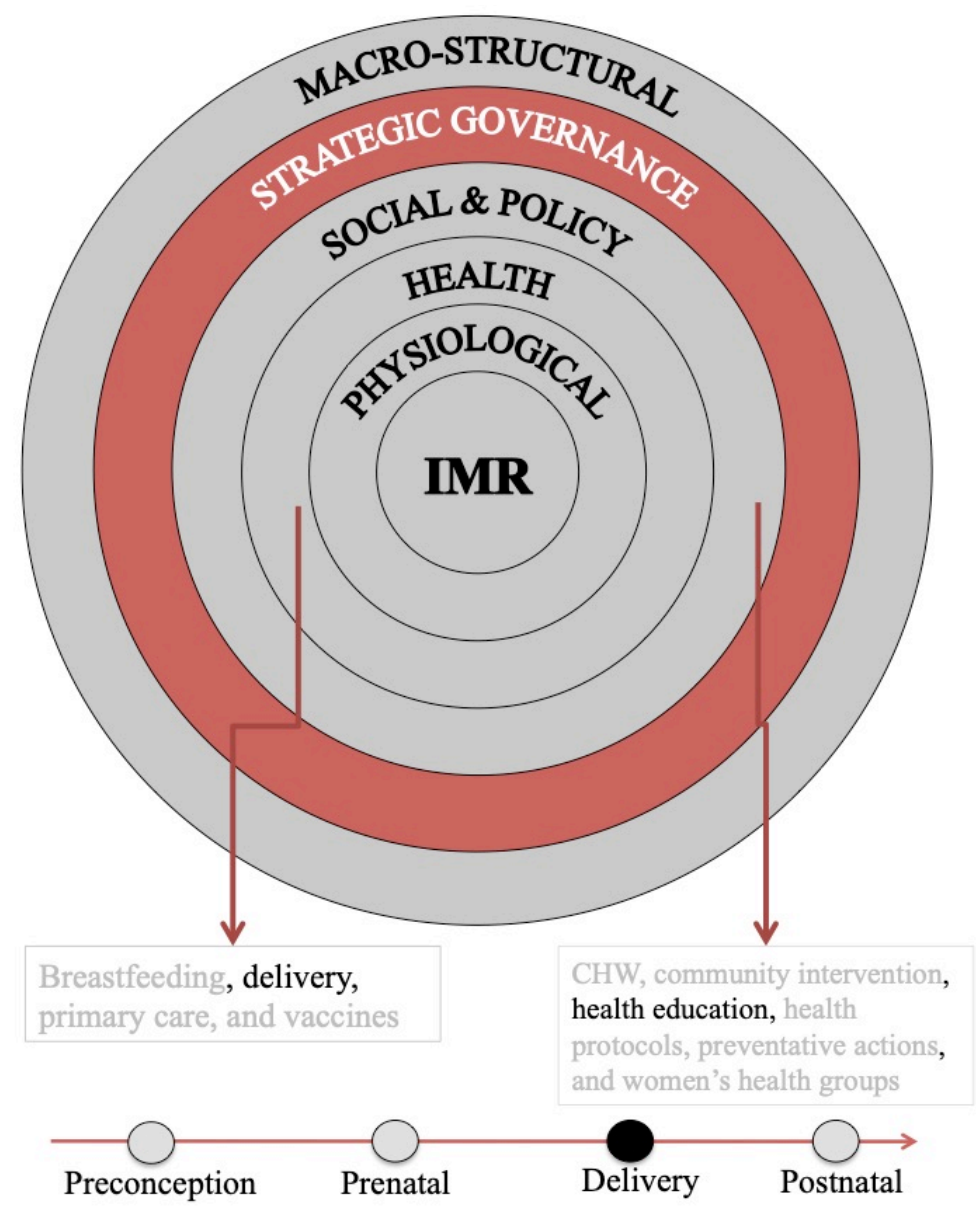

Chronology of potential care phases during a woman's life

Figure 25: Strategic governance at delivery phase (ROY)

\section{Postnatal}

There was no comparable postnatal data available from the DHS surveys for the Republic of Yemen.

e. Case study analytic narrative and analyzing the narrative

In the procedure of analytic narratives, we look for institutional drivers that have a plausible causal relationship to the outcomes of note, in this case the indirect mechanisms that alleviate the proximate physiological mechanisms of infant 
mortality. Through a review of health-related governance literature in Yemen between 1996 and 2019, four key institutional drivers were identified. Each showed a repeated pattern of causal influence over the indirect mechanisms. They are: decentralization, international partnerships, funding, and cultural change. These four institutional drivers reinforce each other and further positive outcomes in crisis conditions in ROY.

\section{The Narrative: Part I Decentralization}

One driver of change that impacted the indirect mechanisms that alleviate the proximate physiological indicators of infant mortality in ROY was decentralization. Decentralization developed out of the prior competing health systems of the former People's Democratic Republic of Yemen (PDRY) with socialist welfare state medicine, and the former Yemen Arab Republic (YAR) with both state supported and private health care systems, a combination of which continued after unification ${ }^{496}$. Ultimately the health services in Yemen were overburdened by "free access policies", relentless conflict, and under paid physicians consequently creating poor quality in the public health care system ${ }^{497}$. After unification Yemen's health spending dropped significantly impacted no doubt by the economic crisis ${ }^{498}$. By

\footnotetext{
496 Matthew Fuss, "Increasing Health Care Access in Yemen Through Community-Based Health Insurance," Indiana Journal of Global Legal Studies 23, no. 2 (2016): 802, https://doi.org/10.2979/indjglolegstu.23.2.0795.

497 Shadi S. Saleh et al., "The Path towards Universal Health Coverage in the Arab Uprising Countries Tunisia, Egypt, Libya, and Yemen," The Lancet 383, no. 9914 (2014): 371, https://doi.org/10.1016/S0140-6736(13)62339-9.

498 Jens Holst and Christian A. Gericke, "Healthcare Financing in Yemen," International Journal of Health Planning and Management 27, no. 3 (2012): 199, https://doi.org/10.1002/hpm.2107.
} 
1998, the Ministry of Public Health and Population (MoPHP) in Yemen decided to reform health care based on a decentralized model to address poor quality care and to put an emphasis on service provision and outcomes-based management ${ }^{499}$. The government recognized the necessity of decentering the service provision, planning, decision-making and financial management so that district (community) level health facilities could become more autonomous with community co-management and the ability to partner with the private sector and NGOs with goals towards innovative service provision to improve quality ${ }^{500}$. The reform program was a step towards establishing limits on the national government and a step away from being a service provider ${ }^{501}$. Opening the door to innovation and accountability, community level health facilities were able to address primary concerns and partner with organizations offering solutions and funding.

In 2000, the Ministry of Public Health created the Health Sector Reform Strategy (HRS) to improve the failing health system. The Health Sector Reform Strategy highlighted priority concerns as demonstrated by order of importance in the list below 502 :

\footnotetext{
499 Yemen Ministry of Public Health, "Leading Yemen to Better Health Safety and Well Being," Ministry of Health, August 17, 2015, http://www.moh.gov.ye/english/message.html.

500 Yemen Ministry of Public Health.

501 Yemen Ministry of Public Health.

502 Ministry of Public Health- Yemen, "Health Sector Reform in the Republic of Yemen: Strategy for Reform," October 2000,14, http://www.mophp-ye.org/docs/HSR_Strategy.pdf.
} 


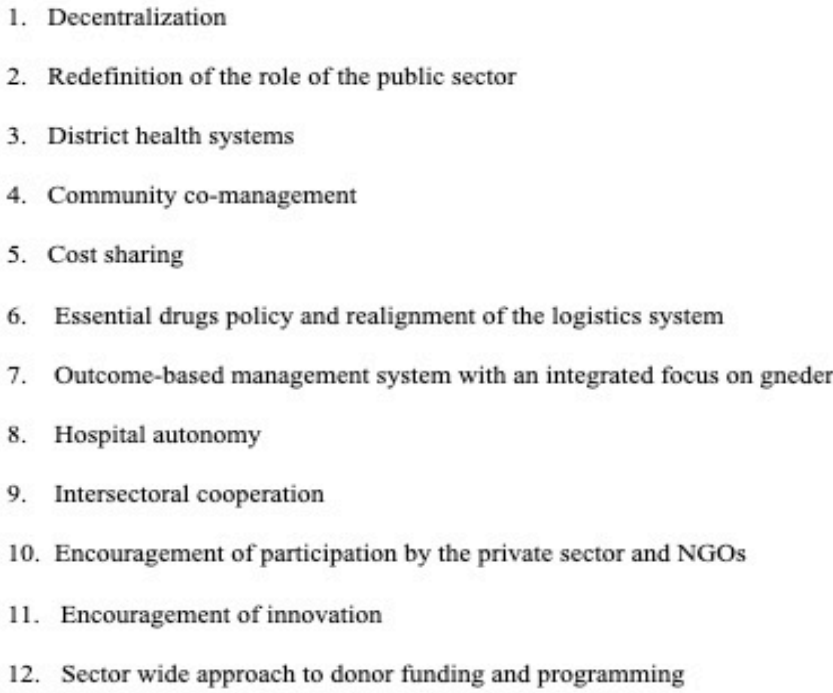

Figure 26: The 12 components of health sector reform strategy

Chief among the key components of the strategy, constructed entirely by the Ministry of Public Health, was decentralization, upon which all the other components would rely ${ }^{503}$. Decentralization was the essential reform component to encourage a failing health system by shifting responsibility for improved service provision, capacity and quality to local levels. It was hoped that local level health care provision would be more responsive to community health needs and that it would be the first line defense in health care provision rather than district, governorate and national level care facilities ${ }^{504}$. Previously, due to poor health protocols, local health facilities were frequently by passed in preference of perceived better quality health care at higher levels of health provision at the 
governorate and national level ${ }^{505}$. Local level health care refers to "district health system" which incorporates both the basic health facility level and then the community level. Decentralization of health care provision in ROY in this context means the "deconcentration" of management to the district level and in some responsibilities there was intended total "delegation" of service provision to organizations not affiliated with the Ministry of Public Health or publicly provided 506 .

Under the newly emphasized district health system, the community based health services (CBHS) were expected to reach all populations, especially those who are unable to reach facility based health staff. Community health workers that are volunteers or semi-volunteers (selected by the community and for the most part of women) are expected to be extensions of health facility based staff, and serve as "trained traditional birth attendants, community educators or contact mothers for MCH/FP" reaching the unreached ${ }^{507}$. The Ministry of Health also identified diseases as priorities for district level health care. The following diseases were selected because of the impact on public health and relatively uncomplicated ease of intervention to prevent or cure the diseases over shorter timeframes ${ }^{508}$ :

505 Ministry of Public Health- Yemen, 18. 506 Ministry of Public Health- Yemen, 15. 507 Ministry of Public Health- Yemen, 19. 508 Ministry of Public Health- Yemen, 44. 

1. Diarrhea
2. Malnutrition
3. Complications of pregnancy
4. Delivery and puerperium
5. Acute respiratory infections
6. Malaria
7. Schistosomiasis
8. Tuberculosis
9. Accidents
10. Hepatitis
11. AIDS
12. Leprosy

Figure 27: Priority district level health care diseases, Ministry of Public Health 2000 Yemen

Analyzing the Narrative: Decentralization

Decentralization in ROY influences the indirect social and policy mechanisms (like community health workers and women's health groups) that alleviate the proximate physiological mechanisms of infant mortality. The proximate health mechanisms that alleviate the proximate mechanisms of infant mortality are delivery, primary care and vaccination.

The decision to decentralize health care provision came from the realization that the national government did not have the capacity or the budget to provide comprehensive health care, nor perhaps even the intent to provide health care services. Limiting the role of the public sector was necessary in order to move forward based upon the reality in ROY, i.e., capacity of national government, number 
of current workers, local facilities, and geographic needs ${ }^{509}$. Through

decentralization, the entire chronology of potential care phases during a woman's life is impacted and that had the potential to improve IMR.

A study in Spain demonstrated that decentralization of health care, per say, did not improve infant mortality, but when health care decentralization was coupled with political and fiscal responsibility (as it was in Yemen) for health outcomes in regions, there was a $17 \%$ drop in IMR, and a $19 \%$ drop in neonatal mortality 510 . The study also points out that countries that have democratic institutions have longer life expectancy and lower IMR, in one study about $17 \%$ fewer infant deaths per 1,000 than autocratic countries ${ }^{511}$. It is therefore reasonable to expect that given the restrained capacity of the national level and the devolution of health care responsibility to local levels with a focus on primary care and female community health workers that the IMR would improve because communities were able to chose for themselves the best course of action for their circumstances. In fact, a decentralized center of administration in the case of Yemen where tribalism is the only bureaucratic system many in the country have experienced might not have appeared much different ${ }^{512}$. Tribalism in Yemen has been considered a "shadow

\footnotetext{
509 Ministry of Public Health- Yemen, 17.

510 Dolores Jiménez-Rubio and Pilar García-Gómez, "Decentralization of Health Care Systems and Health Outcomes: Evidence from a Natural Experiment,” Social Science \& Medicine 188 (2017): 73, https://doi.org/10.1016/j.socscimed.2017.06.041.

511 Timothy Besley and Masayuki Kudamatsu, "Health and Democracy," in The American Economic Review., vol. 96, 2006, 315, https://doi.org/10.1257/000282806777212053.

512 Dingli, "Is the Failed State Thesis Analytically Useful?," 97.
} 
government"513 as it has provided "core functions" with external financial assistance ${ }^{514}$. The shadow government of tribalism in the local Yemeni context can mean local groups attend to community needs. At the local level some service provision like schools, water and roads have predominantly been delivered in Yemen through Local Development Associations (LDAs), "local institutions linked through an electoral process to the Confederation of Yemeni Development Associations (CYDA)"515. Even as Saleh consolidated more power after the civil war through constitutional changes there were equally focused regional attempts to weaken his control through the expansion of civil society with "self-help groups and local communities fending for themselves"516. Additionally, the limited (or nonexistent) road networks in rural areas of Yemen impacted access to health care ${ }^{517}$ so having an emphasis on locally available and locally sensitive health care responsive to specific community needs undoubtedly improved health outcomes. Health service providers were able to implement timely life saving procedures in the critical stage of delivery and perinatal care. As we have seen, several conditions drive health outcomes of newborns and they are primarily related to the health of the mother, her nutritional status, birthing conditions, and the quality of neonatal

\footnotetext{
513 Dingli, 97.

514 Dingli, 98.

515 Carapico, Civil Society in Yemen the Political Economy of Activism in Modern Arabia, 108. 516 Blumi, Destroying Yemen, 160.

517 Abdullah Al-Taiar et al., "Physical Accessibility and Utilization of Health Services in Yemen," International Journal of Health Geographics 9, no. 1 (2010): 38-38, https://doi.org/10.1186/1476072X-9-38.
} 
care $^{518}$. Delivery is the culmination of care the mother has received, and is often indicative of the care the newborn will receive. Previous centralized decisionmaking often focused on infrastructure, particularly in urban areas, rather than on quality care (especially perinatal care), or following established health protocols, training for medical professionals and access to care (which is a concern to poor and rural families $)^{519}$. The government recognized the necessity of decentralizing care to the health facility level and in doing so supported more efficient use of resources.

The Narrative: Part II International Partnerships

Another driver of change that impacted the indirect mechanisms that alleviated the proximate mechanisms of infant mortality in ROY was international partnerships. International partnerships provided expertise and guidance on how to implement desired and necessary health care reforms.

In 2004, the Ministry of Health held talks with stakeholders to address quality concerns in hospitals ${ }^{520}$ indicating interest in improving health outcomes. The Government of Yemen and its development partners (UNICEF, GTZ, and the World Bank) undertook a study to address shortfalls in emergency obstetric and neonatal care (EmONC). A needs assessment study conducted in 2009 showed a continued

${ }^{518}$ Qaiser Khan and Susan Chase, Yemen and the Millennium Development Goals (World Bank, 2003), 13.

519 Khan and Chase, 14-15.

520 John Øvretveit and Abdul Al Serouri, "Hospital Quality Management System in a Low Income Arabic Country: An Evaluation," International Journal of Health Care Quality Assurance 19, no. 6 (2006): 518, https://doi.org/10.1108/09526860610686999. 
shortage of health professionals, particularly in rural areas ${ }^{521}$. Partnerships provided funding and technical assistance to evaluate ongoing needs in Yemen. Findings pointed to poor conditions at the point of delivery. The need in rural Yemen far exceeds the government's ability to provide even basic services and/or infrastructure ${ }^{522}$. For instance, in the urban governorate of Aden, there were 54 obstetricians for 494 expectant mothers per year, but in the rural governorate of Shabwa, 1 obstetrician for 21,087 expectant mothers per year. Or, again in Aden there were 72 pediatricians and in Shabwa there were 3: the recommended ratio is 1 obstetrician per 350 pregnant women ${ }^{523}$. Later in 2011, Yemen developed a National Health Strategy to focus on quality in preventive, curative and rehabilitative services ${ }^{524}$. Through leadership and partnership, the World Health Organization was able to support the MoPHP improve quality of education and services provided by health professionals in medical schools, health institutes, a nursing programme, the Yemeni Board of Medical Specialization, and the Human Resources Department in the Ministry of Public Health and Population ${ }^{525}$. Changing the landscape of quality health services

\footnotetext{
${ }^{521}$ Abdul Wahed Al Serouri et al., "Findings from a Needs Assessment of Public Sector Emergency Obstetric and Neonatal Care in Four Governorates in Yemen: A Human Resources Crisis," 
improved the patient experience and encouraged patient's to trust the advice and recommendations of health professionals.

In 2012, the Maternal and Child Health Integrated Program (MCHIP) in Yemen began, "with field support funding" from the U.S. Agency for International Development (USAID). A “Quick Start (QS) technical assistance program” was created to assist MoPHP support reproductive, maternal, newborn, and child health and nutrition $(\mathrm{RMNCH} / \mathrm{N})$ at both the national and governorate level in selected areas $^{526}$. During the project many technical aspects of quality care were addressed: in conjunction with the United Nations Children's Fund (UNICEF) evaluations of "community-based maternal and newborn care programs (CBMNC)" were conducted, and studies with the Higher Institute of Health Sciences (HIHS) to support a new "community-based education program and improve access to highquality midwifery services" were implemented ${ }^{527}$. The Quick Start program also emphasized building partnerships (domestic and international) to maximize the capacity of established programs ${ }^{528}$. MCHIP partnered with the United Kingdom Department of International Development and the European Union on a nutrition program, the Expanded Program on Immunization initiative, the Japanese International Cooperation Agency and WHO on a community project, with Deutsche Gellschaft fur International Zusammenarbeit (GIZ) on a Quality Improvement

\footnotetext{
526 USAID, “MCHIP End of Project Report," 2014, 244.

527 USAID, 244.

528 USAID, 246.
} 
Program, with the United Nations Population Fund for community midwife education and improving midwifery in general, as well as with the Faculty of Medicine and the Faculty of Applied Sciences at University of Dhamar, the Yamaan Foundation, the National Safe Motherhood Alliance, the Yemen Midwives Association, and Yemen Family Care Association ${ }^{529}$. The focus on improving the continuum of care for women and infants through improved health protocols throughout antenatal care was an important aspect of improving the IMR.

\section{Analyzing the Narrative: International Partnerships}

International partnerships in ROY seem to influence the indirect social and policy mechanisms that alleviate the proximate physiological mechanisms of infant mortality that impact community health workers, health education, community interventions, women's health group, preventative actions and health protocols. Breastfeeding, delivery, primary care, and vaccines impacted the indirect health mechanisms. International partnerships were necessary to facilitate improved quality health delivery at during every phase of the continuum of care for women. One twelve year hospital based study in Sana'a's Al-Sabeen Hospital revealed that poor delivery care has been overcome, in some instances, through an inter-hospital partnership of sharing specialist doctors. A new "standing practice", according to the study (2005), is that specially trained pediatricians attend the delivery of at-risk

529 USAID, 246. 
pregnancies to address intensive neonatal care needs ${ }^{530}$. The hospital study demonstrated a $40.5 \%$ decrease in the number of early neonatal deaths (ENND) between over the study years. The partnership of hospital networks allowed for the sharing of specialist resources to enhance quality of care at time of delivery. The additional education of the pediatricians in specific "intensive neonatal care" abroad and locally was an intervention that benefited local hospitals that collaborated in local partnership to ensure maximum coverage of a scarce health expertise. Partnership also improved the maternal mortality rate, which in turn improved the IMR. 82\% of maternal deaths occur intrapartum, or at delivery, so improving obstetric care is essential ${ }^{531}$. A study in 2009 highlighted that the maternal mortality rate in Yemen was 365 per 100,000 live births ${ }^{532}$, and in 2013 it was 148 per 100,000 live births ${ }^{533}$. The study shows that the Government of Yemen in conjunction with multiple international organizations (United Nations Children's Fund, United Nations Population Fund, World Bank, and the German Agency for Technical Cooperation) were focused on reducing maternal mortality; however, their needs assessment in 2009 revealed that there were gaps in access to primary care and concerns of quality to overcome. Based on the findings, The World Bank HRSP introduced funding to support emergency obstetric care (EmOC) in three

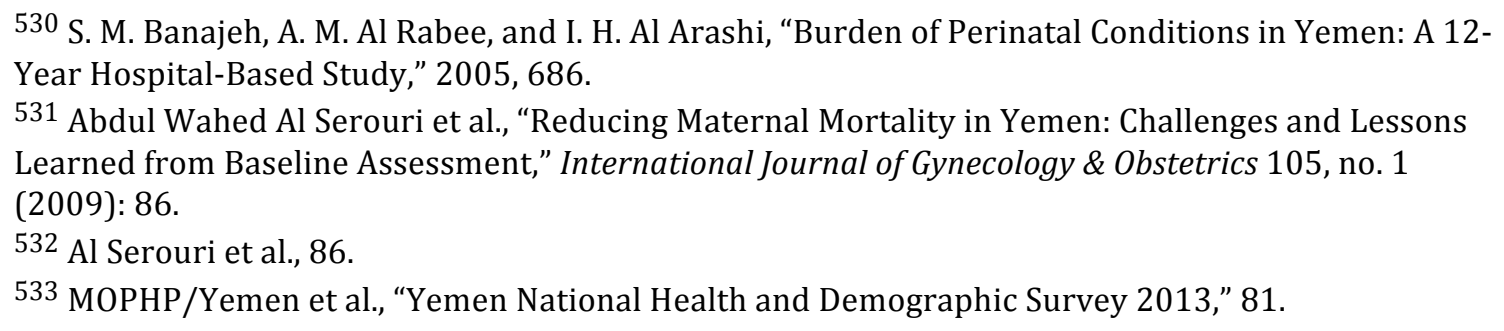


district hospitals, and twelve health centers that "were chosen for their remoteness, neglect, and generally poor quality services" 534 . The decentralized focus on behalf of the World Bank on quality care for the remote and neglected undoubtedly brought much needed support and funding for struggling communities. Efforts to improve were focused on organizing a system to monitor maternal mortality, EmOC training for doctors and midwives, disseminating clinical guidelines and essential protocols, and regular supervision to verify compliance with established protocols 535 . Improving the quality of health provision and education for health professionals through partnership with international organizations and appropriate funding improved health outcomes for mothers and infants.

\section{The Narrative: Part III Funding}

Funding was another important driver of change that impacted the indirect mechanisms to alleviate the proximate physiological mechanisms of infant mortality in ROY. Funding is frequently connected to international partnerships. For instance, an international partnership pilot program that addressed the shortage of primary care, prenatal care, and delivery care in rural Yemen was the Extending Service Delivery (ESD) Program. This program supported the Basic Health Services (BHS) Project to aid midwives in setting up their own private practices in rural Yemen

\footnotetext{
534 Al Serouri et al., "Reducing Maternal Mortality in Yemen," 91.
}

535 Al Serouri et al., 91. 
communities ${ }^{536}$. USAID funded BHS so that twelve midwives could serve nine BHS governorates where health services and facilities where unavailable. Where about $77 \%$ of the population was rural and only $25 \%$ of the rural population had access to health services, twelve new private midwives serving the remote and neglected areas of Yemen was a step in the right direction although it must be recognized as only a first step ${ }^{537}$. In 2004 a group of midwives in Yemen established a nonprofit midwives association (YMA) modeled after professional organizations in other countries- the YMA received funding and specialist support from the European Union, GTZ, International Confederation of Midwives (ICM), PHRplus Project (USAID-funded), The United Nations Population Fund (UNFPA), USAID/Yemen and the WHO538. BHS (funded by USAID) worked closely with the YMA and the twelve private midwives to establish another 56 private midwives that worked in under served communities ${ }^{539}$. Another program, supported by USAID through the ESD Program, in partnership with MoPHP was to scale up best practices in postpartum care in an urban community at the Al Saba'een Hospital. The successful best practices implemented at Al Saba'een Hospital led to the implementation of: Kangaroo Mother Care (KMC), immediate and exclusive breastfeeding, prevention of neonatal infection,

\footnotetext{
536 J Kesselman, "Private Midwives Serve the Hard-to-Reach: A Promising Practice Model," IntraHealth, 2010, https://www.intrahealth.org/sites/ihweb/files/attachmentfiles/ESD_Legacy_Yemen_Private_Midwives_6_24_10.pdf.

537 Kesselman.

538 Kesselman, 3.

539 Kesselman, 3.
} 
postpartum family planning (that included men for the first time), neonatal immunization (BCG and Polio), active management of third stage of labor, and neonatal resuscitation- all essential elements in the prevention of infant mortality ${ }^{540}$. The $\$ 50,000$ grant from ESD in partnership with MoPHP launched best practices that impacted postpartum women and newborns, as well as health professionals ${ }^{541}$. The scale up of best practices expanded to over fifteen hospitals with plans on behalf of BHS and MoPHP to "scale up to 138 more rural hospitals, primary health care and maternity centers" ${ }^{\prime \prime 2}$. Initial results in six of the fifteen hospitals demonstrated that immediate breastfeeding of newborns went from $0 \%$ in October 2009 to $78 \%$ March 2010, and family planning after delivery went from $0 \%$ to $73.5 \%$ in the same timeframe ${ }^{543}$.

\section{Analyzing the Narrative: Funding}

Funding in ROY seems to have influenced the indirect health mechanisms (i.e., breastfeeding, delivery, primary care and vaccines) that ultimately impact infant mortality. Indirect social and policy mechanisms likely to be impacted by funding are community health workers, health education, community intervention, women's health groups, preventative action and health protocols. Dramatic results were possible with funding, as there was a dearth of quality care (often from lack of

\footnotetext{
${ }^{540}$ Salwa Bitar, "Accelerating the Spread of Best Practices in Postpartum Care: Scaling-Up Best Practices in Yemen," IntraHealth, 2010, 1, https://www.intrahealth.org/resources/acceleratingspread-best-practices-postpartum-care-scaling-best-practices-yemen.

541 Bitar, 3.

542 Bitar, 2.

${ }^{543}$ Bitar, 3.
} 
supplies, medical personnel, or facilities). The funding frequently came through international partnerships in support of specified local projects. In addition to funding, there was probably the expectation of measureable results to show for the investment. Although not specifically tied performance-based funding as in ROC, international partners probably had an interest in the outcomes financial donations. Frequently, funding "buys" participation, if not management, of particular programs. The increased interaction with funding partners encourages new norms and standard operating procedures that ultimately contribute to a change in culture.

\section{The Narrative: Part IV Culture}

The culture of ROY was another driver of change that impacted the indirect mechanisms of infant mortality. Improving odds of infant survival came partly from changes in the culture of family planning. DHS data reveal that family planning messages heard by women on television have increased almost twofold, contraceptive use was up and knowledge of family planning has increased to almost at $100 \%$. The longitudinal data from the DHS show an increase in knowledge among ever married women. The trend of increased knowledge of family planning prepared women to make choices that were more beneficial especially given changes in marriage laws.

In 1999, Yemen's Personal Status Law, article 15, which established a minimum age of 15 for boys and girls for marriage, was eliminated for religious reasons. In 2006, a survey highlighted that $14 \%$ of Yemeni girls are married before age 15 , and slightly 
more than half are married before they turn $18^{544}$. Another study from Sana'a University revealed girls are sometimes married by age $8^{545}$. Yemeni Noble Laureate Tawakkol Karman (2011) brought child marriage to the forefront through her activism against gender abuses ${ }^{546}$.

In addition to activism there are numerous international conventions that Yemen is a party to that call for the eradication of child marriage such as the "Convention on the Rights of Child, the Convention on the Elimination of all Forms of Discrimination against Women, the Convention on Consent to Marriage, Minimum Age for Marriage and Registration of Marriage, the International Covenant on Civil and Political Rights, and the International Covenant on Economic, Social and Cultural Rights"547.

\section{Analyzing the Narrative: Culture}

Culture in ROY influences the indirect social and policy mechanisms (i.e., community health workers, health education, community intervention, women's health groups, and preventative actions) and indirect health mechanism (like primary care and delivery) that alleviate the proximate mechanisms of infant mortality. Educating women to wait at least 24 months between deliveries is healthy for both mother and

\footnotetext{
544 Nadya Khalife, "How Come You Allow Little Girls to Get Married? Child Marriage in Yemen.," 2011, 2.

545 Khalife, 2.

546 Khalife, 2.

547 Khalife, 3.
} 
baby according to the World Health Organization ${ }^{548}$. Having short interpregnancy intervals (IPI) is associated with babies being born premature, and prematurity is the "leading cause of perinatal mortality"549. The greater the education level of the mother, the more likely she is to have more lengthy IPIs. For instance, a mother with more than 13 years education level in Yemen is not likely to have a baby with fewer than 12 months of separation between delivery and conception, whereas a woman with no education has a $60 \%$ chance of having an IPI of less than 12 months 550 . Adolescent pregnancy also increases the risk of having premature babies ${ }^{551}$. Knowledge of family planning can improve health outcomes by preventing adolescent births and lengthening the IPI.

Family planning is not always utilized because of several factors: low confidence in health system, cost, and "cultural barriers"552. A study in four districts in Sana'a, Yemen found "the major reason for not using family planning was husband's refusal"553. Cultural or social norms impact women's access to preventative family planning but cultural norms are slowly changing even in the context of conservative

\footnotetext{
548 Centre National de la Statistique et des Études Économiques- CNSEE/Congo and O. R. C. Macro, “Congo Enquête Démographique et de Santé 2005," July 1,2006, https://dhsprogram.com/publications/publication-FR182-DHS-Final-Reports.cfm.

${ }^{549}$ A. Bukair, A. W. Al-Saqladi, and A. Al-Sadeeq, "Interpregnancy Interval and the Risk of Preterm Birth: A Case-Control Study of Infants Born at Al-Sadaqa General Teaching Hospital, Aden, Yemen," International Journal of Reproduction, Contraception, Obstetrics and Gynecology 5, no. 4 (2016): 1181. 550 Bukair, Al-Saqladi, and Al-Sadeeq, 1183.

551 Bukair, Al-Saqladi, and Al-Sadeeq, 1185.

552 H. O. Basaleem, "Women's Reproductive Health Seeking Behavior in Four Districts in Sana'a, Yemen: Quantitative and Qualitative Analysis," Journal of Community Medicine and Health Education 2, no. 153 (2012): 1 .

553 Basaleem, 1.
} 
Yemen ${ }^{554}$. Culturally, condoms are gaining in use as part of family planning even as debates about their suitability continue in light of HIV/AIDS concerns as a method that condones "risk behavior" 555 . In addition, preventing child marriage disrupts adolescent pregnancy. Child brides are prevalent in Yemen. Girls who are married young have little to no knowledge of family planning, and they have little agency "against older husbands to negotiate family planning"556. Challenging the culture of child brides is slowly making progress but it has experienced setbacks.

Discouraging the cultural acceptability of child marriage will continue to minimize the IMR because of fewer pregnancies by young girls, which decreases babies born of compromised circumstances (likelihood of premature birth due to age of mother). Decreasing fertility in Yemen results in babies that are healthier, and healthier babies decrease the risk of neonatal mortality ${ }^{557}$. Cultural persuasion is steady as family planning and condoms are becoming more acceptable, child marriage unacceptable, and the rights of women recognized. Partnerships with international organizations that support the cultural shift in Yemen are key because the national government struggles against historical intolerance of women's rights that are

\footnotetext{
554 R. Busulwa et al., "Perceptions of the Condom as a Method of HIV Prevention in Yemen," 2006, S75.

555 Busulwa et al., S76.

556 Khalife, "How Come You Allow Little Girls to Get Married?," 2.

557 Khan and Chase, Yemen and the Millennium Development Goals, 13.
} 
considered "contrary to Sharia, Islamic law"558. Absent national policy against the violence inflicted on young girls, partnerships with international organizations can shape norms around more universal values. The change in culture shapes women's access to family planning education, primary care providers, and prevents fewer compromised pregnancies, which ultimately can improve infant mortality.

Another study shows that Yemeni women avoid going to a health facility for delivery because they feel they lack "authority" over the experience of childbirth. While it must be underscored that lack of health facilities and the dearth of medical professionals drive persistent "high home delivery rates", the perceived quality of care provided, represented by the frighteningly high maternal mortality rates in health facilities, are some of the "strongest predictors of utilization of services and delay in seeking care"559. Quality attended care at delivery is recommended for women giving birth but if the quality is such that it causes distrust and fear of negative health outcomes then women will continue to reject facility based births. Improved quality could draw more pregnant women to health facilities for delivery but only if there is a change in the culture and perception of how women are treated while not in their home ${ }^{560}$.

558 Khalife, "How Come You Allow Little Girls to Get Married?," 3.

${ }^{559}$ Annica Kempe et al., "Yemeni Women's Perceptions of Own Authority during Childbirth: What Does It Have to Do with Achieving the Millennium Development Goals?," Midwifery 29, no. 10 (2013): 1182.

560 Central Statistical Organization/Yemen and Macro International, "Yemen Demographic and Maternal and Child Health Survey 1997," 1998, http://dhsprogram.com/publications/publicationFR94-DHS-Final-Reports.cfm. 


\section{f. Initial statement of "strategic governance" findings}

This chapter identified four likely institutional drivers of IMR decline in ROY. The analysis suggests that decentralization, international partnerships, funding and cultural change contributed to conditions under which the indirect health, and social and policy mechanisms that tend to alleviate the proximate physiological mechanism of infant mortality arose. Strategic governance in the context of ROY might be a combination of these four institutional drivers that constitute an unintentional roadmap for IMR improvement in crisis conditions. For instance, the best evidence of this was the decision to decentralize health care that also further facilitated the other three drivers of IMR. Decentralization of service provision from the national level to the local level created a window of opportunity for international partnerships to intervene with technical expertise and with that partnership opportunity often came financial support, and cultural change over time. The decision by the Ministry of Public Health and Population to decentralize health care provision put responsibility and accountability in the hands of decision makers familiar with existing capacity in the various geographical contexts. While there was regional fighting, the absence of national level chaos and detachment from health care provision allowed choices to be made at the local level that directly impacted the indirect health, and social and policy mechanisms that alleviated the proximate physiological mechanisms of infant mortality.

The institutional drivers of decentralization, international partnerships, funding, and cultural change operate in an interactive and complex manner, generally 
reinforcing each other. As was the case in ROC, some of the institutional drivers in ROY appear to be endogenous, directly established by the government like the resolution to decenter health care service provision, or laws that impact cultural change like child marriage. Other institutional drivers (like international partnerships and funding) suggest an exogenous intervention that improves IMR. Decentralization of health care removed the expectation that national level government had capacity to address the well being of the population. With the focus on local level service provision and partnership opportunities with community groups, private sector, and NGOs, health care was able to consolidate capabilities to address the failing health sector in ways that were relevant to the regional population. Removing the dysfunctional macro-structural institution as service provider resulted in local service providers looking for additional capacity in the form of partnerships. International partnerships provided additional technical and advisory capacity to local level health care programs. Through collaboration and focused efforts, international partnerships were able to provide pathways to improved quality throughout all phases of the chronology of care for women. In the case of funding, the supplemental assets proved invaluable to support training, best practices implementation and shortages in rural areas. The funding from international partners probably brought heightened involvement to see measureable outcomes for the investment but the literature does not speak to this as it did in ROC. 
Cultural change was impacted by endogenous events and over time by exogenous forces of international partnerships and funding. Donor countries and organizations likely required certain outcomes that shaped behavior and ultimately culture. 


\section{Chapter 6: Research Findings on Strategic Governance}

Using analytic narrative case studies to explore the significant reduction in IMR in developing countries, this paper finds that micro-strategic governance can facilitate improved infant mortality in crisis conditions. Strategic governance, in ROC and ROY, appears to be linked to institutional drivers of change like decentralization, international partnerships, funding, performance-based funding and cultural change. Strategic governance might be partly what mitigates the macro-structural pessimism that threatens IMR. In both ROC and ROY many of the components of strategic governance were primarily the same but this might not be the case in other countries. Strategic governance appears to be more opportunistic than intentional, taking advantage of situations despite crisis. These findings align with the appeal made in 1971 by the development economist and Nobel laureate Albert Hirchsman, then working at Harvard University, in his widely read book A Bias for Hope: Essays on Development and Latin America. In that book, Hirschman wrote of "possibilism" as a way to reject the broad consensus thinking that had concluded that either "social and political transformation" or institutions of investment and aid were the only paradigms to development ${ }^{561}$. Through possibilism he rejects the "straightjacketing constructs" of formulaic paradigms thinking. Instead, Hirschman proposed a broader path to "increase the number of ways in which the occurrence

\footnotetext{
${ }^{561}$ Hirschman, A Bias for Hope: Essays on Development and Latin America, 29.
} 
of change can be visualized"562. Indeed, Hirschman points out that crisis conditions can provide critical energy (or even "blessings in disguise") to upend development roadblocks if one know to looks for them, and they are not in the usual places. That appeal resounded widely in the development community at the time because of poor results of decolonization. Jeremy Adelman, of Princeton University, noted that Hirschman proposed through possibilism "a re-encounter with a social science that finds hope in disappointment, solutions in tension, and liberty in uncertainty"563. This heterodox proposal wanted to banish the "gloomy visions" as Hirschman called them, of predestined thinking to "reset the imagination, to take the intellectual to consider combinations that might take anomalous, deviant, or inverted sequences and make them a potential course"564. Emre Özçelik, of Middle East Technical University, notes Hirschman put an emphasis on the "'small ideas' and lesser thoughts"' as a means to "generate 'great insights of practical value'"'565, rather than the grand or preconceived. Phillip H. Lepenies, of University of Berlin, concludes that possibilism is "solution-oriented" as Hirschman focuses on "endogenous solutions"566 precisely by avoiding sweeping panaceas promoting revolution, or exogenous intrusion through instruction and aid from

\footnotetext{
562 Hirschman, 29.

563 Jeremy Adelman, Worldly Philosopher The Odyssey of Albert O. Hirschman (Princeton, N.J.: Princeton University Press, 2013), 15.

564 Adelman, 4.

565 Emre Özçelik, “Albert O. Hirschman: A 'Beamish'Social Scientist for Our Grandchildren,” Development and Change 45, no. 5 (2014): 1115.

566 Philipp H. Lepenies, "Possibilism: An Approach to Problem-Solving Derived from the Life and Work of Albert O. Hirschman," Development and Change 39, no. 3 (2008): 456, https://doi.org/10.1111/j.1467-7660.2008.00487.x.
} 
well-intentioned institutions. Instead, he wants to look for the mechanisms that induce endogenous change "through which a suboptimal situation can be overcome or at least improved from within ${ }^{567}$. We saw two endogenous institutional drivers of change in decentralization and cultural change in the ROC and ROY case studies that were surprisingly integral to IMR improvement based on the analytic narratives.

In ROC and ROY, the chronology of care for women presented many large and small opportunities for indirect mechanisms to alleviate the proximate physiological mechanisms of infant mortality. Change was not necessarily the result of larger paradigm shifts in the case countries; on the contrary chaos persisted in many instances and yet incremental, frequently agency-centered innovation happened nonetheless. Bad structural conditions did not prevent progress. Hirschman might describe this agency-centered vision of strategic governance as the "possible" superseding the "probable" given macro structural pessimism as reason for optimism $^{568}$. Looking for advantage, even when none seems possible, and finding it rather than relying solely only what is likely or probable in given dire circumstances amounts to opportunity formation. Strategic governance might be summed up as what allows for more agency at the individual or community level and mitigates macro pessimism rather than specific institutional drivers (as they will differ by circumstance), or even a prescriptive list of specific governance practices. In these

\footnotetext{
567 Lepenies, 456.

${ }^{568}$ Hirschman, A Bias for Hope: Essays on Development and Latin America, 359.
} 
two case studies where macro conditions were actively conspiring against life and liberty through war and corruption, networks of hope made incremental progress. Strategic governance (by acting as a buffer) was able to interrupt underperforming care at multiple stages to alter outcomes for infants by enhancing the indirect health, and social and policy mechanisms that alleviate the proximate physiological mechanism of infant mortality. Wise discusses the "last mile" of service delivery as an essential component to improve health outcomes in crisis countries ${ }^{569}$. This is undoubtedly true: poor infrastructure, inadequate health facilities must be upgraded or multiplied, delivery care most be enhanced and so forth. This study diverges from the "last mile" theory and suggests that an "entire road" can potentially provide opportunity for significant, sustainable, lifesaving intervention in low resource countries. Strategic governance in these cases comes through a combination of context specific institutional drivers with an emphasis on what strengthens agency at the micro level in the context of these two case studies can reveal conditions that can ease macro-level dysfunction on population health in crisis conditions.

\section{Research findings}

In summarizing the research findings, it appears the cluster of institutional drivers in both ROC and ROY are connected with the indirect health and indirect social and policy mechanisms that can potentially alleviate the proximate physiological

\footnotetext{
569 Wise and Darmstadt, "Strategic Governance.”
} 
mechanisms of infant mortality. The endogenous drivers of decentralization and cultural change supported local and non-state environments to become more opportunistic as exogenous drivers, i.e., international partnerships, funding, and performance-based funding introduced various mechanisms of support and accountability. To make clear the connection between the cluster of practices (institutional drivers) in the two cases and the proximate health and social and policy mechanisms of IMR, an example from each country is summarized below.

\section{Decentralization}

In the case of Congo, decentralization of health care allowed the indirect health mechanism of vaccination programs by UNICEF to work with local level partners, as there was capacity separate from inadequate national administration of health services. Local immunization practices intervened along the chronology of care for women, particularly in the postnatal phase when infants received increased coverage of routine vaccinations and women additional vitamin supplementation. The international vaccination intervention programs at the local level augmented regional capacity. The Yemen case study also highlights the role of decentralization as an institutional driver impacting the indirect health mechanisms of delivery care in the delivery phase in chronology of care for women. Local and regional health facilities were responsible for the provision of care, even particularly hard to reach rural areas that had previously been more or less unattended. Community health workers had to go to the women, rather than women having to find their way to 
health facilities. Building on local capacity and an intentional increase in community health workers, particularly in remote rural areas, scarce resources were made potentially more responsive to local conditions that made a difference in the critical time of delivery. This endogenous institutional driver of decentralization was aligned with global trends towards strengthening local capacity and accountability of health care provision even if the motivation was initially about relieving national responsibility. This move had the compound effect of not only giving agency to local decision makers to address specific local constraints but also to then partner with organizations with specific capacity to address shortcomings.

\section{International partnerships}

The institutional driver of international partnerships in both Congo and Yemen impacted indirect mechanisms to alleviate the proximate physiological mechanisms of infant mortality. For example, Congolese partnerships with the African Union led to addressing maternal mortality at time of delivery as part of an international Campaign for the Accelerated Reduction of Maternal Mortality. The international mission and resulting partnerships facilitated improvement in the indirect health mechanism of improved delivery care through specialized training of doctors to meet emergency care needs. In Yemen, governorate level stakeholders, along with the Maternal and Child Health Integrated Program (a partnership with USAID) shaped indirect social and policy mechanisms of community health workers were 
positively impacted by additional education opportunities that expanded their capacity to serve women and communities.

\section{Funding}

The World Bank through its International Development Association supported the maternal and child health objectives in Congo (as identified in the Millennium Development Goals). With funding, the World Bank filled a critical shortage in the country by providing financial as well as technical assistance for family planning education programs. This financial assistance impacted the critical indirect social and policy mechanisms of health education around family planning in the prenatal phase. Preventing compromised pregnancies improved health outcomes for infants. An example of funding as an institutional driver in Yemen was the Yemen Midwives Association that received financial support from international partnerships including the European Union and the World Health Organization to expand health care coverage in underserved areas. The financial support impacted the indirect health mechanisms of primary care, breastfeeding, vaccines, and delivery, as well as the indirect social and policy mechanisms of community health workers, health education, community interventions, women's health groups, preventative actions and improved health protocols. Growing the cadre of midwives in Yemen had a tremendous impact along the chronology of care for women and funding from multiple sources made it possible. 


\section{Performance-based funding}

The institutional driver of performance-based funding in Congo encouraged the indirect social and policy mechanism of improved health protocols in multiple instances: from assisted births, more thorough vaccination administration, increased number of attended births, number of antenatal visits conducted, hygiene, and respect in the health facility. Funding based on performance had a strong impact that shaped actions throughout the chronology of care and improved infant health outcomes.

\section{Cultural change}

Cultural change in both countries pushed back against long standing traditions of home births. This institutional driver impacted the critical indirect health mechanisms of delivery and vaccinations. In Congo, traditional matrons were previously the accepted attendants at home births (but that scenario is actually considered an unattended birth as the skills of matrons are not consistent with established health protocols for healthy delivery). The shift to facility births became more acceptable and desirable as health care improved. Women learned that there were benefits to facility birth not only for themselves but also for their infants with vaccinations available. Yemen likewise experienced the institutional driver of cultural change as seen in attitudes towards child marriage. International conventions, and growing discontent within the country brought this tradition to the attention of activists trying to end the child marriage practice which could 
prevent unhealthy pregnancies and compromised delivery. Cultural change in this case impacted the indirect health mechanism of delivery that is harder for young women who are not yet physically mature for childbirth.

\section{Strategic governance}

Strategic governance is a form of locally initiated and opportunistic local or community governance that achieves accountable and efficient outcomes through the effective use of social and financial resources. The relative role of endogenous factors is of first importance. Decentralization necessitated local and regional responsibility and accountability to some degree. As such, decentralization of health care in both cases allowed solution seeking at local and regional levels. With increased capacity and responsibility by mandate, local and non-state solutions maximized what was in their power to change. Culture change, another endogenous factor, occurred with varying intensity depending on how much global agendas resonated at local levels and the willingness to course correct based on new information. The increasingly dense interactions through international partnerships impacted cultural change at local levels by opportunities to identify practices that restricted improved health outcomes, like child marriage, or unattended births. Exogenous factors of international change, funding, and performance-based funding support and supplement the primary endogenous factors to enhance capacity. These central and international drivers increase capacity but are of secondary importance after the internal mechanisms. For robust response to central and international intervention, local and regional levels must have some degree of 
autonomy in order to usefully maximize assistance. Without autonomy, exogenous institutional drivers might not have demonstrated the same positive improvements in infant mortality. Local discretion can provide more pointed use of additional resources precisely because particular deficiencies in the region are known.

Implications for Theory

The focus of these "strategic governance" findings is the relationship between macro-conditions and micro-conditions. Local accountability and efficient/equitable health governance can take shape from the bottom-up. The implications of this on the sequencing or dynamics of development is that micro-positive health outcomes are possible even while macro-pessimistic conditions persist. Opportunistic local and regional governments may seek partnerships even before formal authority has been granted from the central government and this can potentially provide fortuitous arrangements but it can also bring scrutiny from the establishment. Based on cultural change and to what degree that change has already taken place in more affluent urban areas, improvements can trickle up to the national level although that was not explicit in this research. Strategic governance can co-exist within unchanged national conditions, particularly in conditions of corruption, as was the case in ROC and ROY. It seems likely given crisis conditions that increased engagement with international partnerships served to not only improve measurable global health priorities and impact culture but demonstrated regime benevolence by "bringing in" assistance to help the underserved while relinquishing responsibility for current and/or future catastrophic health outcomes. A fraudulent regime can 
very much continue a self-serving path and have no compunction for credit claiming when indicated for successful health outcomes based on local efforts and practical partnerships. Over time macro-pessimistic conditions could change but it is not a pre-cursor to development impact.

One might ask if cultural change is a macro-structural constraint rather than an institutional driver. If one considers "culture" a monolithic influence over an entire country then it might well be grouped with the other macro-structural factors that hang together like democracy, economic growth and governance. On the other hand, if one imagines culture to be more sensitive to environment then it seems far more likely that culture is not the monolith as thought. Culture in the contexts of ROC and ROY was very specific to many different small pockets of communities. Urban and rural areas had vastly different experiences and unequal access to resources of water, housing, education, information, infrastructure and health care. Addressing the cultural constraints in locally appropriate ways (through participation in women's groups, community health workers, or women's health education for instance) has the potential to modify those traditional convictions that impair positive health outcomes. In view of the cultural change in the case study countries it appears there is room to consider culture as local phenomena, or subnational condition, rather than a macro-constraint.

Implications for Policy and Practice

In light of the research and theory findings, policy and practice could potentially benefit from six considerations: foreign aid works, decentralization works, targeted 
fiscal resources work, resource allocation and management is as important as resource level, culture matters cannot be ignored, and central government should not be by-passed but "rebuilt from the bottom up" through such interventions as these. First, simply put foreign aid works in the direst conditions where it can bypass crippling government dysfunction and corruption. Aid can almost immediately alleviate suffering through lifesaving vaccines and perhaps more importantly over time through support and leadership in education. In many cases, foreign aid in the form of funding for educators as seen in ROY where midwives were trained and they subsequently trained more midwives is the foundation for endogenous change and micro agency for health care improvement. This aid of educational support is equally valuable with vaccination programs and while it might take more time to see the benefit, it is liberating for communities to have capacity to address local health concerns. Second, decentralization of health care to the local level allows responsiveness to specific needs whether through increased education regarding the vertical transmission of HIV through breastfeeding to education about the value of facility births. Decentralization of health care places the responsibility for accountability and equity closer to the communities served. Responsiveness to community need seems a more likely outcome in a local context. Third, targeted fiscal resources work, particularly where spending is low or nonexistent. Resources allocated for necessities like hospital beds, and for women unable to afford caesareans can make significant and immediate impacts as in ROC. Fourth, the management and allocation of resources at appropriate levels is 
essential. In order to maximize limited resources, as in ROC, performance-based funding reduced infant mortality by incentivizing measurable outcomes that aligned with best health care practices like the number of children fully immunized or the number of facility births. Linking funding to established health protocols or "best practices" allied administration and health care providers with clear paths for health care implementation strategies. Fifth, from the beginning, the importance of education, information, gender norms, and cultural practices must be part of the development program planning processes. Cultural change should not be forced or demanded but when prescribed it should be in the context of local environments. Promoting facility birth in rural Yemen could prove a waste of resources if women are not allowed to leave their home, or if it is a geographical impossibility because health clinics are too far away. Instead, awareness of cultural conditions should form the creative basis of how health care can respond to meet community health needs, as in Yemen where CHWs visit pregnant women in their homes. Finally, in the process of planning, central governments should not be by-passed but "rebuilt from the bottom up" through such interventions as these. Improving health outcomes and governance over time, notably at local levels, took place in both ROC and ROY because as seen IMR improved in macro-pessimistic conditions. To by-pass the opportunity for the same in central governments would be to the detriment of future generations. 
Future Research Agenda

Continuing the exploration of strategic governance might offer additional insight on how developing countries can alleviate negative health outcomes and provide more examples of what works, what does not and policy implications for international aid organizations. As such, future research on the role of strategic governance in crisis conditions might extend to six considerations: non-IMR development outcomes, updates on the ROC and ROY cases since 2014 to see if even worse macro-structural conditions in the latter make strategic governance impossible, extension to the counterfactual "lower left quadrant" cases with poor macro conditions and below average IMR to see if there is an absence of strategic governance components is evident, extension of case country study in the "top left quadrant" of cases with poor macro conditions but better than average IMR, sub-national studies within countries, and the consideration of cultural change as a macro-constraint as potentially relevant. First, other non-IMR development outcomes can be explored to broaden the discussion within the countries included in this case study as well as others to see if there are similar or conflicting results. Second, updating the ROC and ROY cases since 2014 to reflect worsening macro-structural constraints (particularly in ROY) might demonstrate if strategic governance was able to accommodate increased pessimism (or not) while still improving IMR. Maybe there is a threshold in governance indicators across which intermediate strategic governance factors are not able to intercede to better IMR if certain institutional drivers are not present (see Mauritania and Central African Republic in Figure 5 the 
"lower left quadrant"). Third, along the same line, research could extend to the lower left quadrant to see what if any, additional macro-structural constraints are present, and what historical or current events might prevent IMR improvement. ROC and ROY both experienced war but perhaps there is something unique in the lower left quadrant that prevented strategic governance from helping micro levels improve health outcomes. Forth, the "top left quadrant" provides further opportunity to explore similar cases with bad macro structural conditions but good IMR. Fifth, within all cases, subnational studies should be considered another research area to lend thicker detail to strategic governance findings. Finally, the cultural component might be another relevant area to explore in terms of its relative importance in certain cases at the macro-structural level or not. 


\section{References}

Abrahams, Zulfa, Zandile McHiza, and Nelia P Steyn. "Diet and Mortality Rates in Sub-Saharan Africa: Stages in the Nutrition Transition." BMC Public Health 11 (October 13, 2011): 801-801. https://doi.org/10.1186/1471-2458-11-801.

Adelman, Jeremy. Worldly Philosopher The Odyssey of Albert O. Hirschman. Princeton, N.J.: Princeton University Press, 2013.

Adeyi, Olusoji, Gnanaraj Chellaraj, Ellen Goldstein, Alexander Preker, and Dena Ringold. "Health Status during the Transition in Central and Eastern Europe: Development in Reverse?" Health Policy and Planning 12, no. 2 (January 1, 1997): 132-45. https://doi.org/10.1093/heapol/12.2.132.

Akena, Charles. "Maternal Mortality down Sharply in Congo." The New Humanitarian, January 21, 2013. http://www.thenewhumanitarian.org/report/97300/maternal-mortalitydown-sharply-congo.

Al Serouri, Abdul Wahed, Arwa Al Rabee, Mohammed Bin Afif, and Abdullah Al Rukeimi. "Reducing Maternal Mortality in Yemen: Challenges and Lessons Learned from Baseline Assessment." International Journal of Gynecology \& Obstetrics 105, no. 1 (2009): 86-91.

Al Serouri, Abdul Wahed, Abdulla Al Rukeimi, Mohammed Bin Afif, Abdulrahman Al Zoberi, Jamela Al Raeby, Catherine Briggs, and Hamouda Hanafi. "Findings from a Needs Assessment of Public Sector Emergency Obstetric and Neonatal 
Care in Four Governorates in Yemen: A Human Resources Crisis." Reproductive Health Matters 20, no. 40 (2012): 122-128.

Al-Taiar, Abdullah, Allan Clark, Joseph C. Longenecker, and Christopher Jm Whitty. "Physical Accessibility and Utilization of Health Services in Yemen." International Journal of Health Geographics 9, no. 1 (2010): 38-38. https://doi.org/10.1186/1476-072X-9-38.

Amonoo-Lartson, R. District Health Care: Challenges for Planning, Organisation, and Evaluation in Developing Countries. London: Macmillan Press, 1984.

Anand, Sudhir, and Martin Ravallion. "Human Development in Poor Countries: On the Role of Private Incomes and Public Services." Journal of Economic Perspectives 7, no. 1 (March 1993): 133-50. https://doi.org/10.1257/jep.7.1.133.

Arevshatian, L., C. J. Clements, S. K. Lwanga, A. O. Misore, P. Ndumbe, J. F. Seward, and P. Taylor. "An Evaluation of Infant Immunization in Africa: Is a Transformation in Progress?" Bulletin of the World Health Organization 85 (2007): 449-457.

Azfar, Omar, Jeffrey Livingston, and Patrick Meagher. "Decentralization in Uganda." Decentralization and Local Governance in Developing Countries: A Comparative Perspective, 2006, 223-257.

Bahl, Rajiv, Shamim Qazi, Gary L. Darmstadt, and Jose Martines. "Why Is Continuum of Care from Home to Health Facilities Essential to Improve Perinatal 
Survival?" Seminars in Perinatology 34, no. 6 (December 2010): 477-85. https://doi.org/10.1053/j.semperi.2010.09.001.

Baldacci, Emanuele, Benedict Clements, Sanjeev Gupta, and Qiang Cui. “Social Spending, Human Capital, and Growth in Developing Countries." World Development 36, no. 8 (2008): 1317-1341.

Ballard, Karen, Zelalem Belete, Hirut Kinfu, Mebkyou Tadesse, Mohammed Amin, and Habtamu Atnafu. "The Effect of Prenatal and Intrapartum Care on the Stillbirth Rate among Women in Rural Ethiopia." International Journal of Gynecology \& Obstetrics 133, no. 2 (2016): 164-167.

Banajeh, S. M., A. M. Al Rabee, and I. H. Al Arashi. "Burden of Perinatal Conditions in Yemen: A 12-Year Hospital-Based Study," 2005.

Bang, Abhay T, Hanimi M Reddy, Rani A Bang, and Mahesh D Deshmukh. “Why Do Neonates Die in Rural Gadchiroli, India? (Part II): Estimating Population Attributable Risks and Contribution of Multiple Morbidities for Identifying a Strategy to Prevent Deaths." Journal of Perinatology 25, no. S1 (March 2005): S35-43. https://doi.org/10.1038/sj.jp.7211270.

Bardhan, Pranab K., and Dilip Mookherjee. Decentralization and Local Governance in Developing Countries: A Comparative Perspective. Cambridge, Mass.: MIT Press, 2006.

Basaleem, H. O. “Women's Reproductive Health Seeking Behavior in Four Districts in Sana'a, Yemen: Quantitative and Qualitative Analysis." Journal of Community Medicine and Health Education 2, no. 153 (2012): 2161-0711. 
Bates, Robert H., Avner Greif, Margaret Levi, Jean-Laurent Rosenthal, Barry R. Weingast, and Barry R. Weingast. Analytic Narratives. 1st US Edition 1st Printing edition. Princeton, N.J: Princeton University Press, 1998.

Bazzano, Alessandra, Zelee Hill, Charlotte Tawiah-Agyemang, Alexander Manu, Guus ten Asbroek, and Betty Kirkwood. "Introducing Home Based Skin-to-Skin Care for Low Birth Weight Newborns: A Pilot Approach to Education and Counseling in Ghana." Global Health Promotion 19, no. 3 (2012): 42-49.

Besley, Timothy, and Masayuki Kudamatsu. "Health and Democracy." In The American Economic Review., 96:313-18, 2006. https://doi.org/10.1257/000282806777212053.

Bhutta, Zulfiqar A., Sergio Cabral, Chok-Wan Chan, and William J. Keenan. "Reducing Maternal, Newborn, and Infant Mortality Globally: An Integrated Action Agenda." International Journal of Gynecology \& Obstetrics 119, no. 1 (2012): S13-S17. https://doi.org/10.1016/j.ijgo.2012.04.001.

Bhutta, Zulfiqar A., Gary L. Darmstadt, Babar S. Hasan, and Rachel A. Haws. “Community-Based Interventions for Improving Perinatal and Neonatal Health Outcomes in Developing Countries: A Review of the Evidence." Pediatrics 115, no. 2 Suppl (2005): 519-617. https://doi.org/10.1542/peds.2004-1441.

Bhutta, Zulfiqar A, Jai K Das, Rajiv Bahl, Joy E Lawn, Rehana A Salam, Vinod K Paul, M Jeeva Sankar, et al. "Can Available Interventions End Preventable Deaths in Mothers, Newborn Babies, and Stillbirths, and at What Cost?" The Lancet 384, 
no. 9940 (July 2014): 347-70. https://doi.org/10.1016/S01406736(14)60792-3.

Binder, Henry J., Ian Brown, B. S. Ramakrishna, and Graeme P. Young. “Oral Rehydration Therapy in the Second Decade of the Twenty-First Century." Current Gastroenterology Reports 16, no. 3 (February 22, 2014): 376. https://doi.org/10.1007/s11894-014-0376-2.

Bitar, Salwa. "Accelerating the Spread of Best Practices in Postpartum Care: ScalingUp Best Practices in Yemen." IntraHealth, 2010. https://www.intrahealth.org/resources/accelerating-spread-best-practicespostpartum-care-scaling-best-practices-yemen.

Black, Robert E, Saul S Morris, and Jennifer Bryce. “Where and Why Are 10 Million Children Dying Every Year?" The Lancet 361, no. 9376 (June 28, 2003): 222634. https://doi.org/10.1016/S0140-6736(03)13779-8.

Blumi, Isa. Destroying Yemen: What Chaos in Arabia Tells Us about the World. Oakland, California: University of California Press, 2018.

Boix, Carles. "Democracy, Development, and the Public Sector." American Journal of Political Science, 2001, 1-17.

Boyle, Michael H., Yvonne Racine, Katholiki Georgiades, Dana Snelling, Sungjin Hong, Walter Omariba, Patricia Hurley, and Purnima Rao-Melacini. “The Influence of Economic Development Level, Household Wealth and Maternal Education on Child Health in the Developing World." Social Science \& Medicine 63, no. 8 (2006): 2242-2254. 
Brandt, Marieke. Tribes and Politics in Yemen: A History of the Houthi Conflict. New York]: Oxford University Press, 2017. https://www.oxfordscholarship.com/view/10.1093/oso/9780190673598.0 01.0001/oso-9780190673598.

Brown, David S., and Wendy Hunter. "Democracy and Human Capital Formation: Education Spending in Latin America, 1980 to 1997." Comparative Political Studies 37, no. 7 (2004): 842-864.

Brown, David S., and Ahmed Mushfiq Mobarak. "The Transforming Power of Democracy: Regime Type and the Distribution of Electricity." American Political Science Review 103, no. 2 (2009): 193-213.

Brown, David W., Anthony Burton, Marta Gacic-Dobo, Rouslan I. Karimov, Jos Vandelaer, and Jean Marie Okwo-Bele. “A Mid-Term Assessment of Progress towards the Immunization Coverage Goal of the Global Immunization Vision and Strategy (GIVS)." BMC Public Health 11, no. 1 (2011): 806-806. https://doi.org/10.1186/1471-2458-11-806.

Bukair, A., A. W. Al-Saqladi, and A. Al-Sadeeq. "Interpregnancy Interval and the Risk of Preterm Birth: A Case-Control Study of Infants Born at Al-Sadaqa General Teaching Hospital, Aden, Yemen." International Journal of Reproduction, Contraception, Obstetrics and Gynecology 5, no. 4 (2016): 1181-1186.

Busulwa, R., M. Y. Takiyaddin, A. A. Azzubeidi, El Mousaad, H. El Zein, J. Tawillah, and H. Ziady. "Perceptions of the Condom as a Method of HIV Prevention in Yemen," 2006. 
Busygina, Irina, Mikhail Filippov, and Elmira Taukebaeva. "To Decentralize or to Continue on the Centralization Track: The Cases of Authoritarian Regimes in Russia and Kazakhstan." Journal of Eurasian Studies 9, no. 1 (2018): 61-71. https://doi.org/10.1016/j.euras.2017.12.008.

Campbell, Shelagh. "Comparative Case Study." In Encyclopedia of Case Study Reserach, 175-76. Thousand Oaks: SAGE Publications, Inc, 2012.

Carapico, Sheila. Civil Society in Yemen the Political Economy of Activism in Modern Arabia. Cambridge Middle East Studies 9. Cambridge, U.K. ; New York: Cambridge University Press, 1998.

Central Statistical Organizaton (CSO), and Macro International. "Yemen Demographic and Maternal and Child Health Survey 1997," November 1, 1998. https://dhsprogram.com/publications/publication-FR94-DHS-FinalReports.cfm.

Chao, Shin, Giannina Donatoni, Cathleen Bemis, Kevin Donovan, Cynthia Harding, Deborah Davenport, Carol Gilbert, Laurin Kasehagen, and Magda Peck. “Integrated Approaches to Improve Birth Outcomes: Perinatal Periods of Risk, Infant Mortality Review, and the Los Angeles Mommy and Baby Project." Maternal and Child Health Journal 14, no. 6 (2010): 827-837. https://doi.org/10.1007/s10995-010-0627-2.

Chiwuzie, Jasper, and Chike Okolocha. "Traditional Belief Systems and Maternal Mortality in a Semi-Urban Community in Southern Nigeria." African Journal of 
Reproductive Health / La Revue Africaine de La Santé Reproductive 5, no. 1 (2001): 75-82. https://doi.org/10.2307/3583200.

CIA. “The World Factbook.” CIA. Accessed November 20, 2019.

https://www.cia.gov/library/publications/the-world-factbook/geos/cf.html.

Clark, John F. John Frank. The Failure of Democracy in the Republic of Congo. Boulder, Colo.: Lynne Rienner Publishers, 2008.

CNSEE/Congo, Centre National de la Statistique et des Études Économiques-, and I.

C. F. International. “Congo Enquête Démographique et de Santé 2011-2012,"

December 1, 2012. https://dhsprogram.com/publications/publicationFR267-DHS-Final-Reports.cfm.

CNSEE/Congo, Centre National de la Statistique et des Études Économiques-, and O.

R. C. Macro. “Congo Enquête Démographique et de Santé 2005," July 1, 2006. https://dhsprogram.com/publications/publication-FR182-DHS-FinalReports.cfm.

Colburn, Marta. The Dynamic of Development and Democratization in Yemen. Bonn: Friedrich-Ebert-Stiftung, 2002.

- - . The Republic of Yemen: Development Challenges in the 21st Century. London: Stacey International, 2002.

Collier, David. “Understanding Process Tracing." PS: Political Science \& Politics 44, no. 04 (October 2011): 823-30.

https://doi.org/10.1017/S1049096511001429. 
Colton, Nora Ann. "Yemen: A Collapsed Economy." The Middle East Journal;

Washington 64, no. 3 (Summer 2010): 410-26.

http://dx.doi.org.proxy.lib.pdx.edu/10.3751/64.3.14.

Compagni, Amelia, and Fabrizio Tediosi. "Implementing Performance-Based

Funding for Health Research: When Governance and Procedural Fairness

Matter." Public Administration 90, no. 2 (2012): 313-34.

https://doi.org/10.1111/j.1467-9299.2011.01965.x.

“Congo | UNAIDS." Accessed November 25, 2019.

https://www.unaids.org/en/regionscountries/countries/congo.

“Constitute." Accessed October 21, 2019.

https://www.constituteproject.org/constitution/Congo_2015?lang=en.

Cordaid. "Healthcare Approach Successful in Congo Brazzaville." Cordaid, 2013. https://www.google.com/search?client=safari\&rls=en\&q=healthcare+appro ach+successful+in+congo+brazzaville\&ie=UTF-8\&oe=UTF- 8 .

Cramer, James C. "Social Factors and Infant Mortality: Identifying High-Risk Groups and Proximate Causes." Demography 24, no. 3 (August 1987): 299-322.

Cutler, David M., and Adriana Lleras-Muney. "Education and Health: Evaluating Theories and Evidence." National bureau of economic research, 2006.

Dadelszen, Peter von, Laura A. Magee, Beth A. Payne, Dustin T. Dunsmuir, Sharla Drebit, Guy A. Dumont, Suellen Miller, et al. “Moving beyond Silos: How Do We Provide Distributed Personalized Medicine to Pregnant Women Everywhere at Scale? Insights from PRE-EMPT." International Journal of 
Gynecology \& Obstetrics, World Report on Women's Health 2015: The unfinished agenda of women's reproductive health, 131 (October 1, 2015): S10-15. https://doi.org/10.1016/j.ijgo.2015.02.008.

Darmstadt, Gary L, Zulfiqar A Bhutta, Simon Cousens, Taghreed Adam, Neff Walker, and Luc de Bernis. "Evidence-Based, Cost-Effective Interventions: How Many Newborn Babies Can We Save?" The Lancet 365, no. 9463 (March 18, 2005): 977-88. https://doi.org/10.1016/S0140-6736(05)71088-6.

Darmstadt, Gary L., Anne CC Lee, Simon Cousens, Lynn Sibley, Zulfiqar A. Bhutta, France Donnay, Dave Osrin, et al. "60 Million Non-Facility Births: Who Can Deliver in Community Settings to Reduce Intrapartum-Related Deaths?" International Journal of Gynecology \& Obstetrics 107, no. Supplement (October 2009): S89-112. https://doi.org/10.1016/j.ijgo.2009.07.010.

_—_. "60million Non-Facility Births: Who Can Deliver in Community Settings to Reduce Intrapartum-Related Deaths?" International Journal of Gynecology \& Obstetrics, Intrapartum-Related Deaths: Evidence for Action, 107 (October 1, 2009): S89-112. https://doi.org/10.1016/j.ijgo.2009.07.010.

Darmstadt, Gary L, Tanya Marchant, Mariam Claeson, Win Brown, Saul Morris, France Donnay, Mary Taylor, et al. “A Strategy for Reducing Maternal and Newborn Deaths by 2015 and Beyond." BMC Pregnancy and Childbirth 13, no. 1 (December 2013). https://doi.org/10.1186/1471-2393-13-216. 
Deaton, Angus. “Global Patterns of Income and Health: Facts, Interpretations, and Policies." National Bureau of Economic Research, 2006. http://www.nber.org.proxy.lib.pdx.edu/papers/w12735.

Deller, Barbara, Vandana Tripathi, Stacie Stender, Emmanuel Otolorin, Peter Johnson, and Catherine Carr. "Task Shifting in Maternal and Newborn Health Care: Key Components from Policy to Implementation." International Journal of Gynecology \& Obstetrics, Advancing Implementation in Maternal and Newborn Health, 130 (June 1, 2015): S25-31. https://doi.org/10.1016/j.ijgo.2015.03.005.

Demicheli, Vittorio, Antonella Barale, and Alessandro Rivetti. "Vaccines for Women for Preventing Neonatal Tetanus." Cochrane Database of Systematic Reviews, no. 7 (2015). https://doi.org/10.1002/14651858.CD002959.pub4.

Diamond, Larry. "The Rule of Law versus the Big Man 1." In In Search of Democracy, 252-263. Routledge, 2015.

Dingli, Sophia. "Is the Failed State Thesis Analytically Useful? The Case of Yemen." Politics 33, no. 2 (2013): 91-100. https://doi.org/10.1111/j.14679256.2012.01453.x.

Doherty, Tanya, David Sanders, Ameena Goga, and Debra Jackson. "Implications of the New WHO Guidelines on HIV and Infant Feeding for Child Survival in South Africa." Bulletin Of The World Health Organization 89, no. 1 (January 1, 2011): 62-67. https://doi.org/10.2471/BLT.10.079798.

Dunn, William N. Public Policy Analysis. 5 edition. Boston: Routledge, 2011. 
Edmond, Karen M., Maria A. Quigley, Charles Zandoh, Samuel Danso, Chris Hurt, Seth Owusu Agyei, and Betty R. Kirkwood. "Aetiology of Stillbirths and Neonatal Deaths in Rural Ghana: Implications for Health Programming in Developing Countries." Paediatric \& Perinatal Epidemiology 22, no. 5 (September 2008): 430-37. https://doi.org/10.1111/j.1365-3016.2008.00961.x.

"Embassy of the Republic of Congo in Washington DC > About Congo > Government." Accessed October 14, 2019. http://www.ambacongous.org/en-us/aboutcongo/government.aspx.

European Commission. "Yemen- European Community Strategy Paper for the Period 2007-2013," n.d.

Filmer, Deon, and Lant Pritchett. "The Impact of Public Spending on Health: Does Money Matter?" Social Science \& Medicine 49, no. 10 (1999): 1309-1323.

Fink, Günther, Rebecca Ross, and Kenneth Hill. "Institutional Deliveries Weakly Associated with Improved Neonatal Survival in Developing Countries: Evidence from 192 Demographic and Health Surveys." International Journal of Epidemiology 44, no. 6 (December 1, 2015): 1879-88. https://doi.org/10.1093/ije/dyv115.

Freedom House. "Congo, Republic of (Brazzaville)." Freedom in the World 2019, January 28, 2019. https://freedomhouse.org/report/freedomworld/2019/congo-republic-brazzaville. 
———. "Freedom in the World: Congo, Brazzaville 2009." Freedom House, n.d. https://freedomhouse.org/report/freedom-world/2009/congo-republicbrazzaville.

Frère, Marie-Soleil. "Covering Post-Conflict Elections: Challenges for the Media in Central Africa." Africa Spectrum 46, no. 1 (2011): 3-32.

Gakidou, Emmanuela, Krycia Cowling, Rafael Lozano, and Christopher JL Murray. "Increased Educational Attainment and Its Effect on Child Mortality in 175 Countries between 1970 and 2009: A Systematic Analysis." The Lancet 376, no. 9745 (2010): 959-974.

Gauri, Varun, and Peyvand Khaleghian. "Immunization in Developing Countries: Its Political and Organizational Determinants." World Development 30, no. 12 (2002): 2109-2132.

Geertz, Clifford. "Thick Description: Toward an Interpretive Theory of Culture." Readings in the Philosophy of Social Science, 1994, 213-231.

Gerring, John, Peter Kingstone, Matthew Lange, and Aseema Sinha. "Democracy, History, and Economic Performance: A Case-Study Approach." World Development 39, no. 10 (October 2011): 1735-48. https://doi.org/10.1016/j.worlddev.2011.04.029.

Gerring, John, Strom Thacker, and Rodrigo Alfaro. "Democracy and Human Development." The Journal of Politics 74, no. 1 (January 2012): 1-17. Ghadrshenas, Anisa, Yanis B. Amor, Joy Chang, Helen Dale, Gayle Sherman, Lara Vojnov, Paul Young, et al. "Improved Access to Early Infant Diagnosis Is a 
Critical Part of a Child-Centric Prevention of Mother-to-Child Transmission Agenda." Aids 27 (2013): S197-S205.

Ghandour, Reem M, Katherine Flaherty, Ashley Hirai, Vanessa Lee, Deborah Klein Walker, and Michael C Lu. "Applying Collaborative Learning and Quality Improvement to Public Health: Lessons from the Collaborative Improvement and Innovation Network (CoIIN) to Reduce Infant Mortality." Maternal And Child Health Journal 21, no. 6 (June 2017): 1318-26. https://doi.org/10.1007/s10995-016-2235-2.

Ghobarah, Hazem Adam, Paul Huth, and Bruce Russett. "Comparative Public Health: The Political Economy of Human Misery and Well-Being." International Studies Quarterly 48, no. 1 (2004): 73-94.

"Global Strategy: Breastfeeding Critical for Child Survival--UNICEF and WHO Call for Increased Commitment to Appropriate Feeding Practices for All Infants and Young Children." Indian Journal of Medical Sciences 58, no. 3 (March 2004): 138-39.

Goodburn, Elizabeth A., Rukhsana Gazi, and Mushtaque Chowdhury. "Beliefs and Practices Regarding Delivery and Postpartum Maternal Morbidity in Rural Bangladesh." Studies in Family Planning 26, no. 1 (1995): 22-32. https://doi.org/10.2307/2138048.

“Governance for Sustainable Human Development." UNDP Report, 1997. 
Great Britain. Naval Intelligence Division. French Equatorial Africa \& Cameroons. Great Britain. Naval Intelligence Division. Geographical Handbook Series. London, 1942.

Grindle, Merilee S. “Good Enough Governance Revisited.” Development Policy Review 25, no. 5 (September 1, 2007): 533-74. https://doi.org/10.1111/j.14677679.2007.00385.x.

Guadagno, Marie, Michael Mackert, and Aaron Rochlen. "Improving Prenatal Health: Setting the Agenda for Increased Male Involvement." American Journal of Men's Health 7, no. 6 (2013): 523-526. https://doi.org/10.1177/1557988313490785.

Gupta, Sanjeev, Marijn Verhoeven, and Erwin R. Tiongson. "The Effectiveness of Government Spending on Education and Health Care in Developing and Transition Economies." European Journal of Political Economy 18, no. 4 (2002): 717-737.

Haggblade, Steven. "Successes in African Agriculture: Building for the Future." In Findings of an International Conference, Pretoria, South Africa.(December 1-3, 2003). InWent, IFPRI, NEPAD, CTA, 2004.

Halleröd, Björn, Bo Rothstein, Adel Daoud, and Shailen Nandy. "Bad Governance and Poor Children: A Comparative Analysis of Government Efficiency and Severe Child Deprivation in 68 Low- and Middle-Income Countries." World Development 48 (August 2013): 19-31. https://doi.org/10.1016/j.worlddev.2013.03.007. 
Hanmer, Lucia, Robert Lensink, and Howard White. "Infant and Child Mortality in Developing Countries: Analysing the Data for Robust Determinants." The Journal of Development Studies 40, no. 1 (2003): 101-118.

Haver, Jaime, William Brieger, Jérémie Zoungrana, Nasratullah Ansari, and Jean Kagoma. “Experiences Engaging Community Health Workers to Provide Maternal and Newborn Health Services: Implementation of Four Programs." International Journal of Gynecology \& Obstetrics, Advancing Implementation in Maternal and Newborn Health, 130 (June 1, 2015): S32-39. https://doi.org/10.1016/j.ijgo.2015.03.006.

Hirschman, Albert O. A Bias for Hope: Essays on Development and Latin America. New Haven: Yale University Press, 1971.

Hodge, Graeme A., and Carsten Greve. "Public-Private Partnerships: An International Performance Review." Public Administration Review 67, no. 3 (2007): 545-558.

Holst, Jens, and Christian A. Gericke. “Healthcare Financing in Yemen.” International Journal of Health Planning and Management 27, no. 3 (2012): 198-225. https://doi.org/10.1002/hpm.2107.

Hoope-Bender, Petra ten, Luc de Bernis, James Campbell, Soo Downe, Vincent Fauveau, Helga Fogstad, Caroline SE Homer, Holly Powell Kennedy, Zoe Matthews, and Alison McFadden. "Improvement of Maternal and Newborn Health through Midwifery." The Lancet 384, no. 9949 (2014): 1226-1235. 
Hu, Bingjie, and Ronald U. Mendoza. "Public Health Spending, Governance and Child Health Outcomes: Revisiting the Links." Journal of Human Development and Capabilities 14, no. 2 (May 1, 2013): 285-311. https://doi.org/10.1080/19452829.2013.765392.

Huffman, Sandra L., Elizabeth R. Zehner, and Cesar Victora. "Can Improvements in Breast-Feeding Practices Reduce Neonatal Mortality in Developing Countries?" Midwifery 17, no. 2 (June 1, 2001): 80-92. https://doi.org/10.1054/midw.2001.0253.

“Human Development Index (HDI) | Human Development Reports." Accessed September 27, 2019. http://hdr.undp.org/en/content/human-developmentindex-hdi.

“ICL - Congo-Brazzaville - Constitution.” Accessed October 21, 2019. http://www.servat.unibe.ch/icl/cf00000_html.

Institut National de la Statistique et UNICEF. "Enquête Par Grappes à Indicateurs Multiples (MICS5 2014-2015), Rapport Final." Brazzaville, Congo: Institut National de la Statistique et UNICEF, 2015.

Issah, Kofi, Alexis Nang-Beifubah, and Chris F. Opoku. "Maternal and Neonatal Survival and Mortality in the Upper West Region of Ghana." International Journal of Gynecology \& Obstetrics 113, no. 3 (June 1, 2011): 208-10. https://doi.org/10.1016/j.ijgo.2011.01.007.

Issaka, Abukari, Kingsley Agho, Andrew Page, Penelope Burns, Garry Stevens, and Michael Dibley. "Factors Associated with Early Introduction of Formula 
and/or Solid, Semi-Solid or Soft Foods in Seven Francophone West African Countries." Nutrients 7, no. 2 (2015): 948-969.

Jahan, S., and S. Jahan. "Evidence-Based Public Health Policy and Practice: Poverty and Infant Mortality in the Eastern Mediterranean Region: A Meta-Analysis." Journal of Epidemiology and Community Health (1979-) 62, no. 8 (2008): 745751.

Jiménez-Rubio, Dolores, and Pilar García-Gómez. "Decentralization of Health Care Systems and Health Outcomes: Evidence from a Natural Experiment." Social Science \& Medicine 188 (2017): 69-81. https://doi.org/10.1016/j.socscimed.2017.06.041.

Jones, Sam, and Inge Tvedten. "What Does It Mean to Be Poor? Investigating the Qualitative-Quantitative Divide in Mozambique." World Development 117 (2019): 153-166.

Jones, Todd. "Interpretive Social Science and the" Native's Point of View": A Closer Look." Philosophy of the Social Sciences 28, no. 1 (1998): 32-68.

Kanyenze, Godfrey, Alternatives to Neo-Liberalism in Southern Africa, Labour \& Economic Development Research Institute Zimbabwe, and Zimbabwe Congress of Trade Unions. Beyond the Enclave towards a Pro-Poor and Inclusive Development Strategy for Zimbabwe. Harare: Weaver Press in association with Alternatives to Neo-liberalism in Southern Africa, Labour \& Economic Development Research Institute, Zimbabwe and the Zimbabwe Congress of Trade Unions, 2011. 
Kempe, Annica, Töres Theorell, Fatoom Noor-Aldin Alwazer, Kyllike Christensson, and Annika Johansson. “Yemeni Women's Perceptions of Own Authority during Childbirth: What Does It Have to Do with Achieving the Millennium Development Goals?" Midwifery 29, no. 10 (2013): 1182-1189.

Kesselman, J. “Private Midwives Serve the Hard-to-Reach: A Promising Practice Model." IntraHealth, 2010. https://www.intrahealth.org/sites/ihweb/files/attachmentfiles/ESD_Legacy_Yemen_Private_Midwives_6_24_10.pdf.

Khalife, Nadya. "How Come You Allow Little Girls to Get Married? Child Marriage in Yemen.," 2011.

Khan, Qaiser, and Susan Chase. Yemen and the Millennium Development Goals. World Bank, 2003.

Khanani, Intisar, Jon Elam, Rick Hearn, Camille Jones, and Noble Maseru. "The Impact of Prenatal WIC Participation on Infant Mortality and Racial Disparities." American Journal of Public Health 100, no. S1 (April 2, 2010): S204. https://doi.org/10.2105/AJPH.2009.168922.

Kouanda, Seni, Aristide Bado, Ivlabèhiré Bertrand Meda, Gisèle S. Yameogo, Abou Coulibaly, and Slim Haddad. "Home Births in the Context of Free Health Care: The Case of Kaya Health District in Burkina Faso." International Journal of Gynecology \& Obstetrics, Maternal and Neonatal Health in Africa at MDG End: Availability of and Access to Maternal Health Services, and Outcomes of 
Intervention Strategies, 135 (November 1, 2016): S39-44.

https://doi.org/10.1016/j.ijgo.2016.08.009.

Kruk, Margaret Elizabeth, Denis Porignon, Peter C. Rockers, and Wim Van Lerberghe. "The Contribution of Primary Care to Health and Health Systems in Low- and Middle-Income Countries: A Critical Review of Major Primary Care Initiatives." Social Science \& Medicine 70, no. 6 (March 1, 2010): 904-11. https://doi.org/10.1016/j.socscimed.2009.11.025.

Kudamatsu, Masayuki. "Has Democratization Reduced Infant Mortality in SubSaharan Africa? Evidence from Micro Data." Journal of the European Economic Association 10(6) (December 2012): 1294-1317.

Lackner, Helen. Yemen in Crisis: Road to War. Verso Books, 2019.

Lake, David A., and Matthew A. Baum. "The Invisible Hand of Democracy: Political Control and the Provision of Public Services." Comparative Political Studies 34, no. 6 (2001): 587-621.

Lallemant, Camille, Gaston Halembokaka, Gaelle Baty, Nicole Ngo-Giang-Huong, Francis Barin, and Sophie Le Coeur. "Impact of HIV/Aids on Child Mortality before the Highly Active Antiretroviral Therapy Era: A Study in Pointe-Noire, Republic of Congo." Journal of Tropical Medicine 2010 (2010). https://doi.org/10.1155/2010/897176.

Lancet. "Water with Sugar and Salt." The Lancet 312, no. 8.084 (1978). 
Larwanou, M., M. Abdoulaye, and C. Reij. “Etude de La Régénération Naturelle Assistée Dans La Région de Zinder (Niger)." Washington, DC: International Resources Group, 2006.

Lassi, Zohra S., and Zulfiqar A. Bhutta. "Community-Based Intervention Packages for Reducing Maternal and Neonatal Morbidity and Mortality and Improving Neonatal Outcomes." Cochrane Database of Systematic Reviews, no. 3 (2015).

Lawn, JE, K Kerber, A Enweronu-Laryea, and O Massee Bateman. “Newborn Survival in Low Resource Settings-Are We Delivering?" BJOG An International Journal of Obstetrics and Gynaecology 116 (2009): 49-59.

Lawn, Joy E, Hannah Blencowe, Shefali Oza, Danzhen You, Anne CC Lee, Peter Waiswa, Marek Lalli, et al. “Every Newborn: Progress, Priorities, and Potential beyond Survival." The Lancet 384, no. 9938 (July 2014): 189-205. https://doi.org/10.1016/S0140-6736(14)60496-7.

Lawn, Joy E, Simon Cousens, and Jelka Zupan. "4 Million Neonatal Deaths: When? Where? Why?" Lancet (London, England) 365, no. 9462 (March 5, 2005): 891-900.

Lawn, Joy E., Mary Kinney, Anne CC Lee, Mickey Chopra, France Donnay, Vinod K. Paul, Zulfiqar A. Bhutta, Massee Bateman, and Gary L. Darmstadt. "Reducing Intrapartum-Related Deaths and Disability: Can the Health System Deliver?" International Journal of Gynecology \& Obstetrics 107, no. Supplement (2009). Lawn, Joy E., Anne CC Lee, Mary Kinney, Lynn Sibley, Wally A. Carlo, Vinod K. Paul, Robert Pattinson, and Gary L. Darmstadt. “Two Million Intrapartum-Related 
Stillbirths and Neonatal Deaths: Where, Why, and What Can Be Done?" International Journal of Gynecology \& Obstetrics, Intrapartum-Related Deaths: Evidence for Action, 107 (October 1, 2009): S5-19. https://doi.org/10.1016/j.ijgo.2009.07.016.

Lawn, Joy, Kenji Shibuya, and Claudia Stein. "No Cry at Birth: Global Estimates of Intrapartum Stillbirths and Intrapartum-Related Neonatal Deaths." Bulletin of the World Health Organization 83 (June 2005): 409-17. https://doi.org/10.1590/S0042-96862005000600008.

Lee, Anne CC, Joy E. Lawn, Simon Cousens, Vishwajeet Kumar, David Osrin, Zulfiqar A. Bhutta, Steven N. Wall, Allyala K. Nandakumar, Uzma Syed, and Gary L. Darmstadt. "Linking Families and Facilities for Care at Birth: What Works to Avert Intrapartum-Related Deaths?" International Journal of Gynecology \& Obstetrics, Intrapartum-Related Deaths: Evidence for Action, 107 (October 1, 2009): S65-88. https://doi.org/10.1016/j.ijgo.2009.07.012.

Lee, Soo-Young, and Andrew B. Whitford. "Government Effectiveness in Comparative Perspective." Journal of Comparative Policy Analysis: Research and Practice 11, no. 2 (June 1, 2009): 249-81. https://doi.org/10.1080/13876980902888111.

Lipset, Seymour Martin. "Some Social Requisites of Democracy: Economic Development and Political Legitimacy." American Political Science Review 53, no. 1 (1959): 69-105. 
Little, Daniel. Varieties of Social Explanation: An Introduction to the Philosophy of Social Science. Westview Press, 1991.

Lonsdale, John. "Editorial: Agency in Tight Corners: Narrative and Initiative in African History." Journal of African Cultural Studies 13, no. 1 (2000): 5-16.

Lori, Jody R., and Joyceen S. Boyle. "Cultural Childbirth Practices, Beliefs, and Traditions in Postconflict Liberia." Health Care for Women International 32, no. 6 (2011): 454-473. https://doi.org/10.1080/07399332.2011.555831.

Macinko, James, Maria de Fátima Marinho de Souza, Frederico C. Guanais, and Celso Cardoso da Silva Simões. “Going to Scale with Community-Based Primary Care: An Analysis of the Family Health Program and Infant Mortality in Brazil, 1999-2004." Social Science \& Medicine 65, no. 10 (November 2007): 2070-80. https://doi.org/10.1016/j.socscimed.2007.06.028.

Macro International Incorporated. “Sampling Manual.” Calverton, Maryland, 1996. https://www.google.com/search?safe=off\&client=safari\&rls=en\&ei=4j30XO 7wJuq00gKg6LLAAw\&q=sampling+manual+macro+international+incorpoar ted+1996\&oq=sampling+manual+macro+international+incorpoarted+1996 \&gs_l=psy-ab.3...0i71l2.0.0..4745...0.0..0.0.0.............gws-wiz.7A5bE0jby2U. Magnusson, Bruce A., and John F. Clark. "Understanding Democratic Survival and Democratic Failure in Africa: Insights from Divergent Democratic Experiments in Benin and Congo (Brazzaville)." Comparative Studies in Society and History 47, no. 3 (2005): 552-582. 
Makinen, William Martin. Assessment of the Private Health Sector in Republic of Congo. Investment Climate in Health Series Assessment of the Private Health Sector in the Republic of Congo. Washington, DC: International Bank for Reconstruction and Development/World Bank, 2012.

Manandhar, Dharma S., David Osrin, Bhim Prasad Shrestha, Natasha Mesko, Joanna Morrison, Kirti Man Tumbahangphe, Suresh Tamang, Sushma Thapa, Dej Shrestha, and Bidur Thapa. "Effect of a Participatory Intervention with Women's Groups on Birth Outcomes in Nepal: Cluster-Randomised Controlled Trial." The Lancet 364, no. 9438 (2004): 970-979.

Maslowsky, Julie, Sara Frost, C. Emily Hendrick, Freddy O. Trujillo Cruz, and Sofia D. Merajver. "Effects of Postpartum Mobile Phone-Based Education on Maternal and Infant Health in Ecuador." International Journal of Gynecology \& Obstetrics 134, no. 1 (July 1, 2016): 93-98. https://doi.org/10.1016/j.ijgo.2015.12.008.

Matthew Fuss. "Increasing Health Care Access in Yemen Through Community-Based Health Insurance." Indiana Journal of Global Legal Studies 23, no. 2 (2016): 795-818. https://doi.org/10.2979/indjglolegstu.23.2.0795.

Maxwell, Joan. "The Prevention of Prematurity: A Strategy to Reduce Infant Mortality in the District of Columbia.," 1982.

Mbonye, Anthony K. "Prevalence of Childhood Illnesses and Care-Seeking Practices in Rural Uganda." Research article. The Scientific World Journal, 2003. https://doi.org/10.1100/tsw.2003.52. 
McFerson, Hazel M. “Governance and Hyper-Corruption in Resource-Rich African Countries." Third World Quarterly 30, no. 8 (2009): 1529-1547.

McGillivray, Mark, Simon Feeny, Niels Hermes, and Robert Lensink. "Controversies over the Impact of Development Aid: It Works; It Doesn't; It Can, but That Depends...." Journal of International Development: The Journal of the Development Studies Association 18, no. 7 (2006): 1031-1050.

McKenzie, L, and M Ellis. “Community-Based Interventions to Improve Neonatal Survival in Low-Resource Settings." Annals Of Tropical Paediatrics 31, no. 3 (2011): 191-99. https://doi.org/10.1179/1465328111Y.0000000002. Melese, Ayelech Tiruwha, and A. H. J. (Bert) Helmsing. “Endogenisation or Enclave Formation? The Development of the Ethiopian Cut Flower Industry*." The Journal of Modern African Studies 48, no. 1 (March 2010): 35-66. https://doi.org/10.1017/S0022278X09990218.

Merali, Hasan S, Stuart Lipsitz, Nathanael Hevelone, Atul A Gawande, Angela Lashoher, Priya Agrawal, and Jonathan Spector. "Audit-Identified Avoidable Factors in Maternal and Perinatal Deaths in Low Resource Settings: A Systematic Review." BMC Pregnancy and Childbirth 14, no. 1 (December 2014): 280. https://doi.org/10.1186/1471-2393-14-280.

Mikounou Louya, Vivaldie, Christevy Vouvoungui, Félix Koukouikila-Koussounda, Francisco Veas, Simon Charle Kobawila, and Francine Ntoumi. "Molecular Characterization of Norovirus Infection Responsible for Acute Diarrhea in Congolese Hospitalized Children under Five Years Old in Brazzaville, 
Republic of Congo." International Journal of Infectious Diseases 88 (November 1, 2019): 41-48. https://doi.org/10.1016/j.ijid.2019.07.034.

Ministry of Public Health- Yemen. "Health Sector Reform in the Republic of Yemen: Strategy for Reform," October 2000. http://www.mophpye.org/docs/HSR_Strategy.pdf.

Moore, Jina. "In Republic of Congo, a Revolution in Maternal Health." Al Jazeera America, 2013. https://www.google.com/search?client=safari\&rls=en\&q=in+republic + of + co ngo,+a+revolution+in+maternal+health\&ie=UTF-8\&oe=UTF-8.

MOPHP/Yemen, Central Statistical Organizaton (CSO), Pan Arab Program for Family Health (PAPFAM), and ICF International. "Yemen National Health and Demographic Survey 2013." Rockville, Maryland, USA, 2015.

Morel, E. D. Edmund Dene. The British Case in French Congo; the Story of a Great Injustice, Its Causes and Its Lessons. New York: Negro Universities Press, 1969. Mosley, Paul. "Aid-Effectiveness: The Micro-Macro Paradox." Ids Bulletin 17, no. 2 (1986): 22-27.

- - Overseas Aid: Its Defence and Reform. Wheatsheaf Books, 1987.

Mosley, W. Henry, and Lincoln C. Chen. "An Analytical Framework for the Study of Child Survival in Developing Countries." Population and Development Review 10 (1984): 25. https://doi.org/10.2307/2807954.

Moss, William, Gary L Darmstadt, David R Marsh, Robert E Black, and Mathuram Santosham. "Research Priorities for the Reduction of Perinatal and Neonatal 
Morbidity and Mortality in Developing Country Communities." Journal of Perinatology 22, no. 6 (September 2002): 484-95.

https://doi.org/10.1038/sj.jp.7210743.

Moulemvo, André. "Impact of Capital Flight on Public Social Expenditure in CongoBrazzaville." African Development Review 28, no. S1 (2016): 113-123.

Moyer, Cheryl A., Lia Tadesse, and Senait Fisseha. "The Relationship between Facility Delivery and Infant Immunization in Ethiopia." International Journal of Gynecology \& Obstetrics 123, no. 3 (December 1, 2013): 217-20. https://doi.org/10.1016/j.ijgo.2013.06.030.

Muller, Edward N. "Democracy, Economic Development, and Income Inequality.” American Sociological Review, 1988, 50-68.

Nair, Nirmala, Prasanta Tripathy, Anthony Costello, and Audrey Prost. "Mobilizing Women's Groups for Improved Maternal and Newborn Health: Evidence for Impact, and Challenges for Sustainability and Scale Up." International Journal of Gynecology \& Obstetrics, World Report on Women's Health 2012:, 119 (October 1, 2012): S22-25. https://doi.org/10.1016/j.ijgo.2012.03.014. Nair, Nirmala, Prasanta Tripathy, Audrey Prost, Anthony Costello, and David Osrin. “Improving Newborn Survival in Low-Income Countries: Community-Based Approaches and Lessons from South Asia." PLoS Medicine 7, no. 4 (2010): e1000246.

Nalwadda, Christine Kayemba, Peter Waiswa, Juliet Kiguli, Gertrude Namazzi, Sarah Namutamba, Göran Tomson, Stefan Peterson, and David Guwatudde. “High 
Compliance with Newborn Community-to-Facility Referral in Eastern Uganda: An Opportunity to Improve Newborn Survival." PLOS ONE 8, no. 11 (November 29, 2013): e81610. https://doi.org/10.1371/journal.pone.0081610.

UNICEF. "New Data Show Child Mortality Rates Falling Faster than Ever." Accessed June 5, 2019. https://www.unicef.org/media/media_75892.html.

"Newborns: Reducing Mortality." Accessed March 25, 2019.

https://www.who.int/news-room/fact-sheets/detail/newborns-reducingmortality.

Nishishiba, Masami. Research Methods and Statistics for Public and Nonprofit Administrators: A Practical Guide. SAGE Research Methods Books \& Reference. Los Angeles: SAGE, 2014.

Organization, World Health. "Neonatal and Perinatal Mortality: Country, Regional and Global Estimates," 2006.

Organization/Yemen, Central Statistical, and Macro International. "Yemen Demographic and Maternal and Child Health Survey 1997," 1998. http://dhsprogram.com/publications/publication-FR94-DHS-FinalReports.cfm.

Øvretveit, John, and Abdul Al Serouri. "Hospital Quality Management System in a Low Income Arabic Country: An Evaluation." International Journal of Health Care Quality Assurance 19, no. 6 (2006): 516-532. https://doi.org/10.1108/09526860610686999. 
Perry, Henry B., David S. Shanklin, and Dirk G. Schroeder. "Impact of a CommunityBased Comprehensive Primary Healthcare Programme on Infant and Child Mortality in Bolivia." Journal of Health, Population and Nutrition 21, no. 4 (December 2003): 383-95.

Persson, Lars Åke, Anisur Rahman, Rodolfo Peña, Wilton Perez, Aimable Musafili, and Dinh Phuong Hoa. "Child Survival Revolutions Revisited - Lessons Learned from Bangladesh, Nicaragua, Rwanda and Vietnam." Acta Paediatrica 106, no. 6 (June 2017): 871-77. https://doi.org/10.1111/apa.13830.

Posner, Daniel N., and Daniel J. Young. “Term Limits: Leadership, Political Competition and the Transfer of Power." Institutions and Democracy in Africa: How the Rules of the Game Shape Political Developments, 2018, 260-278.

Pouw, Nicky R. M., and Ad de Bruijne. "Strategic Governance for Inclusive Development." The European Journal of Development Research 27, no. 4 (September 1, 2015): 481-87. https://doi.org/10.1057/ejdr.2015.53.

Pritchett, Lant, and Lawrence H. Summers. "Wealthier Is Healthier.” The Journal of Human Resources 31, no. 4 (1996): 841-68. https://doi.org/10.2307/146149.

Rajaratnam, Julie Knoll, Jake R. Marcus, Abraham D. Flaxman, Haidong Wang, Alison Levin-Rector, Laura Dwyer, Megan Costa, Alan D. Lopez, and Christopher JL Murray. "Neonatal, Postneonatal, Childhood, and under-5 Mortality for 187 Countries, 1970-2010: A Systematic Analysis of Progress towards 
Millennium Development Goal 4." The Lancet 375, no. 9730 (2010): 19882008.

Rajkumar, Andrew Sunil, and Vinaya Swaroop. "Public Spending and Outcomes: Does Governance Matter?" Journal of Development Economics 86, no. 1 (2008): 96-111.

Reij, C. P., and E. M. A. Smaling. "Analyzing Successes in Agriculture and Land Management in Sub-Saharan Africa: Is Macro-Level Gloom Obscuring Positive Micro-Level Change?" Land Use Policy 25, no. 3 (2008): 410-420. https://doi.org/10.1016/j.landusepol.2007.10.001.

IMF. "Republic of Congo : Selected Issues." Accessed October 29, 2019. https://www.imf.org/en/Publications/CR/Issues/2016/12/31/Republic-ofCongo-Selected-Issues-41889.

Riaz, Saadia, and Louise Condon. "The Experiences of Breastfeeding Mothers Returning to Work as Hospital Nurses in Pakistan: A Qualitative Study." Women and Birth, 2018.

Ridpath, Alison Delano, Heather Melissa Scobie, Messeret Eshetu Shibeshi, Ahmadu Yakubu, Flint Zulu, Azhar Abid Raza, Balcha Masresha, and Rania Tohme. "Progress towards Achieving and Maintaining Maternal and Neonatal Tetanus Elimination in the African Region." The Pan African Medical Journal 27, no. Suppl 3 (2017): 24. https://doi.org/10.11604/pamj.supp.2017.27.3.11783. 
Rosato, Mikey, Glenn Laverack, Lisa Howard Grabman, Prasanta Tripathy, Nirmala Nair, Charles Mwansambo, Kishwar Azad, et al. “Community Participation: Lessons for Maternal, Newborn, and Child Health." The Lancet 372, no. 9642 (September 2008): 962-71. https://doi.org/10.1016/S01406736(08)61406-3.

Ross, Michael. "Is Democracy Good for the Poor?” American Journal of Political Science 50, no. 4 (October 2006): 860-74.

Saadeh, Randa Jarudi. “The Baby-Friendly Hospital Initiative 20 Years On: Facts, Progress, and the Way Forward." Journal of Human Lactation 28, no. 3 (August 1, 2012): 272-75. https://doi.org/10.1177/0890334412446690.

Saha, Subir, and M. Kabir. "Survival of Newborns: Implications for Achieving the Millennium Development Goal in Bangladesh." World Health \& Population 11, no. 1 (2009): 5-13. https://doi.org/10.12927/whp.2009.20990.

Saleh, Shadi S., Mohamad S. Alameddine, Nabil M. Natafgi, Awad Mataria, Belgacem Sabri, Jamal Nasher, Moez Zeiton, Shaimaa Ahmad, and Sameen Siddiqi. "The Path towards Universal Health Coverage in the Arab Uprising Countries Tunisia, Egypt, Libya, and Yemen." The Lancet 383, no. 9914 (2014): 368381. https://doi.org/10.1016/S0140-6736(13)62339-9.

Sartorius, Benn KD, and Kurt Sartorius. “Global Infant Mortality Trends and Attributable Determinants-an Ecological Study Using Data from 192 Countries for the Period 1990-2011." Population Health Metrics 12, no. 1 (2014): 29 . 
Schell, Carl Otto, Marie Reilly, Hans Rosling, Stefan Peterson, and Anna Mia Ekström.

"Socioeconomic Determinants of Infant Mortality: A Worldwide Study of 152 Low-, Middle-, and High-Income Countries." Scandinavian Journal of Public

Health 35, no. 3 (May 2007): 288-97.

https://doi.org/10.1080/14034940600979171.

Schuppert, Gunnar Folke. "Partnerships.” The SAGE Handbook of Governance.

University of California, Berkeley: SAGE, 2011.

Security Council. "Letter Dated 20 February 2015 from the Panel of Experts on

Yemen Established Pursuant to Security Council Resolution 2140 (2014)

Addressed to the President of the Security Council," February 20, 2015.

Sen, Amartya. Development as Freedom. 1st ed. New York: Knopf, 1999.

Sen, Kunal. "Governance and Development Outcomes in Asia." Working Paper. ADB

Economics Working Paper Series, No. 384. Manila: Asian Development Bank (ADB), 2014.

Sharma, Shalendra. "Democracy, Good Governance, and Economic Development." Taiwan Journal of Democracy 3, no. 1 (July 2007): 29-62.

Shepsle, Kenneth A. "Rational Choice Institutionalism." The Oxford Handbook of Political Institutions, 2006, 23-38.

Sirili, Nathanael, Gasto Frumence, Angwara Kiwara, Mughwira Mwangu, Isabel Goicolea, and Anna-Karin Hurtig. "Public Private Partnership in the Training of Doctors after the 1990s' Health Sector Reforms: The Case of Tanzania." Human Resources for Health 17, no. 1 (2019): 33. 
Soeters, Robert, Peter Bob Peerenboom, Pacifique Mushagalusa, and Célestin Kimanuka. "Performance-Based Financing Experiment Improved Health Care in the Democratic Republic of Congo." Health Affairs (Project Hope) 30, no. 8 (2011): 1518-27. https://doi.org/10.1377/hlthaff.2009.0019.

Tchana Tchana, Fulbert, and Etaki Wa Dzon. "Republic of the Congo - Report Monitoring the Economic and Financial Situation." The World Bank, December 4, 2015. http://documents.worldbank.org/curated/en/211851467992000410/Repu blic-of-the-Congo-Report-monitoring-the-economic-and-financial-situation.

Tchibindat, Félicité, Yves Martin-Prevel, Patrick Kolsteren, Bernard Maire, and Francis Delpeuch. "Bringing Together Viewpoints of Mothers and Health Workers to Enhance Monitoring and Promotion of Growth and Development of Children: A Case Study from the Republic of Congo." Journal of Health, Population and Nutrition 22, no. 1 (2004): 59-67.

Teune, Henry, and Adam Przeworski. The Logic of Comparative Social Inquiry. New York: Wiley-Interscience, 1970.

“The DHS Program - Demographic and Health Survey (DHS)." Accessed June 2, 2019. https://dhsprogram.com/What-We-Do/Survey-Types/DHS.cfm.

The World Bank. "GDP Growth (Annual \%).” World Bank national accounts data, and OECD National Accounts data files, 2017. https://data.worldbank.org/indicator/NY.GDP.MKTP.KD.ZG?view=chart. 
The World Bank, The World. "Congo - Health Sector Services Development Project." The World Bank, March 12, 2008. http://documents.worldbank.org/curated/en/400941468247240552/Cong o-Health-Sector-Services-Development-Project.

Tripathy, Prasanta, Nirmala Nair, Sarah Barnett, Rajendra Mahapatra, Josephine Borghi, Shibanand Rath, Suchitra Rath, Rajkumar Gope, Dipnath Mahto, and Rajesh Sinha. “Effect of a Participatory Intervention with Women's Groups on Birth Outcomes and Maternal Depression in Jharkhand and Orissa, India: A Cluster-Randomised Controlled Trial." The Lancet 375, no. 9721 (2010): 1182-1192.

Troutman, Adewale. "Addressing U.S. Infant Mortality through Healthy Start Programs." Nation's Health 43, no. 2 (March 2013): 3-3.

UNDP. "Human Development Indices and Indicators: 2018 Statistical Update | Human Development Reports," 2018. http://hdr.undp.org/en/content/human-development-indices-indicators2018-statistical-update.

UNFPA. “Momentum Building for Maternal Health in Republic of Congo." UNFPA News, 2010. https://www.google.com/search?safe=off\&client=safari\&rls=en\&ei=y5vMXI X1F97B0PEP0KaOqAs\&q=momentum+building+for+maternal+health+in+re public+of+congo\&oq=momentum+building+for+maternal+helth+in+republic +of+congo\&gs_l=psy- 
ab.1.0.35i304i39.15861.16517..18705 ...0.0..0.75.138.2.............gws-

wiz.1LsnqeVOMEM.

_—_. "New Push to Promote Women's Health in Africa," 2006. /press/new-pushpromote-womens-health-africa.

UNICEF. “UNICEF Humanitarian Action Report 2008 Republic of the Congo Brazzaville." Accessed April 11, 2019. https://www.google.com/search?client=safari\&rls=en\&q=unicef+humanitari an+action+report+2008+republic + of + the + congo + brazzaville\&ie=UTF$8 \&$ oe $=$ UTF- 8 .

United Nations. "Innovations Driving Health MDGS in Eritrea." Health Millennium Development Goals Report Abridged Version. United Nations, September 2014.

USAID. “Congo (Brazzaville), Journey to Self-Reliance: FY 2020 Country Roadmap," n.d. https://selfreliance.usaid.gov/country/congo-brazzaville.

———. "MCHIP End of Project Report," 2014.

———. "Yemen, Journey to Self-Reliance: FY 2020 Country Roadmap," n.d. https://selfreliance.usaid.gov/country/yemen.

Van Lerberghe, Wim, Zoe Matthews, Endang Achadi, Chiara Ancona, James Campbell, Amos Channon, Luc De Bernis, Vincent De Brouwere, Vincent Fauveau, and Helga Fogstad. "Country Experience with Strengthening of Health Systems and Deployment of Midwives in Countries with High Maternal Mortality." The Lancet 384, no. 9949 (2014): 1215-1225. 
Vandelaer, J. “Tetanus in Developing Countries: An Update on the Maternal and Neonatal Tetanus Elimination Initiative." Vaccine 21, no. 24 (July 28, 2003): 3442-45. https://doi.org/10.1016/S0264-410X(03)00347-5.

Vansina, Jan. Paths in the Rainforests: Toward a History of Political Tradition in Equatorial Africa. Madison, Wis.: University of Wisconsin Press, 1990.

Victora, Cesar Gomes, and Fernando Celso Barros. "Infant Mortality Due to Perinatal Causes in Brazil: Trends, Regional Patterns and Possible Interventions." São Paulo Medical Journal 119, no. 1 (2001): 33-42.

Violari, Avy, Mark F. Cotton, Diana M. Gibb, Abdel G. Babiker, Jan Steyn, Shabir A. Madhi, Patrick Jean-Philippe, and James A. McIntyre. “Early Antiretroviral Therapy and Mortality among HIV-Infected Infants." New England Journal of Medicine 359, no. 21 (2008): 2233-2244.

Wang, Haidong, Zulfiqar A Bhutta, Matthew M Coates, Megan Coggeshall, Lalit Dandona, Khassoum Diallo, Elisabeth Barboza Franca, et al. “Global, Regional, National, and Selected Subnational Levels of Stillbirths, Neonatal, Infant, and under-5 Mortality, 1980-2015: A Systematic Analysis for the Global Burden of Disease Study 2015." The Lancet 388, no. 10053 (October 2016): 1725-74. https://doi.org/10.1016/S0140-6736(16)31575-6.

WHO. “National Health Strategy for Yemen 2011-2025.” WHO EMRO: Health systems development programmes, Yemen. Accessed April 24, 2019. http://www.emro.who.int/yem/programmes/health-systemsdevelopment.html. 
Williams, Walter. Social Policy Research and Analysis: The Experience in the Federal Social Agencies. American Elsevier Publishing Company, 1971.

Wise, Paul H., and Gary L. Darmstadt. "Strategic Governance: Addressing Neonatal Mortality in Situations of Political Instability and Weak Governance."

Seminars in Perinatology, Global Perinatal Medicine, 39, no. 5 (August 2015): 387-92. https://doi.org/10.1053/j.semperi.2015.06.008.

World Bank. "Mortality Rate, Infant (per 1,000 Live Births) | Data." Accessed April 8, 2019. https://data.worldbank.org/indicator/SP.DYN.IMRT.IN.

_——. "Performance-Based Financing for Health in the Republic of Congo: Impact Evaluation Concept Note," 2014. http://microdata.worldbank.org/index.php/catalog/2844/download/40107

———. "Republic of Congo: Health System Strengthening Project II (PDSS II)," November 25, 2013. http://documents.worldbank.org/curated/en/236521468025764060/pdf/8 10090PAD0P143010Box379877B000U0090.pdf.

_—_. "WB to Help Republic of the Congo Provide Better Healthcare for Nearly 1.5 Million Mothers, Children." Text/HTML. World Bank, December 20, 2013. https://www.worldbank.org/en/news/press-release/2013/12/20/wbcongo-healthcare-mothers-children. 
World Health Organization. "Report of a WHO Technical Consultation on Birth Spacing: Geneva, Switzerland 13-15 June 2005." Geneva: World Health Organization, 2007.

Yemen Ministry of Public Health. "Leading Yemen to Better Health Safety and Well Being." Ministry of Health, August 17, 2015. http://www.moh.gov.ye/english/message.html.

Yin, Robert K. Case Study Research: Design and Methods, 3rd Edition. 3rd edition. Thousand Oaks, Calif: SAGE Publications, Inc, 2002.

Zeng, Wu, Donald S. Shepard, Jean de Dieu Rusatira, Aaron P. Blaakman, and Bernice M. Nsitou. "Evaluation of Results-Based Financing in the Republic of the Congo: A Comparison Group Pre-Post Study." Health Policy and Planning 33, no. 3 (April 1, 2018): 392-400. https://doi.org/10.1093/heapol/czx195.

Zweifel, Thomas D., and Patricio Navia. “Democracy, Dictatorship, and Infant Mortality." Journal of Democracy 11, no. 2 (2000): 99-114. 
Appendix A: Decrease in IMR from 1996 to 2014

The following scatterplot shows the decrease in infant mortality rate per 1,000 live births from 1996 to 2014 in 106 developing countries.

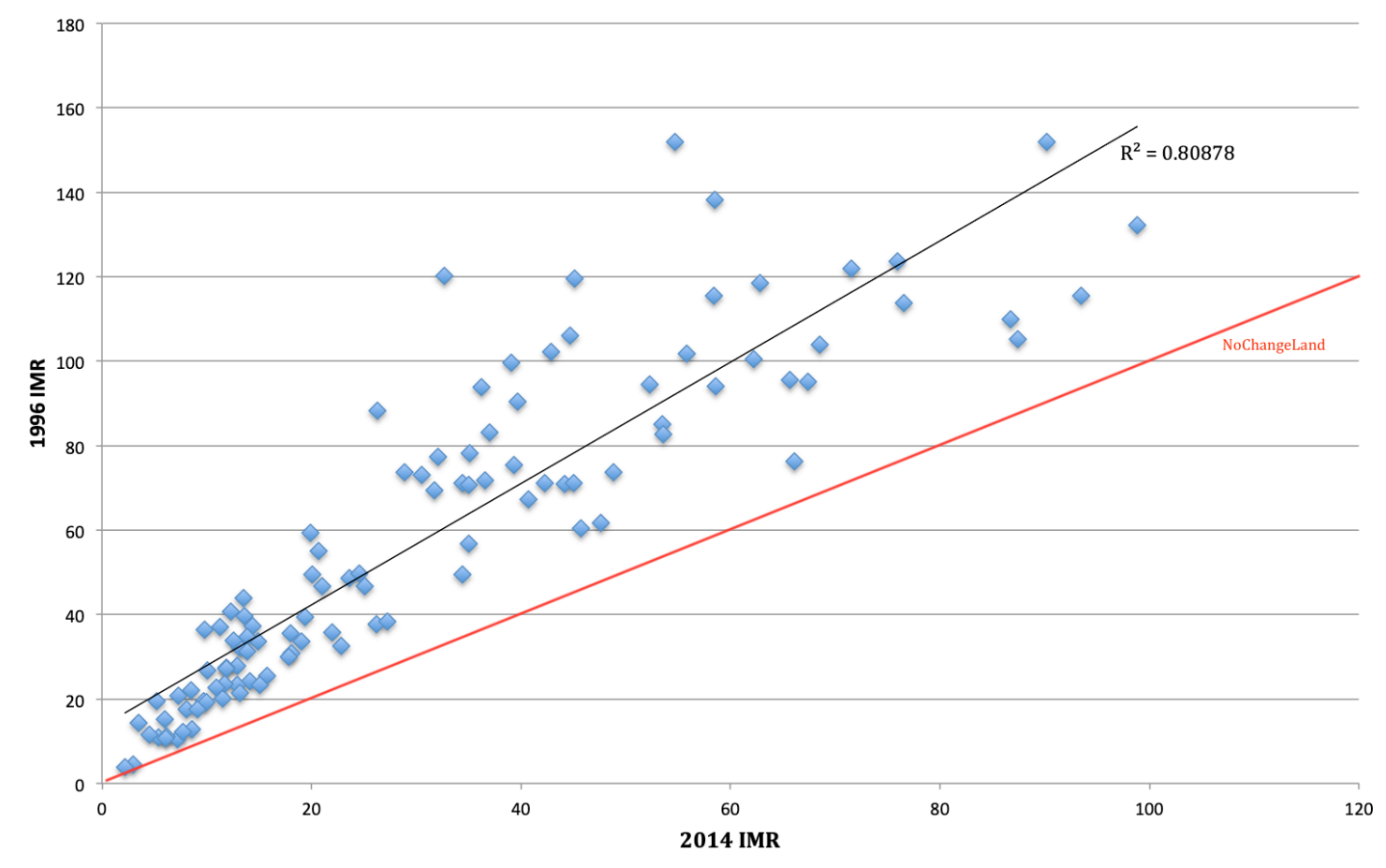

1996 and 2014 IMR for 106 developing countries

The average IMR in 1996 for 106 developing countries was 58.42 per 1,000 live births. In 2014, the average IMR for the same countries was 31.21 per 1,000 live births. The average percent decline in IMR for the 106 countries is $48 \% 570$.

570 World Bank data, author's calculation. 
Appendix B: Republic of Congo Data

Unfortunately there is not earlier data from either the DHS or MICS from the late 1990s. Longitudinal data demonstrates where some change occurred and reveals some there is improvement at a micro level (individual and community) even as the macro level (national) remains challenged. Research and news articles are also examined to reveal how the IMR improved over a specific timeframe. While there are improvements in the Republic of Congo overall, it is important to note that significant differences exist between rural and urban communities in earlier and more current datasets.

The Demographic Health Surveys from 2005 and 2011-2012 do not ask identical sets of questions; however, there are some similar questions and this case study will focus on some of those select data points to see how they potentially impact the IMR over the six year period. The duplicate areas covered in both DHS studies are: housing characteristics, household possessions, family planning messages, literacy, maternal mortality, exposure to media, birth intervals, age of first birth, knowledge of contraception, infant mortality, number of ANC visits, women who had tetanus toxoid injections during their last pregnancy, number of women receiving ANC from trained personnel, of women who received antenatal care were they informed of signs of pregnancy complications and had routine checks, place of delivery, assistance at delivery, vaccinations before 12 months of age, knowledge of oral rehydration salts (ORS), use of mosquito nets and knowledge of specific types of HIV transmission. 
Appendix C: Select Survey Data for Republic of Congo

Republic of Congo: Select comparison of survey data from Demographic Health

Surveys (DHS) 2005, and (DHS) 2011-2012, and the Multiple Indicator Cluster Survey (MICS) 2014-2015.

\begin{tabular}{|c|c|c|c|c|c|c|c|}
\hline & DHS (2005) & $\begin{array}{c}\text { DHS } \\
\text { Urban } \\
\text { (2005) }\end{array}$ & $\begin{array}{c}\text { DHS } \\
\text { Rural } \\
\text { (2005) }\end{array}$ & $\begin{array}{c}\text { DHS } \\
\text { (2011- } \\
\text { 2012) }\end{array}$ & $\begin{array}{l}\text { DHS } \\
\text { Urban } \\
\text { (2011- } \\
\text { 2012) }\end{array}$ & $\begin{array}{c}\text { DHS } \\
\text { Rural } \\
\text { (2011- } \\
\text { 2012) }\end{array}$ & $\begin{array}{c}\text { MICS } \\
(2014 \\
- \\
2015)\end{array}$ \\
\hline $\begin{array}{l}\text { Housing } \\
\text { characteristics }\end{array}$ & & & & & & & \\
\hline $\begin{array}{l}\text { Drinking water } \\
\text { piped into } \\
\text { residence/Fau } \\
\text { cet in housing }\end{array}$ & 2.6 & 4.6 & 0.5 & 3.5 & 5.3 & 0.4 & \\
\hline $\begin{array}{l}\text { Unprotected } \\
\text { source }\end{array}$ & 13.7 & 0.9 & 27.9 & 8.7 & 1 & 21.9 & \\
\hline Electricity & & & & & & & \\
\hline Yes & 33.8 & 50.8 & 14.8 & 41.6 & 58.9 & 11.7 & \\
\hline No & 66.2 & 49.2 & 85.1 & 58.4 & 41 & 88.2 & \\
\hline $\begin{array}{l}\text { Floor covering } \\
\text { material }\end{array}$ & & & & & & & \\
\hline Earth, sand & 42 & 9.5 & 78.1 & 32.9 & 7.7 & 76.4 & \\
\hline Wood boards & 1.4 & 0.1 & 2.9 & 0.3 & 0.1 & 0.7 & \\
\hline Vinyl strips & 5.1 & 9.4 & 0.3 & 5.4 & 7.8 & 1.2 & \\
\hline Tiles & 3.9 & 7.2 & 0.2 & 6.1 & 9 & 1 & \\
\hline
\end{tabular}




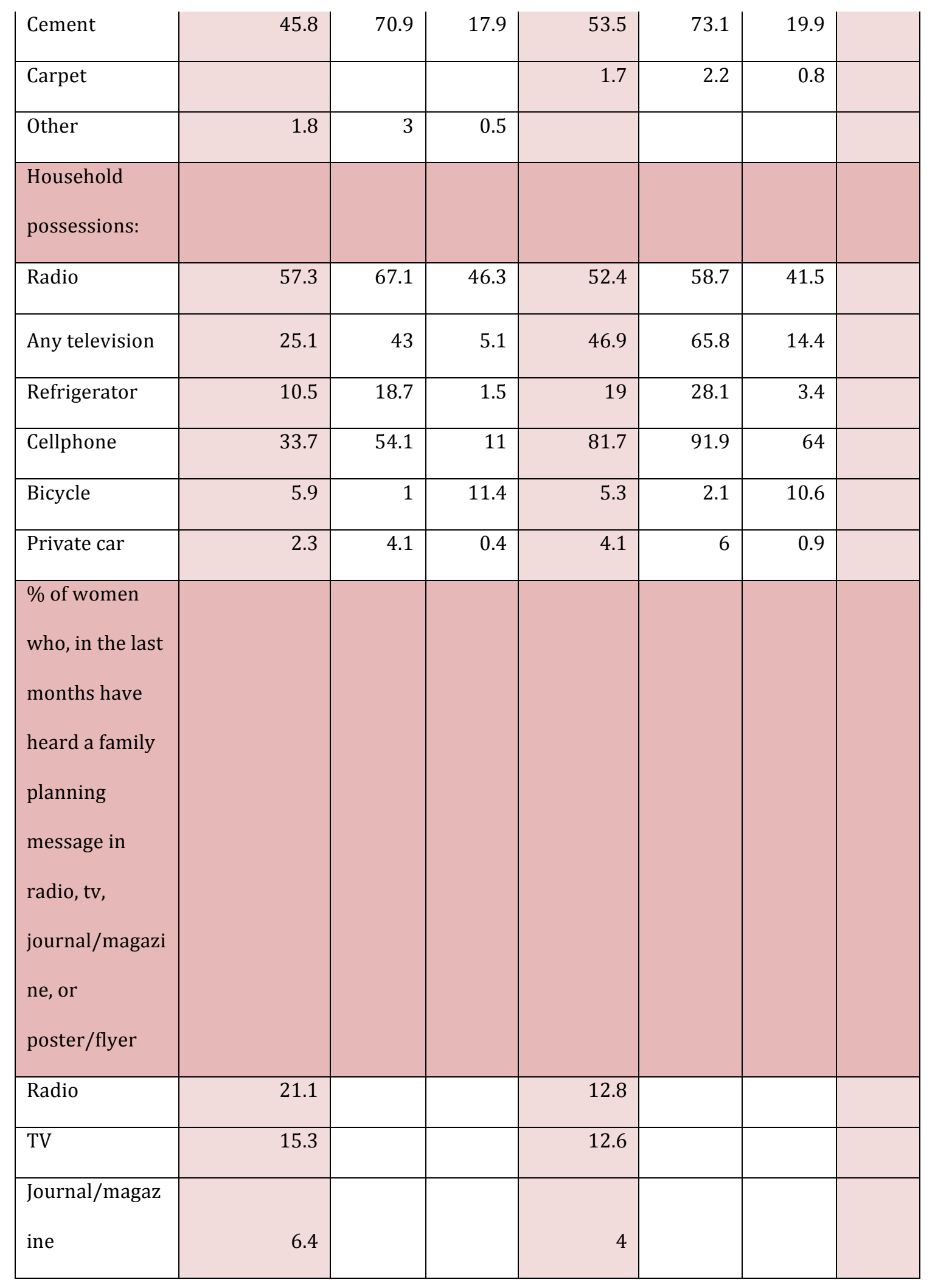




\begin{tabular}{|c|c|c|c|c|c|c|}
\hline$\%$ Literate & 78 & 89 & 63.7 & 82.2 & 90.2 & 64.7 \\
\hline $\begin{array}{l}\text { Maternal } \\
\text { mortality }\end{array}$ & 781 & & & 426 & & \\
\hline $\begin{array}{l}\text { WOMEN } \\
\text { exposed to } \\
\text { media }\end{array}$ & & & & & & \\
\hline $\begin{array}{l}\text { Read } \\
\text { newspaper at } \\
\text { least once } \\
\text { weekly }\end{array}$ & 16.4 & 20.9 & 10.5 & 18.6 & 22.7 & 9.6 \\
\hline $\begin{array}{l}\text { Watch Tv at } \\
\text { least once } \\
\text { weekly }\end{array}$ & 38.1 & 58.8 & 11.1 & 52.6 & 67.1 & 20.9 \\
\hline $\begin{array}{l}\text { Listen to radio } \\
\text { at least once } \\
\text { weekly }\end{array}$ & 45 & 52 & 36 & 35.1 & 38.5 & 27.6 \\
\hline $\begin{array}{l}\text { Exposure to all } \\
\text { three media }\end{array}$ & 7.4 & 11.6 & 1.9 & 9 & 11.7 & 3.1 \\
\hline No media & 37 & 21.3 & 57.4 & 34.8 & 23.2 & 60 \\
\hline Birth intervals: & & & & & & \\
\hline $\begin{array}{l}7 \text { months - } 17 \\
\text { months }\end{array}$ & 5 & 4.1 & 5.7 & 5 & 4.9 & 5.2 \\
\hline $18-23$ & 8.2 & 8 & 8.3 & 9.5 & 9 & 10.2 \\
\hline $24-35$ & 28.6 & 24.5 & 31.8 & 28.8 & 25.6 & 33.1 \\
\hline $36-47$ & 22.7 & 21.4 & 23.6 & 21.9 & 20.9 & 23.1 \\
\hline
\end{tabular}




\begin{tabular}{|c|c|c|c|c|c|c|}
\hline $48+$ & 35.5 & 42 & 30.6 & 34.9 & 39.5 & 28.3 \\
\hline $\begin{array}{l}\text { Age at first } \\
\text { birth: }\end{array}$ & & & & & & \\
\hline $\begin{array}{l}\text { Median age at } \\
\text { first birth for } \\
\text { women age } 25 \text { - } \\
49\end{array}$ & & 19.8 & 19 & & 19.9 & 19 \\
\hline $\begin{array}{l}\text { WOMEN } \\
\text { Knowledge of } \\
\text { contraceptive }\end{array}$ & & & & & & \\
\hline Any method & 97.5 & & & 98.6 & & \\
\hline $\begin{array}{l}\text { Modern } \\
\text { method }\end{array}$ & 95.2 & & & 97.8 & & \\
\hline $\begin{array}{l}\text { Traditional } \\
\text { method }\end{array}$ & 94.8 & & & 95.8 & & \\
\hline $\begin{array}{l}\text { Infant and } \\
\text { child mortality } \\
\text { levels: }\end{array}$ & & & & & & \\
\hline $\begin{array}{l}\text { Neonatal } \\
\text { mortality }\end{array}$ & & 36 & 35 & & 26 & 21 \\
\hline $\begin{array}{l}\text { Post neonatal } \\
\text { mortality }\end{array}$ & & 31 & 58 & & 18 & 29 \\
\hline $\begin{array}{l}\text { Number of } \\
\text { ANC visits: }\end{array}$ & & & & & & \\
\hline 0 & 11.6 & 5.2 & 17.8 & 6.9 & 3.8 & 12.1 \\
\hline
\end{tabular}




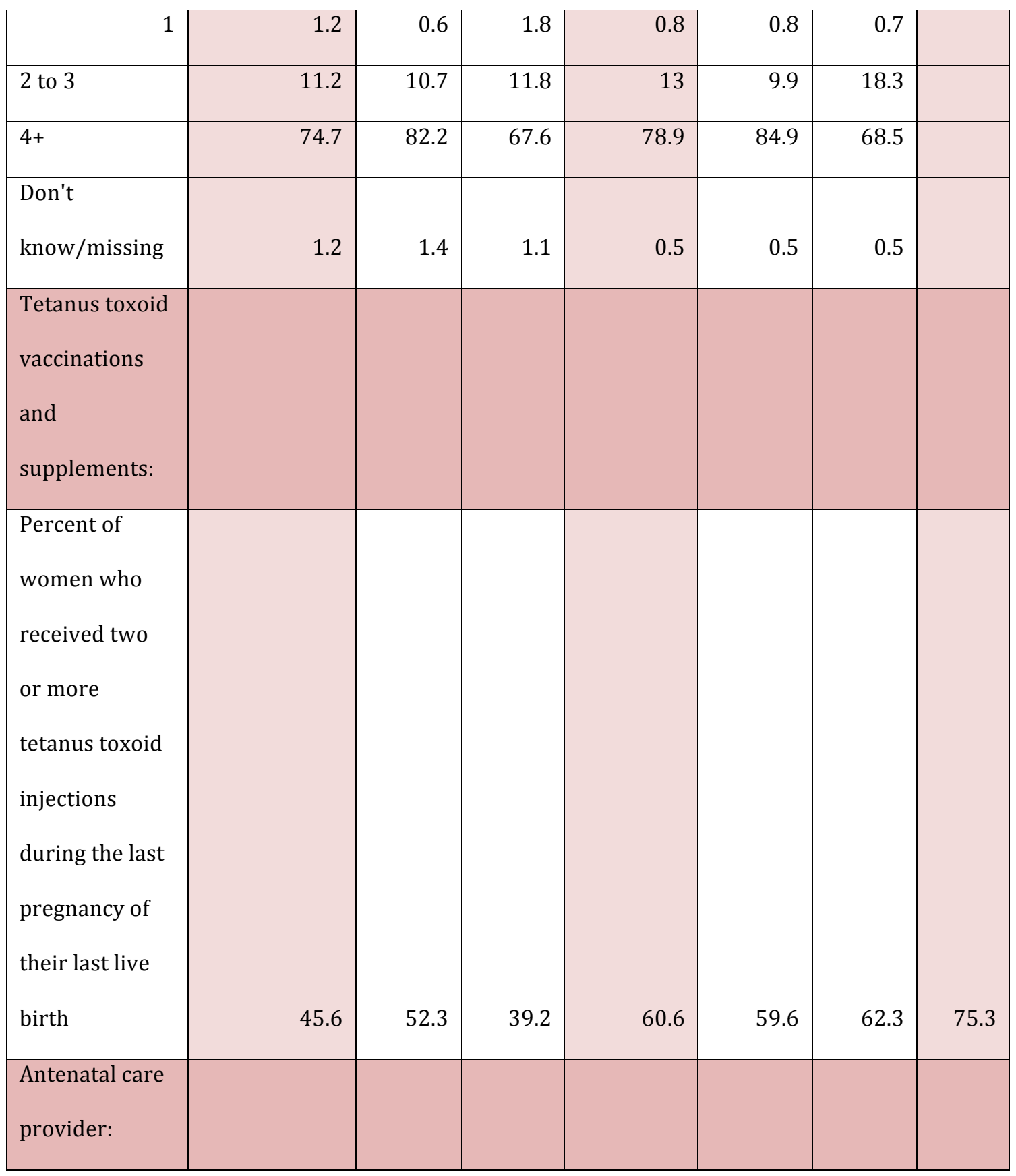




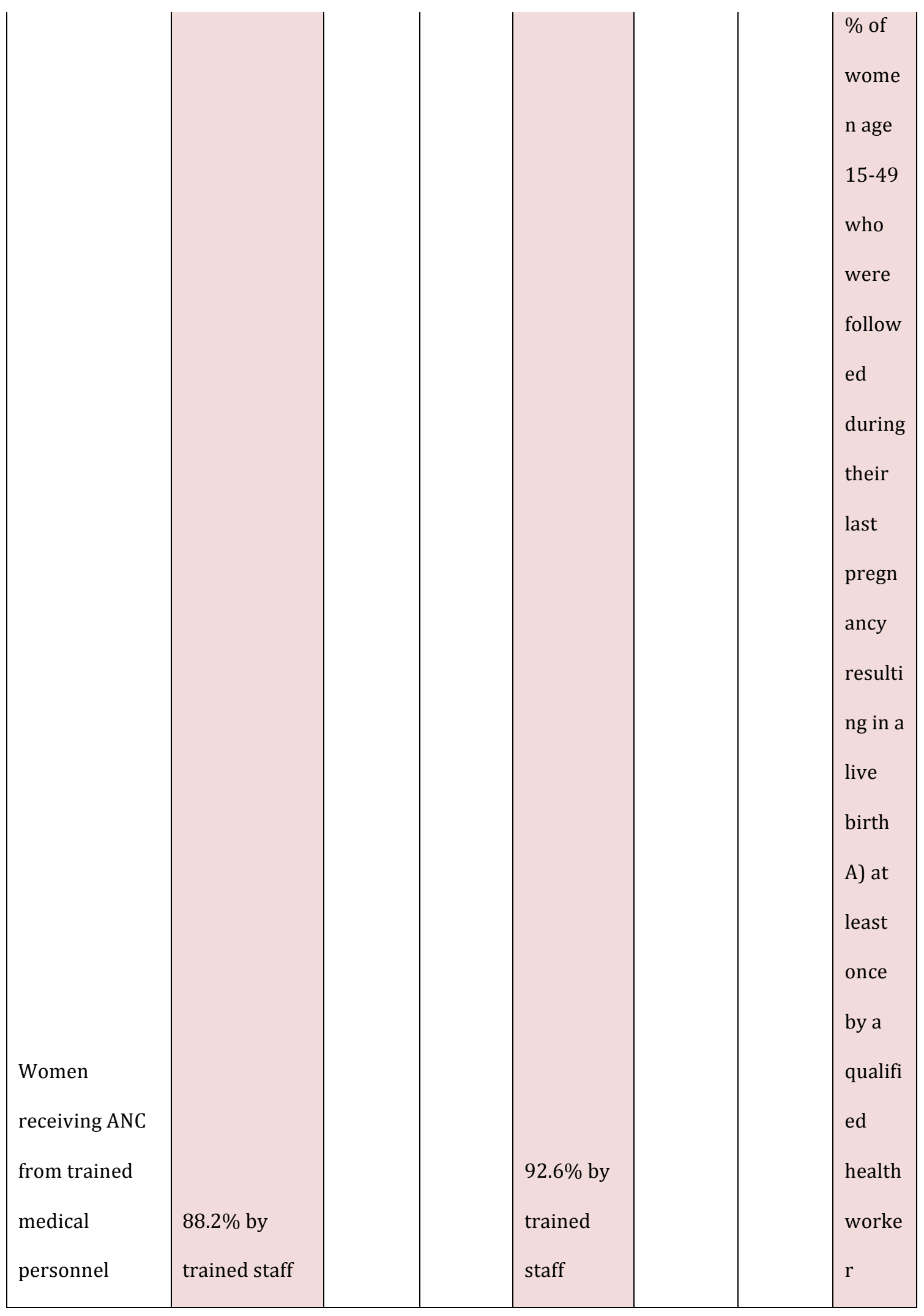




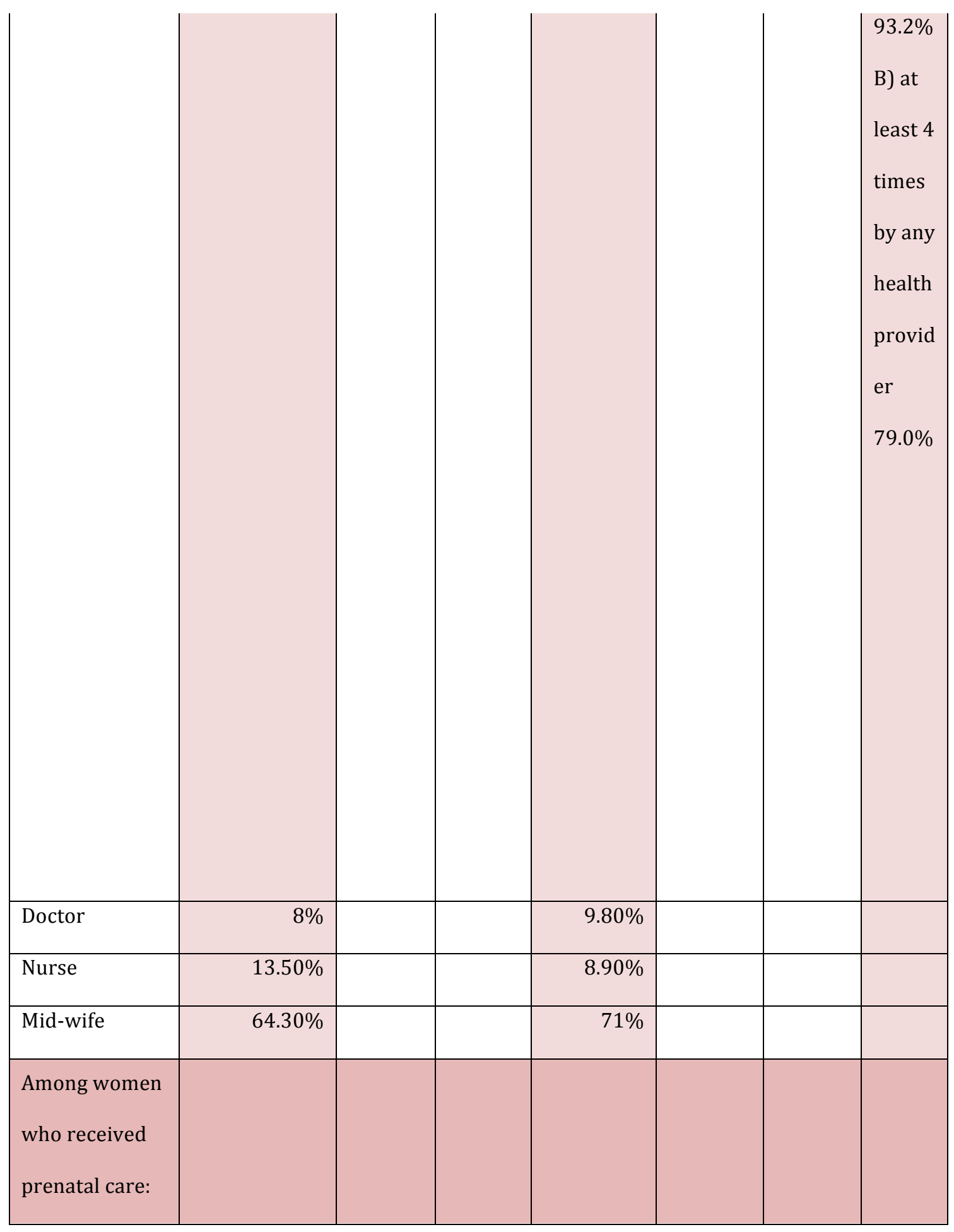




\begin{tabular}{|c|c|c|c|c|c|c|}
\hline $\begin{array}{l}\text { Informed of } \\
\text { signs of } \\
\text { pregnancy } \\
\text { complications }\end{array}$ & $40.20 \%$ & 41.5 & 38.7 & $62 \%$ & 61.2 & 64.3 \\
\hline Weight & & & & & & \\
\hline measured & $97.70 \%$ & 99.1 & 96.2 & $99 \%$ & 99.7 & 97.3 \\
\hline Size measured & $93.60 \%$ & 97.2 & 89.6 & $96 \%$ & 97.8 & 91.7 \\
\hline Blood pressure & $94.40 \%$ & 98.2 & 90.2 & $98 \%$ & 99.1 & 95.4 \\
\hline Urine sample & $94.60 \%$ & 98.9 & 89.8 & $95 \%$ & 99.2 & 86.7 \\
\hline Blood sample & $93.20 \%$ & 97.6 & 88.3 & $94 \%$ & 98.9 & 84.8 \\
\hline $\begin{array}{l}\text { \% received } \\
\text { iron tablets or } \\
\text { syrups }\end{array}$ & $52.80 \%$ & 67.7 & 38.5 & $84 \%$ & 89.7 & 74.5 \\
\hline $\begin{array}{l}\text { Place of } \\
\text { delivery: }\end{array}$ & & & & & & \\
\hline
\end{tabular}




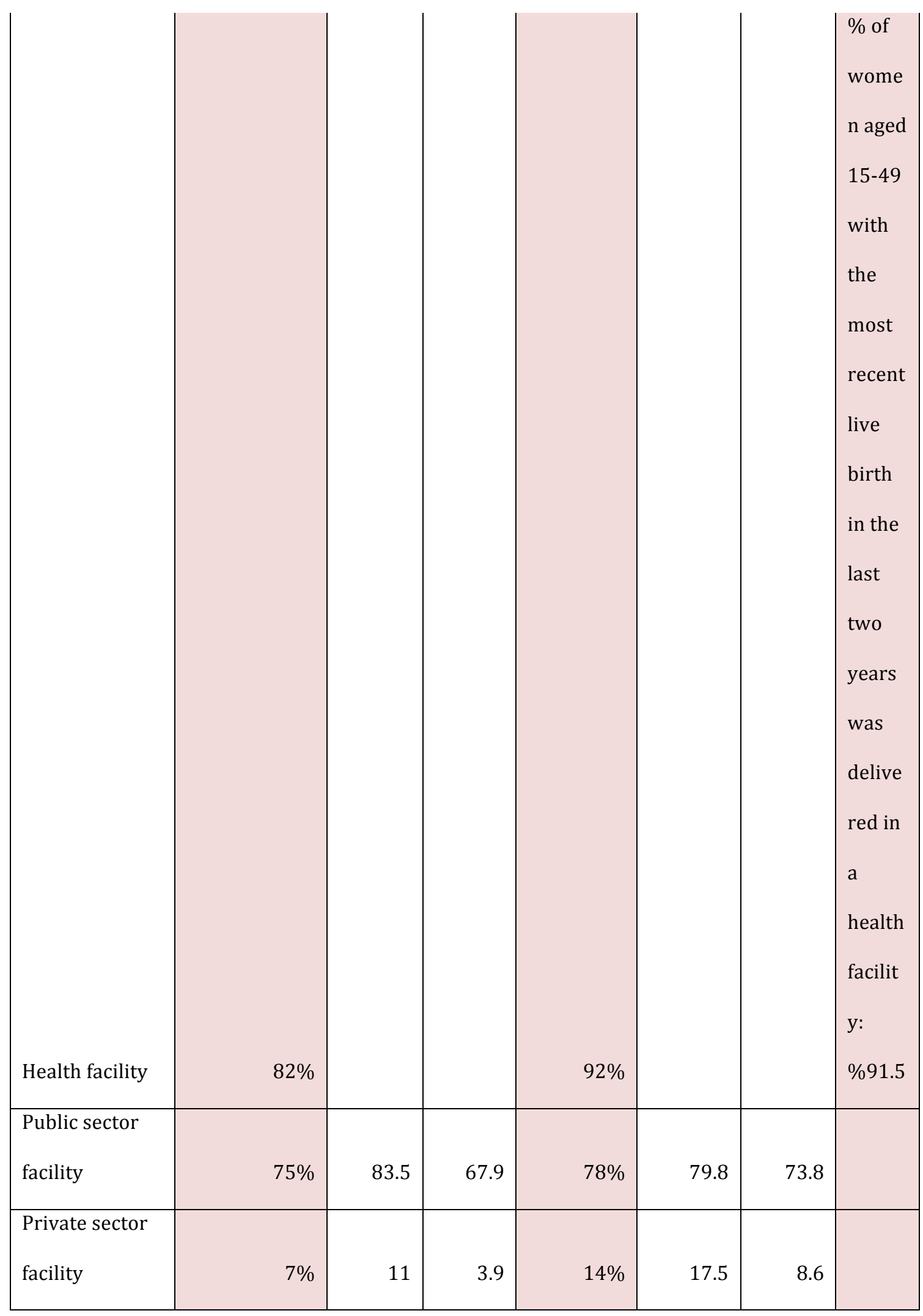




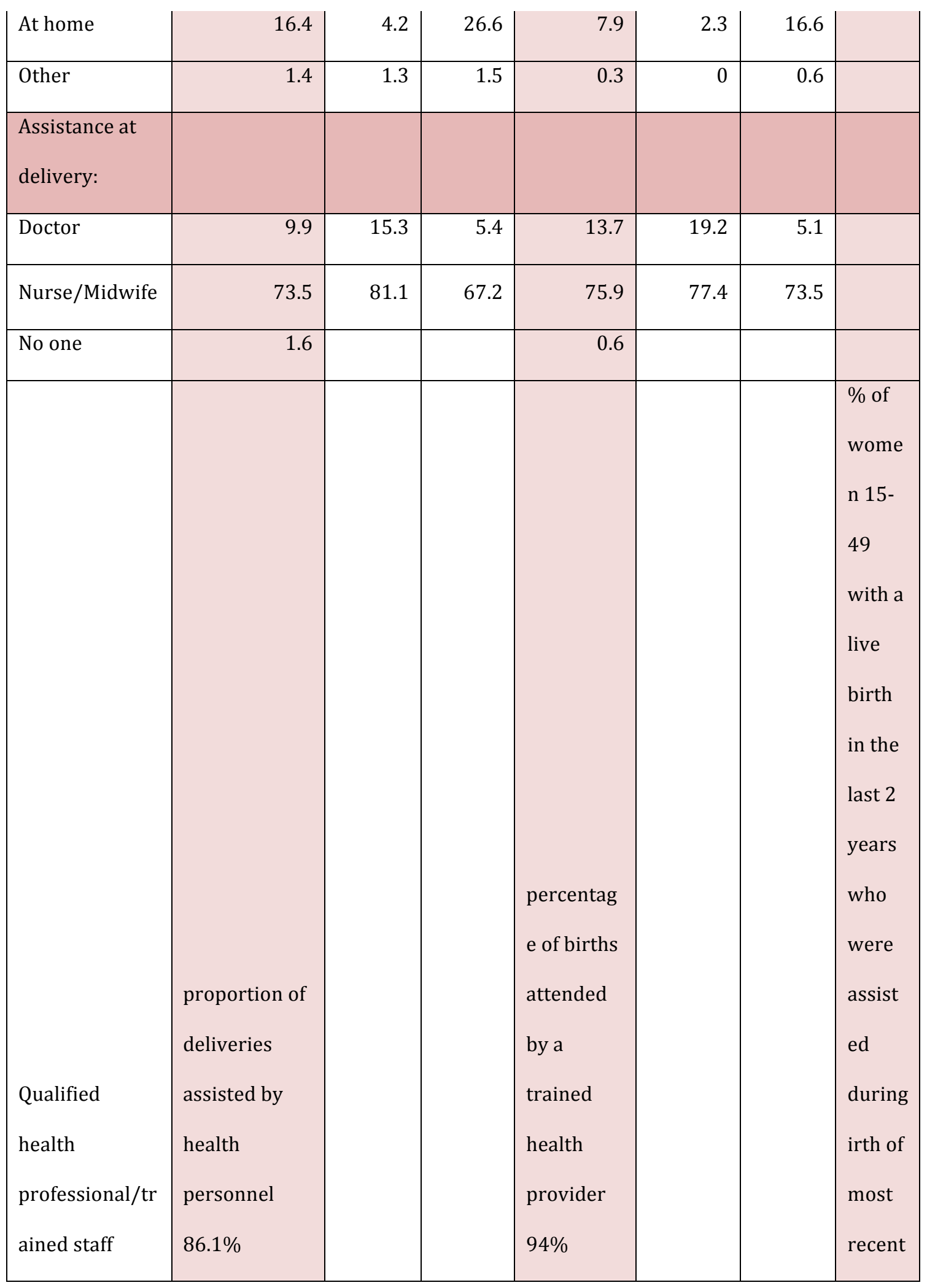




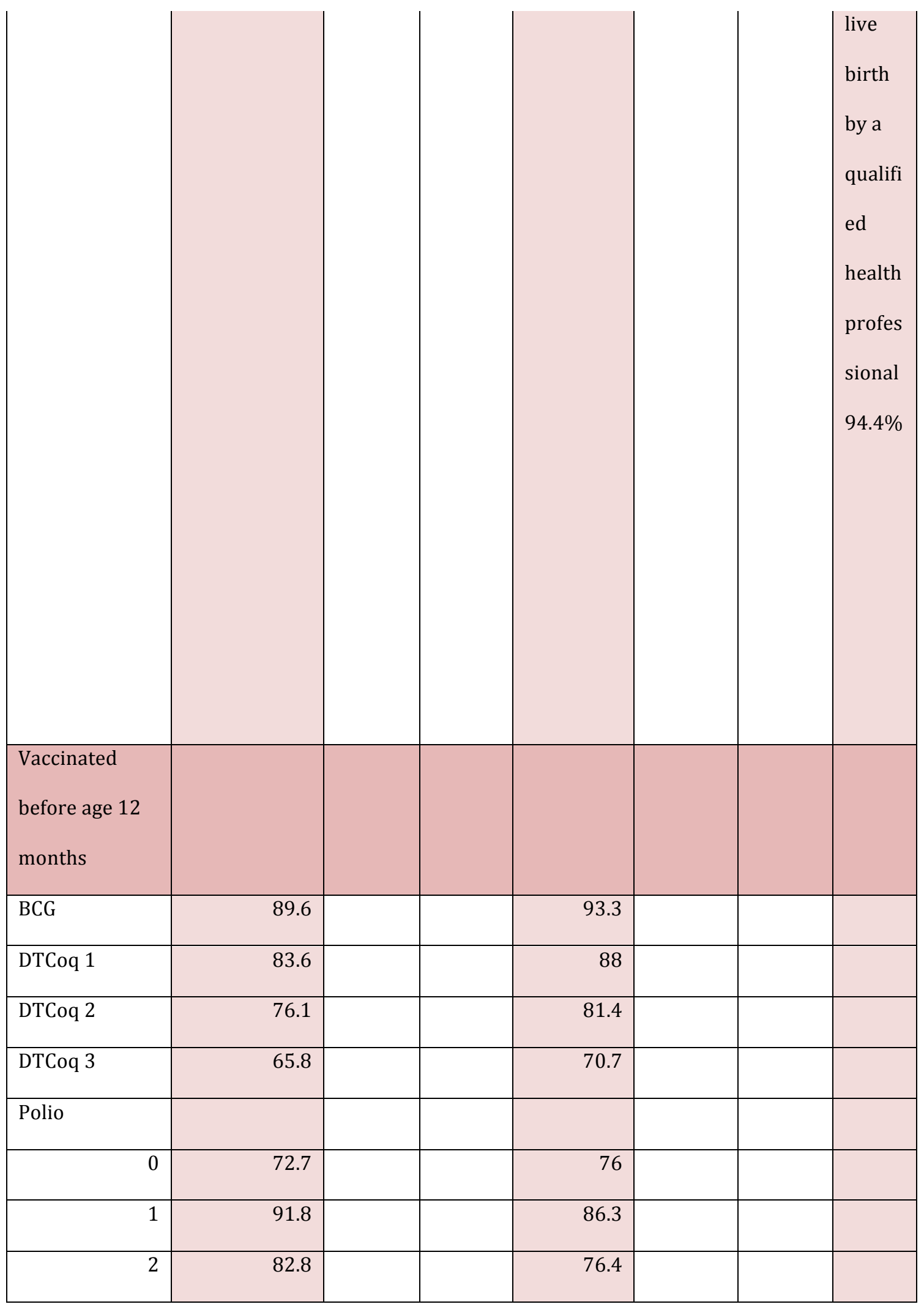




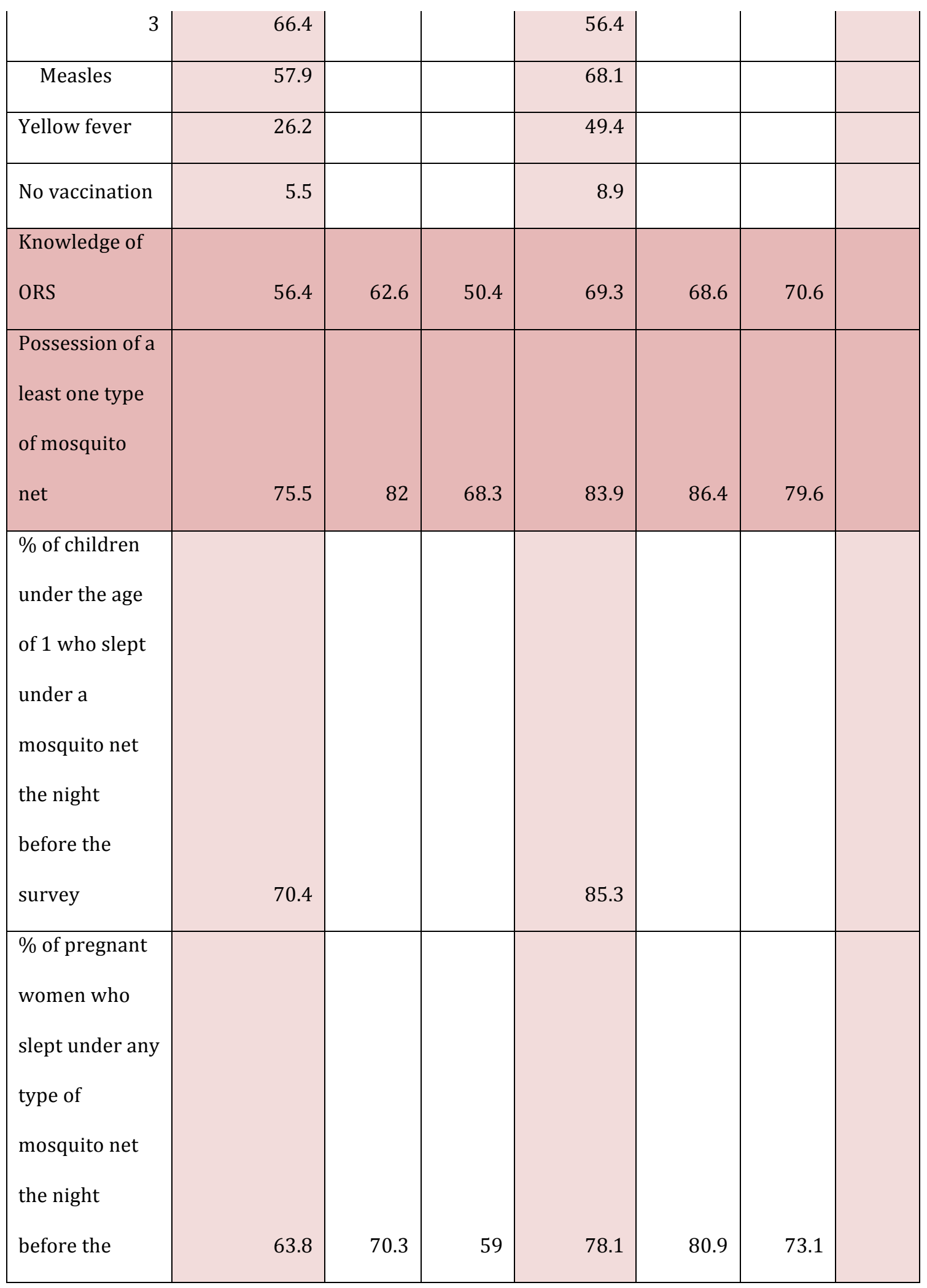




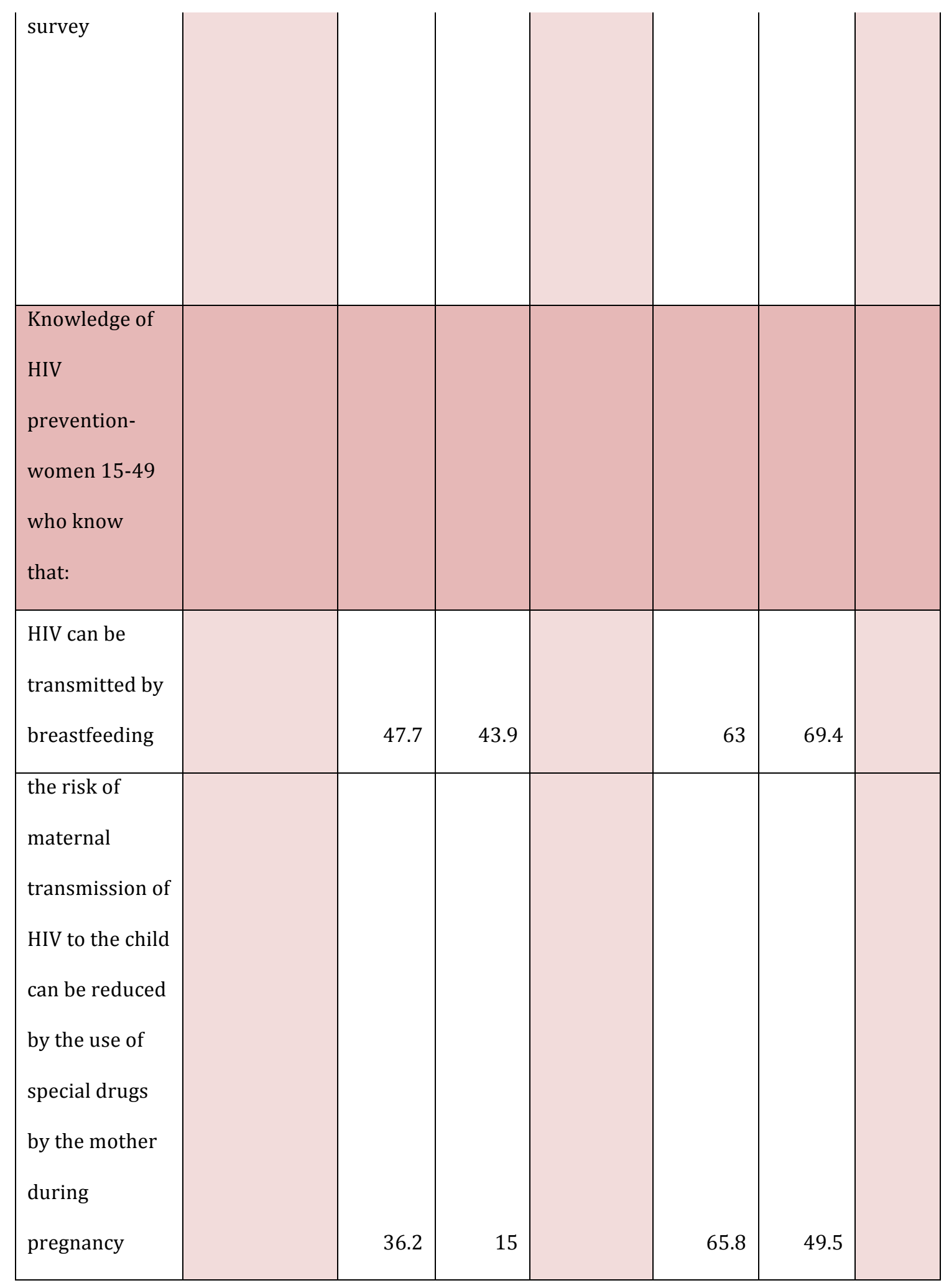


Appendix D: Republic of Yemen Data

The Demographic Health Survey (DHS) from 1997 and 2013 do not ask identical sets of questions; however, there are some similar questions and this case study will focus on some of the select data points to see how they potentially impact the IMR over the sixteen-year period. The areas of the surveys that had similar questions in both 1997 and 2013 are proportion of ever married women who saw a family planning message on television, median age at first marriage among women age 20 to 49 , age of mother at birth for neonatal mortality, age of mother at birth for post neonatal mortality, age of mother at birth for infant morality, birth intervals, age at first birth, contraceptive prevalence rate among married women, IMR based on mother's age at birth, IMR by birth order, ever married women: knowledge of family planning methods and sources, mean ideal number of children for ever married women by age, infant and child mortality levels, source of antenatal care, number of antenatal care visits, tetanus toxoid vaccinations and supplements, antenatal care provider, place of delivery, assistance at delivery. These different data points are organized into three categories for discussion: preconception, antenatal care, and delivery. 
Appendix E: Select Survey Data for Republic of Yemen

Republic of Yemen: Select comparison of survey data from Demographic Health Surveys (DHS) 1997, and (DHS) 2013.

\begin{tabular}{|c|c|c|c|c|c|c|}
\hline & $\begin{array}{c}\text { DHS } \\
\text { (1997) }\end{array}$ & $\begin{array}{c}\text { DHS Urban } \\
\text { (1997) }\end{array}$ & $\begin{array}{c}\text { DHS Rural } \\
\text { (1997) }\end{array}$ & $\begin{array}{c}\text { DHS } \\
(2013)\end{array}$ & $\begin{array}{c}\text { DHS } \\
\text { Urban } \\
\text { (2013) }\end{array}$ & $\begin{array}{c}\text { DHS Rural } \\
\text { (2013) }\end{array}$ \\
\hline $\begin{array}{l}\text { Housing } \\
\text { characteristics: }\end{array}$ & & & & & & \\
\hline Spring & 11.8 & 0.2 & 15.6 & 9.9 & 0.2 & 14.2 \\
\hline Tanker truck & 5.1 & 7.8 & 4.2 & 12.7 & 19.8 & 9.4 \\
\hline Bottled water & 3.7 & 14.6 & 0.1 & 11.5 & 34.7 & 1 \\
\hline $\begin{array}{l}\text { The proportion } \\
\text { of ever married } \\
\text { women who } \\
\text { saw a family } \\
\text { planning } \\
\text { message on } \\
\text { television }\end{array}$ & 22 & & & 46 & & \\
\hline $\begin{array}{l}\text { Median age at } \\
\text { first marriage } \\
\text { among women } \\
\text { age } 20 \text { to } 49\end{array}$ & & 16.9 & 16.3 & & 19.6 & 18.3 \\
\hline $\begin{array}{l}\text { Age of mother } \\
\text { at birth for } \\
\text { neonatal }\end{array}$ & & & & & & \\
\hline
\end{tabular}




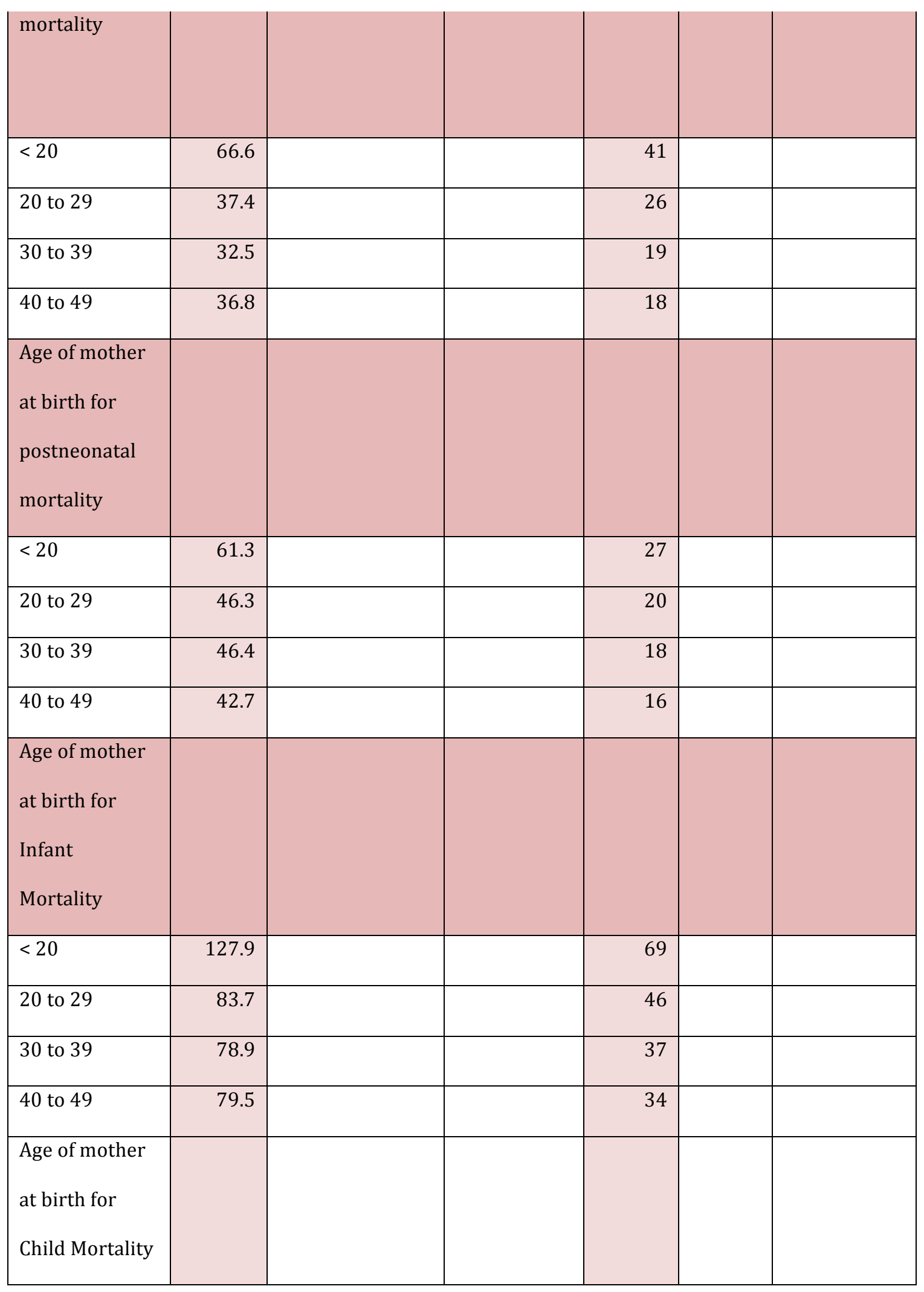




\begin{tabular}{|c|c|c|c|c|c|c|}
\hline$<20$ & 38.4 & & & 12 & & \\
\hline 20 to 29 & 32.6 & & & 11 & & \\
\hline 30 to 39 & 35.5 & & & 11 & & \\
\hline Birth intervals: & & & & & & \\
\hline 7 months -17 & & & & & & \\
\hline months & & 18.5 & 19.5 & & 11.8 & 14.8 \\
\hline $18-23$ & & 16.5 & 17.5 & & 13.5 & 16.8 \\
\hline $24-35$ & & 30.7 & 32.1 & & 25.3 & 30.3 \\
\hline $36-47$ & & 15.2 & 17.3 & & 18.3 & 17.4 \\
\hline $48+$ & & 19.1 & 13.6 & & 31.1 & 20.6 \\
\hline $\begin{array}{l}<2 \text { years } \\
\text { preceding birth } \\
\text { interval for } \\
\text { IMR compared } \\
\text { withbirth } \\
\text { spacing } 2 \text { years } \\
\text { or more }\end{array}$ & $\begin{array}{l}124.2 \\
\text { deaths } \\
\text { per } \\
1,000 \\
\text { compar } \\
\text { ed to } \\
36-55 \\
\text { deaths } \\
\text { per } \\
1,000\end{array}$ & & & $\begin{array}{l}73 \\
\text { deaths } \\
\text { per } \\
1,000 \\
\text { compar } \\
\text { ed to } \\
17-35 \\
\text { deaths } \\
\text { per } \\
1,000 \text { if } \\
\text { waiting } \\
2 \text { or } 3 \\
\text { years } \\
\text { birth } \\
\text { spacing }\end{array}$ & & \\
\hline
\end{tabular}




\begin{tabular}{|c|c|c|c|c|c|c|}
\hline $\begin{array}{l}\text { Age at first } \\
\text { birth: }\end{array}$ & & & & & & \\
\hline $\begin{array}{l}\text { Median age at } \\
\text { first birth for } \\
\text { women age 25- } \\
49\end{array}$ & & 19.3 & 19.5 & & 21.1 & 20.7 \\
\hline $\begin{array}{l}\text { Contraceptive } \\
\text { prevalence rate } \\
\text { among married } \\
\text { women }\end{array}$ & 21 & & & 34 & & \\
\hline $\begin{array}{l}\text { IMR based on } \\
\text { Mother's age at } \\
\text { birth }\end{array}$ & & & & & & \\
\hline$<20$ & 127.9 & & & 69 & & \\
\hline 20 to 29 & 83.7 & & & 46 & & \\
\hline 30 to 39 & 78.9 & & & 37 & & \\
\hline 40 to 49 & 79.5 & & & 34 & & \\
\hline $\begin{array}{l}\text { IMR by birth } \\
\text { order }\end{array}$ & & & & & & \\
\hline 1 & 110.2 & & & 59 & & \\
\hline 2 to 3 & 90.4 & & & 49 & & \\
\hline 4 to 6 & 76.1 & & & 39 & & \\
\hline $7+$ & 92.5 & & & 44 & & \\
\hline
\end{tabular}




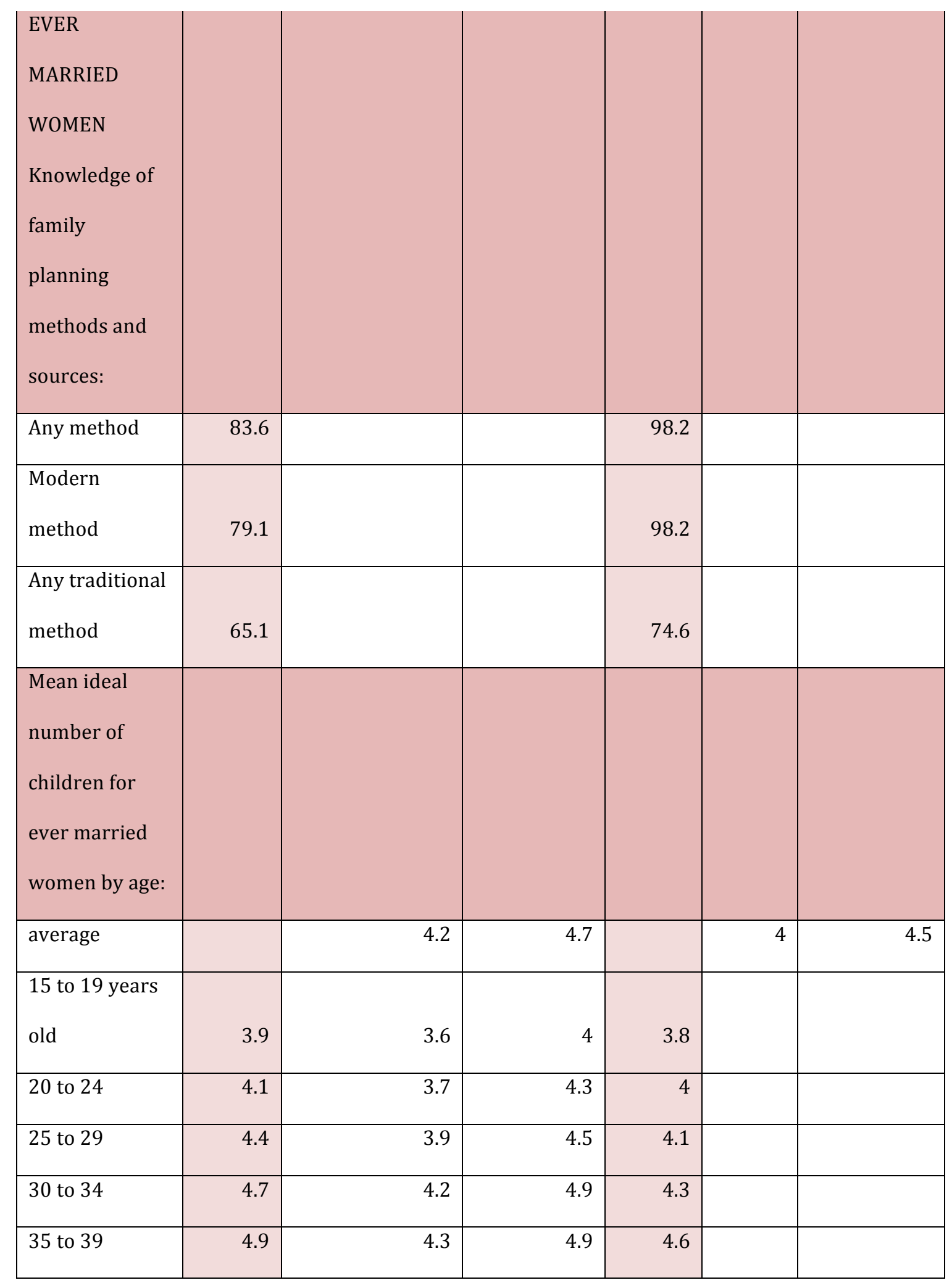




\begin{tabular}{|c|c|c|c|c|c|c|}
\hline 40 to 44 & 5.3 & 4.6 & 5.5 & 4.9 & & \\
\hline 45 to 49 & 5 & 5.2 & 5 & 5.2 & & \\
\hline $\begin{array}{l}\text { Infant and } \\
\text { child mortality } \\
\text { levels: }\end{array}$ & & & & & & \\
\hline $\begin{array}{l}\text { Neonatal } \\
\text { mortality }\end{array}$ & 40.8 & 38.4 & 41.5 & & 21 & 28 \\
\hline $\begin{array}{l}\text { Postneonatal } \\
\text { mortality }\end{array}$ & 48.7 & 37 & 52.1 & & 15 & 22 \\
\hline $\begin{array}{l}\text { Infant } \\
\text { mortality }\end{array}$ & 89.5 & $\begin{array}{l}75 \text { deaths per } \\
1,000 \text { live births }\end{array}$ & $\begin{array}{l}94 \text { deaths } \\
\text { per } 1,000 \\
\text { live births }\end{array}$ & $\begin{array}{l}43 \\
\text { deaths } \\
\text { per } \\
1,000 \\
\text { live } \\
\text { births }\end{array}$ & 36 & 51 \\
\hline Child mortality & & & & & 8 & 12 \\
\hline $\begin{array}{l}\text { Source of } \\
\text { Antenatal Care } \\
\text { (ANC): }\end{array}$ & & & & & & \\
\hline Doctor & 31.4 & & & 55.2 & & \\
\hline $\begin{array}{l}\text { Trained } \\
\text { nurse/midwife }\end{array}$ & 2.9 & & & 4.4 & & \\
\hline $\begin{array}{l}\text { Traditional } \\
\text { birth attendant }\end{array}$ & 0.6 & & & 0.2 & & \\
\hline No one & 65 & & & 38.4 & & \\
\hline
\end{tabular}




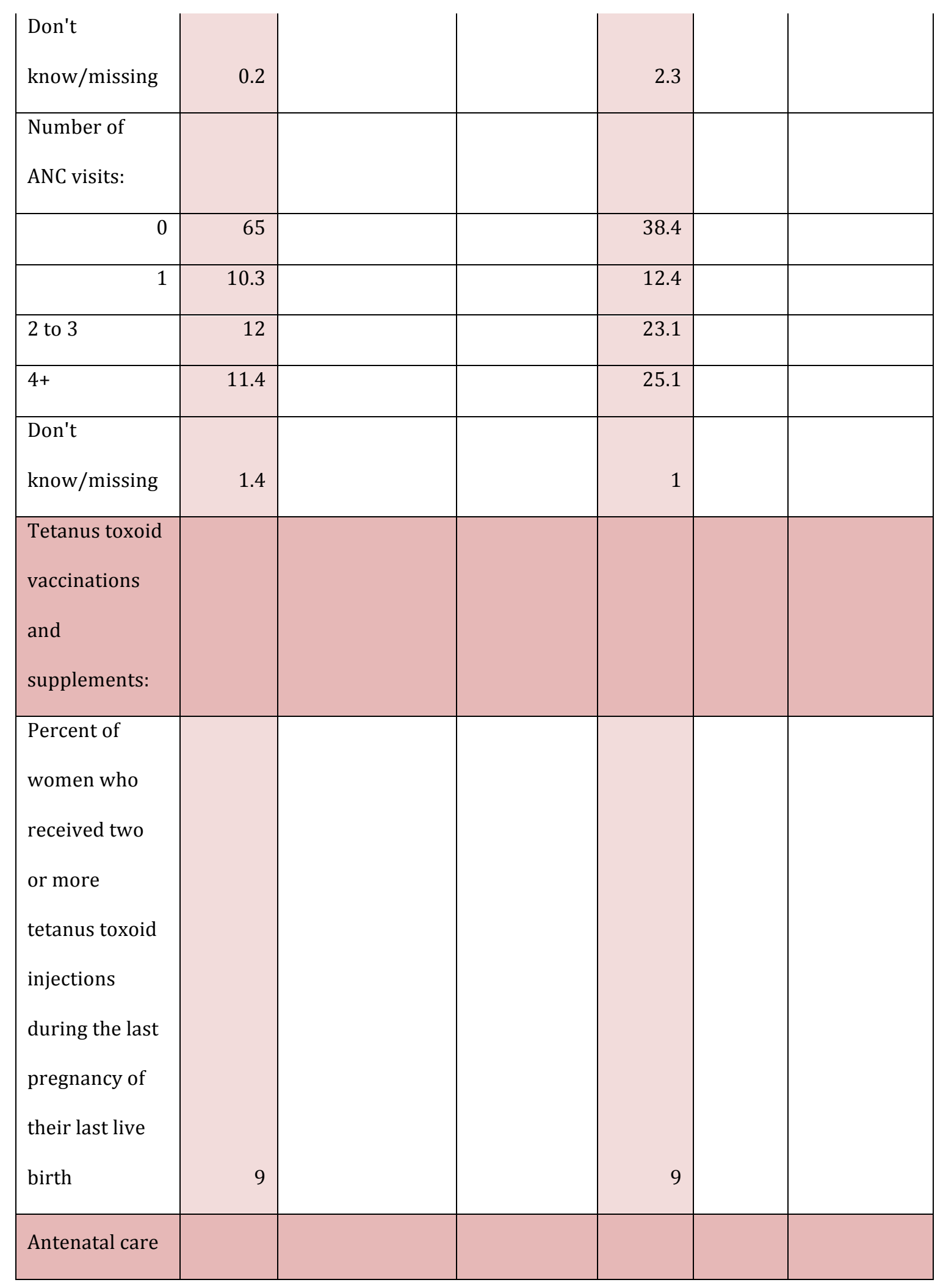




\begin{tabular}{|c|c|c|c|c|c|c|}
\hline provider: & & & & & & \\
\hline $\begin{array}{l}\text { Women } \\
\text { receiving ANC } \\
\text { from trained } \\
\text { medical } \\
\text { personnel }\end{array}$ & 34 & & & 60 & & \\
\hline Doctor & & 56 & 24.2 & & 75.1 & 46.8 \\
\hline $\begin{array}{l}\text { Nurse/Trained } \\
\text { midwife }\end{array}$ & & 5 & 2.3 & & 4.8 & 4.5 \\
\hline $\begin{array}{l}\text { Traditional } \\
\text { birth attendant }\end{array}$ & & 0.3 & 0.7 & & 0.1 & 0.2 \\
\hline None & & 38.5 & 72.7 & & 18.8 & 46.6 \\
\hline $\begin{array}{l}\text { Place of } \\
\text { delivery: }\end{array}$ & & & & & & \\
\hline Health facility & 16 & 30.6 & 11.1 & 29.8 & & \\
\hline $\begin{array}{l}\text { Public sector } \\
\text { facility }\end{array}$ & & & & & & \\
\hline $\begin{array}{l}\text { Private sector } \\
\text { facility }\end{array}$ & & & & & & \\
\hline At home & 84 & 69.1 & 88.6 & 68.5 & & \\
\hline Other & & 0 & & 0.6 & & \\
\hline Missing & & 0.3 & 0.2 & 1.1 & & \\
\hline $\begin{array}{l}\text { Assistance at } \\
\text { delivery: }\end{array}$ & & & & & & \\
\hline Doctor & 15 & 28.8 & 11 & 25.5 & & \\
\hline
\end{tabular}




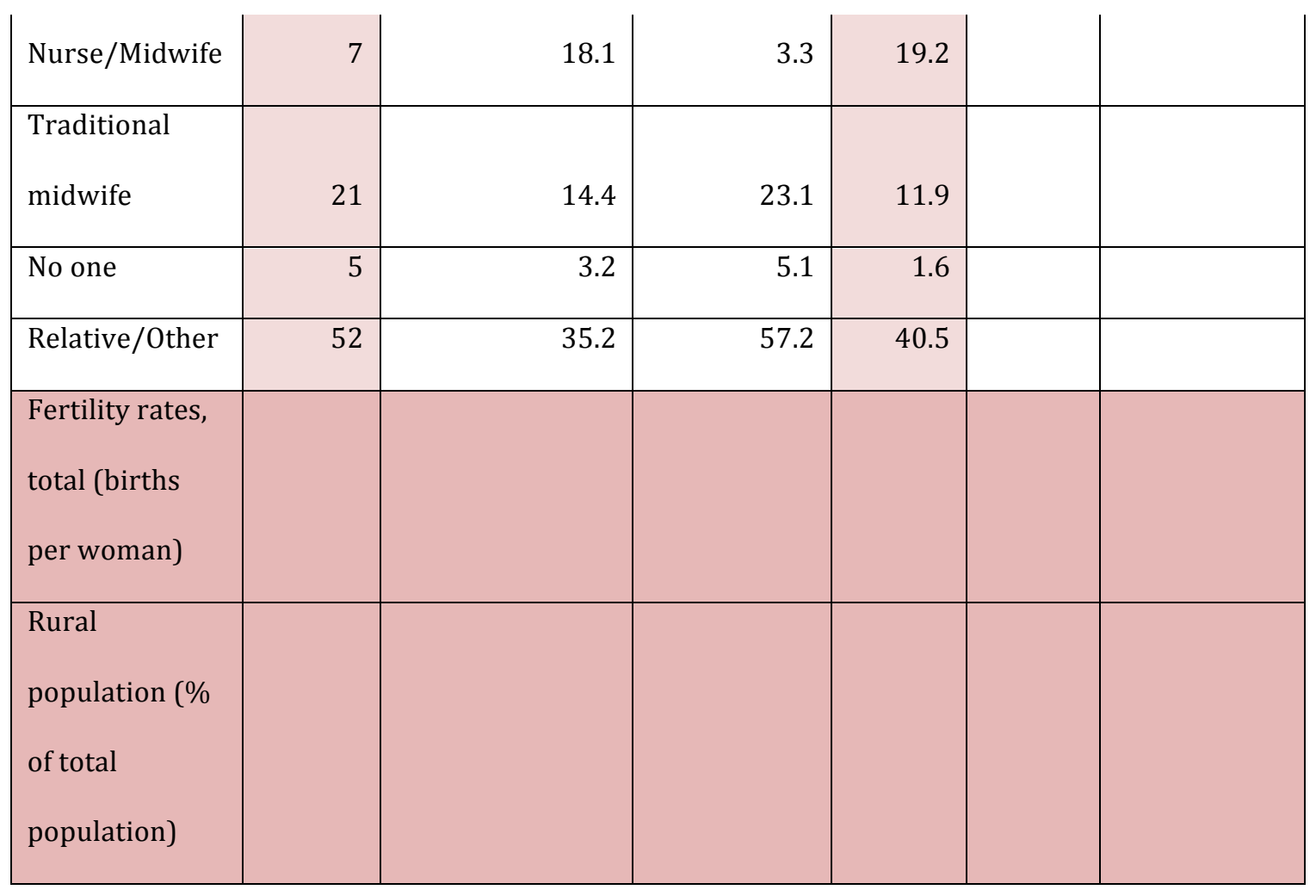

